\title{
Kleinskalige Magnetfelder der Sonne und ihr Einfluß auf Chromosphäre, Übergangszone und Korona
}

\author{
Dissertation \\ zur Erlangung des Doktorgrades \\ der Mathematisch-Naturwissenschaftlichen Fakultäten \\ der Georg-August-Universität zu Göttingen
}

vorgelegt von

Volker Wilken

aus Northeim

Göttingen 2001 
D 7

Referent:

Prof. Dr. F. Kneer

Korreferent:

Prof. Dr. E. Marsch

Tag der mündlichen Prüfung: 28.6.2001 


\section{Inhaltsverzeichnis}

$\begin{array}{lll}1 & \text { Einleitung } & 1\end{array}$

2 Die Sonne 3

$\begin{array}{lll}3 & \text { Theoretische Grundlagen } & 9\end{array}$

3.1 Stokes-Vektor . . . . . . . . . . . . . . . . . . . . . . . . . . . . . . . 9 9

3.2 Nachweis von Magnetfeldern . . . . . . . . . . . . . . . . . . . . . . 11

3.3 Modellrechnungen im Rahmen der Potentialfeldnäherung . . . . . . . . . 13

$\begin{array}{lll}4 & \text { Instrumentarium } & 18\end{array}$

4.1 Gregory-Coudé-Teleskop . . . . . . . . . . . . . . . . . . . . . . . . . . . 18

4.1 .1 Optischer Aufbau . . . . . . . . . . . . . . . . . . . . . . . . . . . 19

4.2 Vakuum-Turm-Teleskop . . . . . . . . . . . . . . . . . . . . . 20

4.3 Solar Ultraviolet Measurements of Emitted Radiation . . . . . . . . . . . 21

4.3 .1 Optischer Aufbau . . . . . . . . . . . . . . . . . . . 22

5 Beobachtungen und Datenreduktion $\quad 24$

5.1 Beobachtungskampagne . . . . . . . . . . . . . . . . 24

5.2 Daten vom Gregory-Coudé-Teleskop . . . . . . . . . . . . . . . . . . . . . 24

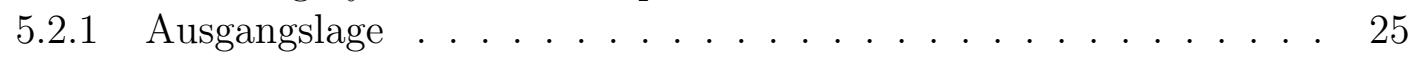

5.2 .2 Datenverarbeitung . . . . . . . . . . . . . . . . 25

$5.2 .3 \quad$ Physikalische Auswertung . . . . . . . . . . . . . . . . . . . . . 29

5.3 Daten vom Vakuum-Turm-Teleskop . . . . . . . . . . . . . . . . . . . 35

5.3 .1 Datenbearbeitung . . . . . . . . . . . . . . . . . 35

5.4 Daten von SUMER . . . . . . . . . . . . . . . . . . . . . . . . . . . . . . 36

5.4 .1 Datenbearbeitung . . . . . . . . . . . . . . . . . . . . . . . . . . . . . . 37

5.4 .2 Bestimmung physikalischer Größen . . . . . . . . . . . . . . . . . 37

6 Koordination der Beobachtung $\quad 41$

7 Auswertung der Übersichtsbilder 44

8 Die GCT-Bildserie 53 
9 Ergebnisse der Modellrechnungen $\quad 65$

\begin{tabular}{ll}
\hline 0 Die $x t$-Diagramme & 82
\end{tabular}

\begin{tabular}{ll}
\hline 1 Analyse der SUMER-Spektren & 95
\end{tabular}

11.1 Bereich 1. . . . . . . . . . . . . . . . . . . . . 995

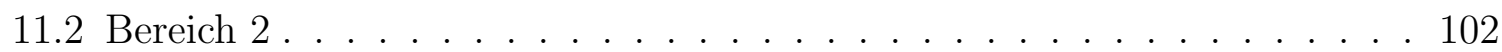

11.3 Bereich $3 . \ldots \ldots \ldots \ldots$

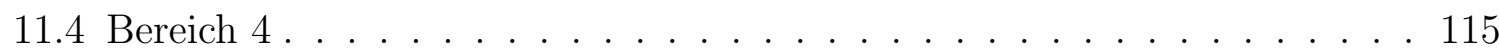

11.5 Bereich $5 . \ldots \ldots \ldots \ldots \ldots$

11.6 Bereich 6 . . . . . . . . . . . . . . . . . . . . . . . . . . . . . . . . 125

11.7 Bereich $7 \ldots \ldots \ldots \ldots \ldots$

11.8 Bereich 8 . . . . . . . . . . . . . . . . . . . . . . . . . . . . . . . 135

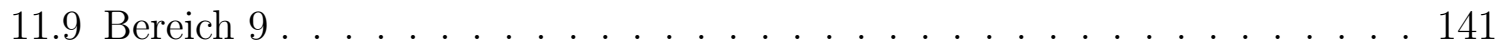

12 Zusammenfassung $\quad 146$

\begin{tabular}{ll}
\hline Literaturverzeichnis & 151
\end{tabular}

$\begin{array}{ll}\text { Dank } & 157\end{array}$

\begin{tabular}{ll}
\hline Lebenslauf & 158
\end{tabular} 


\section{Einleitung}

Obwohl die Sonne ein gewaltiger, zu ca. $73 \%$ aus Wasserstoff und ca. 25\% aus Helium bestehender Gasball mit einer Masse von 1,99 $\cdot 10^{30} \mathrm{~kg}$ ist, was ungefähr $99,86 \%$ der Gesamtmasse des Sonnensystems entspricht, nimmt sie sich als einer von einigen 100 Milliarden Sternen unserer Galaxis, die ja auch wieder nur eine von ca. 50 Milliarden Galaxien des sichtbaren Universums ist, als verhältnismäßig kleiner Hauptreihenstern vom Spektraltyp G2V doch sehr durchschnittlich aus. So ist also auch der für uns fast unvorstellbare Sonnendurchmesser von etwa 1,4 Millionen km, was 109 Erddurchmessern entspricht und von der Erde aus unter einem Winkel von ungefähr einem halben Grad erscheint, aus astrophysikalischer Sicht wenig eindrucksvoll. Daß der Mond von der Erde aus gesehen unter demselben Winkel erscheint und somit bei totalen Sonnenfinsternissen die Entdeckung der solaren Chromospäre und Korona ermöglichte, ist jedoch wahrlich eine sonderbare Laune der Natur.

Gegenstand der vorliegenden Arbeit ist die Untersuchung kleinskaliger Magnetfelder der Sonne und deren Einfluß auf die darüberliegenden Atmosphärenschichten. Es sind gerade die vielen kleinen überall auf der Sonne vorkommenden solaren Magnetfelder und ihre Wechselwirkung mit dem solaren Gas, die für die Struktur und Dynamik der Sonnenatmosphäre von entscheidender Bedeutung sind. Die Magnetfelder der Sonne beschleunigen den Sonnenwind und nehmen so Einfluß auf die Heliosphäre, einschließlich dem Erdklima. Für die Untersuchung wurde ein Gebiet schwachen magnetischen Flusses in der Sonnenscheibenmitte ausgewählt und über ca. drei Stunden hinweg mit drei Teleskopen in verschiedenen Spektralbereichen beobachtet. Mit der Absorptionslinie des neutralen Eisens bei 630,25 nm wurde die Entwicklung des Objektes in der Photosphäre festgehalten. Weiterhin wurden Filtergramme in der Ca $K$-Linie aufgenommen, die ganz ausgezeichnet die Verhältnisse der unteren Chromosphäre wiedergeben. Gleichzeitig wurden Aufnahmen in den Emissionslinien des neutralen Kohlenstoffs (C I 156,10 nm), des dreifach ionisierten Kohlenstoffs (C IV 154,82 nm) und des siebenfach ionisierten Neons (Ne VIII 77,04 nm) gemacht, um die Geschehnisse in der Chromosphäre, Übergangsregion und unteren Korona zu dokumentieren. Mit dieser zeitlichen und räumlichen Abdeckung des ausgesuchten magnetischen Gebietes, eines Teils des chromosphärischen Netzwerkes, ist es gelungen, die mit diesem Gebiet verbundenen hoch dynamischen Prozesse zu erfassen und zu beschreiben.

Der Arbeit liegt folgende Gliederung zugrunde:

Zunächst wird in Kapitel 2 eine kurze Zusammenfassung der Verhältnisse und Er- 
scheinungen in bzw. auf der Sonne im Hinblick auf das Untersuchungsobjekt gegeben. Hierzu wurden die Lehrbücher von Glassmeier/Scholer, Scheffler/Elsässer, Stix, Voigt, Unsöld/Baschek, Unsöld und Mihalas zu Rate gezogen. Im Kapitel 3 werden die theoretischen Beschreibungsweisen und Nachweismethoden zusammengefaßt. Die hier gemachten Aussagen stützen sich auf den Artikel von Landi Degl'Innocenti und die Bücher Haken/Wolf, Mayer-Kuckuk, Chandrasekhar, Shurcliff, Jackson, Priest und Kippenhahn/Möllenhoff. In Kapitel 4 werden anschließend die beteiligten Teleskope und deren optischer Aufbau skizziert. In den folgenden beiden Kapiteln 5 und 6 werden die Datenerhebung und -reduktion sowie die Probleme der Beobachtungskoordination besprochen. In Kapitel 7 werden dann die Übersichtsbilder gezeigt, die unter anderem Aufschluß über die Art des Beobachtungsobjektes geben. In Kapitel 8 wird die ganze Serie von Magnetogrammen gezeigt und Probleme der Kompensation instrumenteller Bildbewegung diskutiert. In Kapitel 9 werden dann die Ergebnisse von Potentialfeldextrapolationsrechnungen präsentiert. Anschließend werden in Kapitel 10 die aus der Beobachtung gewonnenen Datensätze in Form von $x t$-Diagrammen miteinander verglichen. Es folgt eine detaillierte Analyse der SUMER-Spektren in Kapitel 11. Schließlich wird in Kapitel 12 eine Zusammenfassung gegeben. 


\section{Die Sonne}

\section{Aufbau}

Im zentralen Bereich der Sonne mit einem Radius von ca. 0,3 Sonnenradien werden seit etwa 4,5 Milliarden Jahren bei einer Temperatur von $\sim 1,5 \cdot 10^{7} \mathrm{~K}$ und einer Dichte von $\sim 1,5 \cdot 10^{5} \mathrm{~kg} / \mathrm{m}^{3}$ pro Sekunde rund 400 Millionen Tonnen Wasserstoff zu Helium umgewandelt. So wird eine Leistung von $3,85 \cdot 10^{23} \mathrm{~kW}$ erzeugt. An diese nukleare Brennzone schließt sich bis knapp 3/4 des Sonnenradius der sogenannte radiative Kern an. Bei Temperaturen von $\sim 5,9 \cdot 10^{6} \mathrm{~K}$ bis $\sim 1,8 \cdot 10^{6} \mathrm{~K}$ (von innen nach außen) und Dichten von $\sim 7,5 \cdot 10^{3} \mathrm{~kg} / \mathrm{m}^{3}$ bis $\sim 1,1 \cdot 10^{2} \mathrm{~kg} / \mathrm{m}^{3}$ werden die durch Kernfusion im Inneren erzeugten Photonen nach wenigen Zentimetern wieder völlig absorbiert und reemittiert. Ein solches im Sonneninneren erzeugtes Quant benötigt einige Millionen Jahre, ehe es an der Oberfäche die Sonne verläßt. An diese Zone des Energietransports durch Strahlung schließt sich eine Zone des Energietransports durch Konvektion an, die sogenannte Wasserstoff-Konvektionszone. Hier haben nur 2\% der Sonnenmasse durch Konvektion und differentielle Rotation entscheidenden Anteil am Antrieb des Sonnendynamos, dessen induzierte Ströme die, auf der Sonne allgegenwärtigen, die äußeren Atmosphärenschichten dominierenden Magnetfelder mit Energie versorgen. An diese Zone, deren oberer Rand die Sonnenoberfläche bildet, schließt sich die $200 \mathrm{~km}$ bis $500 \mathrm{~km}$ dicke Photosphäre (Lichthülle) mit Temperaturen von ca. $5800 \mathrm{~K}$ und Dichten von $\sim 3 \cdot 10^{-5} \mathrm{~kg} / \mathrm{m}^{3}$ an. Ihr entstammt die, über $90 \%$ der Energieabgabe der Sonne enthaltende, Strahlung zwischen nahem Ultraviolett und fernem Infrarot. Die Sonne wird aufgrund der hier in den Weltraum emittierten Strahlung jede Sekunde um ca. 4 Millionen Tonnen "leichter". Nach einem Temperaturminimum von etwa $4100 \mathrm{~K}$ und Dichten von $\sim 5 \cdot 10^{-6} \mathrm{~kg} / \mathrm{m}^{3}$ schließt sich die Chromosphäre (Farbhülle) mit einer Ausdehnung von ca. $2000 \mathrm{~km}$ an. Sie endet an einer relativ schmalen Schicht, die durch ein massives Abfallen der Dichte und einen dramatischen Anstieg der Temperatur gekennzeichnet ist. Diese Schicht wird Übergangszone genannt. Bei Temperaturen von einigen $10^{6} \mathrm{~K}$ und Dichten von weniger als $5 \cdot 10^{-13} \mathrm{~kg} / \mathrm{m}^{3}$ erstreckt sich anschließend die Korona (Strahlenkranz) viele Sonnenradien weit in den Weltraum. Zusammen mit dem Temperaturanstieg erfährt das koronale Plasma eine Beschleunigung auf Überschallgeschwindigkeit. Dieses die Magnetfelder mitführende Plasma strömt dann als sogenannter schneller $(v>650 \mathrm{~km} / \mathrm{s})$ oder langsamer $(v<350 \mathrm{~km} / \mathrm{s})$ Sonnenwind kontinuierlich in den Raum und durchflutet und beeinflußt das gesamte Planetensystem. 


\section{Strahlung}

Die Sonne emittiert Strahlung über den weiten spektralen Bereich von der Röntgen- und Ultraviolettstrahlung über die sichtbare und infrarote Strahlung bis weit hinein in das Gebiet der Radiowellen. Das Sonnenspektrum entsteht in unterschiedlichen Höhen der Sonnenatmosphäre und stellt somit eine Überlagerung der Strahlung sehr verschiedener Schichten dar, mitunter auch mit Anteilen aus räumlich eng begrenzten Bereichen innerhalb einer Schicht.

Der wenige $100 \mathrm{~km}$ dicken, den optischen Sonnenrand definierenden Photosphärenschicht entstammt, wie schon erwähnt, über 90\% der Strahlung zwischen nahem Ultraviolett und fernem Infrarot. Obgleich sehr dünn, nehmen in dieser tiefsten Schicht, aus der wir überhaupt noch direkte Strahlung erhalten, die Temperatur und die Dichte beträchtlich mit der Tiefe zu, so daß sich Strahlungen unterschiedlich heißer Lagen superponieren. Bei Wellenlängen mit starker Linienabsorption kann die Strahlung nur aus den obersten kühlsten Photosphärenschichten kommen und ist deshalb schwächer als bei benachbarten Wellenlängen. Daher besteht das Sonnenspektrum vom nahen Ultraviolett $(\lambda \approx 200 \mathrm{~nm})$ bis zum fernen Infrarot $(\lambda \approx 1000000 \mathrm{~nm}=1 \mathrm{~mm})$ aus einem Kontinuum, in das dunkle Absorptionslinien, die sogenannten Fraunhoferlinien, eingelagert sind. Bei Wellenlängen deutlich kleiner $200 \mathrm{~nm}$ sind die Absorptionslinien verschwunden und dem mit der Wellenlänge massiv schwächer werdenden Kontinuum überlagern sich jetzt mehr und mehr die Emissionslinien, deren Ursprung in der Chromosphäre liegt. Oberhalb der Chromosphäre beginnt die Übergangsregion, eine Schicht, die, wie schon erwähnt, durch einen starken Temperaturanstieg definiert ist. Darüber entstehen in der weit ausgedehnten Korona drei weitere äußerst schwache Strahlungskomponenten. Zum einen sieht man ein schwaches Kontinuum (K-Korona), das vorwiegend aus Photosphärenlicht besteht, welches an den, wegen der hohen Temperatur von einigen Millionen Kelvin, zahlreichen freien Elektronen in der Korona gestreut wird. Weiter besteht die Strahlung aus Emissionslinien koronalen Ursprungs (E-Korona), die dieses Kontinuum überlagern. Und schließlich erkennt man weit außen das an Staub gestreute Photosphärenspektrum wieder (F-Korona). Die Korona erzeugt darüber hinaus die extrem kurzwelligen (Röntgenstrahlung) und extrem langwelligen (Radiostrahlung) Anteile des Sonnenspektrums.

\section{Erscheinungen und Strukturen}

In der Atmosphäre der Sonne spielen sich eine Vielzahl von Erscheinungen und Phänomenen ab. Man kann sie grob in zwei Bereiche unterteilen. Einerseits die sporadischen und periodischen Ereignisse, wie z.B. Flecken, Poren, Fackeln, Flares usw. . Sie werden als Erscheinungen der aktiven Sonne bezeichnet. Andererseits alle Phänomene, die sich während des gesamten 22-jährigen Sonnenzyklus beobachten lassen und überall auf 
der Sonne in Erscheinung treten. Man nennt sie Phänomene der ruhigen Sonne. Sie bilden die Kulisse, auf der sich die Ereignisse der aktiven Sonne abspielen, freilich nicht ohne von diesen zumeist massiv gestört zu werden. Beispiele dieser Hintergrundereignisse sind die Granulation, die Supergranulation, das chromosphärische Netzwerk und deren Intranetzwerk-Elemente usw. . Da im Zuge der vorliegenden Arbeit kleinskalige Magnetfelder beobachtet wurden, die dem Erscheinungsbild der ruhigen Sonne zuzurechnen sind, wird im folgenden nur noch von solchen Phänomenen die Rede sein.

Die durch die sehr dynamischen Vorgänge der Wasserstoff-Konvektionszone erzeugte Granulation bildet die photosphärische Feinstruktur. Ihr irreguläres, zellulares Muster besteht aus dicht aneinander liegenden hellen Granulen, die durch relativ schmale dunkle Kanäle voneinander getrennt sind. Die einzelne Granule ist ständiger Veränderung unterworfen und somit nach ca. 6 bis 12 Minuten nicht mehr wiederzuerkennen. Granulen haben typische Durchmesser von $500 \mathrm{~km}$ bis $1500 \mathrm{~km}$ (1" entspricht im Mittel $725 \mathrm{~km}$ auf der Sonne). Die Konvektionsgeschwindigkeit in der Photosphäre beträgt einige wenige Kilometer pro Sekunde. In ungestörten Bereichen weist sie eine magnetische Flußdichte von einigen Gauß (1 Gauß $[\mathrm{G}]=10^{-4}$ Tesla $[\mathrm{T}]$ ) auf. Die zunächst völlig irregulär erscheinende Struktur der Granulation organisiert sich zu größeren Gebilden, sogenannten Mesogranulen, von ca. 10" Durchmesser.

Monochromatische Bilder der ungestörten Chromosphäre weisen ein Muster feiner Strukturen auf. Diese Feinstruktur tritt besonders klar in $\mathrm{H} \alpha$-Filtergrammen auf. Man klassifiziert folgende Strukturelemente:

1.) fine mottles: kleine dunkle Körner mit Abmessungen von $600 \mathrm{~km}$ bis $1600 \mathrm{~km}$ und einer Lebensdauer von ca. 10 Minuten (engl. mottle = sprenkeln, marmorieren).

2.) coarse mottles: aus fine mottles gebildete rundliche Büschel mit Dimensionen von $2000 \mathrm{~km}$ bis $8000 \mathrm{~km}$ und Lebensdauern von einigen Stunden.

3.) Netzwerk: aus dunklen mottles gebildetes mehr oder weniger unregelmäßiges Netzwerk mit Maschenweiten von etwa $20000 \mathrm{~km}$ bis $40000 \mathrm{~km}$ und Lebensdauern von etwa einem Tag.

Das auf Filtergrammen des Ca II $K$-Linienkerns hervortretende helle Netzwerk fällt mit dem dunklen in $\mathrm{H} \alpha$ sichtbaren Netzwerk zusammen, obgleich die Feinstruktur unterschiedlich ist. Im Linienzentrum von $\mathrm{H} \alpha$ verschwindet das dunkle fine mottling, und es erscheint eine relativ dichte Besetzung mit kurzen fadenförmigen Gebilden (fibrils). Die fine mottles treten am Sonnenrand als Spiculen (engl. spicule = Nadel, nadelartiger Fortsatz) in Erscheinung. Die Maschen des chromosphärischen Netzwerkes sind identisch mit den Zellen der auf Dopplergrammen deutlich hervortretenden Supergranulation. Dieses von Leighton et al. (1962) mit Hilfe von Geschwindigkeitsheliogrammen gefundene großräumige zellulare Muster hat eine mittlere Maschengröße von ca. $30000 \mathrm{~km}$. Es zeichnet sich durch eine vorherrschende horizontale Bewegung aus. Materie steigt im Zellzentrum mit ca. $0,05 \mathrm{~km} / \mathrm{s}$ auf und strömt mit etwa $0,4 \mathrm{~km} / \mathrm{s}$ zum Zellenrand, um dort wieder mit ca. $0,1 \mathrm{~km} / \mathrm{s}$ abzusinken; eine konvektive Bewegung also. Anders als bei der Granulation scheinen die supergranularen Zellränder jedoch unverbundene isolierte Gebiete der Abwärtsströmung zu haben, die häufig mit Gebieten zusammenfallen, an 
denen sich einige Zellen berühren. Wie jedes große Geschwindigkeitsfeld auf einem rotierenden System, ist auch die Supergranulation der Corioliskraft unterworfen. Als Folge dessen verläuft die Materieströmung vom Zellenzentrum zum Rand leicht wirbelartig. Wie das chromosphärische Netzwerk, zerfallen auch die Supergranulen nach größenordnungsmäßig einem Tag.

\section{Kleinskalige Magnetfelder der ruhigen Sonne}

Die Magnetfelder der Sonne entstehen im Sonneninneren durch einen Dynamoprozeß. Verursacht durch magnetischen Auftrieb und konvektive Kräfte, treten sie an verschiedenen Stellen mit unterschiedlich starkem Fluß am Boden der Photosphäre aus, bleiben aber im Sonneninneren verankert. Die meisten Vorgänge in hohen Atmosphärenschichten der Sonne, wie Aufhellungen und schnelle Bewegungen, haben ihre Ursache im Verhalten der Magnetfelder in der Photosphäre und ihrer Wechselwirkung mit photoshärischem und subphotosphärischem Gas.

Das Verhalten eines zunächst homogenen Magnetfeldes unter Einfluß der schon beschriebenen zellularen Strömung in einer Supergranule wurde von Parker (1963), Weiss (1964), Clark und Johnson (1967), Galloway und Weiss (1981) und Galloway und Proctor (1983) modellhaft untersucht. Es konnte gezeigt werden, daß sich fast der gesamte magnetische Fluß nach einiger Zeit an den Zellrändern und Zellecken sammelt. Beobachtungen bestätigen diesen Sachverhalt im wesentlichen. Wie im Modell, neigen die Magnetfelder dazu, sich an den Rändern und besonders in den Ecken zu konzentrieren; jedoch konnten auch Strukturen geringen magnetischen Flusses, sogenannte Intra-Netzwerk-Strukturen, nachgewiesen werden (Livingston, Harvey 1975 und Smithson 1975). Diese punktartigen Elemente verschiedener, nicht immer ausgeglichener Polarität bewegen sich nicht immer radial mit $0,1 \mathrm{~km} / \mathrm{s}$ bis $0,3 \mathrm{~km} / \mathrm{s}$ auf die Ränder der Netzwerkzellen zu (Wang et al. 1995). 1996 beobachteten Wang et al., daß sich die Intra-Netzwerk-Strukturen zunächst mit ca. $0,25 \mathrm{~km} / \mathrm{s}$ bis $0,4 \mathrm{~km} / \mathrm{s}$ vom Zentrum der Zelle zu deren Rand bewegen und dort dann entlang der Netzwerkränder mit $0,05 \mathrm{~km} / \mathrm{s}$ auf die Netzwerkecken zu. Neben diesen Intra-Netzwerk-Elementen, deren Magnetfeld mehr oder weniger senkrecht aus der Sonnenoberfläche austritt, wurde von Martin 1988 und später von Lites et al. 1996 auch eine Horizontalkomponente des Intra-Netzwerk-Feldes beobachtet. Weiterhin konnte durch Beobachtungen von Stenflo 1982 und Bianda et al. 1998 eine schwache, turbulente, nicht aufgelöste Magnetfeldkomponente nachgewiesen werden. Der Vergleich von Magnetogrammen des im Zuge der Arbeit ausgewerteten Datensatzes mit Ca-Filtergrammen desselben Tages zeigt deutlich, daß hier Netzwerkelemente die Objekte der intensiven Betrachtung waren. Wang 1988 schließt von quantitativen Beobachtungen mit einem Videomagnetographen auf folgende Eigenschaften dieser Elemente:

1.) In der ruhigen Sonne variiert der mittlere magnetische Gesamtfluß über 10 Stunden nicht. Das Verschwinden und Erscheinen des Flusses ist also ausgeglichen.

2.) Die magnetischen Flußröhren des Netzwerkes haben eine typische Größe von $5^{\prime \prime}$ bis $7^{\prime \prime}$. 
3.) Die mittlere horizontale Geschwindigkeit der magnetischen Netzwerkelemente ist $\leq 0,14 \mathrm{~km} / \mathrm{s}$.

4.) Die effektive magnetische Diffusionskonstante für ruhige Gebiete gemischter Polarität ist $\leq 150 \mathrm{~km}^{2} / \mathrm{s}$. In "enhanced network" Gebieten (engl. enhance = erhöhen, vergrößern, steigern) ist die Diffusionskonstante $<100 \mathrm{~km}^{2} / \mathrm{s}$.

In den letzten 50 Jahren wurde die Bedeutung kleinskaliger Magnetfelder für die Struktur und Dynamik der Sonnenatmosphäre, auch der ruhigen, nicht aktiven Sonnenatmosphäre, immer deutlicher. Seit dem Start der Raumsonde SOHO (Solar and Heliospheric Observatory) im Dezember 1995 mit seinen zwölf wissenschaftlichen Instrumenten an Bord, steht der Sonnenforschung eine phantastische Breite zusätzlicher Beobachtungsmöglichkeiten zur Verfügung. Es lassen sich z.B., wie im Rahmen der vorliegenden Arbeit geschehen, simultane Beobachtungen mit dem EUV-Teleskop SUMER (Solar Ultraviolet Measurements of Emitted Radiation) auf SOHO und den bodengebundenen Sonnenteleskopen am Observatorio del Teide auf Teneriffa realisieren. Auf diese Weise ist es möglich, in verschiedenen Wellenlängenbereichen mit geeigneten Spektrallinien, deren Licht aus verschiedenen Höhen der Sonnenatmosphäre emittiert wird, Objekte der Sonne zu studieren.

Innes et al. 1997 untersuchten mit SUMER in der Si IV (139,3 nm) Emissionslinie des ultravioletten Spektralbereichs explosive Ereignisse in dem supergranularen Netzwerk der ruhigen Sonne. Es war schon seit längerem bekannt, daß die Ränder und Knotenpunkte des supergranularen Musters, an denen sich, wie schon beschrieben, magnetischer Fluß konzentriert, ein steter Quell veränderlicher und immer wieder extrem heftiger Aktivität ist. Plötzlich auftauchende starke, kleinskalige Plasmaströmungen (engl. plasma jets), sehr kleine aufflackernde Lichter (engl. micro flares; Porter et al. 1987, Rabin und Dowdy 1992) und explosive Ereignisse (engl. explosive events; Brueckner und Bartoe 1983, Dere et al. 1989, Dere 1994) sind Beispiele für diese Aktivität. Daß explosive Ereignisse in Salven (engl. bursts) von bis zu 30 Minuten erscheinen, was von Dere 1994 vorhergesagt wurde, können Innes et al. 1997 bestätigen. Einzelne Ereignisse können 1 bis 6 Minuten dauern. Sie äußern sich durch starke Dopplerverschiebungen und/oder starke Verbreiterungen von Emissionslinien im Temperaturbereich von $2 \cdot 10^{4} \mathrm{~K}$ bis $5 \cdot 10^{5} \mathrm{~K}$. Beobachtungen von Innes et al. 1997 zeigen deutlich, daß Netzwerkgebiete bei weitem mehr Energie in der Chromosphäre, Übergangsregion und Korona emittieren als Bereiche des Zellinneren. Innerhalb der Netzwerkregionen weisen hellere Bereiche mehr und stärkere Aktivität auf als dunklere, was aber nicht heißt, daß Aufhellungen stets mit Dopplerverschiebungen verbunden sind. Die beobachteten Rot- und Blauverschiebungen entsprechen Materiegeschwindigkeiten von $\approx 180 \mathrm{~km} / \mathrm{s}$. Weiterhin werden etwa zweimal mehr Blau- als Rotverschiebungen gefunden. Dies wäre verständlich, wenn es sich bei explosiven Ereignissen um Plasmaströmungen mit zwei Richtungen (engl. bi-directional plasma jets) handelte (Innes et al. 1997). Wenn sich die Quelle der Plasmaströmung in der Sonnenscheibenmitte in ca. $1000 \mathrm{~km}$ Höhe über der Photosphäre befindet (Dere 1994), so wird jede auf die Sonne hin gerichtete Strömung von der hohen Dichte des chromosphärischen und photosphärischen Materials schnell abgebremst. Der nach auswärts 
strömende Teil des Jets hingegen bewegt sich in das immer dünner werdende koronale Gas hinein. Daher ist die Blauverschiebung stärker und häufiger. Es gibt verschiedene Vermutungen über die Ursachen der explosiven Ereignisse.

Erstens könnten sie Folge eines magnetischen Feldumstrukturierungsprozesses (engl. magnetic reconnection) zwischen dem magnetischen Feld des Netzwerkes und neu auftauchenden magnetischen Schleifen sein (Dere et al. 1991). In diesem Fall müßte die gemessene Dopplergeschwindigkeit der Alfvén-Geschwindigkeit in der Chromosphäre entsprechen.

Zweitens könnte es sich um ein Phänomen von Auslöschung handeln, das durch koronale Micro-Flares hervorgerufen wird (Krucker et al. 1997).

Drittens könnte man das beobachtete Muster der explosiven Ereignisse auch mit wirbelnden Gastrichtern ähnlich irdischen Tornados erklären (Pike und Mason 1998).

Obgleich kontinuierlich bis zu 30000 explosive Ereignisse gleichzeitig stattfinden (Dere 1994), haben Abschätzungen der freigesetzten Energien ergeben, daß diese Art der Aktivität nicht die Hauptquelle für den Masse- und Energiebedarf der Chromosphäre oder der Korona sein kann (Dere et al. 1989, Dere 1994). Ihre Bedeutung liegt vielmehr in der Vermutung, daß es sich bei den beobachteten Ereignissen nur um das Hochenergie-Ende eines ganzen Spektrums von Netzwerk-Ereignissen handelt, die mit heutigen Beobachtungstechniken nicht zu erfassen sind, die aber in der Summe eben jene gesuchte Quelle darstellen könnten.

Hassler et al. 1999 beobachteten mit SUMER in der chromospärischen Si II (153,3 nm), der aus der Übergangsregion stammenden C IV (154,8 nm) und der aus der unteren Korona emittierten Ne VIII (77,0 nm) Emissionslinie Bereiche sogenannter koronaler Löcher. Es wurde schon vor ca. 30 Jahren anhand von Beobachtungen von Krieger et al. 1973 und Zirker 1977 vermutet, daß in diesen Gebieten mit seinen offenen Magnetfeldern der Ursprung des schnellen Sonnenwindes liegt. Diese Vermutung konnte mit zweidimensionalen Intensitätsbildern der oben genannten Linien und Ne VIII-Dopplergrammen bestätigt werden. Weiterhin kann man den Daten entnehmen, daß die beobachteten Ausflußgeschwindigkeiten überwiegend dem chromosphärischen Netzwerk entspringen und speziell an den Knotenpunkten der supergranularen Zellränder am stärksten sind. Das Innere der Supergranulationszellen weist hingegen entweder gar keine oder abwärtsgerichtete Strömungsgeschwindigkeiten auf.

All diese Beobachtungen unterstreichen die Bedeutung kleinskaliger Magnetfelder der ruhigen Sonne für die Struktur und Dynamik der solaren Atmosphäre bis hin zum Sonnenwind. 


\section{Theoretische Grundlagen}

\subsection{Stokes-Vektor}

Als Maxwell (1831 - 1879) die Ergebnisse von Untersuchungen über die Zusammenhänge von Magnetismus und Elektrizität von Forschern wie Oersted (1777 - 1851), Ampère (1775 - 1836) und Faraday (1791 - 1867) zusammenfaßte und mathematisch formulierte, wurde ersichtlich, daß man die entstandenen Formeln in Form einer transversalen Wellengleichung darstellen kann. Als sich dann noch herausstellte, daß sich als Geschwindigkeit für diese Welle die Lichtgeschwindigkeit aus bekannten magnetischen und elektrischen Konstanten ergab, erkannte man, daß sich Licht als elektromagnetische Welle auffassen läßt.

Die eine elektromagnetische Welle erzeugenden schwingenden Felder stehen senkrecht auf der Ausbreitungsrichtung. D.h. die die Felder beschreibenden Vektoren $\vec{E}$ und $\vec{B}$ liegen mit zunächst beliebiger Orientierung auf den zur Ausbreitungsrichtung senkrechten Wellenflächen. Wenn, wie beim natürlichen Licht (thermische Strahlung), die Richtungen der Feldvektoren auf den Wellenflächen nach dem Gesetz des Zufalls verteilt sind, spricht man von unpolarisierter Strahlung. Liegen hingegen die Feldvektoren immer in einer Richtung, so nennt man die Strahlung linear polarisiert. Elliptisch polarisiertes Licht entsteht durch Überlagerung von Licht mit verschiedenen linearen Polarisationsrichtungen. Ist ein Lichtstrahl ganz oder teilweise polarisiert, muß man im Sinne einer vollständigen Beschreibung zusätzlich zur Intensität I noch drei weitere Größen einführen. Zwei, um die zeitabhängige Orientierung des elektrischen Feldvektors $\vec{E}$ in der Wellenfläche zu beschreiben und einen weiteren, der über den Polarisationsgrad Auskunft gibt. Die Beschreibung des Vektors $\vec{E}$ reicht aus, da der magnetische Vektor $\vec{B}$ mit $|\vec{B}|=|\vec{E}|$ und $\vec{B} \perp \vec{E}$ automatisch folgt, woraus man auch gleich sieht, daß die Wahl von $\vec{E}$ willkürlich war. Obgleich die vier den Strahl beschreibenden Informationen nocheinmal anders auf die von Stokes 1852 eingeführten Parameter verteilt werden müssen, ist die Verwendung letzterer in der Praxis sinnvoller, da sie auf direkt beobachtbare Größen führen.

Betrachtet man zunächst einen einzelnen, also monochromatischen Wellenzug elektromagnetischer Strahlung, der sich in $z$-Richtung ausbreitet, so lassen sich die Komponenten des elektrischen Feldvektors in der $x-y$-Ebene darstellen wie folgt:

$$
E_{x}=A_{x} \cos \left(\omega t-\phi_{x}\right)
$$




$$
E_{y}=A_{y} \cos \left(\omega t-\phi_{y}\right) .
$$

Die $A_{x}, A_{y}$ stellen hier die Amplituden, die $\phi_{x}, \phi_{y}$ die zugehörigen Phasen und $\omega=2 \pi \nu$ die Kreisfrequenz dar. Im Falle vollständiger Polarisation - ein einzelner Wellenzug ist immer vollständig polarisiert - sind die Stokes-Parameter wie folgt definiert:

$$
\begin{gathered}
I=A_{x}{ }^{2}+A_{y}{ }^{2} \\
Q=A_{x}{ }^{2}-A_{y}{ }^{2} \\
U=2 A_{x} A_{y} \cos \left(\phi_{x}-\phi_{y}\right) \\
V=2 A_{x} A_{y} \sin \left(\phi_{x}-\phi_{y}\right)
\end{gathered}
$$

mit

$$
I^{2}=Q^{2}+U^{2}+V^{2}
$$

Der Polarisationsgrad ist nun gegeben durch:

$$
P=\sqrt{\frac{Q^{2}+U^{2}+V^{2}}{I^{2}}} .
$$

Da in der Beobachtungsrealität immer eine Überlagerung von mehreren Wellenzügen mit unterschiedlichen Amplituden und Phasen vorkommt und darüber hinaus auch ein unpolarisierter Anteil berücksichtigt werden muß, geht man wie folgt zu Mittelwerten über:

$$
\begin{gathered}
I=I^{\mathrm{unpol}}+\left\langle A_{x}{ }^{2}+A_{y}{ }^{2}\right\rangle \\
Q=\left\langle A_{x}{ }^{2}-A_{y}{ }^{2}\right\rangle \\
U=\left\langle 2 A_{x} A_{y} \cos \left(\phi_{x}-\phi_{y}\right)\right\rangle \\
V=\left\langle 2 A_{x} A_{y} \sin \left(\phi_{x}-\phi_{y}\right)\right\rangle .
\end{gathered}
$$

In diesem Fall ist $P$ nicht mehr 1 und es gilt:

$$
I \geq \sqrt{Q^{2}+U^{2}+V^{2}}
$$


Gemäß der Stokesschen Definition stellt $I$ die Gesamtintensität, $Q$ und $U$ die Intensität des linear und $V$ des zirkular polarisierten Anteils in folgenden gut beobachtbaren Termen dar:

$$
\begin{gathered}
I=\text { Gesamtintensität } \\
Q=I_{0}^{\text {linear }}-I_{90}^{\text {linear }} \\
U=I_{+45}^{\text {linear }}-I_{-45}^{\text {linear }} \\
V=I_{\text {rechts }}^{\text {zirkular }}-I_{\text {links }}^{\text {zirkular }} .
\end{gathered}
$$

Diese vier Parameter werden oft zum Stokes-Vektor $\vec{S}$ zusammengefaßt, um MatrixTransformationen mit sogenannten Mueller-Matrizen durchzuführen. Diese Matrizen beschreiben alle quantitativen Effekte von optischen Bauteilen auf den Polarisationszustand eines Lichtstrahls.

$$
\vec{S}=\left(\begin{array}{c}
I \\
Q \\
U \\
V
\end{array}\right)
$$

\subsection{Nachweis von Magnetfeldern}

\section{Sonnenspektrum}

Nachdem Planck für den Fall der Hohlraumstrahlung erstmals die Quantelung der Strahlung postulierte (1900) und Einstein 1905 den Photoeffekt deutete, indem er annahm, Licht werde in Energiepaketen, "Quanten", mit jeweils dem Energiebetrag $E=h \cdot \nu$ transportiert, war der Weg zum Verständnis des Zustandekommens von Emissions- und Absorptionslinien in Spektren geebnet. Zwanglos ließen sich jetzt die beobachteten Frequenzen der Emissions- und Absorptionslinien mit Übergängen von Elektronen zwischen festen Energiestufen $E_{n}$ des Atoms erklären, d.h. für die Spektralübergänge gilt $h\left(\nu_{2}-\nu_{1}\right)=E_{2}-E_{1}$. Die Konstante $h=6,626 \ldots \cdot 10^{-34}$ Js heißt Plancksches Wirkungsquantum und $\nu$ stellt die Frequenz dar. Diese Einsichten führten zur Aufstellung eines Termschemas für jedes Atom, bei dem jeder Term einer Energiestufe entspricht und sich die Spektrallinien durch Termdifferenzen ergeben. Jeder Linie läßt sich also ein Übergang von einem bestimmten Anregungszustand in einen anderen eines Atoms eines bestimmten Elements zuordnen. Dank Kirchhoffs und Bunsens Vermessung von Emissionslinien vieler Elemente im Labor und deren Vergleich mit den im Sonnenspektrum vorkommenden Fraunhofer-Linien war es allerdings schon Mitte des 19. Jahrhunderts möglich, Rückschlüsse über Elemente, die auf der Sonne vorkommen, zu ziehen. 


\section{Zeeman-Effekt}

Das Phänomen der Aufspaltung von Spektrallinien in zwei oder mehrere verschobene Linien, das bei der Emission von Licht aus magnetfelddurchsetzter Materie auftritt, wurde nach theoretischer Vorhersage durch Lorentz (1895) von Zeeman ein Jahr später nachgewiesen und wird deshalb Zeeman-Effekt genannt. Es werden grundsätzlich zwei Arten des Zeeman-Effekts unterschieden. Zunächst der von Lorentz mit Hilfe der klassischen Atomphysik beschriebene sogenannte normale Zeeman-Effekt, eine Triplett-Aufspaltung, die auch Lorentz-Triplett genannt wird. Diese Aufspaltung tritt in dem sehr seltenen Fall auf, wenn beide am Übergang beteiligten Zustände einen Gesamtspin von Null haben. Weit häufiger liegt jedoch der sogenannte anomale Zeeman-Effekt vor, bei dem der Gesamtspin der Zustände ungleich Null ist. In diesem Fall kann je nach betrachteter Spektrallinie eine komplizierte Multiplett-Struktur erscheinen, die mathematisch mit Hilfe der Quantenmechnik beschrieben werden kann.

Linienaufspaltungen, die auf den Zeeman-Effekt zurückgeführt werden sollen, dürfen nur durch Magnetfelder hervorgerufen werden, die so schwach sind, daß sie die $L-S$ - bzw. Russell-Saunders-Kopplung nicht aufheben, da andernfalls, je nach Kernladungszahl, der Zeeman-Effekt in den Paschen-Back-Effekt übergeht. Die in dieser Arbeit betrachteten solaren Magnetfelder sind in diesem Sinne schwach. Hier reichen die vier Quantenzahlen $L, S, J$ und $M_{J}$ zur Beschreibung der atomaren Anregungszustände aus. Die Quantenzahl $L$ steht dabei für den Gesamtbahndrehimpuls der betrachteten (äußeren) Elektronen, $S$ für den Gesamtspin, $J$ für den Gesamtdrehimpuls der Elektronen und $M_{J}$ für eine aus $J$ resultierende Komponente des magnetischen Momentes. $M_{J}$ kann die $2 J+1$ Werte von $-J,-J+1, \ldots J$ annehmen, während $J$ selbst die Werte von $|L-S|,|L-S|+1, \ldots L+S$ haben kann. Ohne äußeres Magnetfeld $B$ haben die $2 J+1$ Zustände die gleiche Energie. Sie emittieren bzw. absorbieren also Licht derselben Wellenlänge. Diese sogenannte Entartung wird durch die Anwesenheit eines äußeren Magnetfeldes aufgehoben. Für die dann entstehenden energetischen Differenzen zum Spektralterm der Grundenergie erhält man:

$$
\Delta E=g \mu_{B} M_{J} B
$$

mit dem Bohrschen Magneton:

$$
\mu_{B}=\frac{e \hbar}{2 m_{e}}
$$

und dem Landéschen g-Faktor:

$$
g=1+\frac{J(J+1)+S(S+1)-L(L+1)}{2 J(J+1)} .
$$

Die Zeeman-Aufspaltung ist dann (in SI-Einheiten):

$$
\Delta \lambda=\frac{e g^{*}}{4 \pi m_{e} c} \lambda_{0}^{2} B
$$


Weiterhin ist mit $c$ die Vakuumlichtgeschwindigkeit, mit $e$ die Elektronenladung, mit $m_{e}$ die Elektronenmasse und $\lambda_{0}$ die Wellenlänge der unverschobenen Linie gemeint. Die Größe $g^{*}$ ist gegeben durch:

$$
g^{*}=g M-g^{\prime} M^{\prime}
$$

wobei mit $g, g^{\prime}$ und $M, M^{\prime}$ die beiden Landé-Faktoren bzw. Magnetquantenzahlen der Energieniveaus des betrachteten Übergangs bezeichnet sind. Eine durch ein schwaches Magnetfeld verursachte Multiplett-Aufspaltung einer häufig nicht aufösbaren, stark druck- und Dopplerverbreiterten solaren Spektrallinie kann man behandeln als wäre sie ein Triplett. Dieser Vereinfachung trägt man Rechnung, indem man statt des $g^{*}$-Faktors einen, mit den Übergangswahrscheinlichkeiten der beteiligten Komponenten gewichteten, sogenannten effektiven Landé-Faktor benutzt.

\section{Polarisation der Zeeman-Komponenten}

Das Aufspaltungsbild einer Spektrallinie hängt auch von der Richtung des äußeren Magnetfeldes ab. Verlaufen im Falle einer vom normalen Zeeman-Effekt aufgespaltenen Absorptionslinie die Feldlinien parallel zum Sehstrahl (longitudinaler Fall), so fehlt die mittlere Komponente, und die Strahlung der beiden äußeren Komponenten ist partiell entgegengesetzt (links- bzw. rechtsdrehend) zirkular polarisiert ( $\sigma$-Komponenten). Liegen dagegen die Feldlinien senkrecht zum Sehstrahl (transversaler Fall), dann treten alle drei Komponenten auf. Die unverschobene Zentralkomponente ist in diesem Fall parallel zum Feld partiell linear polarisiert ( $\pi$-Komponente), und die beiden äußeren Komponenten sind senkrecht zur Feldrichtung partiell linear polarisiert ( $\sigma$-Komponenten). Da bei Absorptionslinien nur die verbleibende Restintensität gemessen wird, verändern sich im Falle von Emission die Polarisationseigenschaften wie folgt: Beim longitudinalen Effekt vertauschen sich rechte und linke Rotationsrichtung, beim transversalen Effekt drehen sich die linearen Polarisationsrichtungen um $90^{\circ}$.

Die Polarisationseigenschaften der Zeeman-Aufspaltung einer magnetisch empfindlichen Spektrallinie, d.h. mit möglichst großem g-Faktor, werden zu Nachweis und Messung von solaren Magnetfeldern benutzt.

\subsection{Modellrechnungen im Rahmen der Potentialfeldnäherung}

Glaubwürdige und detaillierte Informationen über das solare Magnetfeld erhält man im allgemeinen nur über die Beobachtung des Zeeman-Effekts in magnetisch sensitiven Fraunhofer-Linien. Aus diesen Messungen geht, wie im vorliegenden Fall, zumeist einzig die Sichtlinienkomponente des magnetischen Feldes auf photosphärischem Niveau hervor. Um Informationen über den Feldvektor in höheren Schichten zu bekommen, muß die vorhandene Magnetfeldkomponente geeignet extrapoliert werden. Der üblichste Weg, 
dies zu tun, ist anzunehmen, daß keine elektrischen Ströme in der Atmosphäre fließen, es sich also um ein Potentialfeld handelt. Allgemeiner ausgedrückt nimmt man an, daß das Feld oberhalb der Photosphäre kraftfrei ist. Diese Annahme wird gerechtfertigt durch die Dominanz und Stabilität des Magnetfeldes in der Chromosphäre und unteren Korona.

Im Rahmen der magnetohydrodynamischen Näherung der Plasmaphysik geht man von folgendem System linearer, partieller Differentialgleichungen aus (Kippenhahn/Möllenhoff 1973).

\section{In cgs-Einheiten:}

$$
\begin{gathered}
\nabla \times \vec{B}=\frac{4 \pi}{c} \vec{j} \\
\nabla \times \vec{E}=-\frac{1}{c} \frac{\partial \vec{B}}{\partial t} \\
\nabla \cdot \vec{B}=0 \\
\vec{j}=\lambda\left(\vec{E}+\frac{1}{c} \vec{v} \times \vec{B}\right) \\
\rho \frac{\partial \vec{v}}{\partial t}+\rho(\vec{v} \cdot \nabla) \vec{v}=-\nabla P+\frac{1}{c} \vec{j} \times \vec{B}+\rho \vec{g}+\rho \nu \Delta \vec{v} \\
\frac{\partial \rho}{\partial t}=-\nabla \cdot(\rho \vec{v}) \\
P=P(\rho, T)
\end{gathered}
$$

Die ersten drei Gleichungen sind die ersten drei Maxwell-Gleichungen, wobei bei der ersten der Verschiebungsstrom $\left(\frac{1}{c} \frac{\partial \vec{E}}{\partial t}\right)$ vernachlässigt wurde. Die Maxwell-Gleichung $\nabla \cdot \vec{E}=$ $4 \pi \sigma$ mit der Raumladung $\sigma$ wird weggelassen, da $\sigma$ in den anderen Gleichungen nicht auftritt. Die vierte Gleichung (3.27) ist das Ohmsche Gesetz unter Berücksichtigung der Plasmabewegung. $\lambda\left[\frac{1}{\mathrm{~s}}\right]$ ist die Leitfähigkeit des Materials. Die nächste Gleichung (3.28) ist die aus der Hydrodynamik übernommene Bewegungsgleichung der Materie. $\rho$ ist die Dichte, $\vec{v}$ die Materiegeschwindigkeit, $P$ der Druck, $\frac{1}{c} \vec{j} \times \vec{B}=\vec{k}$ die Kraftdichte auf die Materie (wobei der Term $\sigma \vec{E}$ vernachlässigt wurde), $\rho \vec{g}$ die Dichte der Schwerkraft mit der Schwerebeschleunigung $\vec{g}$ und $\rho \nu \Delta \vec{v}$ die Kraftdichte der Reibung mit der kinematischen Viskosität $\nu$ in $\left[\frac{\mathrm{cm}^{2}}{s}\right]$. Die sechste Gleichung (3.29) ist die Kontinuitätsgleichung der Materie. Die letzte Gleichung (3.30) stellt die Zustandsgleichung in allgemeiner Form dar.

Vereinfacht man diese Gleichungen durch die Annahme, die Größen seien alle zeitlich konstant und die Materiegeschwindigkeit Null (Hydrostatik), so folgt:

$$
\nabla \times \vec{B}=\frac{4 \pi}{c} \vec{j}
$$




$$
\begin{gathered}
\nabla \times \vec{E}=0 \\
\nabla \cdot \vec{B}=0 \\
\vec{j}=\lambda \vec{E} \\
\nabla P=\frac{1}{c} \vec{j} \times \vec{B}+\rho \vec{g}=\frac{1}{4 \pi}(\nabla \times \vec{B}) \times \vec{B}+\rho \vec{g} \\
P=P(\rho, T)
\end{gathered}
$$

Von diesem System braucht man nur die drei Gleichgungen

$$
\begin{gathered}
\nabla \cdot \vec{B}=0 \\
\nabla P=\frac{1}{4 \pi}(\nabla \times \vec{B}) \times \vec{B}+\rho \vec{g} \\
P=P(\rho, T)
\end{gathered}
$$

zu lösen, da die Größen $\vec{j}$ und $\vec{E}$ aus $\rho$ und $\vec{B}$ bestimmt werden können.

Befindet sich ein Plasma im stationären Gleichgewicht, so wird die elektromagnetische Kraft $\frac{1}{4 \pi}(\nabla \times \vec{B}) \times \vec{B}$ in der hydrostatischen Gleichung durch Druckkraft und durch äußere Kräfte kompensiert. Bei magnetohydrostatischem Gleichgewicht in einem Schwerefeld hat man

$$
\nabla P+\rho \nabla \psi_{S}=\frac{1}{4 \pi}(\nabla \times \vec{B}) \times \vec{B}
$$

mit dem Gravitationspotential $\psi_{S}\left(\vec{g}=-\nabla \psi_{S}\right)$. Unter der Vorausetzung, daß ein relativ starkes Magnetfeld in einem stark verdünnten Plasma wirkt, folgt, daß der Druck, der Druckgradient und das Gewicht eines $\mathrm{cm}^{3}$ Plasmamaterie klein sind. Das Magnetfeld muß also angenähert der Gleichung

$$
(\nabla \times \vec{B}) \times \vec{B}=0
$$

genügen. Da ein solches Feld keine Kraft auf die Materie ausübt, nennt man es kraftfrei. Die Gleichung 3.41 ist der Bedingung

$$
\nabla \times \vec{B}=\alpha \vec{B}
$$

äquivalent. $\alpha$ ist dabei eine beliebige, skalare Funktion der Ortes (und der Zeit). 
Im Rahmen der vorliegenden Arbeit wurde ein in FORTRAN77 umgeschriebenes Extrapolationsprogramm des Autors Seehafer (Seehafer 1975, Seehafer 1978, Seehafer et al. 1979) zur numerischen Berechnung der Feldlinien benutzt. Die Funktion $\alpha$, die in praktischen Anwendungen zumeist auf eine Konstante beschränkt wird, wurde hier gleich Null gesetzt. Das bedeutet

$$
\nabla \times \vec{B}=0,
$$

d.h. es fließen keine Ströme. Das Magnetfeld ist also ein Potentialfeld.

Das Extrapolationsprogramm berechnet die drei Komponenten des Magnetfelds $\left(B_{x}\right.$, $\left.B_{y}, B_{z}\right)$ an jedem Ort eines Volumes über einem gegebenen Magnetogramm nach der Methode von Seehafer wie folgt:

In einem kartesischen Koordinatensystem (x,y,z) werden die Dimensionen des zu analysierenden Bereichs mit

$$
0 \leqq x \leqq L_{x}, \quad 0 \leqq y \leqq L_{y}, 0 \leqq z<\infty
$$

bezeichnet. Die Ebene bei $z=0$ ist als Ort des aus Beobachtungen abgeleiteten Magnetogramms definiert. Dieses Magnetogramm beinhaltet schon eine Randbedingung in Form der vertikalen magnetischen Feldkomponente, $B_{z}$, bei $z=0$. Im vorliegenden Fall ist die Sichtlinienkomponente des Magnetfelds identisch mit der Normalkomponente, da in Sonnenscheibenmitte beobachtet wurde. Um eine Lösung für die Gleichung (3.42) zu finden, hat Seehafer zusätzliche Randbedingungen an den vertikalen Flächen des betrachteten Volumens eingeführt. So soll $B_{z}$ an den vertikalen Flächen $x=0, x=L_{x}$, $y=0, y=L_{y}$ verschwinden. Die Magnetfeldkomponenten lassen sich dann nach Fourier entwickeln (Seehafer 1978):

$$
\begin{gathered}
B_{x}=\sum_{m, n=1}^{\infty} \frac{C_{m n}}{\lambda_{m n}} e^{-r_{m n} z}\left\{\alpha \frac{\pi n}{L_{y}} \sin \left(\frac{\pi m x}{L_{x}}\right) \cos \left(\frac{\pi n y}{L_{y}}\right)-r_{m n} \frac{\pi m}{L_{x}} \cos \left(\frac{\pi m x}{L_{x}}\right) \sin \left(\frac{\pi n y}{L_{y}}\right)\right\} \\
B_{y}=-\sum_{m, n=1}^{\infty} \frac{C_{m n}}{\lambda_{m n}} e^{-r_{m n} z}\left\{\alpha \frac{\pi m}{L_{x}} \cos \left(\frac{\pi m x}{L_{x}}\right) \sin \left(\frac{\pi n y}{L_{y}}\right)+r_{m n} \frac{\pi n}{L_{y}} \sin \left(\frac{\pi m x}{L_{x}}\right) \cos \left(\frac{\pi n y}{L_{y}}\right)\right\} \\
B_{z}=\sum_{m, n=1}^{\infty} C_{m n} e^{-r_{m n} z} \sin \left(\frac{\pi m x}{L_{x}}\right) \sin \left(\frac{\pi n y}{L_{y}}\right)
\end{gathered}
$$

Die Fourier-Koeffizienten $C_{m n}(\mathrm{~m}, \mathrm{n} \geqq 1)$ werden durch die Gleichung (3.47) bei $z=0$ definiert. Weiterhin ist

$$
\lambda_{m n}=\pi^{2}\left(\frac{m^{2}}{L_{x}^{2}}+\frac{n^{2}}{L_{y}^{2}}\right)
$$


und

$$
r_{m n}=\sqrt{\lambda_{m n}-\alpha^{2}} .
$$

In der Arbeit von Seehafer (1978), auf die hier für weitere Details verwiesen sei, wird hervorgehoben, daß die oben beschriebene Methode es ermöglicht, das kraftfreie Magnetfeld über einer begrenzten photosphärischen Region mittels Randbedingungen an der Oberfläche des betrachteten Volumens für konstantes $\alpha$ (einschließlich des Falles $\alpha=0)$ zu berechnen. 


\section{Instrumentarium}

\subsection{Gregory-Coudé-Teleskop}

Das Gregory-Coudé-Teleskop (GCT) ist eines der deutschen Sonnenteleskope am Observatorio del Teide in Izaña auf Teneriffa. Dieses Areal, auf dem auch noch eine ganze Reihe anderer Sonnen- wie Nachtteleskope stehen, befindet sich auf ca. $2400 \mathrm{~m}$ Höhe. Dieser Standort oberhalb der Inversionsschicht hat den Vorteil, daß Beobachtungen häufig unbeeinträchtigt von den Turbulenzen und der Wolkenbildung der unteren Troposphäre bleiben. Das optische System des Teleskops befindet sich, um möglichst weit von den sich durch Bodenerwärmung bildenden Szintillationen der Luft entfernt zu sein, auf einem ca. $20 \mathrm{~m}$ hohen Turm. Wie der Name schon sagt, handelt es sich um ein Spiegelteleskop in der Gregory-Coudé-Bauweise.

Durch ein Eintrittsfenster von knapp einem halben Meter Durchmesser dringt das von der Sonne kommende Licht in einen ca. $6 \mathrm{~m}$ langen, evakuierten Aluminiumtubus ein. Dieser Tubus ist auf einer Säule asymmetrisch, parallaktisch in sogenannter deutscher Montierung gelagert. Das Licht trifft nun auf den dem Eintrittsfenster gegenüber liegenden parabolischen Hauptspiegel von $45 \mathrm{~cm}$ Durchmesser und 2,40 m Brennweite. Im Primärfokus (F1) befindet sich eine Blende, die nur den kleinen interessierenden Teil des Sonnenbildes durchläßt und den Rest, um Streulicht und Erwärmung zu vermindern, durch ein sogenanntes "Hitzefenster" aus dem Tubus herausspiegelt. Das verbleibende Licht fällt nun auf den, zum Fokussieren verschiebbaren, elliptischen Sekundärspiegel, der ein Brennweitenverhältnis von 1 zu 10 hat. Die effektive Brennweite der Primär- und Sekundäroptik beträgt $25 \mathrm{~m}$. Das Licht wird dann zunächst mit Hilfe der verspiegelten Rückseite der schon erwähnten Blende (Umlenkspiegel Nr.1) aus dem Teleskoptubus heraus, durch eine $\lambda / 2$-Phasenverzögerungsplatte hindurch auf den das Licht in die Stundenachse lenkenden Umlenkspiegel (Nr.2), den sogenannten Coudé-Spiegel, geworfen. Durch das Einfügen der $\lambda / 2$-Phasenverzögerungsplatte zwischen die beiden Umlenkspiegel werden die beiden Polarisationsebenen in die Richtung wie zur Zeit der Äquinoktien gedreht und der Zustand minimaler instrumenteller Polarisation erreicht. Während der Äquinoktien ist das Instrument intrinsisch polarisartionsfrei, da die Umlenkspiegel (Nr.1 und Nr.2) in Deklination und Rektaszension jeweils um $90^{\circ}$ reflektieren und die von ihnen eingeführten linearen Polarisationen senkrecht aufeinander stehen. Das Licht in die Stundenachse des Teleskops zu leiten und damit einen raumfesten Fokus zu erhalten, 
hat den Vorteil, daß man große raumfeste Spektrographen verwenden kann, jedoch auch den Nachteil eines um $15^{\circ}$ pro Stunde rotierenden Sonnenbildes. Durch ein kleines Austrittsfenster von ca. $10 \mathrm{~cm}$ Durchmesser verläßt das Licht den evakuierten Bereich.

\subsubsection{Optischer Aufbau}

\section{Präfokusinstumente}

Das vom Austrittsfenster kommende Licht passiert den Bowen-Kompensator des Bildrotators und dessen drei Spiegel. Der Bowen-Kompensator, der die, durch die Reflexionen an den Spiegeln entstehende, zusätzliche Polarisation soweit wie möglich kompensiert, besteht aus zwei stufenlos gegeneinander verdrehbaren $\lambda / 8$-Platten. Die schon erwähnten drei Spiegel des Bildrotators oder besser Bild-Derotators können durch gemeinsame Rotation um die optische Achse das Sonnenbild drehen. Diese Eigenschaft findet Verwendung, wenn, wie im vorliegenden Fall, eine vorgegebene Orientierung des Sonnenbildes bezüglich des Spektrographeneintrittsspaltes gegen die dem Gregory-Coudé-System intrinsische Bilddrehung gehalten werden soll. Als nächstes durchstrahlt das Licht den Bowen-Kompensator des Bildfeldscanners und den Scanner selbst, der wie der Bildrotator aus drei Spiegeln besteht, nur, daß statt einer gemeinsamen Drehung eine gemeinsame lineare Verschiebung der Spiegel hier zu einem Bildversatz führt. Es ist also mit dem Scanner möglich, das Sonnenbild über den Eintrittsspalt des Gitterspektrographen zu schieben, ohne das sehr viel schwerere und somit trägere Teleskop zu bewegen. Der Bowen-Kompensator hat hier den gleichen Aufbau und die gleiche Aufgabe wie beim Bildrotator. Das Licht erreicht den auf den verspiegelten Spaltbacken liegenden zweiten Brennpunkt (F2) des elliptischen Sekundärspiegels. Das von den Spaltbacken reflektierte Licht versorgt über einen Umlenkspiegel die sogenannte Spaltbeobachtungsanlage. Sie stellt dem Beobachter zur besseren Orientierung ein Weißlichtbild, ein Ca II $K$ und ein $\mathrm{H} \alpha$-Filtergramm zur Verfügung.

\section{Postfokusinstumente}

Bevor das durch den Spalt gelangte Licht den Czerny-Turner-Spektrographen erreicht, muß es noch die Phasenverzögerungsplatte und die beiden doppelbrechenden Kalkspatkristalle des Polarimeters sowie den, den ausgewählten Spektralbereich eingrenzenden, Interferenzfilter passieren. Das Licht fällt also zuerst auf die dem Polarimeter vorangestellte $\lambda / 4$-Phasenverzögerungsplatte. Dadurch wird zirkular polarisiertes in linear polarisiertes Licht umgewandelt. Umgekehrt wird natürlich auch linear polarisiertes Licht in zirkular polarisiertes verwandelt, während unpolarisiertes Licht die $\lambda / 4$-Platte unverändert passiert. Danach trifft dieses Licht auf den ersten Kalkspatkristall, der für zwei aufeinander senkrecht stehende optische Ebenen unterschiedliche Brechungsindizes hat. Diese beiden Ebenen sind um $+45^{\circ}$ bzw. $-45^{\circ}$ gegen die schnelle Achse der $\lambda / 4$-Platte gedreht. Aufgrund der Doppelbrechung spaltet sich das einfallende Licht 
in seine zwei unterschiedlich linear polarisierten Anteile räumlich auf. Man nennt den einen Strahl den ordentlichen und den anderen den außerordentlichen. Die resultierenden, unterschiedlich langen, optischen Wege der Teilstrahlen werden im zweiten, dem ersten völlig identischen, jedoch um $90^{\circ}$ verdrehten, Kristall ausgeglichen. Durch diesen Aufbau gelingt es, die rechts- bzw. links zirkular polarisierten Anteile des Lichts räumlich zu trennen und so die beiden, beim longitudinalen Zeeman-Effekt entstehenden, $\sigma$-Komponenten unabhängig voneinander aufzuzeichnen (Stokes- $V$-Polarimetrie). Man erhält also bei idealem Verhalten aller Komponenten und idealer Justierung die beiden Kanäle $\frac{1}{2}(I+V)$ und $\frac{1}{2}(I-V)$, aus denen sich das Stokes- $I$-Signal durch Addition und das Stokes- $V$-Signal durch Subtraktion ergibt. Wollte man das Stokes- $Q$ bzw. Stokes$U$-Signal erhalten, so müsste eine $\lambda / 2$-Platte anstelle der $\lambda / 4$-Platte eingefügt bzw. die $\lambda / 4$-Platte entfernt werden. Nach dem schon erwähnten Interferenzfilter dringen die beiden Teilstrahlen in den Spektrographenraum ein, wo sie nach $10 \mathrm{~m}$ (Fokallänge des Czerny-Turner-Spektrographen) und zwei Umlenkspiegeln auf einen Kollimator fallen. Der Kollimator leuchtet mit dem nun parallelen Lichtbündel das $180 \mathrm{~mm} \times 360 \mathrm{~mm}$ große Reflexionsgitter vollständig aus. Das Gitter zerlegt mit einem Blaze-Winkel von $63^{\circ}, 5$ und 316 Strichen/mm das Licht spektral und reflektiert es über einen beweglichen Kameraspiegel (mit ihm kann man das Spektrum fokussieren) und einen weiteren Umlenkspiegel zum Spektrographenausgang. Dort befindet sich eine FlameStar II CCD-Kamera (Charge-Coupled-Device) der Firma LaVision mit einem Peltier-gekühlten TH7863FT CCD-Chip der Firma Thomson. Dieser Detektor besteht aus $384 \times 286$ quadratischen Pixeln von $23 \mu \mathrm{m}$ Kantenlänge.

\subsection{Vakuum-Turm-Teleskop}

Das Vakuum-Turm-Teleskop (VTT) ist ein 38 Meter hohes Sonnenteleskop, das vom Kiepenheuer-Institut in Freiburg betrieben wird. Es steht neben dem GCT am Observatorio del Teide in Izaña auf Teneriffa und profitiert somit in bezug auf die Sichtbedingungen ebenso von der Insellage und der damit verbundenen homogenen Luftschichtung des Standortes. Durch zwei computergesteuerte Coelostatenspiegel wird das Sonnenlicht senkrecht durch eine Eintrittsöffnung von $70 \mathrm{~cm}$ Durchmesser in ein Stahlrohr von 2,5 m Durchmesser gelenkt, an dessen Boden der Hauptspiegel mit einer Brennweite von $46 \mathrm{~m}$ befestigt ist. Das Stahlrohr ist zur Verringerung interner durch Erwärmung verursachter Luftströmungen und Turbulenzen evakuiert. Innerhalb dieses Stahlrohrs wird der Strahlengang zweimal gefaltet und dann durch ein Austrittsfenster geführt. Von dort kann das Licht entweder horizontal in Laboratorien oder vertikal in einen hochauflösenden Echelle-Spektrographen gelenkt werden. Das Sonnenlicht wurde im vorliegenden Fall auf einen Lyot-Filter gelenkt und anschließend mit einer 1024 Pixel mal 1024 Pixel CCD-Kamera aufgenommen. Mit dem schnellen Verschluß der Kamera können in rascher Folge kurzzeitbelichtete Filtergramme erzeugt werden. 


\subsection{Solar Ultraviolet Measurements of Emitted Radiation}

\section{Solar and Heliospheric Observatory}

Die Raumsonde SOHO (Solar and Heliospheric Observatory) mit dem UV-Teleskop SUMER (Solar Ultraviolet Measurements of Emitted Radiation) an Bord wurde am 2.12.1995 mit einer Atlas II-AS Centaur Rakete gestartet und erreichte im Februar 1996 den Lagrangepunkt L1. Der L1 ist derjenige Librationspunkt, der auf der Verbindungsgraden Erde-Sonne liegt. An diesem Punkt, 1,5 Mio. km von der Erde entfernt, sind die Gravitations- und Fliehkräfte von Erde und Sonne auf einen korotierenden Körper gerade im Gleichgewicht. Die dreiachsenstabilisierte Sonnenforschungssonde umkreist den L1 in einem Haloorbit alle 6 Monate, wobei sie stets auf die Sonne gerichtet bleibt. Dieser Haloorbit hat eine Ausdehnung von ca. $200000 \mathrm{~km}$ in der Richtung Erde-Sonne, ca. $650000 \mathrm{~km}$ in der Ekliptik und $200000 \mathrm{~km}$ senkrecht zur Ekliptik. SOHO hat eine Masse von $1861 \mathrm{~kg}$, wovon $655 \mathrm{~kg}$ auf zwölf wissenschaftliche Experimente entfallen:

SUMER (Solar Ultraviolet Measurements of Emitted Radiation).

GOLF (Global Oscillations at Low Frequencies)

VIRGO (Variability of Solar Irradiance and Gravity Oscillations)

MDI/SOI (Michelson Doppler Imager/Solar Oscillations Investigation)

CDS (Coronal Diagnostic Spectrometer)

EIT (Extreme-Ultraviolet Imaging Telescope)

UVCS (UltraViolet Coronagraph Spectrometer)

LASCO (Large Angle Spectroscopic Coronagraph)

SWAN (Solar Wind Anisotropies)

CELIAS (Charge Element and Isotope Analysis System)

COSTEP (Comprehensive Supra Thermal and Energetic Particle Analyser)

ERNE (Energetic and Relativistic Nuclei and Electron Experiment)

Die Solarzellen der Sonde stellen die elektrische Leistung von $1400 \mathrm{~W}$ zur Verfügung. Die mit den verschiedenen Experimenten gewonnenen Daten können entweder in den 2 Gbit Festspeicher oder dem 1 Gbit Datenband an Bord der Sonde zwischengelagert werden, bis sie dann mit einer Telemetrie-Rate von bis zu knapp $250 \mathrm{kbits} / \mathrm{s}$ zur Erde gefunkt werden. Mehr zu der Sonde und ihren Instrumenten findet sich in dem "Solar Physics"-Band 162 von 1995.

\section{Solar Ultraviolet Measurements of Emitted Radiation}

Das SUMER-Instrument (Wilhelm et al. 1995) ist für die Beobachtung solarer EUVEmissionslinien (Extreme Ultraviolett) gebaut worden. Diese Linien entstehen in der Chromosphäre, Übergangsregion und unteren Korona der Sonne bei Elektronentemperaturen zwischen $5 \cdot 10^{3} \mathrm{~K}$ bis zu einigen $10^{7} \mathrm{~K}$ in aktiven Gebieten. Der abbildende Spektrograph des Instruments hat ein räumliches Auflösungsvermögen von ca. $1^{\prime \prime}$ und 
ein spektrales Auflösungselement von $\approx 4,4 \mathrm{pm}$ in erster und $\approx 2,2 \mathrm{pm}$ in zweiter Ordnung. Die beiden Detektoren von SUMER werden im wesentlichen für die Beobachtung des Lichtes im Wellenlängenbereich von $46,5 \mathrm{~nm}$ bis $161,0 \mathrm{~nm}$ genutzt. Es ist hierbei anzumerken, daß nur wenige Linien unterhalb von 50,0 nm bisher mit SUMER beobachtet wurden.

\subsubsection{Optischer Aufbau}

Das von der Sonne kommende Licht fällt durch die Eintrittsöffnung des Aluminiumgehäuses von SUMER auf den um zwei Achsen schwenkbaren parabolischen Primärspiegel. Die beiden Achsen des Spiegels lassen sich um $\pm 32^{\prime}$ bewegen, so daß ein dynamisches Sichtfeld von $64^{\prime} \times 64^{\prime}$ erreicht wird. Die kleinste Schrittweite in Nord-Süd- und Ost-West-Richtung beträgt 0," 375. Das Teleskop hat eine Brennweite von 1302, $77 \mathrm{~mm}$ bei einem Öffnungsverhältnis von 10,67. Dieser Wert gilt bei $80^{\circ} \mathrm{C}$, der Betriebstemperatur des Teleskopspiegels. Auf dem Weg zum Primärspiegel, der wie alle anderen optischen Teile von SUMER aus Siliziumkarbid ( $\mathrm{SiC}$ ) besteht, passiert das Licht zwei streulichtreduzierende Blenden, zwischen denen eine elektrostatische Ablenkvorrichtung dafür sorgt, daß keine Protonen des Sonnenwindes in das Teleskop eindringen und den Primärspiegel beschädigen. Der vom Spiegel kommende Lichtstrahl trifft zunächst auf einen Vorspalt und wird dann auf der Eintrittsebene des Spektrometers fokussiert. Der Beobachter kann an dieser Stelle zwischen vier möglichen Einstellungen $\left(1^{\prime \prime} \times 300^{\prime \prime}\right.$; $\left.1^{\prime \prime} \times 120^{\prime \prime} ; 0,3^{\prime \prime} \times 120^{\prime \prime} ; 4^{\prime \prime} \times 300^{\prime \prime}\right)$ des Spaltes wählen. In der Spaltebene beträgt der Abbildungsmaßstab 6, 316 $\frac{\mu \mathrm{m}}{\prime \prime}$. Ein Teil des Lichtes wird hinter dem Eintrittsspalt auf eine Spaltkamera weitergeleitet. Sie deckt den Wellenlängenbereich von 577-652 nm ab und besitzt bei $25 \frac{\mu \mathrm{m}}{\text { Pixel }} 512$ Pixel für die Ortsrichtung. Der Hauptteil des vom jeweiligen Spalt durchgelassenen Lichtes wird von einem weiteren parabolischen Spiegel, dem Kollimator, nun als kollineares Lichtbündel durch einen Lyot-Filter geführt. Der Kollimator hat eine Brennweite von 399,60 mm. Das vom Lyot-Filter durchgelassene Licht fällt auf einen planen Kippspiegel zur Abtastung (engl. scan mirror). Dieser läßt sich, um den ganzen spektralen Bereich abdecken zu können, um 8, 4 bis $17,{ }^{\circ} 5$ bezüglich des einfallenden Lichtstrahls drehen. Der plane Scan-Spiegel reflektiert das Licht auf das ebenfalls aus Siliziumkarbid bestehende sphärisch-konkave Gitter. Dieses Gitter besitzt 3600,42 Linien pro Millimeter und die sphärisch-konkave Oberfläche hat einen Radius von 3 200,78 mm. Spektrallinien erster und zweiter Ordnung werden gleichzeitig auf einem der beiden zweidimensionalen in der Fokalebene des Gitters nebeneinander befindlichen Detektoren (A oder B) abgebildet und aufgezeichnet. Die Detektoren bestehen aus 27,0 $\times 9,5 \mathrm{~mm}^{2}$ großen Multikanalplatten, deren Signale elektronisch in 1024 spektralen $\times 360$ räumlichen Pixeln unterteilt werden. Auf diese Weise erhält man eine mittlere Pixelgröße von 26,5 × 26,5 $\mathrm{m}^{2}$. Die beiden Detektoren A und B, von denen jeweils nur einer zur Zeit genutzt werden kann, decken, obwohl von gleicher Bauart, einen leicht unterschiedlichen Wellenlängenbereich ab, da der Detektor B anders als A mit einem Abstand von $\Delta=70,4 \mathrm{~mm}$ von der Gitternormalen entfernt aufgestellt wurde. 
Die vorliegenden Beobachtungen wurden mit Detektor A angefertigt. Der empfindlichste Bereich des Detektors ist die zentrale mit Kaliumbromid (KBr) bedampfte Fläche (auf Detektor A die spektralen Pixel 280-770 und bei B die spektralen Pixel 270-758). Daran schließen sich rechts und links, in spektraler Richtung, symmetrisch zwei unbedampfte Detektorflächen (bare MCP [multichannel plates]) an. Abgeschlossen wird die Detektorfläche rechts und links mit zwei dünnen Streifen, die für die Beobachtung der starken Lyman- $\alpha$-Linie geeignet sind. 


\section{Beobachtungen und Datenreduktion}

\subsection{Beobachtungskampagne}

Die Daten, auf die in dieser Arbeit Bezug genommen wird, wurden am 10.5.1999 im Rahmen einer internationalen, koordinierten Beobachtungskampagne gewonnen. An dieser Kampagne nahmen das GCT (Gregory-Coudé-Teleskop), das VTT (Vakuum-TurmTeleskop) und SUMER (Solar Ultraviolet Measurements of Emitted Radiation) teil. Es wurde ein kleines Gebiet mit marginaler magnetischer Aktivität in der Sonnenscheibenmitte (in SOHO-Koordinaten bei $x=-20, y=-10$ ) als Ziel ausgewählt. An jedem Teleskop wurde zu Beginn der Beobachtung gleichzeitig ein großformatiges Übersichtsbild (Kontextbild) erzeugt. Mit diesen Bildern sollte die räumliche Anpassung (engl. alignment) der Bilder der verschiedenen Teleskope unterstützt werden. Anschließend verfolgten die drei Teleskope die ausgewählte Region über viereinhalb Stunden und zeichneten dessen zeitliche und räumliche Dynamik in einem gleichmäßigen Abstand auf. Die Beobachtungen sind in diversen Spektralbereichen ausgeführt worden, so daß bei Berücksichtigung der verschiedenen Entstehungshöhen der unterschiedlichen Wellenlängenintervalle ein räumliches Bild der Region entsteht.

\subsection{Daten vom Gregory-Coudé-Teleskop}

Am GCT wurde von 8:09 UT bis 12:35 UT (universal time) zweidimensionale Stokes- $V$ Polarimetrie (Stolpe et al. 1997; Stolpe 1998) in der photosphärischen Absorptionslinie des neutralen Eisens (Fe I) bei 630,25 nm durchgeführt. Diese von zwei terrestrischen $\mathrm{O}_{2^{-}}$ Linien spektral eingeschlossene Linie reagiert bei einem Landé-Faktor von $g_{\text {eff }}=2,5$ äußerst sensibel auf Magnetfelder und ist somit zu deren Nachweis sehr geeignet. Die verwendete Spaltbreite entsprach mit $120 \mu \mathrm{m}$ einer Bogensekunde, einem Winkel, der auf der Sonne $725 \mathrm{~km}$ überstreicht. Die Schrittweite von einem Spektrum zum nächsten wurde, um eine vollständige Abdeckung zu erreichen, mit $1^{\prime \prime}$ der Spaltbreite angepaßt. Um die beiden Kanäle $\frac{1}{2}(I \pm V)$ voneinander deutlich getrennt gleichzeitig auf dem Detektor-Chip darzustellen, mußten die Spaltbegrenzer so eingestellt werden, daß sich für die Spalthöhe 32" ergaben. Die ursprünglich 384 Pixel für die beiden Kanäle in Ortsrichtung wurden aus Speicherkapazitätsgründen durch Summation jeweils zweier benachbarter Pixelwerte um den Faktor 2 gestaucht. Somit entfallen also rechnerisch 96 und 
nach Abzug der Rand- und Trennpixel effektiv 84 Pixel auf ca. 32" (84 Pixel.2 (binning factor) $\cdot 23 \frac{\mu \mathrm{m}}{\text { Pixel }} \cdot \frac{1}{120} \frac{\prime \prime}{\mu \mathrm{m}} \approx 32^{\prime \prime}$ ), so daß man eine Ortsauflösung von ca. $0,38 \frac{\prime \prime}{\text { Pixel findet, was }}$ bei der benutzten Spaltbreite immer noch bei weitem ausreicht. Die 286 Pixel in spektraler Richtung führen bei $0,354 \cdot 10^{-12} \frac{\mathrm{m}}{\text { Pixel }}$ auf ein abgebildetes Intervall von ca. $0,10 \mathrm{~nm}$. Jeder Scan besteht aus 60 Spektren, wobei die Belichtungszeit pro Spektrum 0,3 s betrug. Alle 2 Minuten wurden jeweils zwei Scans, die später zu einem Bild zusammengesetzt wurden, aufgenommen. Ein solches Bild hat dann eine Fläche von $60^{\prime \prime} \times 57^{\prime \prime}$.

\subsubsection{Ausgangslage}

Als erstes wurden mit geschlossenem Spektrographenausgang zwei Darkscans, eines mit 60 und das andere mit 100 Schritten des Bildfeldcanners, aufgenommen. Für das Kontextbild wurden sechs Scans mit jeweils 100 Schritten so aufgenommen, daß sie später zu einem großen Gesamtbild von $100^{\prime \prime} \times 157^{\prime \prime}$ zusammengesetzt werden konnten. Nach jedem Schritt eines Scans wurden die beiden Kanäle $\frac{1}{2}(I \pm V)$ aufgezeichnet. Es folgte die eigentliche Beobachtungsserie, in deren Verlauf 240 Scans von jeweils 60 Schritten aufgenommen wurden. Am Ende der Kampagne wurden Dark- und Baummusterscans (jeweils zwei 60er und ein 100er) sowie 10 Flatfieldscans mit 60 und 10 mit 100 Schritten aufgezeichnet. Die gesamte erhobene Datenmenge belief sich am Ende des Tages auf knapp zwei Gigabyte verteilt auf 275 Dateien.

\subsubsection{Datenverarbeitung}

\section{Dunkelbild}

Die zur Datenaufzeichnung benutzten Bildelemente (engl. pixel) des zweidimensionalen CCD-Chips der Kamera haben ein gewisses, jeweils unterschiedliches, Hintergrundsignal. Dieses sogenannte Nullsignal ist vornehmlich auf die Erwärmung des Halbleitermaterials, bedingt durch das auf ihn fallende Sonnenlicht, die Raumtemperatur und den Betrieb d.h. Aufzeichnen und Auslesen desselben, aber auch auf die Nullpunktverschiebung (engl. offset) der nachgeschalteten Elektronik zurückzuführen. Obgleich der Chip gekühlt und somit das thermische Rauschen stark reduziert wird, verbleibt ein Rest, der im Nachhinein durch sogenannte Dunkelbilder (engl. darks) korrigiert werden muß. Diese Korrekturbilder werden erzeugt, indem man die Spektrographenöffnung, durch die das Licht der Sonne auf die Kamera fällt, verschließt und den CCD-Chip ausließt, wobei wichtig ist, daß die Belichtungszeit dieses Bildes genauso lang wie die der aufgenommenen und zu korrigierenden Spektren ist, da das Dunkelsignal von der Integrationszeit abhängt. Um der statistischen Natur des Signals gerecht zu werden, ist es sinnvoll, über eine größere Anzahl von Dunkelbildern zu mitteln. 


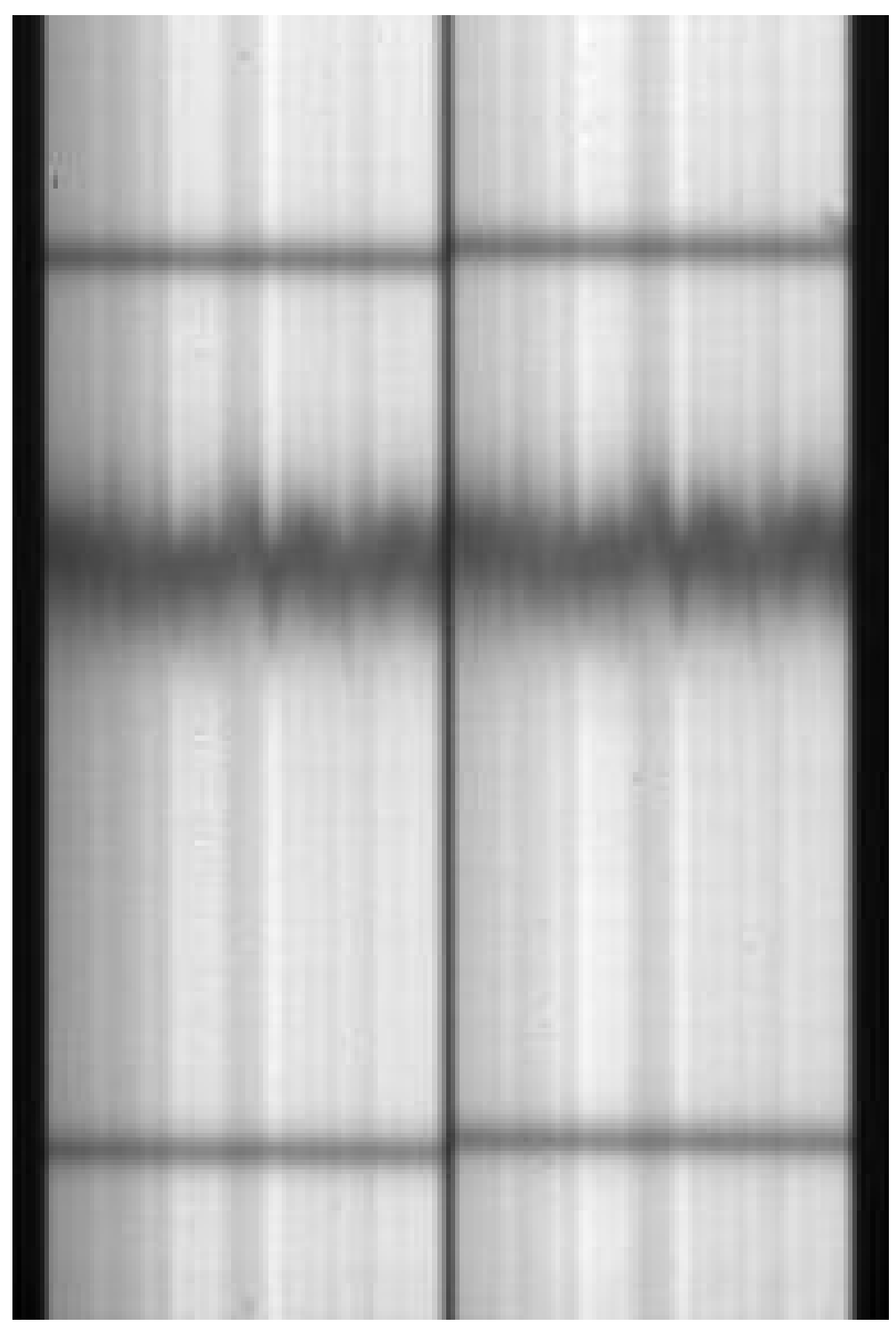

Abbildung 5.1: Typisches Rohdatenbild vom GCT. Man sieht die beiden $\frac{1}{2}(I \pm V)$ Kanäle, die durch die vertikale schwarze Linie in der Bildmitte voneinander getrennt werden. Die Fe I-Absorptionslinie bei $630,25 \mathrm{~nm}$ findet sich in beiden Kanälen als dicke schwarze unregelmäßige horizontale Linie, die auf jeder Seite durch dünne $\mathrm{O}_{2}$-Linien terrestrischen Ursprungs spektral eingerahmt wird. In Ortsrichtung (horizontal) besteht das Datenfeld aus 196 Pixeln, wobei pro Kanal effektiv 84 Pixel zur Verfügung stehen, was $32^{\prime \prime}$ entspricht. Die 286 Pixel in Dispersionsrichtung entsprechen bei $0,354 \cdot 10^{-12} \frac{\mathrm{m}}{\text { Pixel }}$ einem Intervall von ca. $0,1 \mathrm{~nm}$. Die Wellenlänge wird nach oben hin größer. 


\section{Flatfield und Verstärkungsmatrix}

Neben dem Problem des Dunkelrauschens gibt es noch weitere störende Einflüsse, wie z.B. unterschiedliche Quantenausbeute der einzelnen Pixel, Verschmutzung des CCDChips der Kamera, Staub auf den optischen Oberflächen des Strahlengangs und Ausleuchtungsunterschiede. Um diese Effekte zu korrigieren, mißt man die Pixelempfindlichkeit unter Einschluß aller Störeffekte. Theoretisch wäre dazu eine homogene Lichtquelle in Form einer Lampe ideal. In der Praxis kommt man dieser Forderung jedoch sehr nah, indem man für jede Scannerposition separat eine ganze Reihe von Bildern, entweder mit defokussiertem oder, wie im vorliegenden Fall, schnell bewegtem Teleskop erzeugt und anschließend mittelt. Die, auf diese Weise erhaltenen, strukturlosen Intensitätsbilder nennt man Flatfields. Die Methode des Defokussierens hat gegenüber der anderen den Nachteil, daß sich der Einfallswinkel der Lichtstrahlen und damit die Position der auf den Chip projizierten Staubkörner verändert, so daß nach Behandlung der Rohbilder mit den Korrekturbildern kleinere Artefakte verbleiben. Jetzt zieht man von den Flatfields noch jeweils das Dunkelbild ab und erhält für jede Scannerposition Korrekturbilder, die nur noch von ihrer spektralen Information befreit werden müssen, um ihrer anfangs beschriebenen Aufgabe gerecht zu werden. Zu diesem Zweck wird zunächst ein Polynom zweiten Grades an das Profil der Fe I-Absorptionslinie einer jeden Ortsposition (entlang des Spaltes) eines Flatfield-Spektrums angepaßt. Mit Hilfe dieser Polynome ermittelt man die relative Minimumposition (in Wellenlängenrichtung) und merkt sich diese für alle Ortspositionen in einer Liste. Nun werden die Profile gemäß der Minimum-PositionsListe in spektraler Richtung übereinander geschoben und anschließend das mittlere Profil bestimmt. Das auf diese Weise errechnete mittlere Profil wird nun wieder mit Hilfe der Minimum-Positions-Liste an den verschiedenen Ortspositionen eingesetzt, so daß man am Ende ein geglättetes, künstliches Spektrum erhält. Die Verstärkungsmatrix (engl. gain table) entsteht, indem man für jede Position des Scanners separat das um das Dunkelrauschen reduzierte Flatfield durch das jeweils zugehörige künstliche Spektrum teilt. Die fertige Verstärkungsmatrix enthält keine spektrale Information mehr, sondern nur noch, wie gefordert, alle Verunreinigungen und Störsignale.

\section{Korrelation der beiden Kanäle}

Die vom Polarimeter erzeugten beiden Kanäle $\frac{1}{2}(I \pm V)$ werden im Laufe der Auswertung addiert bzw. subtrahiert, um das Stokes- $I$ bzw. Stokes- $V$-Signal zu ermitteln. Es ist daher eminent wichtig, sicher zu stellen, daß man immer die jeweils gleichen Orte bzw. Wellenlängen miteinander in Beziehung bringt. Um die etwaigen Verschiebungen der Kanäle gegeneinander bzw. Verzerrungen innerhalb der Teilspektren zu bestimmen, wurde mit Hilfe der Kreuzkorrelation für jede Bildposition jeder Scannerposition des einen Kanals ein zweidimensionaler Verschiebungsvektor bezüglich des anderen errechnet. Für die sich aus den Verschiebungsvektoren zusammensetzende Verzeichnungskarte (engl. distortion map) wurden in Dispersionsrichtung die beiden, die Fe I-Linie einschlie- 


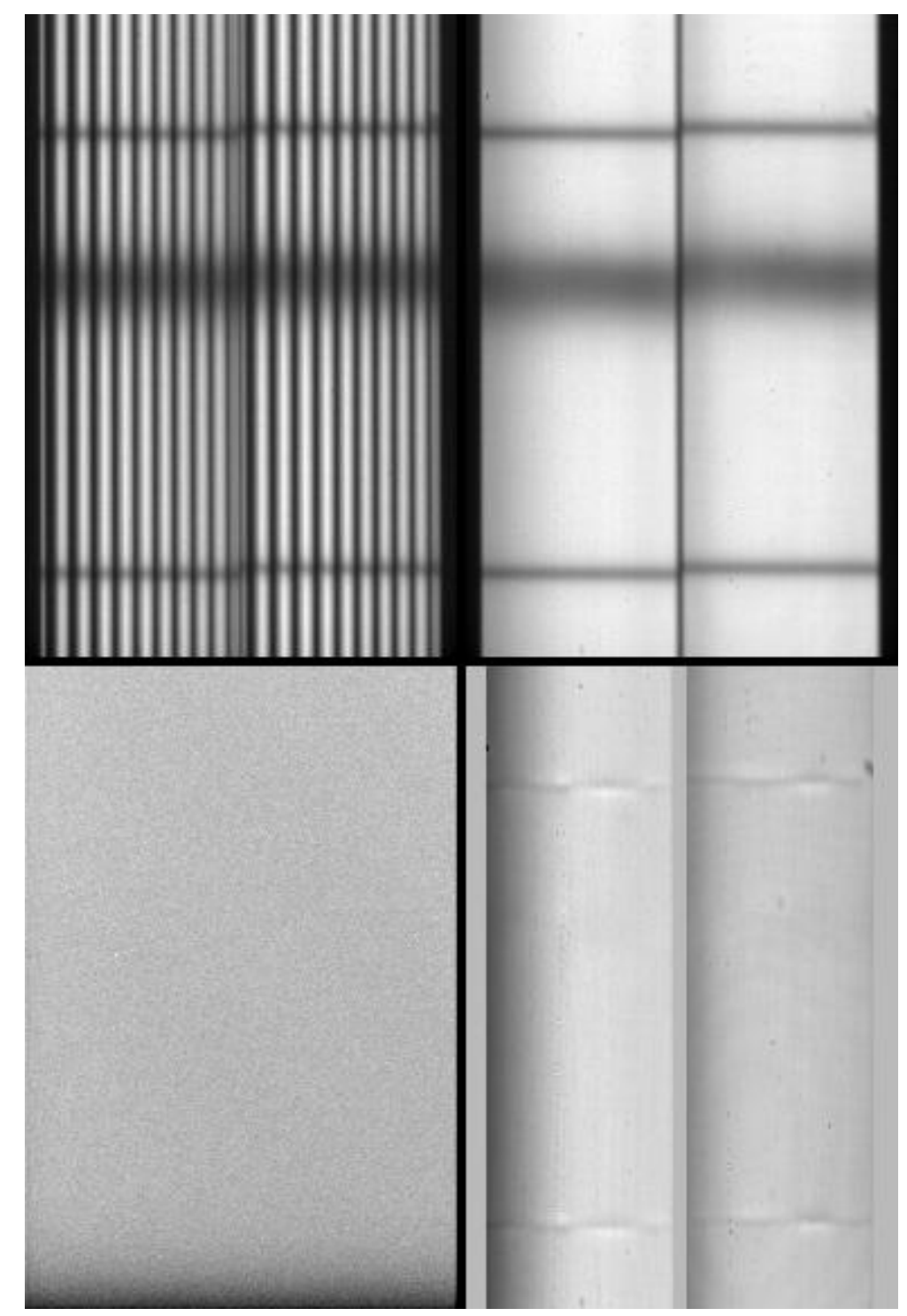

Abbildung 5.2: Oben links ist ein typisches Baummusterbild abgebildet. Daneben findet sich ein Flatfield. Unten links ist ein Dark abgebildet, daneben eine typische Gaintable. 
ßenden, terrestrischen $\mathrm{O}_{2}$-Linien zur Orientierung genutzt. Für die Ortsrichtung mußten extra sogenannte Baummusterbilder aufgenommen werden. Das sind Bilder, die man erhält, wenn man während einer Flatfield-Aufnahmeserie ein Streifenmuster quer über den Eintrittsspalt legt. Es entstehen dann Flatfields mit sehr dunklen Streifen in Ortsrichtung. Um bessere Ergebnisse bei der Korrelation zu erhalten, wurde der Kontrast dieser Bilder gemindert, indem "normale" Flatfields zum Baummusterbild hinzuaddiert und anschließend das Ganze gemittelt wurde.

\section{Rauschfilterung}

Da für die spätere Auswertung vor allem Größen, die aus spektraler Information gewonnen wurden, entscheidend sind, ist eine sorgfältige Rauschfilterung in Dispersionsrichtung, anders als in Ortsrichtung sehr wichtig. Hierzu wird das verrauschte Signal fouriertransformiert und im Fourier-Raum mit einem Tiefpaßfilter multipliziert. Das entrauschte Signal erhält man anschließend durch Rücktransformation in den Ortsraum. Die zwei für die Berechnung des Tiefpaßfilters wichtigen Parameter Filtersteilheit und Grenzfrequenz werden an exemplarischen Spektren interaktiv bestimmt und durch Vergleich von gefiltertem zu ungefiltertem Profil kontrolliert.

\section{Bearbeitung der Rohspektren}

Nachdem die Erstellung der Hilfs- bzw. Korrekturbilder (Dark, Flatfield, Gaintable und Distortion Map) dargestellt und die Rauschfilterung erklärt wurde, sollen jetzt des besseren Überblicks halber noch die wichtigsten Schritte vom Rohspektrum zum für die physikalische Auswertung fertigen Spektrum aufgezeigt werden. Die Indizes i und j beziehen sich auf die Zeilen bzw. Spalten des Detektors.

Als erstes wird vom Rohspektrum $\tilde{I}(i, j)$ das gemittelte Dunkelbild $D(i, j)$ abgezogen und das Ganze dann durch die, der jeweiligen Scannerposition entsprechende, Verstärkungsmatrix $G(i, j)$ geteilt.

$$
I(i, j)=\frac{\tilde{I}(i, j)-D(i, j)}{G(i, j)}
$$

Als nächstes wird das Spektrum $I(i, j)$ durch den Mittelwert des angrenzenden Kontinuums geteilt d.h. normiert. Danach wird das normierte Spektrum der Tiefpaßfilterung im Fourier-Raum unterworfen. Zuletzt werden die beiden Kanäle des Spektrums mit der Verzeichnungskarte aufeinander abgestimmt.

\subsubsection{Physikalische Auswertung}

Aus den beiden Kanälen $\frac{1}{2}(I \pm V)$ eines jeden Spektrums läßt sich das Stokes- $I$-Signal durch Addition und das Stokes- $V$-Signal durch Subtraktion jetzt leicht bestimmen. Je 


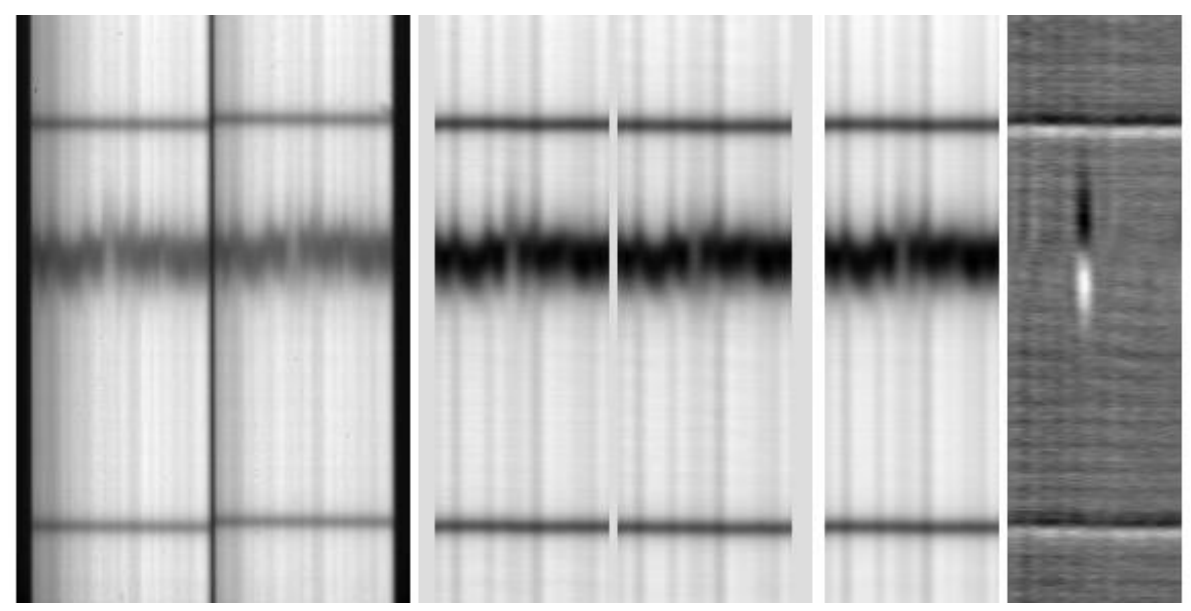

Abbildung 5.3: Ganz links erkennt man ein typisches Rohdatenbild mit den beiden Kanälen. Daneben sind die beiden $\frac{1}{2}(I \pm V)$-Kanäle des korrigierten Rohbildes zu sehen. Ganz rechts ist zunächst das durch Addition der beiden Teilbilder des korrigierten Rohbildes gewonnene Spektrum des reinen Stokes- $I$-Signals zu finden. Rechts daneben das durch Subtraktion erhaltene Datenfeld des Stokes- $V$-Signals.

nach physikalischer Fragestellung lassen sich daraus eine Reihe von Informationen gewinnen, die Aufschluß über die Verhältnisse auf der Sonne geben. Einige für die vorliegende Arbeit wichtige Herangehensweisen werden im folgenden kurz besprochen.

\section{Stokes- $I$-Signal}

Als erstes und einfachstes läßt sich aus dem Stokes- $I$-Profil durch Mittlung über einen kleinen an die Absorptionslinie angrenzenden Bereich des Kontinuums Licht, das aus der unteren Photosphäre stammt, für einen Bildpunkt des beobachteten Gebietes ermitteln. Tut man dies für alle Ortspositionen entlang des Spaltes und alle Schritte eines Scans, so setzt sich daraus ein zweidimensionales Filtergramm des Kontinuumlichtes zusammen. Anhand der Kontraste der Strukturen (Granulation) eines solchen Bildes kann man z.B. grob die Qualität der Sichtbedingungen bzw. Luftruhe (engl. seeing) abschätzen.

Aus der Kernintensität oder äquivalent dazu der Einsenkung der Absorptionslinie lassen sich auf dieselbe Weise Filtergramme erzeugen. Setzt man sie ins Verhältnis zur Kontinuumsintensität, so kann man über lokale Helligkeitsschwankungen auf Temperaturunterschiede schließen. Aus der relativen Linienkernintensität läßt sich weiterhin die sogenannte Äquivalentbreite der Absorptionslinie, welches ein Maß für die insgesamt von ihr absorbierte Energie ist, errechnen. Die Äquivalentbreite ist definiert als diejenige Breite eines rechteckigen 100\%-Absorptionsstreifens, dessen Fläche gleich der Fläche der Linie ist. 

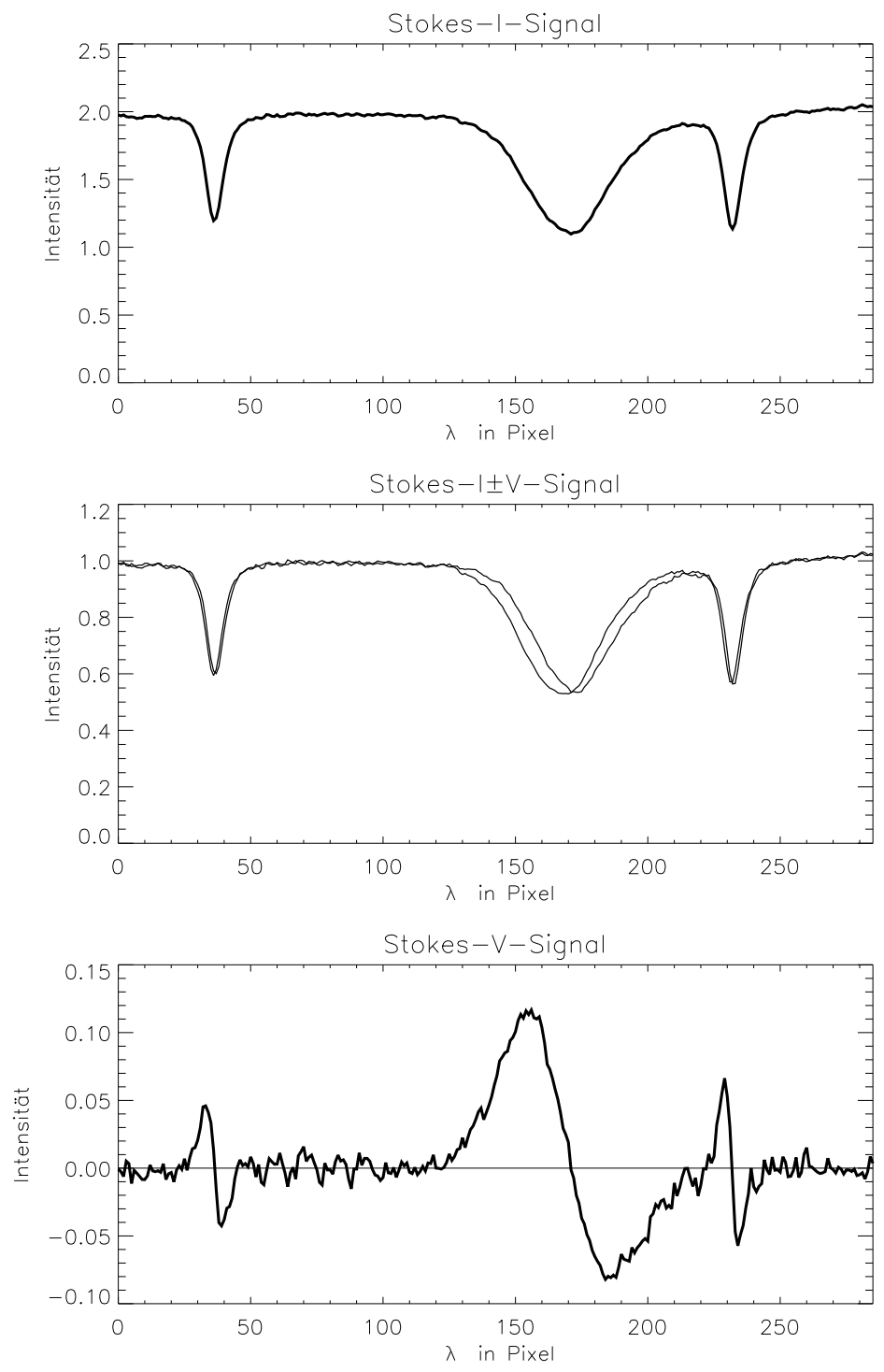

Abbildung 5.4: Dargestellt ist oben ein Spektrumsausschnitt des Stokes- $I$-Signals. Deutlich erkennbar ist eine starke Vertiefung der Fe I-Absorptionslinie, die von den beiden $\mathrm{O}_{2}$-Linien umgeben ist. Auf der x-Achse sind die 286 Positionspixel des Detektors aufgetragen. Sie stellt die spektrale Richtung dar, auf der die Wellenlänge nach rechts wächst. Auf der y-Achse ist die Intensität in relativen Einheiten aufgetragen. In dem Graphen in der Mitte sind die beiden $\frac{1}{2}(I \pm V)$-Kanäle übereinander gezeichnet aufgetragen. Deutlich erkennt man die Verschiebung der Profile der Eisenlinie gegeneinander. Unten ist das durch Subtraktion gewonnene Stokes- $V$-Signal zu sehen. Aufgrund der geringen Aufspaltung ist das $V$-Signal äußerst schwach und wird deshalb zur Bestimmung des Magnetfeldes nicht herangezogen. 
Aus den den jeweiligen Minimumpositionen der Linienprofile zugeordneten Wellenlängendifferenzen kann man mit Hilfe des Doppler-Effekts Strömungsgeschwindigkeiten am Ort der Linienentstehung berechnen. Zur genauen Linienminimumpositionsbestimmung wird eine Parabel an das Profil angepaßt.

\section{Klassischer Doppler-Effekt}

Bewegt sich solare Materie auf den Beobachter zu oder von ihm weg, so verschiebt sich, als Folge des Doppler-Effekts, eine mit dem bewegten Plasma in Verbindung stehende Spektrallinie zu kürzeren bzw. längeren Wellenlängen hin. Dieser Zusammenhang wird für Materiebewegungen, deren Geschwindigkeit $v$ sehr viel kleiner als die Lichtgeschwindigkeit $c$ ist, durch folgende Formel beschrieben:

$$
\Delta \lambda=\frac{v}{c} \cdot \lambda_{0}
$$

mit

$$
\Delta \lambda=\lambda-\lambda_{0},
$$

wobei $\lambda$ die verschobene und $\lambda_{0}$ die unverschobene Wellenlänge ist. Ist $v>0$, d.h. also eine Bewegung des Plasmas weg vom Beobachter, so erscheint die Linie rotverschoben; bei $v<0$ hingegen blauverschoben. Als Bezugswellenlänge $\lambda_{0}$ wird in der Praxis häufig die mittlere Position des Linienminimum der jeweiligen solaren Spektrallinie genommen. Das hat den Vorteil, daß man sich auf eine Größe solaren Ursprungs bezieht und damit eine Berücksichtigung der Relativbewegung zwischen Beobachter und Sonne entfällt. Man berechnet dann allerdings mit der Doppler-Formel eine relative Linienverschiebung bzw. Materiebewegung.

\section{Stokes- $V$-Signal}

Im Idealfall, d.h. bei vernachlässigbarem Übersprechen (engl. cross talk), repräsentiert das Stokes- $V$-Signal außschließlich die beiden zirkular polarisierten $\sigma$-Komponenten des longitudinalen Zeeman-Effekts. Als Crosstalk bezeichnet man die Vermischung der StokesParameter durch den optischen Aufbau. Also z.B. das Einfließen von Anteilen des Stokes$Q$ - und/oder Stokes- $U$-Signals in das Stokes- $V$-Signal durch Verdrehung der optischen Achsen der $\lambda / 4$-Platte gegen jene der Kalkspatsäulen des Polarimeters. Die Amplituden des $V$-Signals sind, im günstigen Fall geringen Crosstalk, direkt abhängig vom Winkel zwischen der Sichtrichtung des Beobachters und der Magnetfeldrichtung. Die Beträge der Amplituden haben ein Maximum bei zum Magnetfeld paralleler Blickrichtung und nehmen mit wachsendem Winkel linear mit dem Cosinus desselben ab. Asymmetrien der Stokes-Parameter lassen sich, wenn sie nicht auf Crosstalk-Effekte zurückgeführt werden können, als Gradienten eines Geschwindigkeitsfeldes interpretieren, wobei es in einigen Fällen auch noch andere Gründe haben kann. 
Aus der relativen Verschiebung des Nulldurchgangs (engl. zero crossing) des Stokes- $V$ Signals kann man wieder mit Hilfe des Doppler-Effekts Geschwindigkeiten berechnen. Die herausragenste Eigenschaft des Stokes- $V$-Signals jedoch ist die Möglichkeit, über den Abstand der Extrema des $V$-Profils die Aufspaltung der $\sigma$-Komponenten des zirkular polarisierten Lichtes zu erhalten. Die Hälfte dieses Abstandes entspricht der Wellenlängenverschiebung $\Delta \lambda$, die für die Berechnung des Betrages der magnetischen Flußdichte in die Zeeman-Formel eingesetzt werden muß. Über diesen Weg lassen sich mit reinen Intensitätsmessungen Magnetfelder auf der Sonne nachweisen.

Neben der natürlichen Linienbreite sind solare Spektrallinien noch sehr stark druckund Doppler-verbreitert. Das hat zur Folge, daß eine Aufspaltung einer solaren Absorptionslinie im sichtbaren Licht erst bei Magnetfeldern von $100 \mathrm{mT}$ bis $150 \mathrm{mT}$ deutlich wird. Schwächere Magnetfelder führen nur zu einer weiteren Linienverbreiterung. Bei der Beobachtung umgeht man zwar durch gleichzeitige Aufnahme des $(I+V)$ - und $(I-V)$ Kanals dieses Problem, bei der Datenauswertung jedoch muß man sich wieder mit diesem auseinandersetzen. So führen schwache Magnetfelder zu Stokes- $V$-Profilen, deren Signal sich kaum noch vom Hintergrundrauschen abhebt und deren Aufspaltung auch nicht mehr der Zeeman-Aufspaltung entspricht, sondern größer ist, da in diesem Fall die Verbreiterung der einzelnen Komponenten durch den Doppler-Effekt im $V$-Signal eine Rolle spielt. Da die in dieser Arbeit behandelten Magnetfelder in diesem Sinne schwach sind, muß für die polarimetrische Auswertung ein anderer Weg beschritten werden. Statt durch Differenzbildung zum Stokes- $V$-Profil zu gelangen, wertet man die $(I \pm V)$-Kanäle mittels Linienschwerpunkt-Methode (engl. center of gravity method) direkt aus.

\section{Linienschwerpunkt-Methode}

Die Linienschwerpunkt-Methode (Semel 1967) nutzt die Gegebenheit aus, daß das ISignal in den beiden $(I \pm V)$-Kanälen gleich ist und deren Unterschiedlichkeit ausschließlich auf die Überlagerung mit dem $V$-Signal zurückzuführen ist. Diese Überlagerung ruft eine Asymmetrie in der Form hervor, daß sich das Linienprofil des einen Kanals am blauen und des anderen am roten Flügel verändert; und zwar in dem Maße, wie das Magnetfeld stärker wird. Es ist somit anschaulich klar, daß mit dem sich verändernden Linienprofil auch die Flächenschwerpunkte wandern. Hier setzt die LinienschwerpunktMethode an, indem sie die Wellenlängendifferenz der Schwerpunkte der beiden Kanäle nutzt, um über den Zeeman-Effekt Magnetfelder zu messen. Dies geht im Einzelnen wie folgt:

Zuerst werden die Schwerpunkte $S\left(x_{s}, y_{s}\right)$ der Linienprofile berechnet. Da der an sich kontinuierliche Verlauf des Absorptionslinienprofils durch die $n$-Pixel einer Zeile der aufnehmenden CCD-Kamera in diskretisierter Form vorliegt, gehen die Integrale in Summen über. Die Schwerpunktkoordinaten erhält man dann mit

$$
x_{\mathrm{S}}=\frac{\sum_{i=1}^{n} x_{i} y_{i}}{\sum_{i=1}^{n} y_{i}}
$$


und

$$
y_{\mathrm{S}}=\frac{\sum_{i=1}^{n} x_{i} y_{i}}{\sum_{i=1}^{n} x_{i}}
$$

Hier stellt $i$ den Laufindex der Pixelnummer, $x_{i}$ die spektrale Position, d.h. also die Wellenlänge und $y_{i}$ die Intensität des $i$-ten Pixels, d.h. die Intensität des $(I \pm V)$-Kanals an der Stelle $x_{i}$ dar. Die $x_{\mathrm{S}}, y_{\mathrm{S}}$ sind die Schwerpunktkoordinaten des jeweils betrachteten Kanals. Zur Vereinfachung der Rechnung ist es nützlich die Absorptionsprofile um das lokale Kontinuumsniveau zu verkleinern und anschließend mit (-1) zu multiplizieren. Die gesuchte Wellenlängenverschiebung ist nun die halbe Differenz der x-Koordinaten der Linienschwerpunkte der beiden Kanäle.

$$
x_{\mathrm{S}, I+V}-x_{\mathrm{S}, I-V}=\Delta x_{\mathrm{S}}=2 \cdot \Delta \lambda_{\mathrm{S}}
$$

In gewohnter Weise wird diese Wellenlängendifferenz $\left(\Delta \lambda_{\mathrm{S}}\right)$ in die Zeeman-Formel eingesetzt. Man erhält aber statt des Betrages der magnetischen Flußdichte $|\vec{B}|$ die Sichtlinienkomponente $B_{\|}$desselben (vgl. Landi Degl' Innocenti 1992).

$$
B_{\|}=\frac{4 \pi m_{e} c}{e g_{\mathrm{eff}} \lambda_{0}^{2}} \cdot \Delta \lambda_{\mathrm{S}}
$$

Hier steht $g_{\text {eff }}$ für den effektiven Landé-Faktor, $m_{e}$ und $e$ für Masse und Ladung des Elektrons, $c$ für die Lichtgeschwindigkeit und $\lambda_{0}$ für die Ruhewellenlänge der betrachteten Linie. Durch Multiplikation der ermittelten magnetischen Flußdichte mit dem Auflösungselement $A$ geht man zu der physikalisch aussagestärkeren Größe des magnetischen Flusses $\Phi$ in Beobachtungsrichtung über:

$$
\Phi=B_{\|} \cdot A
$$

Setzt man $B_{\|}$in Tesla $\left[1 T=10^{4} \mathrm{Gau} 3\right]$ und $A$ in $\mathrm{m}^{2}$ ein, so ergibt sich für $\Phi$ die SIEinheit Weber [Wb].

Die Methode ist unabhängig vom Aufspaltungsmuster (Rees und Semel 1979) und liefert mit einer Genauigkeit von ca. 10\% Ergebnisse für jede Inklination des magnetischen Feldvektors. Sie hat den Vorteil, nicht auf Amplitudensättigung zu reagieren und ist weitgehend unempfindlich gegen instrumentelle Polarisation bzw. Crosstalk (del Toro Iniesta et al. 1990).

Hat man erst einmal die Schwerpunktkoordinaten, so lassen sich aus der Verschiebung der mittleren Wellenlängen, gebildet aus der halben Summe der $x$-Koordinaten der Linienschwerpunkte der beiden Kanäle, Doppler-Geschwindigkeiten errechnen. Auch lassen sich mit den $y$-Koordinaten der Schwerpunkte Aussagen über die Intensitäts-Asymmetrie $\delta y_{\mathrm{S}}$ der Profile machen:

$$
\delta y_{\mathrm{S}}=\frac{y_{\mathrm{S}, \text { blau }}-y_{\mathrm{S}, \text { rot }}}{y_{\mathrm{S}, \text { blau }}+y_{\mathrm{S}, \text { rot }}}
$$




\subsection{Daten vom Vakuum-Turm-Teleskop}

Zur Beobachtung der Chromosphäre eignen sich besonders gut starke Fraunhofer-Linien, da im Zentrum einer solchen Linie das Absorptionsvermögen der Sonnenmaterie so groß ist, daß die Restintensität der Strahlung nur noch aus höchsten, bereits in der Chromosphäre liegenden Schichten austreten kann. Da dieses Verhalten besonders auffällig bei der $K$-Linie (ca. 393,4 nm) des einfach ionisierten Kalziums (Ca II) in Erscheinung tritt, wurden zum Verfolgen der Ereignisse in der Chromosphäre monochromatische Aufnahmen in dieser Linie angefertigt. In der Ca II-Linie taucht die Emission der Chromosphäre aus dem Kern der Absorptionslinie heraus, wobei in ihrer Mitte die optische Dicke sogar so groß wird, daß auch die chromosphärische Emission wieder eine Selbstabsorption zeigt. Das liegt daran, daß die Quellfunktion dieser Linie zu höheren Schichten abnimmt, obwohl die Temperatur nach außen im Mittel zunimmt. Das Intensitätsminimum zu den Linienflanken bezeichnet man mit $K_{1}$, die Emission im Kerngebiet mit $K_{2}$ und die sich im Linienzentrum abspielende sogenannte Selbstumkehr mit $K_{3}$. Weiterhin bezeichnen die Indizes $v$ und $r$ den "violetten" bzw. "roten" Linienflügel. Im vorliegenden Fall wurde mit einem Lyot-Filter die $K_{2 v}$ Emissionsspitze nahe des Ca II-Linienkerns bei 393,3 nm mit einer Halbwertbreite von 0,06 nm aufgenommen. Die Daten wurden mit einer 1024 Pixel $\times 1024$ Pixel CCD-Kamera aufgenommen, wobei der Pixelabstand um einen Faktor 2 durch Addition benachbarter Pixelintensitäten verkleinert wurde. Bei einem Auflösungsvermögen von 0,366 $\frac{1}{\text { Pixel }}$ und Abschneiden eines schmalen Randes ergibt sich ein Feld von ca. 187" × 184". Die zeitliche Kadenz der Beobachtungen betrug 30s bei einer Integrationszeit von $0,25 \mathrm{~s}$. Im Rahmen des Beobachtungstages entstanden 800 Filtergramme der chromosphärischen Linie.

\subsubsection{Datenbearbeitung}

Auch hier wird zunächst aus einer Reihe von Dunkelbildern das Bild des mittleren Dunkelstroms berechnet. Eine Reinigung der Filtergramme von Staub, Kratzern und sonstigen Verunreinigungen und Störsignalen fällt hier jedoch einfacher als beim GCT aus. Die dazu nötigen Korrekturbilder, die sogenannten Flatfields, wurden aufgenommen, während die Spiegel des Teleskops schnell und gleichmäßig bewegt Licht von ruhigen Gebieten der Sonnenoberfläche einfingen. Die auf diese Weise entstandenen Korrekturbilder zeigen, außer den störenden Verdunklungen des auf den optischen Oberflächen liegenden Staubs und anderen Störungen, keinerlei Objektinformation. Die zur physikalischen Auswertung bereiten Filtergramme $I(i, j)$ entstehen durch Division von, um das gemittelte Dunkelbild $D(i, j)$ reduzierte Rohbild $\tilde{I}(i, j)$, durch, um das gemittelte Dunkelbild reduzierte mittlere Flatfield $F(i, j) ; i, j$ stellen die Zeilen und Spaltenindizes des CCD-Chips dar.

$$
I(i, j)=\frac{\tilde{I}(i, j)-D(i, j)}{F(i, j)-D(i, j)}
$$




\subsection{Daten von SUMER}

Das Beobachtungsprogramm von SUMER begann um 7:56 UT mit der Aufzeichnung eines Kontextbildes in der chromosphärischen He I-Linie bei 58,4 nm, deren Entstehungstemperatur $\sim 3 \cdot 10^{4} \mathrm{~K}$ beträgt. Hierzu wurde ein Spalt mit einer Länge entsprechend 300" und einer Breite entsprechend 1" verwendet. Wie bei SUMER üblich, war der Spalt parallel zur Nord-Süd-Richtung der Sonne orientiert. Diese Richtung entspricht der Sonnenrotationsachse. Im Hinblick auf eine vollständige Abdeckung wurde die Schrittweite mit 1,"125 der Spaltbreite angepaßt. Die Schrittweite ergibt sich aus drei SUMERElementarschritten von je 0," 375. Das Kontextbild besteht aus einem Scan von 71 Schritten, wobei an jeder Schrittposition ein Spektrum der Emissionslinie vom Detektor A aufgezeichnet wurde. Nach jeweils 15 s Belichtungszeit wurde eine 50 Pixel $\times 360$ Pixel große Fläche des Detektors ausgelesen und abgespeichert. Nach der Datenreduktion ergeben sich für die Ausdehnung eines Filtergramms einschließlich unbelichteter Randbereiche aus den Spektren der neutralen Heliumlinie in Ost-West-Richtung (= Schrittrichtung) ca. 80" und für die Spaltrichtung etwa 361", da der Detektor A bei 58,4 nm eine Auflösung von 1, $003 \frac{\text { " }}{\text { Pixel }}$ hat. Es ist hier zu beachten, daß zwar von der Erde aus gesehen ein Winkel von $1^{\prime \prime} \sim 725 \mathrm{~km}$ auf der Sonne entspricht, jedoch vom näher an der Sonne gelegenen L1 aus schon $\sim 715 \mathrm{~km}$ unter einer Bogensekunde erscheinen. Berücksichtigt man dies, ergibt sich für das Helium-Bild eine Größe von $79^{\prime \prime} \times 356^{\prime \prime}$.

Anschließend wurde der Spalt auf die zentrale Position des Kontextbildes gefahren und dort die nächsten knapp drei Stunden, von 8:16 UT bis 11:06 UT, rotationskompensiert gehalten. Das Folgen der Sonnenrotation wurde realisiert, indem das Bild alle 145,7s um $0, " 375$ nach Osten bewegt wurde. Dadurch hat man erreicht, daß der Spalt immer dieselbe Region der Sonne gesehen hat. In diesem Zeitraum (10146s) wurden alle 36s drei Spektrumsausschnitte mit unterschiedlichen Emissionslinien in derselben Weise, wie vorher schon bei der Heliumlinie, aufgenommen. Die Belichtungszeit war, bedingt durch die Telemetrie, mit jeweils $35 \mathrm{~s}$ allerdings länger als die zuvor bei der He I-Linie gewählte. Die kühlste der drei beobachteten Linien ist die chromosphärische C I-Linie bei 156,1 nm. Diese in Emission erscheinende Linie des neutralen Kohlenstoffs hat eine Entstehungstemperatur von $\sim 1 \cdot 10^{4} \mathrm{~K}$. Die nächste Linie ist die der Übergangsregion entstammende dreifach ionisierte C IV-Linie bei 154,82 nm. Sie hat eine Entstehungstemperatur von $\sim 1 \cdot 10^{5} \mathrm{~K}$. Als drittes und letztes wurde die siebenfach ionisierte Neonlinie (Ne VIII) bei $77,04 \mathrm{~nm}$ aufgenommen. Dies ist eine Linie, die bei $\sim 8 \cdot 10^{5} \mathrm{~K}$ aus der unteren Korona emittiert wird. Beispiele der aufgenommenen Spektren finden sich in Abb. 5.5. In den Abb. 5.6, 5.7, 5.8 und 5.9 sind die gemittelten Spektrumsausschnitte dargestellt. Die Identifizierung der abgebildeten Emissionslinien ist anhand des SUMER Spektral-Atlas von Curdt et al. (2001) vorgenommen worden. Da SUMER Linien erster und zweiter Ordnung superponiert abbildet, wurden die in zweiter Ordnung aufgenommenen He Iund Ne VIII-Spektren mit dem unbeschichteten Teil des Detektors (bare MCP) beobachtet, um störende Linien erster Ordnung zu unterdrücken. Die Entstehungstemperaturen der beobachteten Emissions-Linien wurden mit Hilfe von Feldman et al. (2000) ermittelt. 


\subsubsection{Datenbearbeitung}

Die Datenverarbeitung der SUMER-Spektren erfolgte gemäß der hierfür üblichen Verfahrensweise. Um eine höhere Datenübertragungsgeschwindigkeit zu erreichen, wurden die Daten der Beobachtung an Bord von SOHO komprimiert. Deshalb müssen sie in einem ersten Arbeitsschritt entpackt werden. In regelmäßigen Abständen von etwa einem Monat werden Flatfield-Matrizen erzeugt, um Veränderungen, verursacht durch Alterung und Beschädigung, zu erkennen und in der Datenauswertung berücksichtigen zu können. Zur bestmöglichen Korrektur wurden deshalb diejenigen Flatfields aus dem SUMER-Archiv gesucht und auf die Daten angewandt, die zeitlich den geringsten Abstand zur Beobachtungskampagne hatten. Da die Halbleiterkameras von SUMER eine Verzeichnung der aufgenommenen Spektren bewirken, werden in einem dritten Arbeitsschritt diese geometrischen Verzeichnungen behoben. Weitere Korrekturschritte waren nicht erforderlich.

\subsubsection{Bestimmung physikalischer Größen}

Aus den He I-Spektren wurden durch Integration in Dispersionsrichtung eindimensionale Vektoren erzeugt. Die 71 entstandenen Vektoren wurden gemäß der Scanrichtung aneinander gesetzt und die unterschiedlichen Maßstäbe von Ost-West- und Nord-Süd-Achse ausgeglichen. So entstand ein Filtergramm im Lichte von Helium. Auf diese Weise kann man auch die jeweils 284 Spektren der drei Emissionslinien aneinander setzen. Man erhält dann jedoch kein Bild mit zwei Ortsdimensionen, sondern ein sogenanntes xtDiagramm. Das ist ein Bild mit einer Orts- und einer Zeitdimension, d.h. eine Art Film mit 1-dimensionalen Bildern. Man kann anhand dieser Auftragung ein Gebiet von 1" Breite mal 342" in seiner zeilichen Entwicklung verfolgen, und zwar, je nach gewählter Spektrallinie, in drei verschiedenen Höhen in der Sonnenatmosphäre. Als nächster Auswertungsschritt wurde eine Gaußkurve an die Linienprofile der Spektren der drei solaren Emissionslinien angepaßt. Die Anpassung der Gaußkurve

$$
f(x)=A_{0} \cdot e^{-\frac{\left(x-A_{1}\right)^{2}}{2 A_{2}^{2}}}
$$

an die Linienprofile geschieht mit den Parametern $A_{0}, A_{1}$ und $A_{2}$. Der Parameter $A_{0}$ stellt die Maximalamplitude der Kurve respektive die Maximalintensität des spektralen Verlaufs der Linien dar. $A_{0}$ ist also die Linienzentrumsintensität. $A_{1}$ gibt die Zentralposition der Gaußkurve bzw. des Profils an. Es ist möglich, über die relativen Verschiebungen der Zentralpositionen zum Mittelwert, mit Hilfe des Doppler-Effekts relative Geschwindigkeiten der strahlenden Materie zu ermitteln. Mit $A_{2}$ wird die halbe Breite der Emissionslinie bei einer Höhe bzw. Intensität von $e^{-1 / 2}(\approx 0,61)$ mal der Kernintensität $A_{0}$ angegeben. Multipliziert man $A_{2}$ mit $2 \cdot \sqrt{2 \cdot \ln (2)}$, so erhält man die Halbwertsbreite der Linie. 

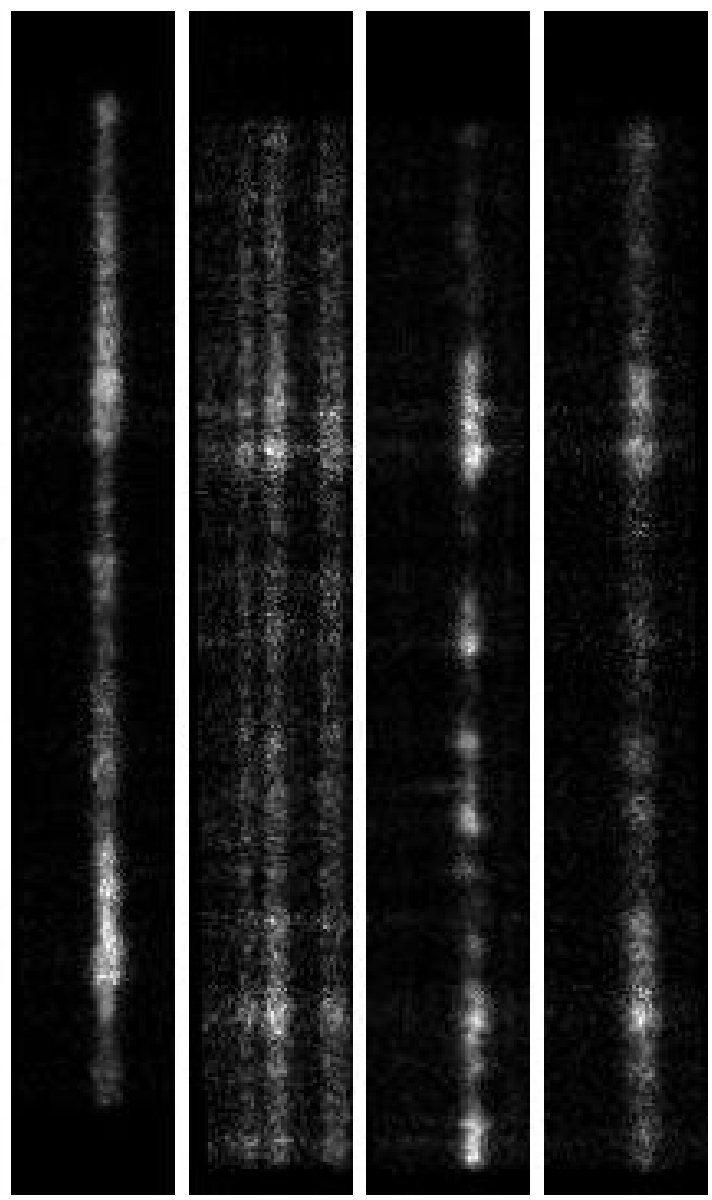

Abbildung 5.5: Spektrumsausschnitt mit Emissionslinien von SUMER. Von links nach rechts sieht man zunächst die EUV-Linie des neutralen Heliums (He I) bei $58,4 \mathrm{~nm}$. Sie entsteht bei $2,5 \cdot 10^{4} \mathrm{~K}$ bis $5,5 \cdot 10^{4} \mathrm{~K}$ in der Chromospäre. Daneben der Spektrumsausschnitt, der für die Linien des neutralen Kohlenstoffs (C I) bei 156,1 nm aufgenommen wurde (vgl. auch Abb. 5.6). Auch sie entsteht in der Chromospäre. Als nächstes folgt die in der Übergangsregion bei $0,8 \cdot 10^{5} \mathrm{~K}$ bis $1,5 \cdot 10^{5} \mathrm{~K}$ entstehende $\mathrm{C}$ IV-Linie mit einer Wellenlänge von 154,8 nm. Den Abschluß bildet die 4, $9 \cdot 10^{5} \mathrm{~K}$ bis $8,9 \cdot 10^{5} \mathrm{~K}$ heiße koronale Ne vin-Linie bei $77,0 \mathrm{~nm}$. Die Datenfelder haben jeweils die Größe von 50 Pixeln in spektraler und 360 Pixeln in räumlicher Richtung. Da sich sowohl die räumliche Ausdehnung (wegen der Verzeichnung durch die Spektrographenabbildung) als auch spektrale Dispersion mit der Wellenlänge ändern, entsprechen die 360 Pixel in Ortsrichtung 356" (von der Erde aus) im Helium, ca. 342" bei Ne ViII und C IV und 341" im Lichte des neutralen Kohlenstoffs. In den Abbildungen der He I- und Ne VIII-Linie entsprechen die 50 Pixel in spektraler Richtung etwa $0,1 \mathrm{~nm}$ (Beobachtung in zweiter Ordnung). Die abgebildeten Wellenlängenintervalle in der Umgebung der C I- und C IV-Linie haben eine Ausdehnung von etwa 0,2 nm (Beobachtung in erster Ordnung). 


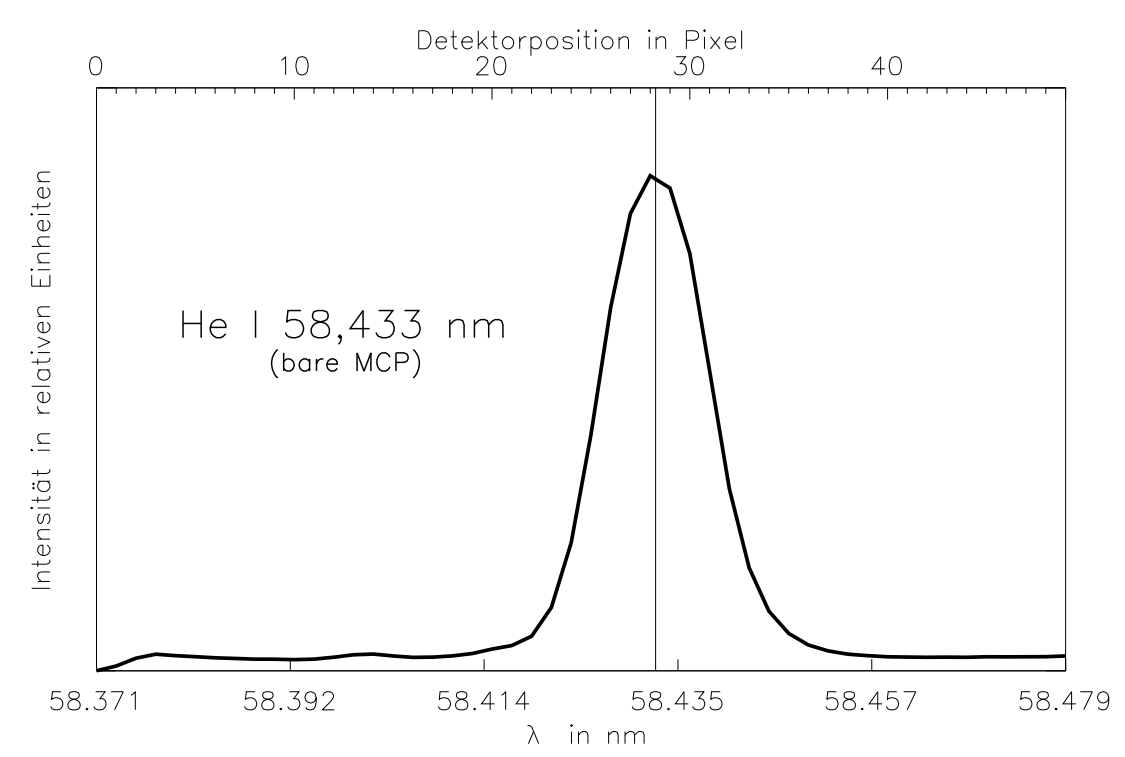

Abbildung 5.6: Gemittelter Spektrumsausschnitt der He I-Linie.

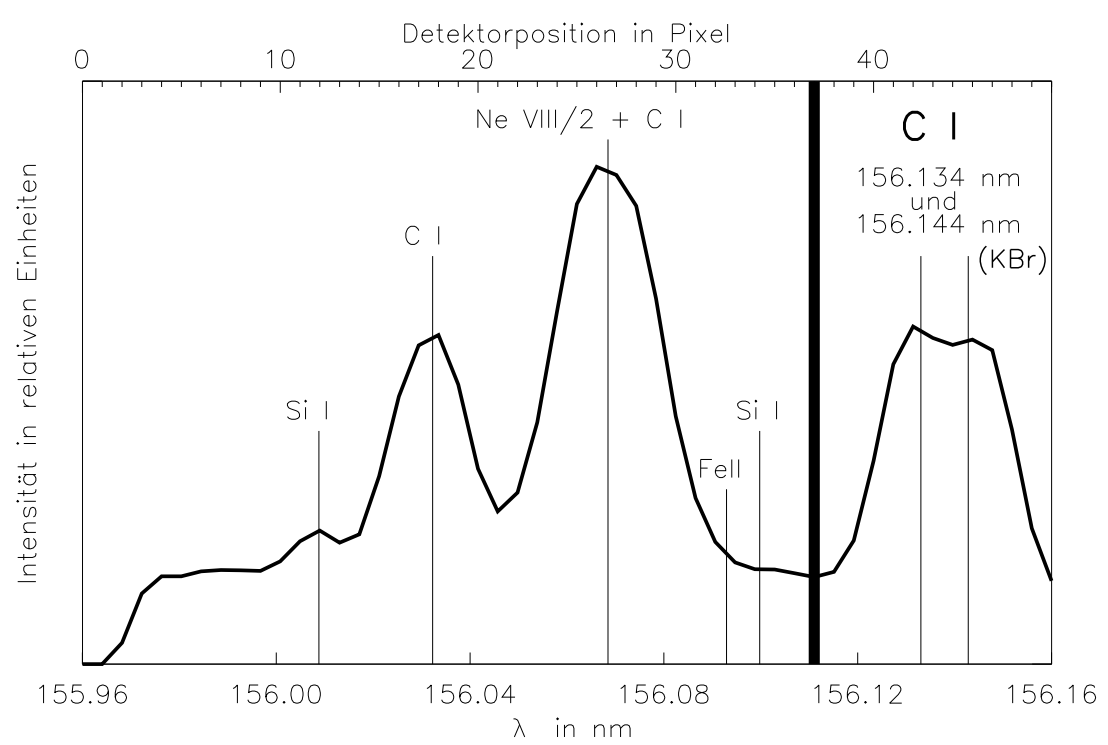

Abbildung 5.7: Gemittelter Spektrumsausschnitt der C I-Linien. Für die weitere Auswertung wurde nur der Spektrumsausschnitt rechts von der dicken Markierungslinie betrachtet. 


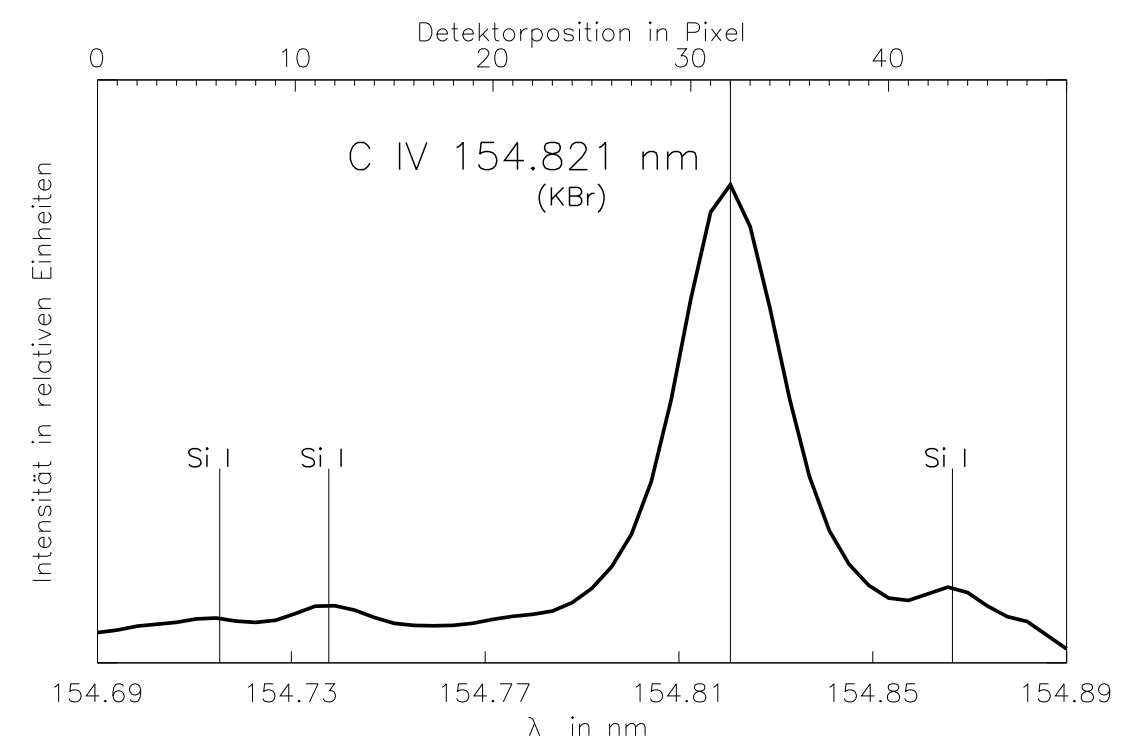

Abbildung 5.8: Gemittelter Spektrumsausschnitt der C IV-Linie.

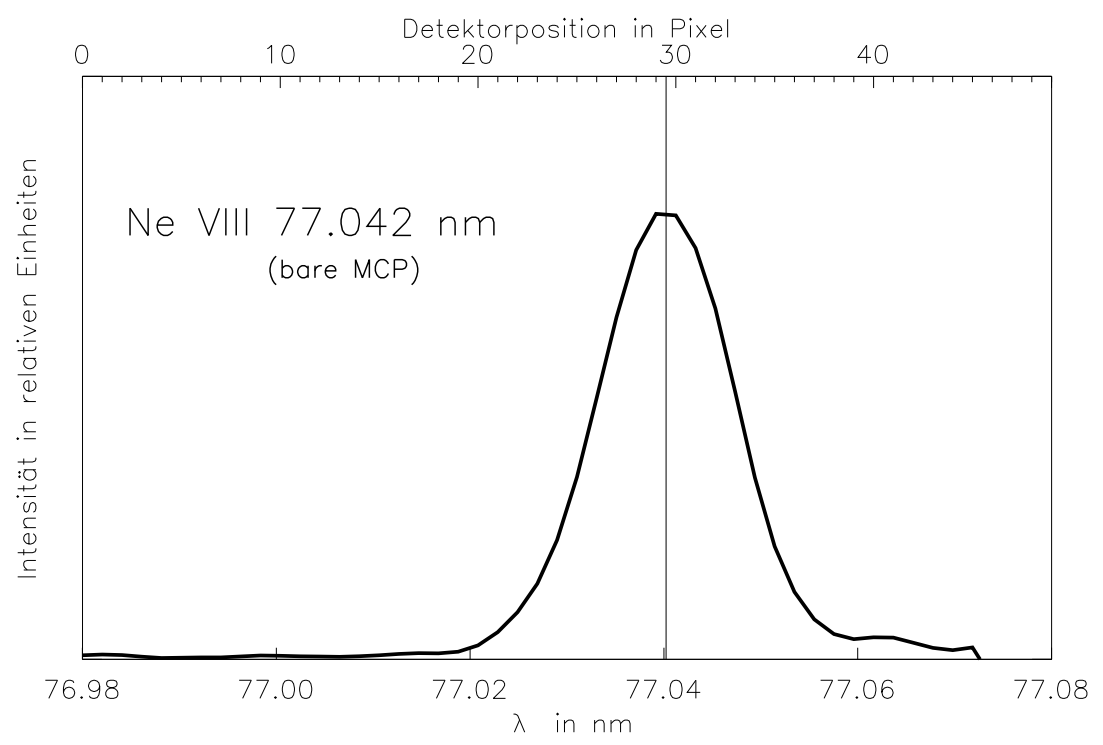

Abbildung 5.9: Gemittelter Spektrumsausschnitt der Ne viII-Linie. 


\section{Koordination der Beobachtung}

Die größte Herausforderung dieser Beobachtungskampagne stellte die exakte Koordination der beteiligten Teleskope dar. Da jedes Teleskop für sich die Mitte der Sonnenscheibe bestimmt und im folgenden als Bezugspunkt für alle weiteren Einstellungen verwendet, ist es möglich, daß ein Unterschied der Sichtrichtungen (engl. pointing) untereinander entsteht. Die beiden, sehr nahe beieinander stehenden, erdgebundenen Teleskope VTT und GCT wurden an einer kleinen, dunklen, solaren Pore aufeinander abgestimmt. Um mit SUMER möglichst synchron zu arbeiten, wurden an allen Teleskopen SOHO-Koordinaten benutzt und im GCT das Sonnenbild so gedreht, daß wie bei SUMER die Projektion der Rotationsachse der Sonne parallel zum Spektrographenspalt lag. Darüberhinaus wurde ein Gebiet in der Sonnenscheibenmitte gewählt, was nicht nur sehr vorteilhaft für die Bearbeitung der Magnetfelddaten mit der Linienschwerpunktmethode ist, sondern sich auch sehr zuverlässig einstellen läßt. Das gemeinsame Pointing wurde durch die Tatsache erschwert, daß das SUMER steuernde Beobachtungsprogramm (UDP $=$ User Defined Program) am Tag vor jeder Beobachtung fertiggestellt, getestet und zur Raumsonde gesendet werden mußte. Daher war es weder möglich, kurzfristig das Beobachtungsgebiet zu wechseln, noch bei laufender Beobachtung das Pointing aufeinander abzustimmen. Darüber hinaus unterlag SUMER aufgrund technischer Probleme starken Restriktionen. So durfte nur ein Scan als Überblicksbild am Anfang jedes Beobachtungstages gemacht und danach nur noch rotationskompensierte Spektren aufgenommen werden, wodurch das Gebiet auf einen Ausschnitt von $1^{\prime \prime} \times 300^{\prime \prime}$ schrumpfte. Weiterhin unterlag der Detektor einer starken Begrenzung der Zählraten (engl. counts) pro Pixel und Sekunde, so daß nur äußerst schwach aktive Gebiete für die Kampagne in Frage kamen. Am GCT konnte die Fläche der einzelnen Magnetogramme nicht größer als $60^{\prime \prime} \times 57^{\prime \prime}$ gewählt werden, da sonst die Wiederholrate und damit die zeitliche Auflösung der Beobachtung zu gering geworden wäre. Aus alledem resultierte, daß ein äußerst schwach strukturiertes Gebiet gleichzeitig präzise aufgefunden werden mußte. Neben den Kontextbildern sollten, als weitere Hilfe für die später bei der Auswertung wichtige exakte Überdeckung (engl. alignment) der Aufnahmen der verschiedenen Teleskope miteinander, ständig großformatige Ca-Filtergramme aufgenommen werden. Dies gelang am 10.05.1999 jedoch nicht in der beabsichtigten Perfektion, da kurz nach Beginn der Aufnahmen der Kameraverschluß nicht mehr zuverlässig funktionierte. 


\section{SOHO-Koordinatensystem}

Die Rotationsachse der Sonne ist gegen die Normale zur Ebene der Ekliptik um den Winkel $\mathrm{i}=7^{\circ} 15^{\prime}$ geneigt; von der Erde bzw. SOHO aus gesehen ändert sich daher ihre Lage im Laufe eines Jahres. Um bei der koordinierten Beobachtung ein einheitliches Orientierungssystem zu haben, wurde auch an den erdgebundenen Teleskopen das sogenannte SOHO-Koordinatensystem verwendet. Es ist ein kartesisches Koordinatensystem, dessen Ursprung in der Scheibenmitte liegt und dessen Ordinate auf die Rotationsachse der Sonne projiziert wird. Betrachtet man die Sonne von der Erde bzw. SOHO aus, so erscheint sie als Scheibe. Man denkt sich nun eine Gerade parallel zur Rotationsachse der Sonne, die durch das Zentrum verläuft und so die Scheibe in zwei Flächen gleicher Größe teilt. Der obere Schnittpunkt der Geraden mit dem Rand der Scheibe wird "Norden", der untere "Süden" genannt. Man bezeichnet weiterhin die linke Seite als "Osten" und die rechte als "Westen". Nach dieser Festlegung der "Himmelsrichtungen" bezeichnet das SOHO-Koordinatensystem die Richtung vom Ursprung (Scheibenmitte) nach Westen als positive $\mathrm{x}$-Richtung und diejenige nach Norden als positive y-Richtung. Die Achsen dieses Systems sind in Einheiten von einer Bogensekunde geteilt. Von der Erde aus gesehen überstreicht der Winkel von $1^{\prime \prime}$ im Mittel $725 \mathrm{~km}$ auf der Sonne, von der näher an der Sonne plazierten Sonde jedoch nur ca. $715 \mathrm{~km}$. 


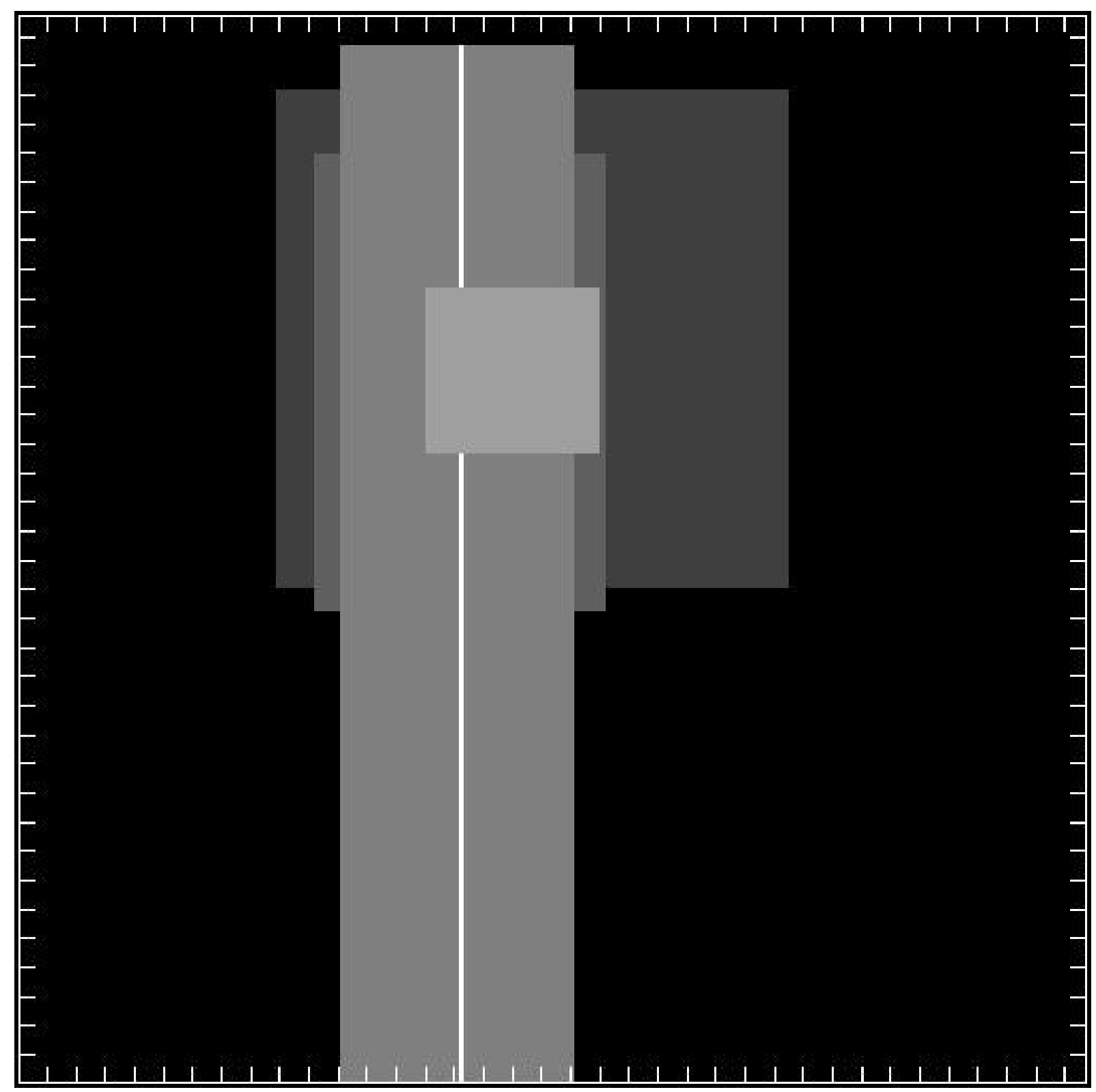

Abbildung 6.1: Auf diesem Bild ist die Überdeckung der von den verschiedenen Teleskopen aufgenommenen Datenfelder qualitativ dargestellt. Zuunterst liegt im dunkelsten grau das fast quadratische VTT $C a$-Filtergramm $\left(176^{\prime \prime} \times 171^{\prime \prime}\right)$ auf dem schwarzen Hintergrund. Darüber das $100^{\prime \prime} \times 157^{\prime \prime}$ GCT-Kontextbild. Dann das sehr lange und schmale He I-Bild von SUMER $\left(79^{\prime \prime} \times 356^{\prime \prime}\right)$ mit der durch einen hellen vertikalen Strich gekennzeichneten Position, an der die C I-, C IV- und Ne viII-Spektren aufgenommen wurden. Obenauf ist in hellgrau die Position der $60^{\prime \prime} \times 57^{\prime \prime}$ großen GCT-Datenfelder eingezeichnet. Der Abstand der Markierungsstriche (engl. tick marks) beträgt 10 Bogensekunden. 


\section{Auswertung der Übersichtsbilder}

Die Übersichtsbilder wurden von jedem der beteiligten Teleskope zu Beginn eines Beobachtungstages mehr oder weniger gleichzeitig aufgenommen. Diese Bilder wurden aus zwei Gründen erstellt:

Erstens wurde mit ihrer Hilfe die Orientierung und Überlappung der Bilder bzw. Spektren der verschiedenen Teleskope untereinander aufgefunden.

Zweitens ermöglichten sie die Einordnung des Beobachtungsobjektes in die großskalige Struktur der Umgebung.

Für das Kontextbild des GCTs wurden am 10.5.1999 an 600 Positionen Spektren aufgenommen. Diese Serie von Fe I-Spektren dauerte 11 Minuten von 7:56 UT bis ca. 8:07 UT. Aus den gewonnenen Daten konnte das in Abbildung 7.1 links gezeigte $100^{\prime \prime} \times 157^{\prime \prime}$ große Linienzentrumsintensitätsbild rekonstruiert werden. Es ist so orientiert, daß oben Norden und links Osten ist. Die Mitte des Bildes liegt bei $x=-20^{\prime \prime}$ und $y=-10^{\prime \prime}$, also fast genau in Sonnenscheibenmitte. Das Bild besteht aus 273 Pixel $\times 429$ Pixel, bei einem Abbildungsmaßstab von 0,366 $\frac{11}{\text { Pixel }}$. Auf diesen von den VTT-Daten herrührenden Maßstab wurden, um der Vergleichbarkeit willen, alle in die Auswertung eingegangenen Daten gebracht. Deutlich heben sich eine ganze Reihe von kleinen, sehr hellen Gebieten vom grau-schwarzen Hintergrund ab. Um die Intensitäten der Aufhellungen besser beurteilen zu können, wurden die Intensitätswerte des Linienzentrumsintensitätsbildes (Abb. 7.1 links) in relativen Einheiten entlang der Ost-West-Achse gegen die Position in Pixeln zeilenweise aufgetragen (Abb. 7.2 links). Während der grau-schwarze Hintergrund bei Werten zwischen 0,98 und 1,00 liegt, erreichen die Aufhellungen Werte bis über 1,3. In Abb. 7.1 rechts sieht man das aus den Spektren des Kontextbildes gewonnene Magnetogramm. Die weißen Bereiche entsprechen positiver Polarität (magnetisch Norden). Sie erreichen bei einem Aufösungselement von $\left(0,{ }^{\prime \prime} 77\right)^{2}$ einen magnetischen Fluß von bis zu $6,6 \cdot 10^{9} \mathrm{~Wb}$ (entsprechend einer mittleren Flußdichte von 212 Gauß $=21,2 \mathrm{mT}$ ). In den schwarzen Gebieten negativer Polarität (magnetisch Süden) erreicht der magnetische Fluß bis $\mathrm{zu}-4,1 \cdot 10^{9} \mathrm{~Wb}$ (entsprechend einer mittleren Flußdichte von $132 \mathrm{Gau}=13,2 \mathrm{mT}$ ). Um die Schwankungen des magnetischen Flusses zu verdeutlichen, wurde in Abb. 7.2 rechts der magnetische Fluß in Wb gegen die Ortsposition in Bogensekunden entlang der Ost-West-Achse zeilenweise aufgetragen. In Abb. 7.3 links ist noch einmal das Magnetogramm abgebildet; Hier sind jedoch, zur Verdeutlichung der magnetischen Struktur, Isokonturlinien bei $\pm 3,0 \cdot 10^{8} \mathrm{~Wb}, \pm 5,0 \cdot 10^{8} \mathrm{~Wb}$, 


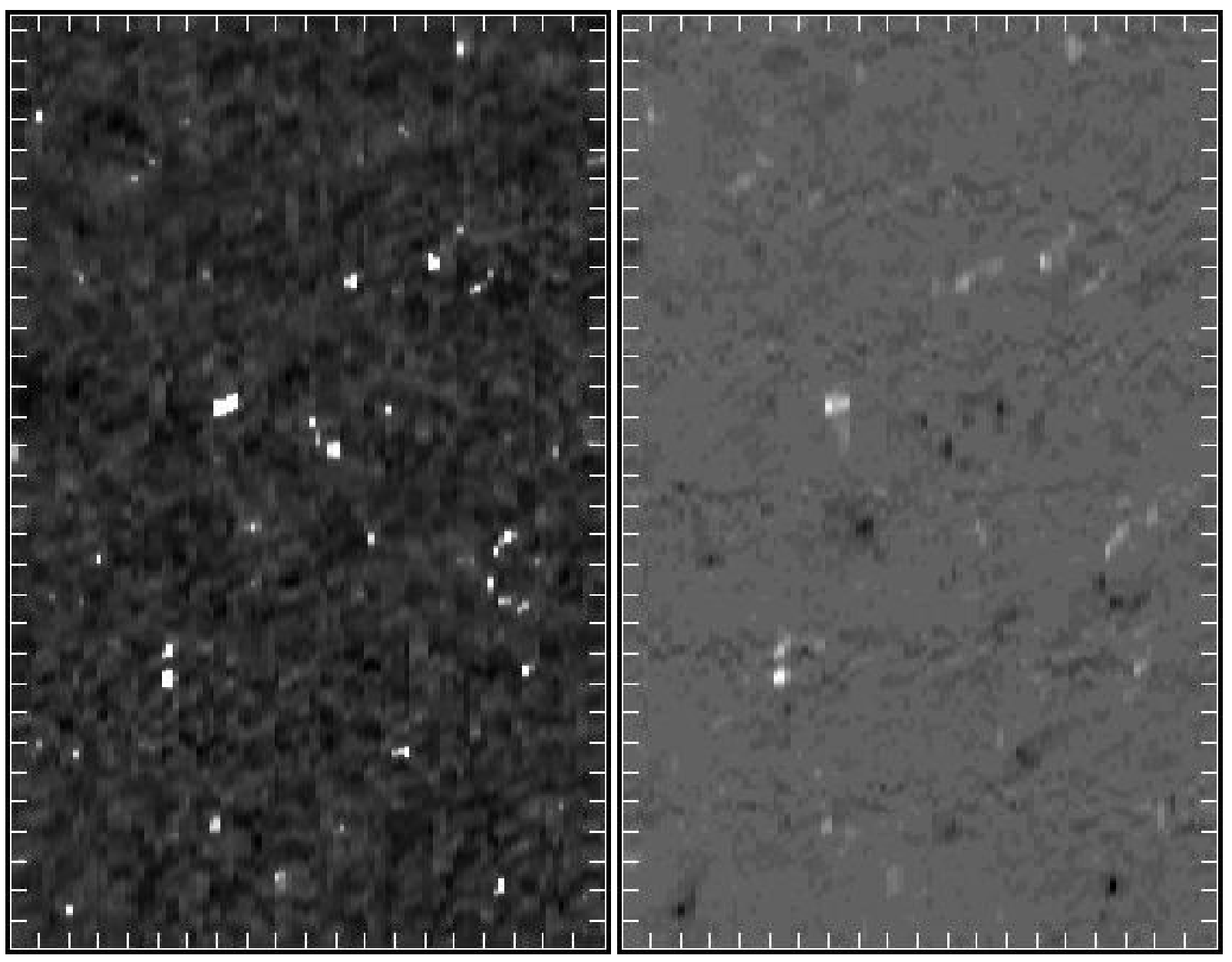

Abbildung 7.1: links: 100" $\times 157^{\prime \prime}$ (273 Pixel mal 429 Pixel) großes GCT-Kontextbild im Lichte des Fe I-Linienzentrums bei 630,25 nm. Es ist zusammengesetzt aus 6 Scans von jeweils 100 Spektren. Das Aufnehmen aller 600 Spektren dauerte 11 Minuten, von 7:56 UT bis ca. 8:07 UT. Oben ist Norden und links Osten. Die Mitte des Bildes liegt bei $x=-20^{\prime \prime}$ und $y=-10^{\prime \prime}$. Deutlich heben sich eine ganze Reihe von kleinen sehr hellen Gebieten vom Hintergrund ab. rechts: Das aus den Spektren des Kontextbildes gewonnene Magnetogramm. Die weißen Bereiche entsprechen positiver Polarität. Sie erreichen bei einem Auflösungselement von $\left(0,{ }^{\prime \prime} 77\right)^{2}$ einen magnetischen Fluß von bis $\mathrm{zu} 6,6 \cdot 10^{9} \mathrm{~Wb}$ (entsprechend einer mittleren Flußdichte von $212 \mathrm{Gau}=21,2 \mathrm{mT}$ ). In den schwarzen Gebieten negativer Polarität erreicht der magnetische Fluß bis zu $-4,1 \cdot 10^{9} \mathrm{~Wb}$ (entsprechend einer mittleren Flußdichte von 132 Gauß=13,2 mT). Der Abstand zwischen den Markierungen beträgt in beiden Bildern fünf Bogensekunden. 

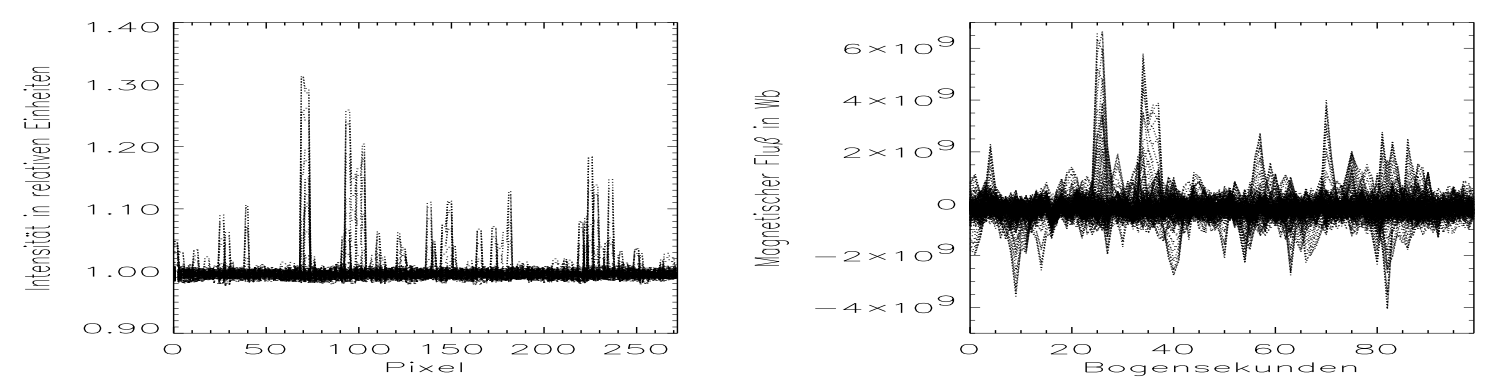

Abbildung 7.2: links: Das Linienzentrumsintesitätsbild in zeilenweiser Auftragung. rechts: Das Magnetogramm in zeilenweiser Auftragung. Ein Pixel entspricht 0,366 Bogensekunden.

$\pm 7,5 \cdot 10^{8} \mathrm{~Wb}$ und $\pm 1,0 \cdot 10^{9} \mathrm{~Wb}$ eingezeichnet. Die vertikale weiße Linie markiert die Position und Breite des SUMER-Spaltes, während das mit dünnen weißen Linien gezeichnete Rechteck die Startposition der GCT-Bilder der Beobachtungsserie von $60^{\prime \prime} \times 57^{\prime \prime}$ wiedergibt. Rechts daneben findet sich eine stark vereinfachte Version des Linienzentrumsbildes. Hier wurde der Hintergrund, der besseren Übersicht halber, gleichmäßig schwarz und alle Aufhellungen ab 10\% des Maximalkontrastes weiß gezeichnet. Neben den schon aus Abb. 7.3 links bekannten Positionsmarkierungen wurden zusätzlich die Isokonturlinien des Magnetogramms bei $\pm 3,0 \cdot 10^{8} \mathrm{~Wb}$ übernommen. Man erkennt, daß fast alle Aufhellungen des Intensitätsbildes mit magnetischer Aktivität einhergehen.

Die Ca II $K$-Filtergramme des VTTs in Abb. 7.4 (links ein zeitlich gemitteltes, daneben ein Einzelbild) mußten für die weitere Auswertung zunächst um $20{ }^{\circ} 4 \mathrm{im}$ Uhrzeigersinn gedreht und dann an der Nord-Süd-Achse (vgl. in Abb. 7.4 eingezeichnetes Richtungskreuz) gespiegelt werden. Besonders deutlich tritt die großräumige zellartige Struktur der Supergranulation in dem gemittelten Bild hervor (Abb. 7.4 links). Abb. 7.5 links zeigt das, in die "richtige" Orientierung gebrachte, erste Ca II $K$-Filtergramm der Tagesserie. Es wurde, mit einer Belichtungszeit von 0,25 s, um 8:18 UT aufgenommen, hat also einen zeitlichen Abstand von $\sim \frac{1}{4} \mathrm{~h}$ zum "zeitlichen Schwerpunkt" des GCT-Kontextbildes. Neben diesem Bild befindet sich nochmal das gleiche Bild, jedoch heben hier Isokonturlinien die supergranulare Struktur deutlich hervor. Die Isokonturlinien befinden sich bei 30\%, 50\% und 70\% des Maximalkontrastes. Weiterhin sind wieder die Positionen des SUMER-Spaltes und der GCT-Startposition markiert. In diesem, mit weißen Linien eingezeichneten, Rechteck befindet sich eine annähernd elliptische supergranulare Zelle. Sie

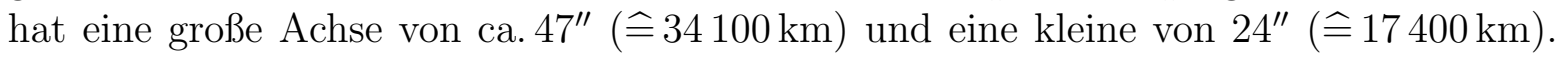
Deutlich heben sich eine ganze Reihe von kleineren Aufhellungen, die fast alle in der Nähe oder auf dem Rand der Supergranule liegen, vom Untergrund ab. Unterlegt man dem Isokonturlinienanteil des Bildes (Abb. 7.5 rechts) das GCT-Magnetogramm (Abb. 7.1 rechts), so erkennt man, daß auf den Rändern der eben erwähnten Supergranule die Gebiete magnetischer Aktivität liegen (vgl. Abb. 7.6). Bedenkt man, daß zwischen dem GCT-Kontextbild und dem Ca-Filtergramm des VTTs eine zeitliche Differenz von ca. 15 


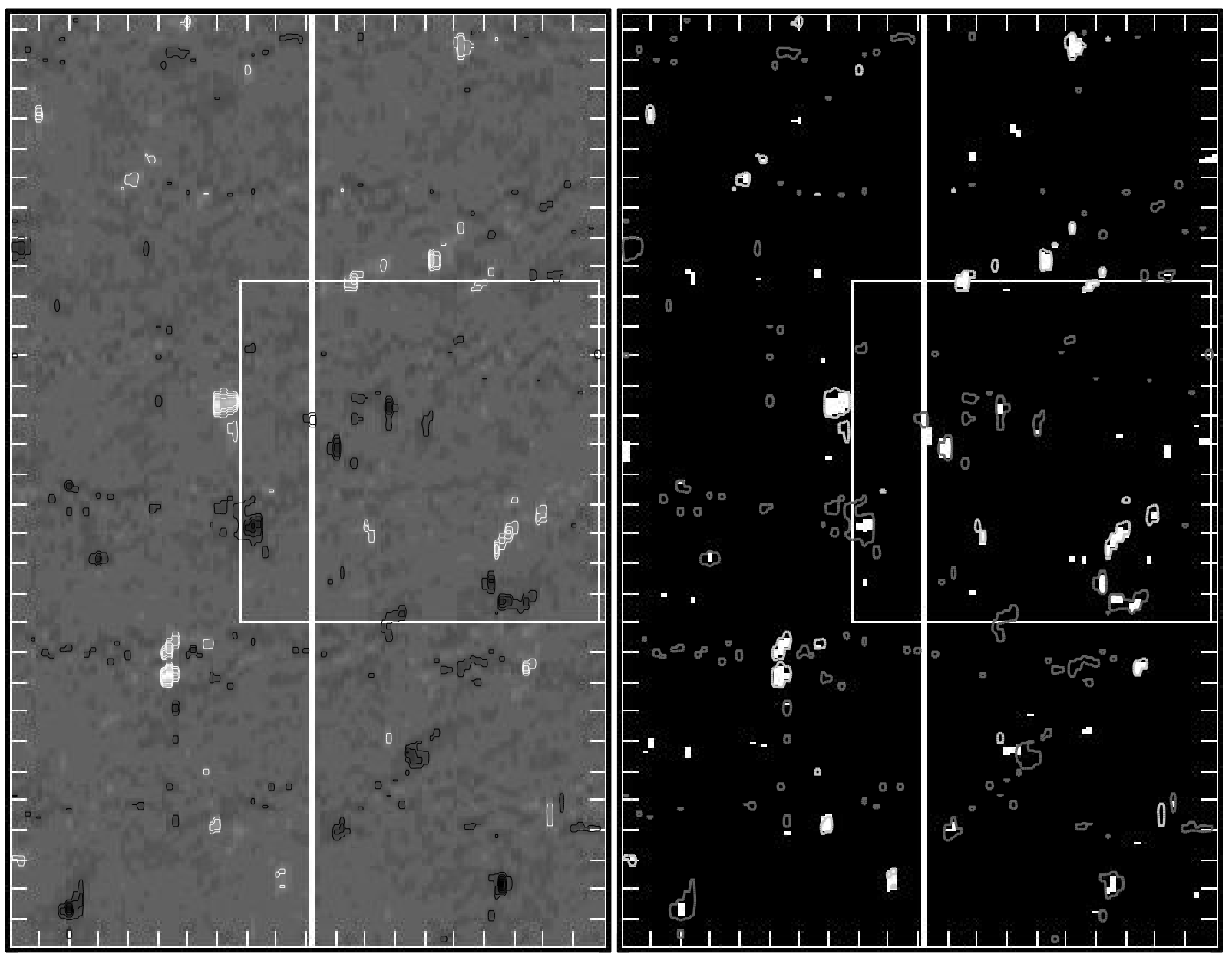

Abbildung 7.3: links: GCT-Magnetogramm mit eingezeichneter SUMER-Spaltposition (vertikale weiße Linie) und GCT-Bildserien-Startposition (weißes Rechteck). Zur Verdeutlichung der magnetischen Struktur sind Isokonturlinien bei $\pm 3,0 \cdot 10^{8} \mathrm{~Wb}, \pm 5,0 \cdot 10^{8} \mathrm{~Wb}, \pm 7,5 \cdot 10^{8} \mathrm{~Wb}$ und $\pm 1,0 \cdot 10^{9} \mathrm{~Wb}$ eingezeichnet. rechts: Stark vereinfachte Version des Linienzentrumsbildes. Der Hintergrund wurde, der besseren Übersicht halber, gleichmäßig schwarz und alle Aufhellungen ab $10 \%$ des Maximalkontrastes weiß gezeichnet. Zusätzlich wurden die Isokonturlinien des Magnetogramms bei $\pm 3,0 \cdot 10^{8} \mathrm{~Wb}$ übernommen. Man erkennt, daß fast alle Aufhellungen des Intensitätsbildes mit magnetischer Aktivität einhergehen. Der Abstand zwischen den Markierungen beträgt in beiden Bildern fünf Bogensekunden. 

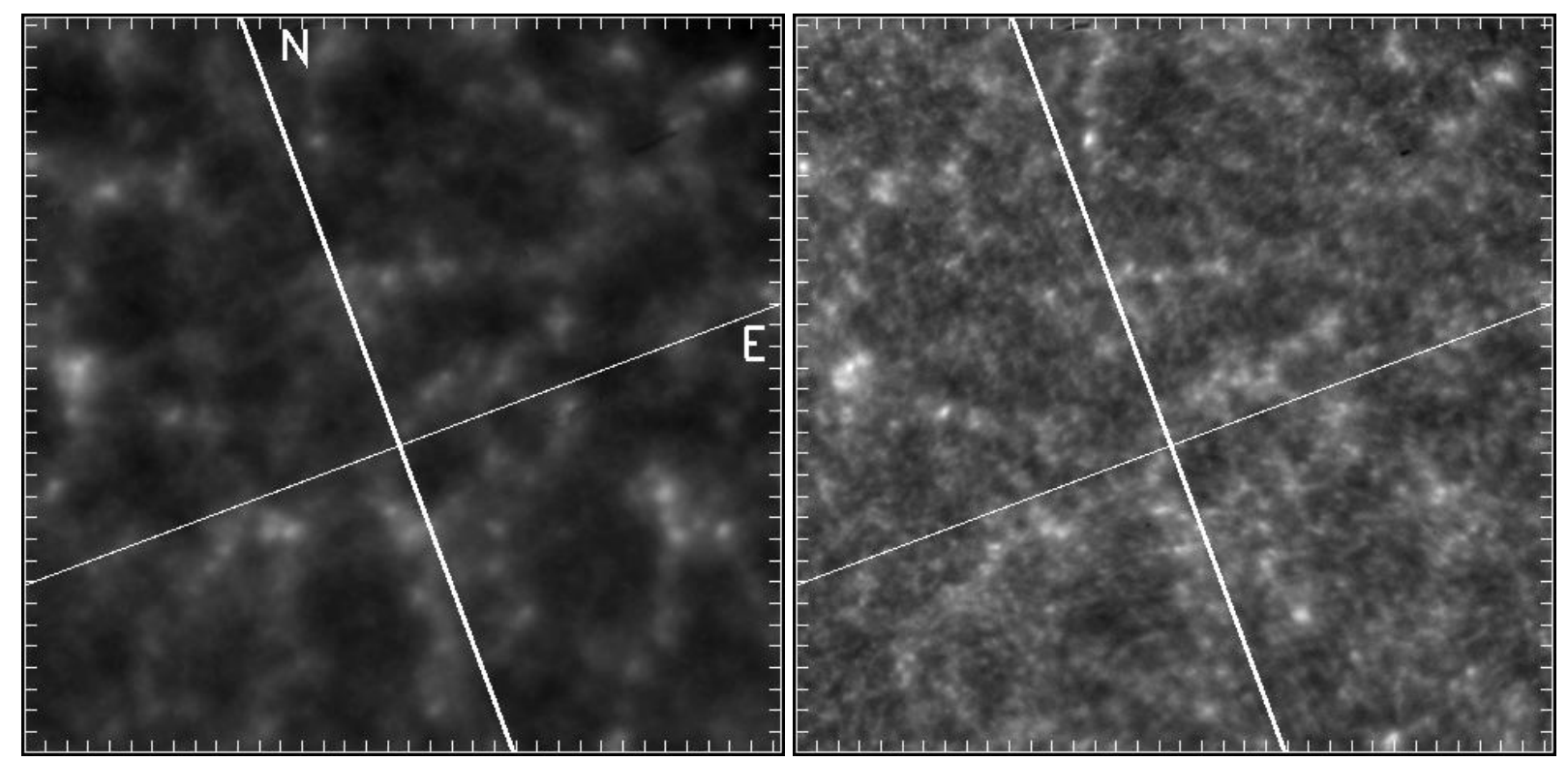

Abbildung 7.4: links: Zeitlich gemitteltes Ca II $K$-Filtergramm. Deutlich ist die großräumige zellartige Struktur der Supergranulation zu erkennen. Die Zellen haben im Mittel einen Durchmesser von ca. 30000 km. Eingezeichnet ist ferner ein Richtungskreuz zur Orientierung (schräg oben Norden $(\mathrm{N})$, rechts Osten (E)). Für die weitere Auswertung mußten die Bilder des VTTs zunächst um $20,{ }^{\circ} 4$ im Uhrzeigersinn gedreht und an der NordSüd-Achse dann gespiegelt werden. Dann ist, wie bei allen Bildern der anderen Teleskope auch, Norden oben, Süden unten, Osten links und Westen rechts. rechts: Einzelbild mit Richtungskreuz. Auch hier kann man die Zellen der Supergranulation in der für Ca-Bilder typischen "wolkigen" Struktur erkennen. Der Abstand zwischen den Markierungen beträgt in beiden Bildern fünf Bogensekunden. 

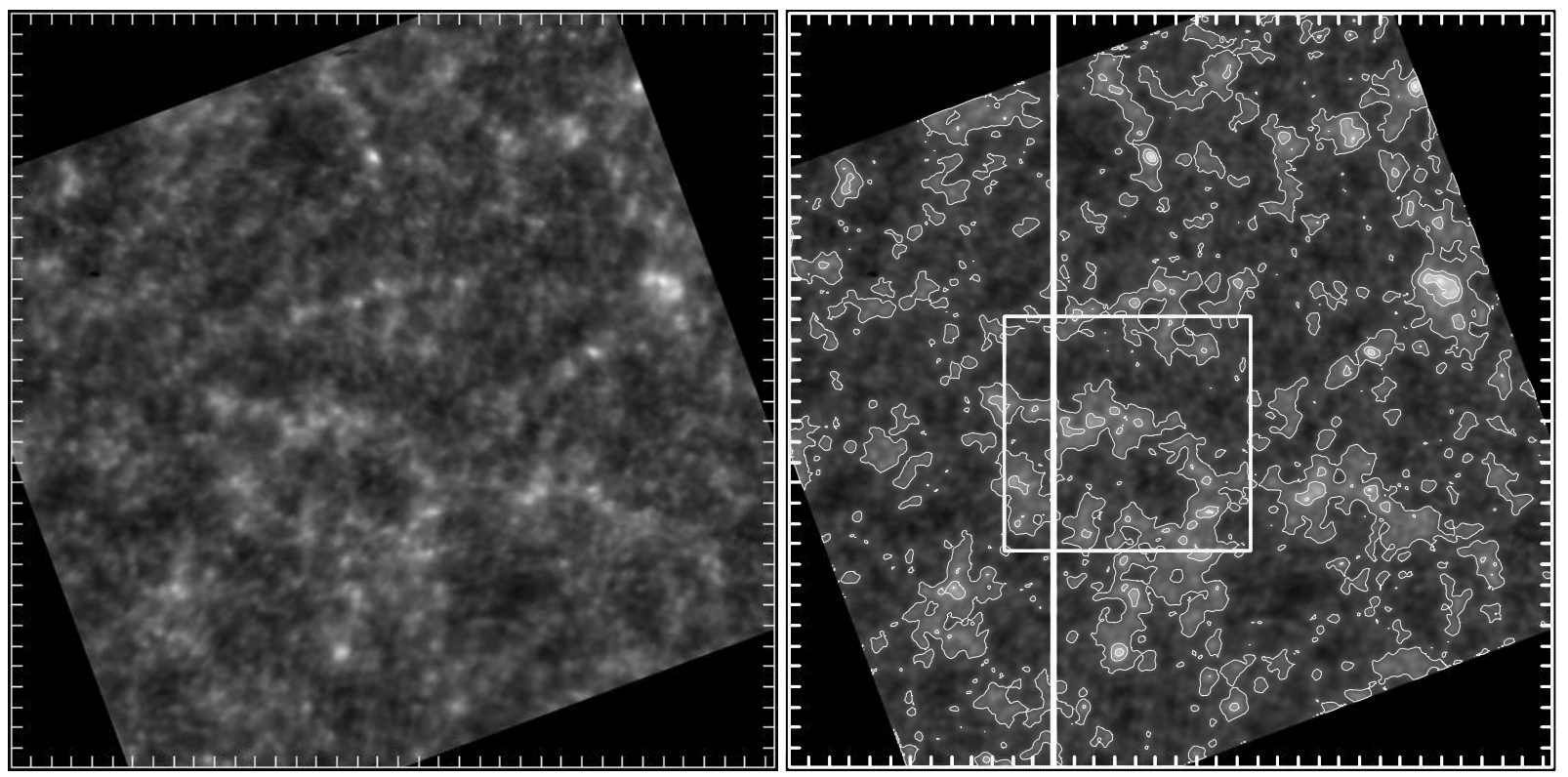

Abbildung 7.5: links: Am 10.5.1999 um 8:18 UT aufgenommenes Ca II-Filtergramm als Kontextbild. Das ursprünglich 510 Pixel $\times 504$ Pixel große Bild $\left(\approx 187^{\prime \prime} \times\right.$ $184^{\prime \prime}$ bei $\left.0,366 \frac{\prime \prime}{\text { Pixel }}\right)$ wurde durch abschneiden eines gewissen Bereiches an den Rändern auf eine Größe von 482 Pixeln $\times 468$ Pixeln gebracht. Die durch die Derotation entstandenen leeren Flächen finden sich in den Ecken. Man erkennt über das gesamte Bild verteilt kleine, lokale Aufhellungen an oder auf den Rändern der supergranularen Zellen. rechts: Das gleiche Bild wie links, aber mit eingezeichneter SUMERSpaltposition (weiße Linie) und GCT-Bildserien-Startposition (Rechteck). Weiterhin heben Isokonturlinien die supergranulare Struktur deutlich hervor. Die Isokonturlinien befinden sich bei 30\%, 50\% und $70 \%$ des Maximalkontrastes. Die annähernd elliptische supergranulare Zelle im unteren Bereich des kleinen Rechtecks hat eine große Achse von

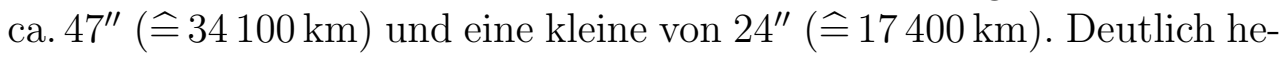
ben sich eine ganze Reihe von kleinen Aufhellungen, die alle in der Nähe oder auf dem Rand der Supergranulenzelle liegen, vom Untergrund ab. Der Abstand zwischen den Markierungen beträgt in beiden Bildern fünf Bogensekunden. 
Minuten liegt und sieht deshalb von kleinen Verschiebungen ab, so läßt sich feststellen, daß erhöhte magnetische Aktivität mit erhöhter Intensität im Ca-Filtergramm verbunden ist.

In Abb. 7.7 ist schließlich zweimal dasselbe He I-Filtergramm des SUMER-Teleskops zu sehen. Das linke Bild zeigt die von 7:56:34 UT bis 8:15:14 UT aufgenommene $79^{\prime \prime} \times 356^{\prime \prime}$ große "unveränderte" Version. Im rechten Bild ist die SUMER-Spaltposition zu Beginn der rotationskompensierten Spektren der Zeitserie sowie die Startposition der GCTBildserie eingezeichnet. Weiterhin wurde in Abb. 7.7 rechts der Kontrast erheblich verstärkt, um die schwächeren Strukturen gerade im Bereich des Rechtecks stärker hervortreten zu lassen. Das in den Filtergrammen weiter unten liegende Gebiet hoher Intensität erscheint daher ein wenig überbelichtet. 


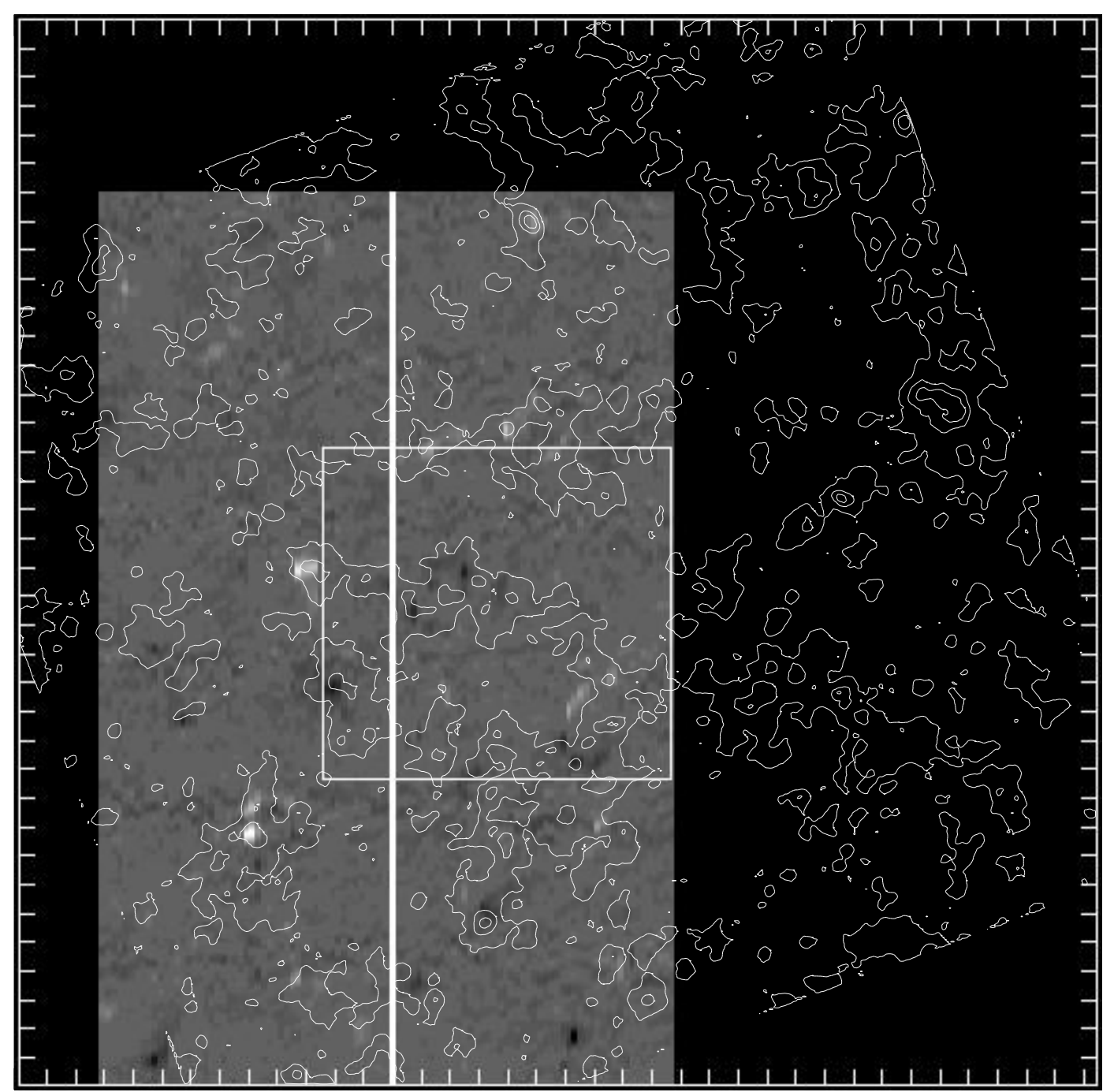

Abbildung 7.6: Kompositbild aus GCT-Magnetogramm und Isokonturlinienabbildung des VTT-Ca-Filtergramms. Ferner sind die SUMER-Spaltposition und GCT-Bildserien-Startposition eingezeichnet. Man erkennt, daß auf den Rändern der Supergranule die Gebiete magnetischer Aktivität liegen. Bedenkt man, daß zwischen dem GCT-Kontextbild und dem CaFiltergramm des VTTs eine zeitliche Differenz von ca. 15 Minuten liegt und sieht deshalb von kleinen Verschiebungen ab, so läßt sich feststellen, daß erhöhte magnetische Aktivität mit erhöhter Intensität im CaFiltergramm verbunden ist. Der Abstand zwischen den Markierungen beträgt fünf Bogensekunden. 

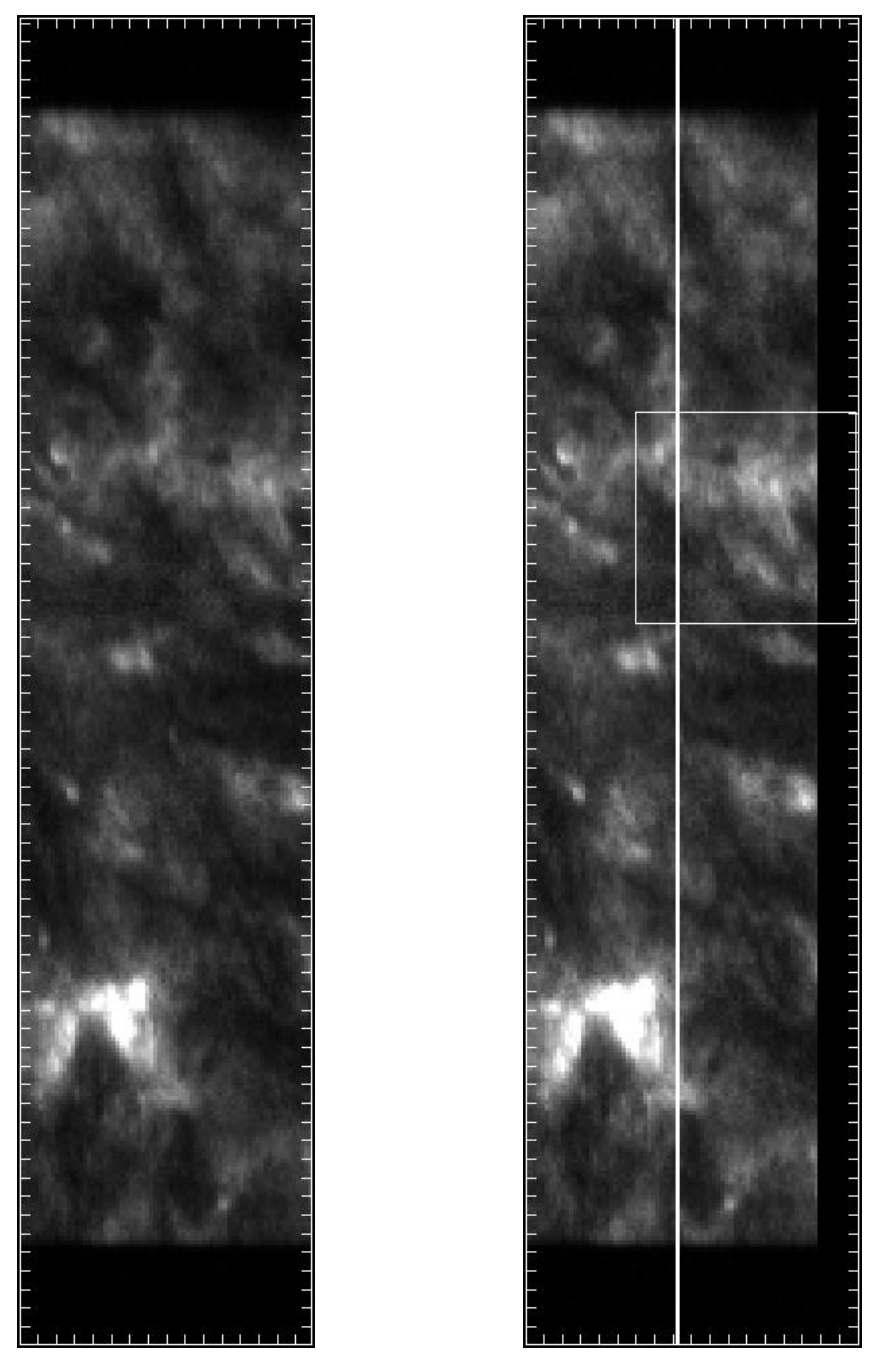

Abbildung 7.7: links: Die von 7:56:34 UT bis 8:15:14 UT aufgenommene $79^{\prime \prime} \times 356^{\prime \prime}$ große "unveränderte" Version des He I-Filtergramms. rechts: Die kontrastverstärkte Version mit eingezeichneter SUMER-Spaltposition für die rotationskompensierten Spektren der Zeitserie und der Startposition der GCT-Bildserie. Der Abstand zwischen den Markierungen beträgt in beiden Bildern fünf Bogensekunden. 


\section{Die GCT-Bildserie}

Die gesamte Serie von 120 GCT-Magnetogrammen des 10.5.1999 ist in den Abbildungen 8.1 bis $8.4 \mathrm{zu}$ sehen. Die Bilder sind von links nach rechts und von oben nach unten in zeitlicher Abfolge aneinander gereiht. Die einzelnen Bilder haben jeweils eine räumliche Ausdehnung von $60^{\prime \prime} \times 57^{\prime \prime}$ (entspricht $43500 \mathrm{~km} \times 41325 \mathrm{~km}$ auf der Sonne; die Markierungen sind in Abständen von 5" gesetzt). Die hellen, weißen Gebiete tragen positive Polarität (magnetisch Norden), die schwarzen Bereiche negative Polarität (magnetisch Süden) und der graue Hintergrund markiert das Nullniveau. Alle Polaritäten $< \pm 2,6 \cdot 10^{8} \mathrm{~Wb}$ wurden auf Null gesetzt. Die Aufnahme des ersten Spektrums des ersten Scans des ersten Bildes (Abb. 8.1 oben links) wurde um 8:09:13 UT begonnen. Der zweite Scan des ersten Bildes (jedes Bild besteht aus 2 Scans von je 60 Spektren) begann um 8:10:30 UT und endete um 8:11:41 UT. Es vergingen also $77 \mathrm{~s}$ bis der erste und $71 \mathrm{~s}$ bis der zweite Scan aufgenommen war. Die Aufnahme des ganzen ersten Bildes überstreicht also ein Zeitintervall von $148 \mathrm{~s}$ (2 min $28 \mathrm{~s}$ ). Die unterschiedlichen Längen der Zeitintervalle der beiden Scans eines Bildes und damit auch der Bilder untereinander sind auf die halbautomatische Auslegung des Aufbaus zurückzuführen. Der gesamte Beobachtungszeitraum von 8:09:13 UT bis 12:34:56 UT überstreicht einen Zeitraum von $15943 \mathrm{~s}$ (4 h 25 min $43 \mathrm{~s}$ ). Daraus folgt, daß im Mittel alle $\approx 133 \mathrm{~s}\left(\frac{15943 \mathrm{~s}}{120 \text { Bilder }}\right)$ ein Bild entstand.

Folgt man mit den Augen den Bildern in zeitlicher Reihenfolge (von links nach rechts und von oben nach unten), läßt also die Bilder in der Art eines Films an sich vorbeiziehen, bemerkt man, daß sich die magnetischen Strukturen rasch ändern und Gebiete mal intensiver, mal weniger intensiv erscheinen. Der dabei maximal auftretende magnetische Fluß positiver Polarität beträgt $+7,28 \cdot 10^{9} \mathrm{~Wb}$ (bei einem Aufösungselement von $\left(0,{ }^{\prime \prime} 77\right)^{2}$ entspricht das einer mittleren Flußdichte von $\left.\approx 234 \mathrm{G}=23,4 \mathrm{mT}\right)$. Für die negative Polarität findet sich ein Minimum von $-4,16 \cdot 10^{9} \mathrm{~Wb}$ (entsprechend einer mittleren Flußdichte von $\approx 134 \mathrm{G}=13,4 \mathrm{mT}$ ). Den beobachteten individuellen Variationen ist eine großräumige Bewegung aller Strukturen der Magnetogramme unterlegt (Abb. 8.5 unten rechts). Eine Analyse dieser Bewegung hat gezeigt, daß es sich um eine technische Bewegung handelt, die ihren Ursprung im optischen Aufbau hat. Um die Bewegung der Bilder untereinander zu kompensieren, muß man sie geeignet gegeneinander verschieben. Das verkleinert natürlich die Größe des gemeinsamen in allen Bildern enthaltenen Beobachtungsbereichs. Man verschiebt zunächst ein jedes Bild gegen das vorhergehende und addiert anschließend die Werte aller vorhergehenden Einzelverschiebungen bis zu 


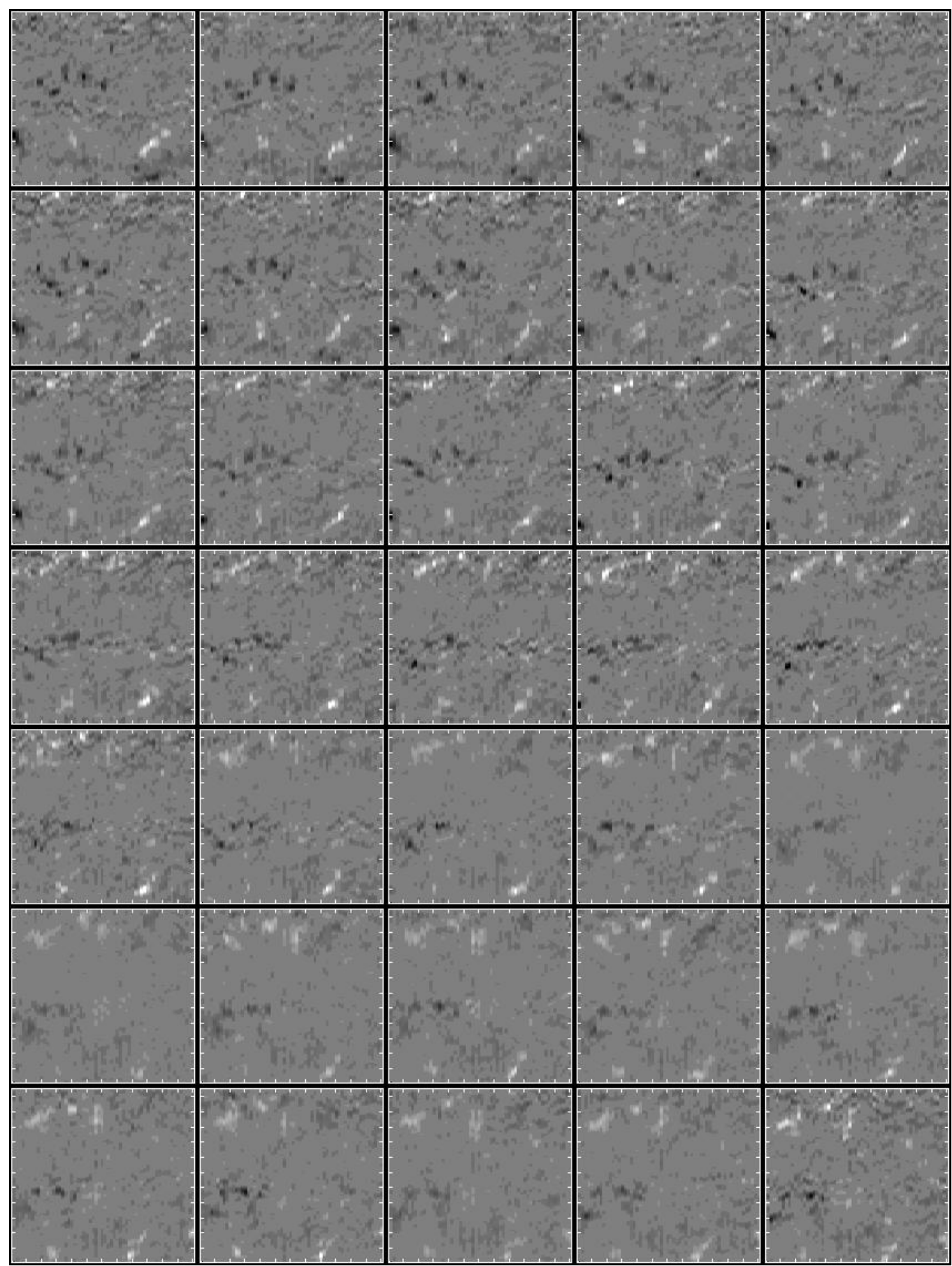

Abbildung 8.1: Von links nach rechts und oben nach unten sind die GCTMagnetogramme Nr. 0 bis 34 des 10.5.1999 in zeitlicher Abfolge dargestellt. Der Abstand zwischen den Markierungen beträgt 5". 


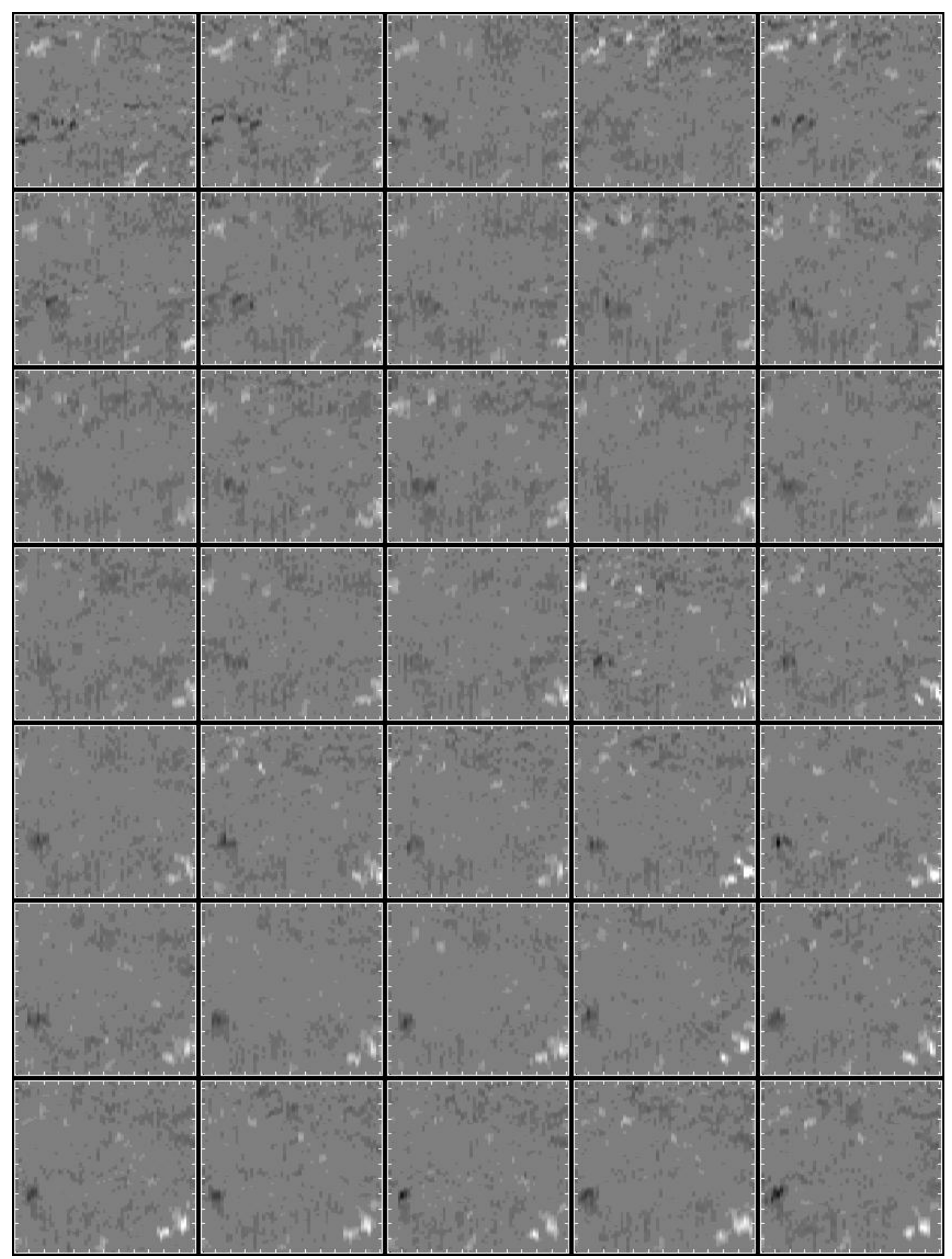

Abbildung 8.2: Fortsetzung Abb. 8.1, Magnetogramme Nr. 35 bis 69. 


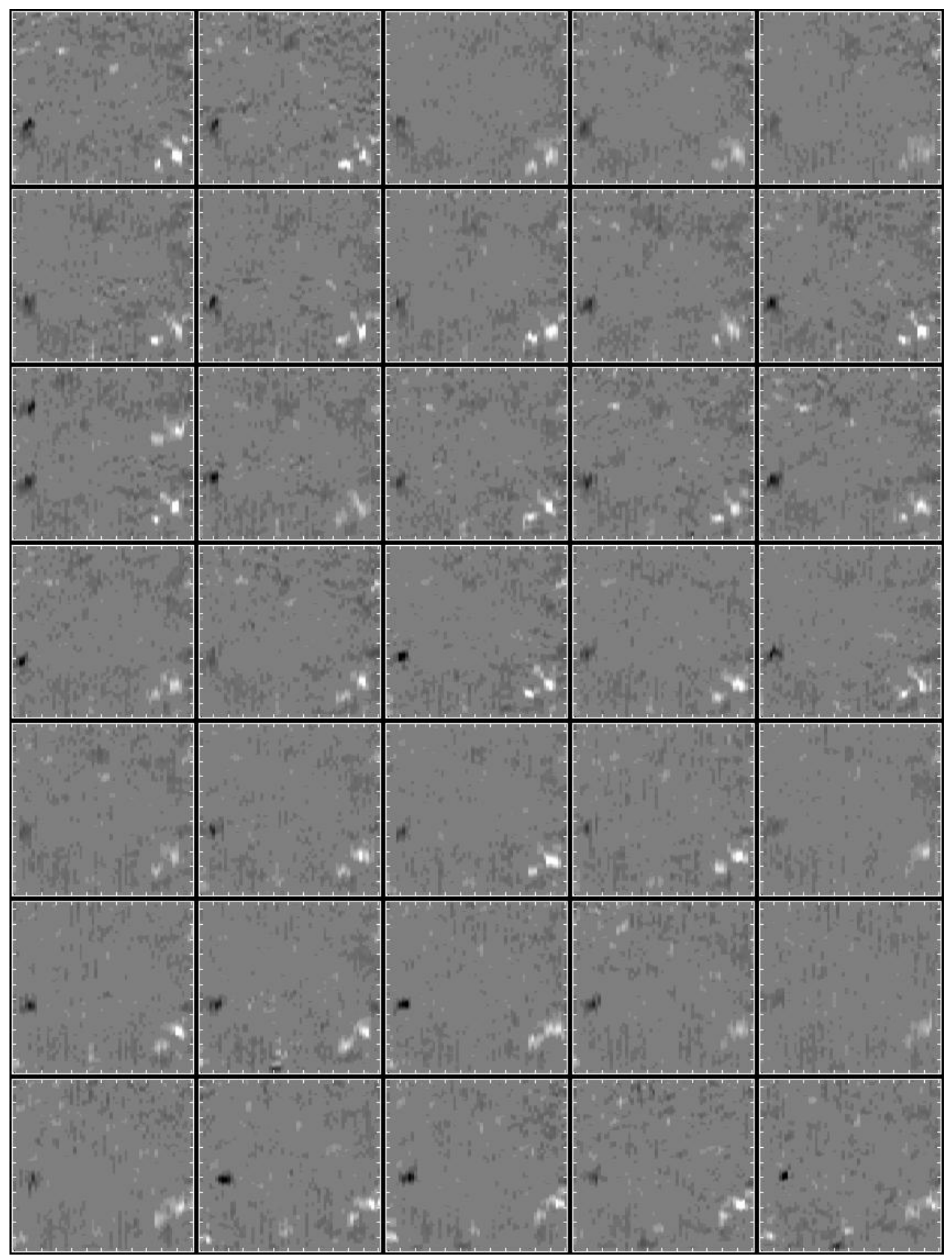

Abbildung 8.3: Fortsetzung Abb. 8.1, Magnetogramme Nr. 70 bis 104. 


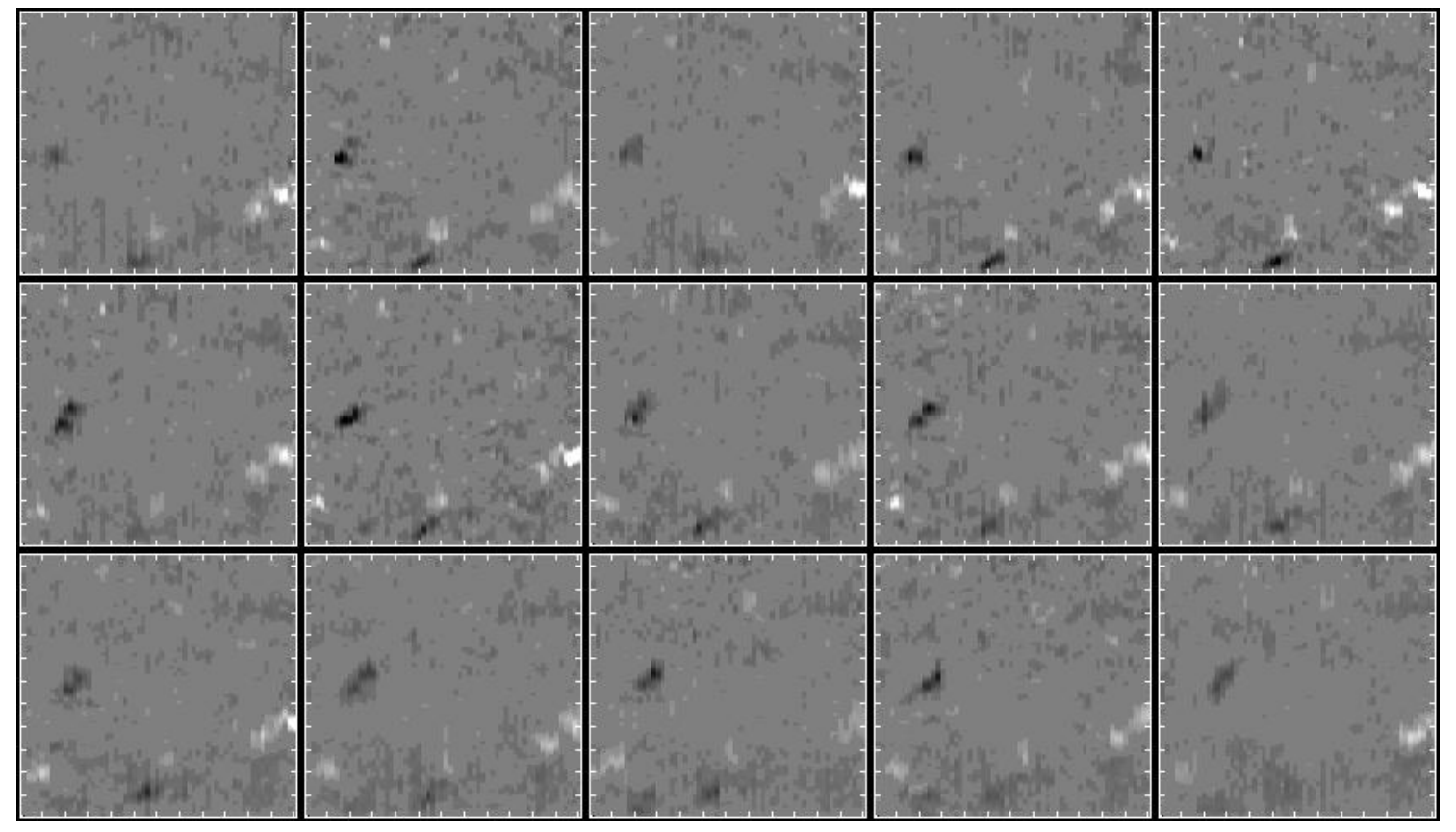

Abbildung 8.4: Fortsetzung Abb. 8.1, Magnetogramme Nr. 105 bis 119. Die hellen, weißen Gebiete tragen positive Polarität (magnetisch Norden), die schwarzen Bereiche negative Polarität (magnetisch Süden) und der graue Hintergrund markiert das Nullniveau. Alle Polaritäten $< \pm 2,6 \cdot 10^{8} \mathrm{~Wb}$ wurden auf Null gesetzt. Der maximal auftretende magnetische Fluß positiver Polarität beträgt $+7,28 \cdot 10^{9} \mathrm{~Wb}$ (bei einem Auflösungselement von $\left(0,{ }^{\prime \prime} 77\right)^{2}$ entspricht das einer mittleren Flußdichte von $\approx$ $234 \mathrm{G}=23,4 \mathrm{mT}$ ). Für die negative Polarität findet sich ein Minimum von $-4,16 \cdot 10^{9} \mathrm{~Wb}$ (entsprechend einer mittleren Flußdichte von $\approx$ $134 \mathrm{G}=13,4 \mathrm{mT})$. 
dem jeweilig aktuellen Bild. So bezieht man die Verschiebungen auf das erste Bild der Serie. In Abb. 8.5 links und rechts oben sind die Verschiebungen in $x$ - bzw. $y$-Richtung gegen die Bildnummer aufgetragen. Die durchgezogene Linie stellt die beste Näherungskurve durch die Punkte dar. Deutlich erkennt man einen cosinus- bzw. sinusförmigen Verlauf der Kurve. Das bedeutet, daß eine ellipsenförmige Bewegung resultiert. In Abb. 8.5 unten links ist die Verschiebung in $y$-Richtung als Funktion der Verschiebung in $x$ Richtung dargestellt. Durch Punktspiegelung dieser, zur Kompensation der Bewegung aller Strukturen eines Bildes benötigten Kurve findet sich die beobachtete Bewegung (vgl. Abb. 8.5 unten rechts; der Richtungssinn ist durch eine Pfeilspitze markiert).

Alle Magnetogramme wurden gemäß der entsprechenden Verschiebungswerte in einen Rahmen eingesetzt und mit der SUMER-Spaltposition versehen (siehe Abb. 8.6 bis 8.9). Dem ohnehin schon mißlichen Umstand, die SUMER-Spaltposition nur etwa 10" vom östlichen Rand des Bildes entfernt vorzufinden, addiert sich jetzt nachteilig die Bildbewegung. Der Beobachtungsbereich der GCT-Magnetogramme verliert ab Bildnummer 46 um 9:54 UT die SUMER-Spaltposition. Gemildert wird dieser Verlust allerdings ein wenig dadurch, daß an diesem Tag mit SUMER ohnehin "nur" 10146 s beobachtet wurde. Das $x$-Diagramm der Abb. 8.10 entstand durch die Aneinanderfügung aller Bildinformationen der GCT-Magnetogramme an der SUMER-Spaltposition in zeitlicher Abfolge. Die kompensierte Bewegung äußert sich hier in dem krummen Verlauf der Bildränder. 

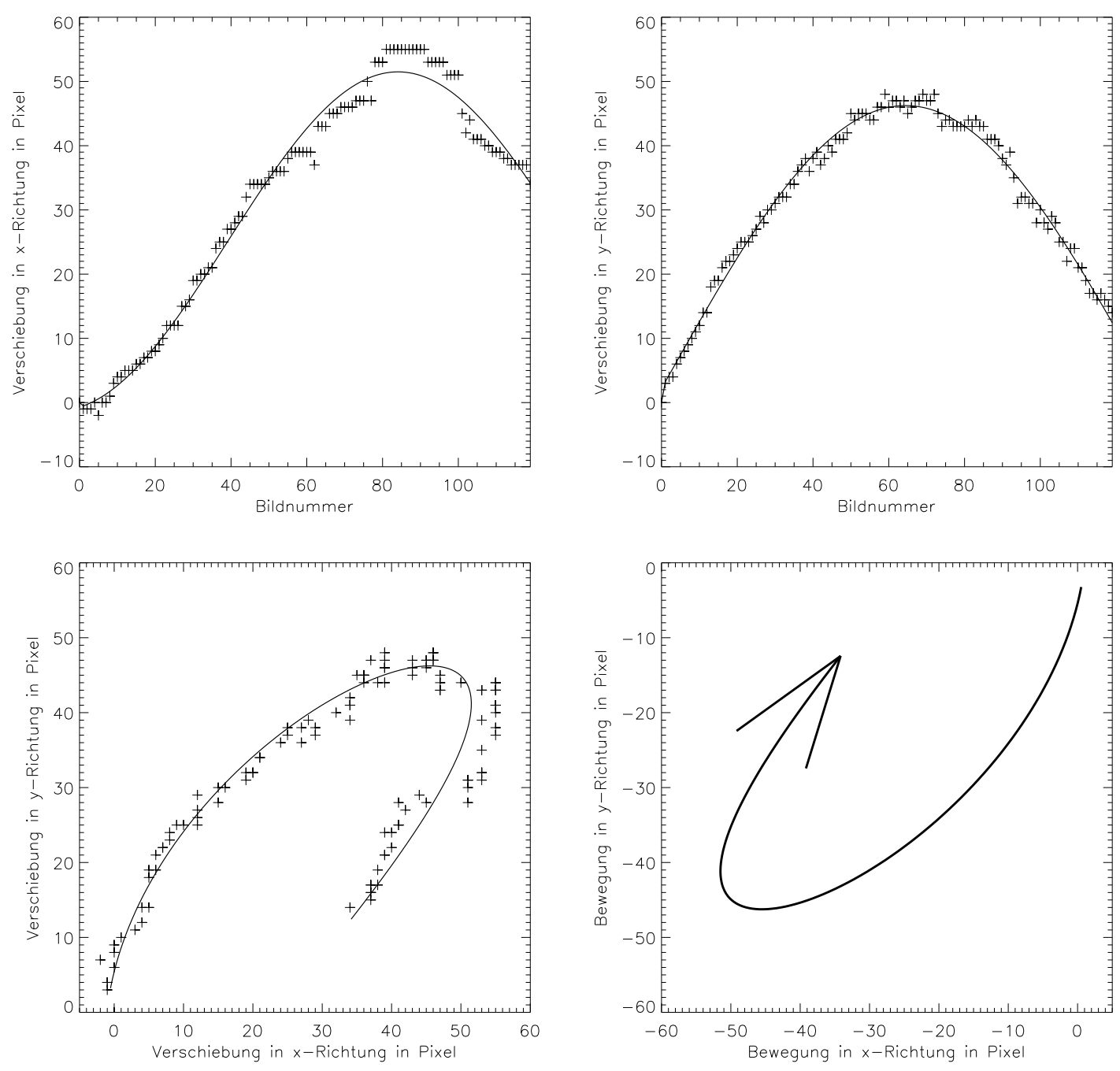

Abbildung 8.5: Auftragungen der Werte für die Kompensation der technischen Bildbewegung. Oben links und rechts: Auftragung der auf das erste Bild bezogenen $x$ - bzw. $y$-Verschiebungswerte gegen die Bildnummer. Die durchgezogene Linie ist die beste Näherungskurve durch die Punkte. Unten links: Aus den $x$ - und $y$-Werten zusammengesetzte Kurve zur Bewegungskompensation mit der besten Näherungskurve (durchgezogene Linie). Unten rechts: Beobachtete und dann kompensierte gemeinsame Bewegung aller Strukturen auf den Bildern. Die Pfeilspitze gibt die Bewegungsrichtung in zeitlicher Ordnung an (1 Pixel entspricht $0,{ }^{\prime \prime} 366$ ). 


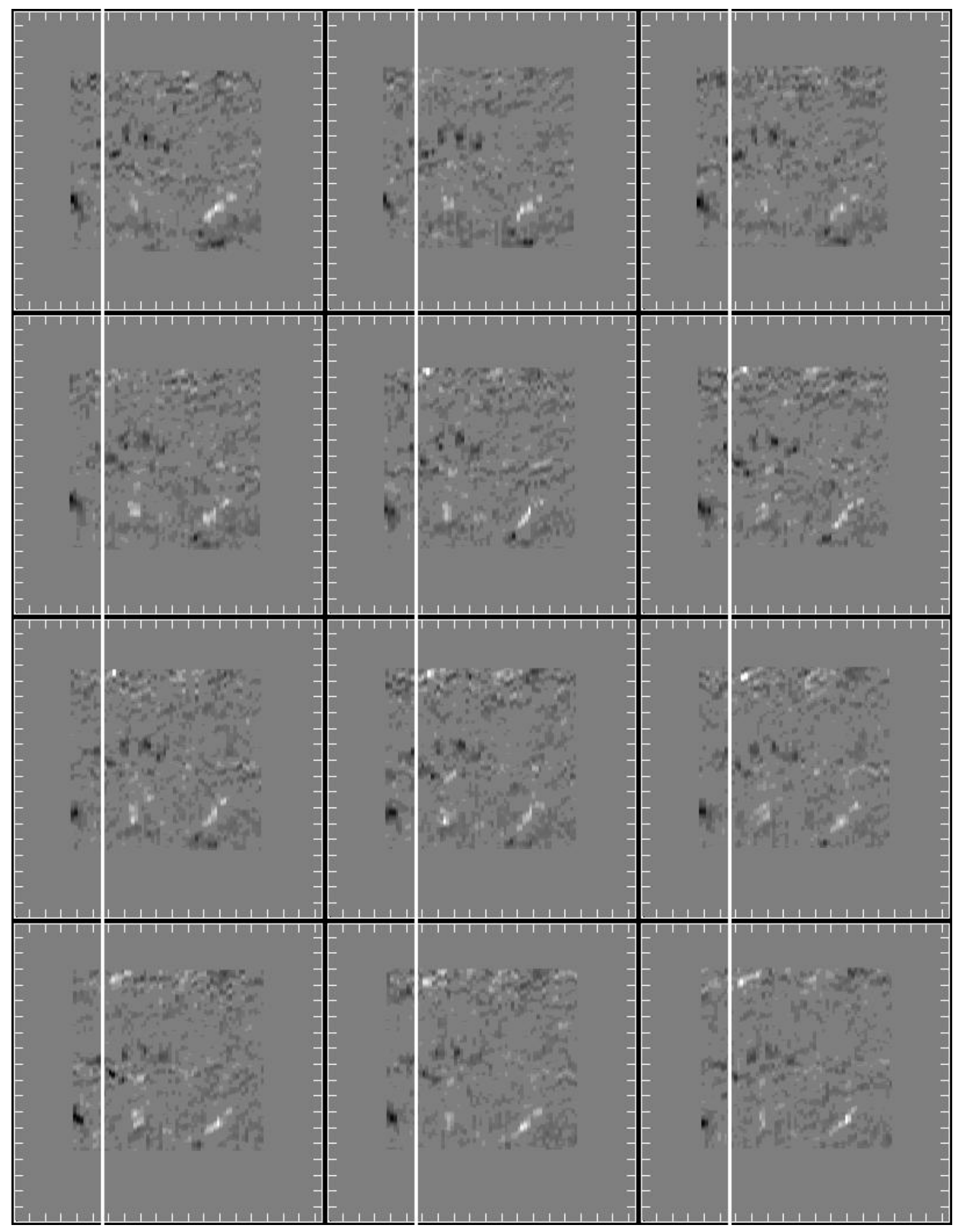

Abbildung 8.6: Von links nach rechts und oben nach unten in einen Rahmen unter Berücksichtigung der Bewegungswerte eingesetzte Magnetogramme Nr. 0 bis 11. Die weiße Linie markiert die SUMER-Spaltposition. Der Abstand zwischen den Markierungen beträgt $5^{\prime \prime}$. 


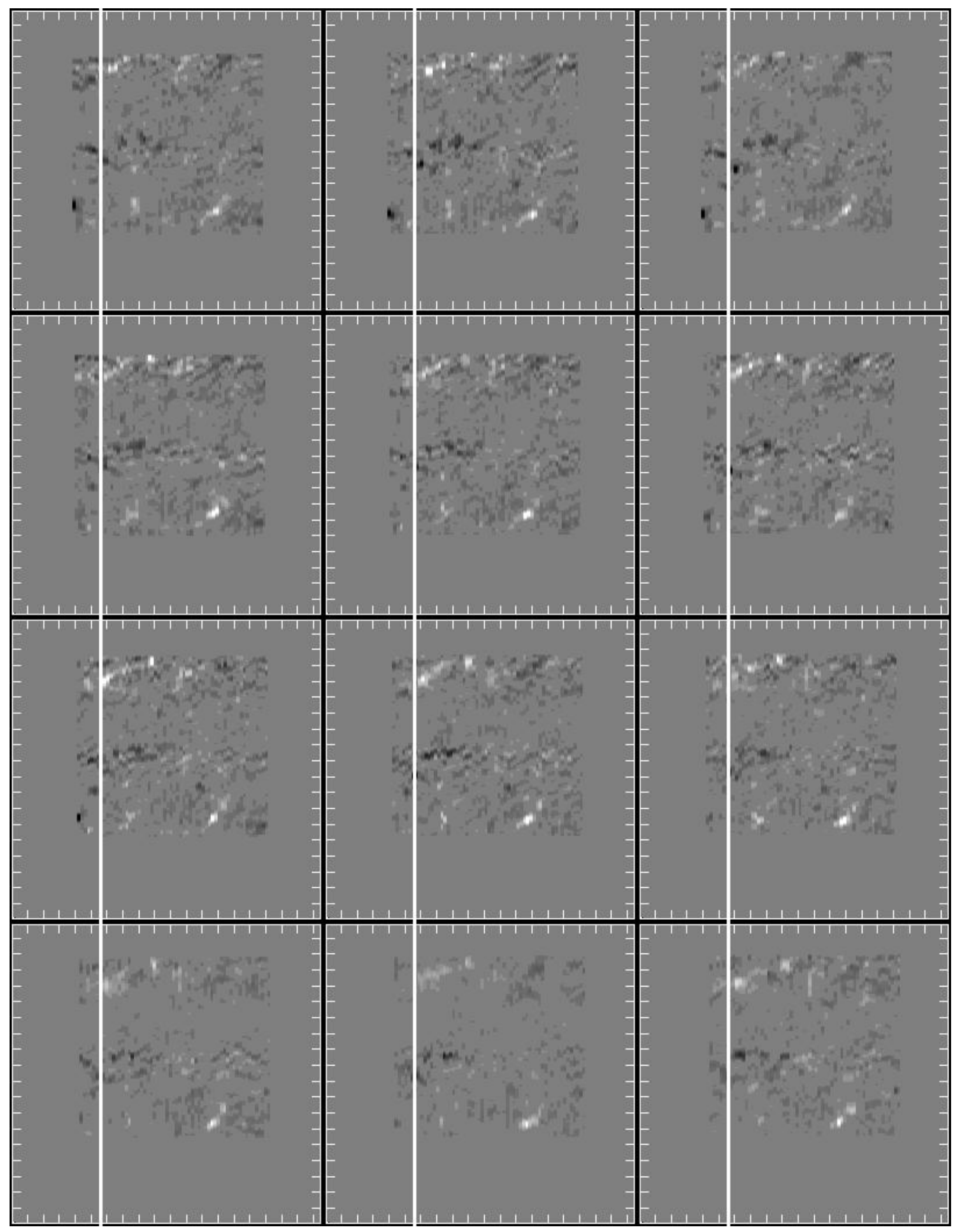

Abbildung 8.7: Fortsetzung Abb. 8.6, Magnetogramme Nr. 12 bis 23. 


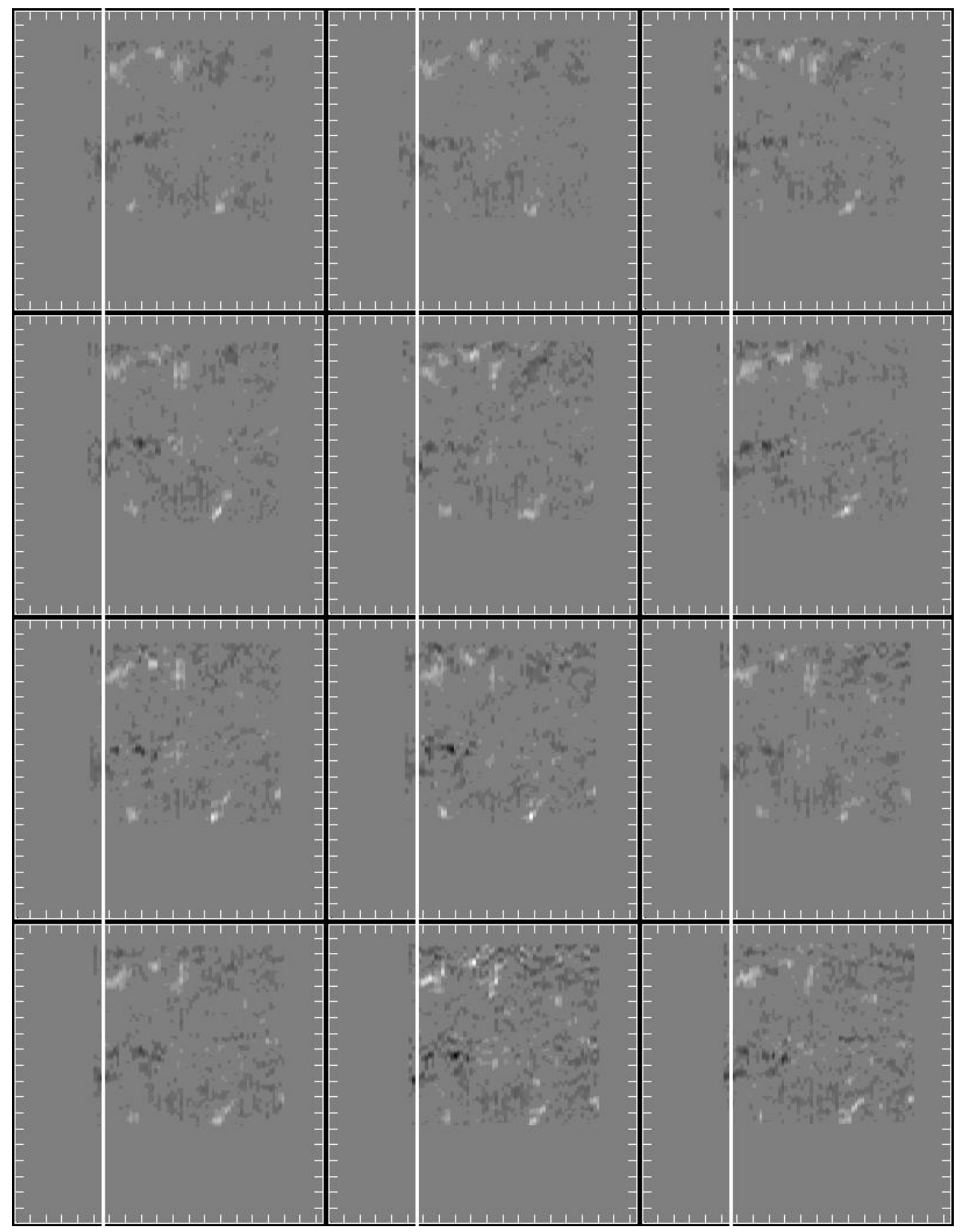

Abbildung 8.8: Fortsetzung Abb. 8.6, Magnetogramme Nr. 24 bis 35. 


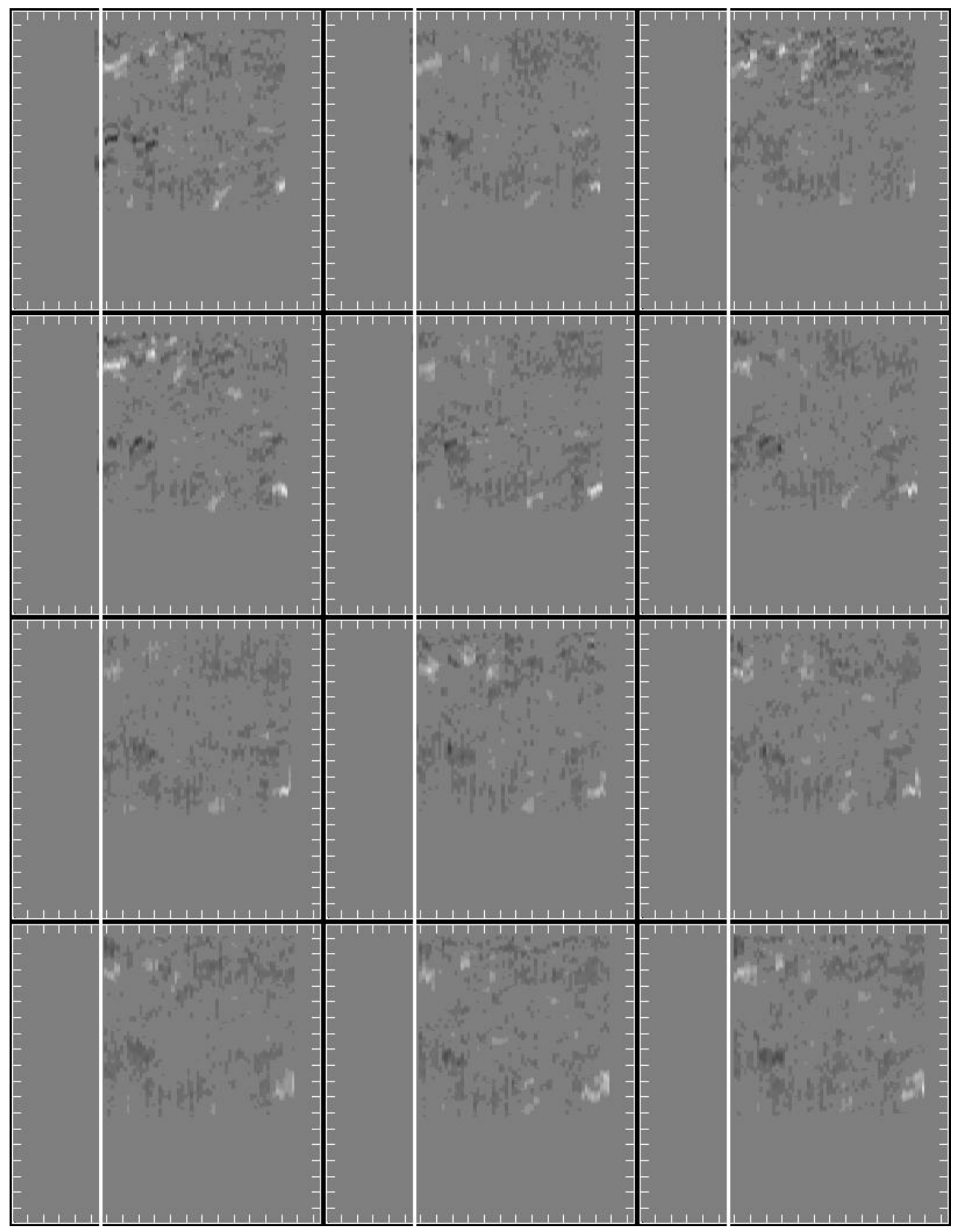

Abbildung 8.9: Fortsetzung Abb. 8.6, Magnetogramme Nr. 36 bis 47. 


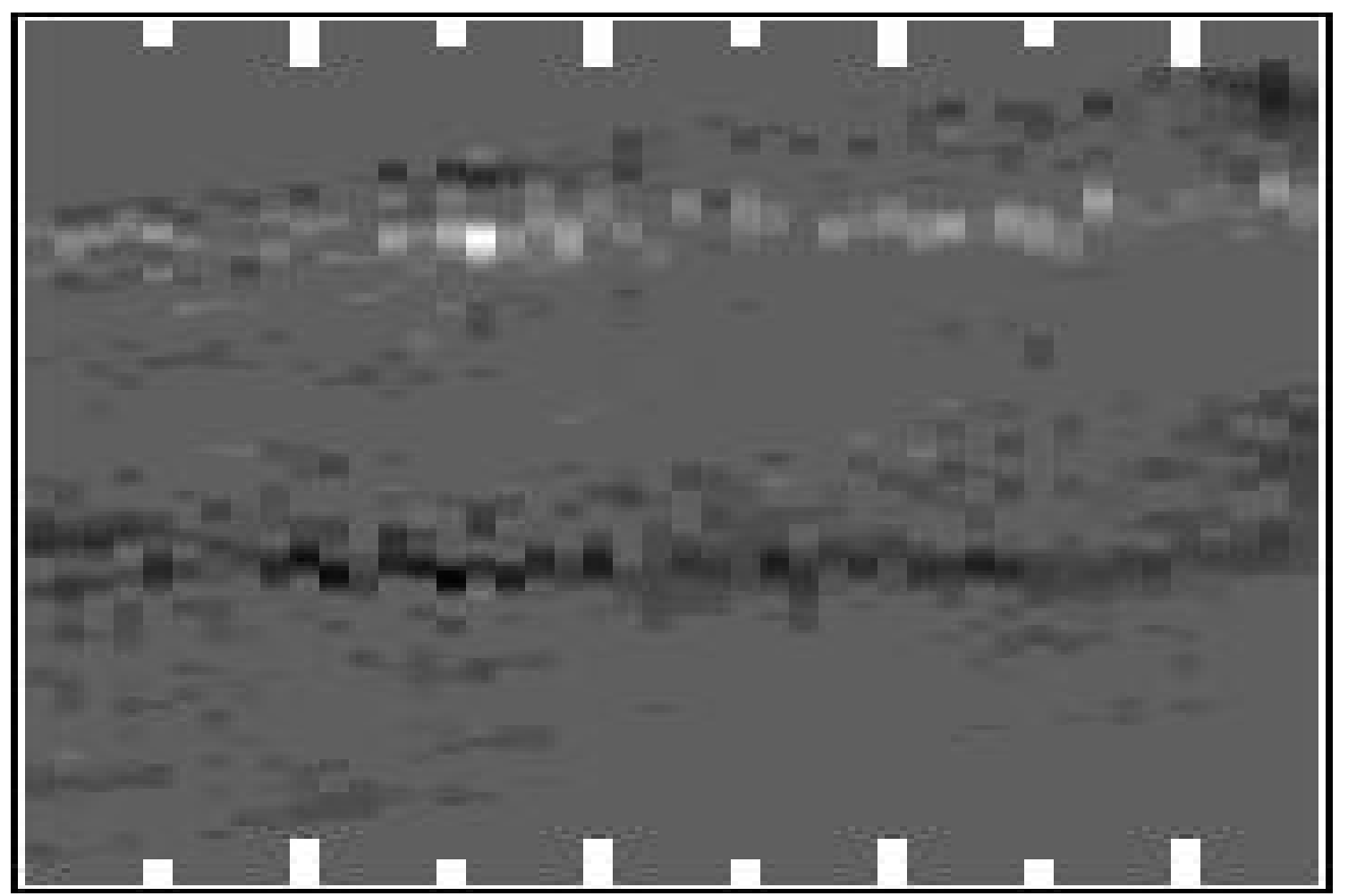

Abbildung 8.10: $x$-Diagramm aus GCT-Magnetogrammen an der Stelle des SUMERSpaltes. Dieses $x t$-Diagramm entstand durch die Aneinanderfügung aller Bildinformationen der GCT-Magnetogramme (Nr. 3 bis 46) an der SUMER-Spaltposition in zeitlicher Abfolge. Diese 44 Magnetogramme wurden gewählt, da sie den mit SUMER gemeinsamen Beobachtungszeitraum von $5844 \mathrm{~s}$ (1 h $37 \mathrm{~min} 24 \mathrm{~s}$ ) abdecken. Die Markierungsstriche in Zeit-Richtung wurden an jeder fünften Position gesetzt; überflüssige Bereiche des Rahmens wurden entfernt. 


\section{Ergebnisse der Modellrechnungen}

In diesem Kapitel werden die Ergebnisse der Potentialfeldextrapolationen gezeigt. Wie schon in Kap. 3.3 erwähnt, sind die über den Zeeman-Effekt auf photosphärischem Niveau ermittelten Magnetogramme Abb. 8.1 bis 8.4 bzw. 8.6 bis 8.9 Ausgangspunkt der Extrapolationen in höhere Schichten der Sonnenatmosphäre.

Die Abb. 9.1 zeigt das erste Magnetogramm (Nr. 0; vgl. Abb. 8.1 links oben bzw. 8.6 links oben) der GCT-Bildserie zunächst nur in Isokonturliniendarstellung. Allen folgenden Potentialfeldliniendarstellungen ist das jeweilige Ausgangsmagnetogramm in dieser Form unterlegt. Die Isokonturlinien sind dabei immer bei mittleren magnetischen Flußdichten von $\pm 10 \mathrm{G}, \pm 30 \mathrm{G}, \pm 50 \mathrm{G}, \pm 70 \mathrm{G}, \pm 90 \mathrm{G}, \pm 110 \mathrm{G}, \pm 130 \mathrm{G}, \pm 150 \mathrm{G}, \pm 170 \mathrm{G}, \pm 190 \mathrm{G}$ und $\pm 210 \mathrm{G}$ eingezeichnet. Dies entspricht bei dem verwendeten Auflösungselement einem Bereich von etwa $\pm 3 \cdot 10^{8} \mathrm{~Wb}$ bis ca. $\pm 6,6 \cdot 10^{9} \mathrm{~Wb}$. Die Magnetogramme haben jeweils eine Ausdehnung von $60^{\prime \prime} \times 57^{\prime \prime}$. Die Auflösung wurde aber aus numerischen Gründen auf $0,75 \frac{1 "}{\text { Pixel }}$ reduziert, wobei der gesamte auf die betrachtete Fläche entfallende magnetische Fluß erhalten wurde. Die durchgezogenen Isokonturlinien wurden für die hellen Bereiche positiver Polarität (magnetisch Norden) und die gestrichelten für die dunklen Bereiche negativer Polarität (magnetisch Süden) verwendet. In Abb. 9.2 sind die extrapolierten Potentialfeldlinien auf dem Hintergrund der Abb. 9.1 dargestellt. Es wurden hier zunächst nur geschlossene Feldlinien eingezeichnet. Abb. 9.3 zeigt Abb. 9.2 in räumlicher Darstellung. Die $z$-Achse (Höhe) ist, anders als die in Bogensekunden unterteilte $x$ - bzw. $y$-Achse, in Einheiten von $1000 \mathrm{~km}$ eingeteilt. Abb. 9.4 zeigt in Draufsicht und Abb. 9.5 in räumlicher Darstellung das vollständige Ergebnis der Potentialfeldextrapolation des ersten Magnetogramms der Zeitserie, wobei allerdings in Abb. 9.4 aus Gründen der Übersichtlichkeit jede zweite berechnete Feldlinie nicht eingezeichnet wurde. Da der magnetische Fluß negativer Polarität (gestrichelte Isokonturlinien) im betrachteten räumlichen Gebiet von $60^{\prime \prime} \times 57^{\prime \prime}$ bei weitem überwiegt, sind in diesen Bereichen nur lokal offene Feldlinien anzutreffen. Sie gehören zu magnetischen Gebieten außerhalb des vorliegenden. Offene Feldlinien werden in den die Zeitserie (Nr. 0 bis 47, vgl. Abb 8.6 bis 8.9) wiedergebenden Darstellungen Abb. 9.8 bis 9.15 wegen der besseren Übersichtlichkeit fortgelassen. In den beiden folgenden Abb. 9.6 und 9.7 soll anhand der Potentialfeldextrapolation des ersten Magnetogramms (vgl. 9.1 bis 9.5) die Höhenstruktur offengelegt werden. Hierzu wurden in die entsprechende Darstellung jeweils nur Potentialfeldlinien eingezeichnet, die bis in ein gewisses Höhenintervall $\Delta z=1000 \mathrm{~km}$ reichen (bis auf Darstellung Abb. 9.7 unten rechts). Die Abb. 9.6 oben links weist eine Vielzahl von 


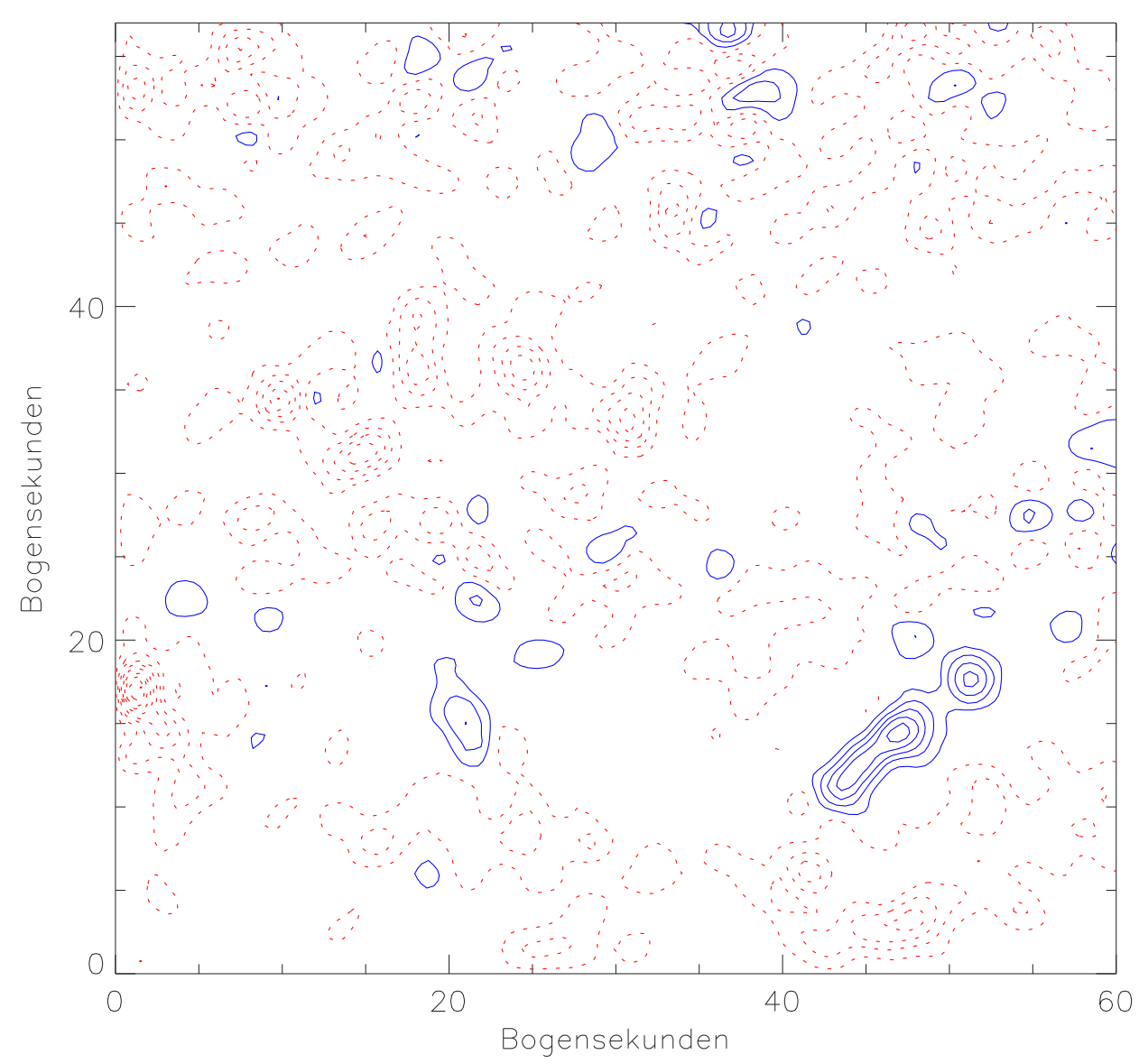

Abbildung 9.1: Erstes Magnetogramm (Nr. 0; $\quad$ vgl. Abb. 8.1 bzw. 8.6) der GCT-Bildserie in Isokonturliniendarstellung. Die Isokonturlinien sind bei mittleren magnetischen Flußdichten von $\pm 10 \mathrm{G}, \pm 30 \mathrm{G}, \pm 50 \mathrm{G}, \pm 70 \mathrm{G}, \pm 90 \mathrm{G}, \pm 110 \mathrm{G}, \pm 130 \mathrm{G}, \pm 150 \mathrm{G}, \pm 170 \mathrm{G}$, $\pm 190 \mathrm{G}$ und $\pm 210 \mathrm{G}$ eingezeichnet. Dies entspricht bei dem verwendeten Auflösungselement einem Bereich von etwa $\pm 3 \cdot 10^{8} \mathrm{~Wb}$ bis ca. $\pm 6,6 \cdot 10^{9} \mathrm{~Wb}$. Das Magnetogramm hat eine Ausdehnung von $60^{\prime \prime} \times 57^{\prime \prime}$. Die Auflösung wurde aus numerischen Gründen auf $0,75 \frac{1}{\text { Pixel }}$ reduziert, wobei der gesamte auf die betrachtete Fläche entfallende magnetische Fluß erhalten wurde. Die durchgezogenen Isokonturlinien wurden für die hellen Bereiche positiver Polarität (magnetisch Norden) und die gestrichelten für die dunklen Bereiche negativer Polarität (magnetisch Süden) verwendet. 


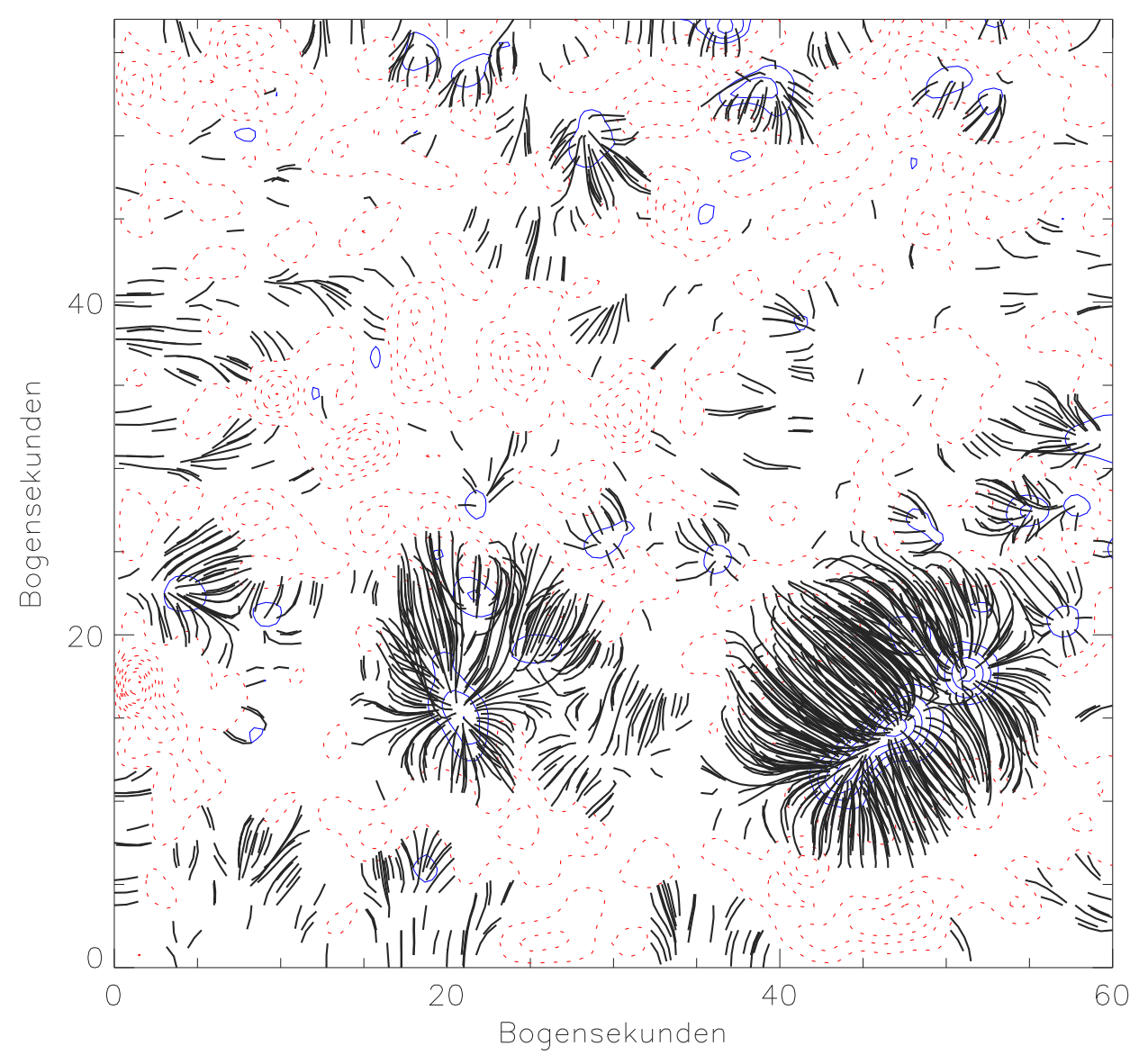

Abbildung 9.2: Selbes Bild wie Abb. 9.1 mit zusätzlich eingezeichneten, extrapolierten Potentialfeldlinien. Der Übersichtlichkeit halber wurden nur zur Oberfläche zurückkehrende Feldlinien eingezeichnet. 


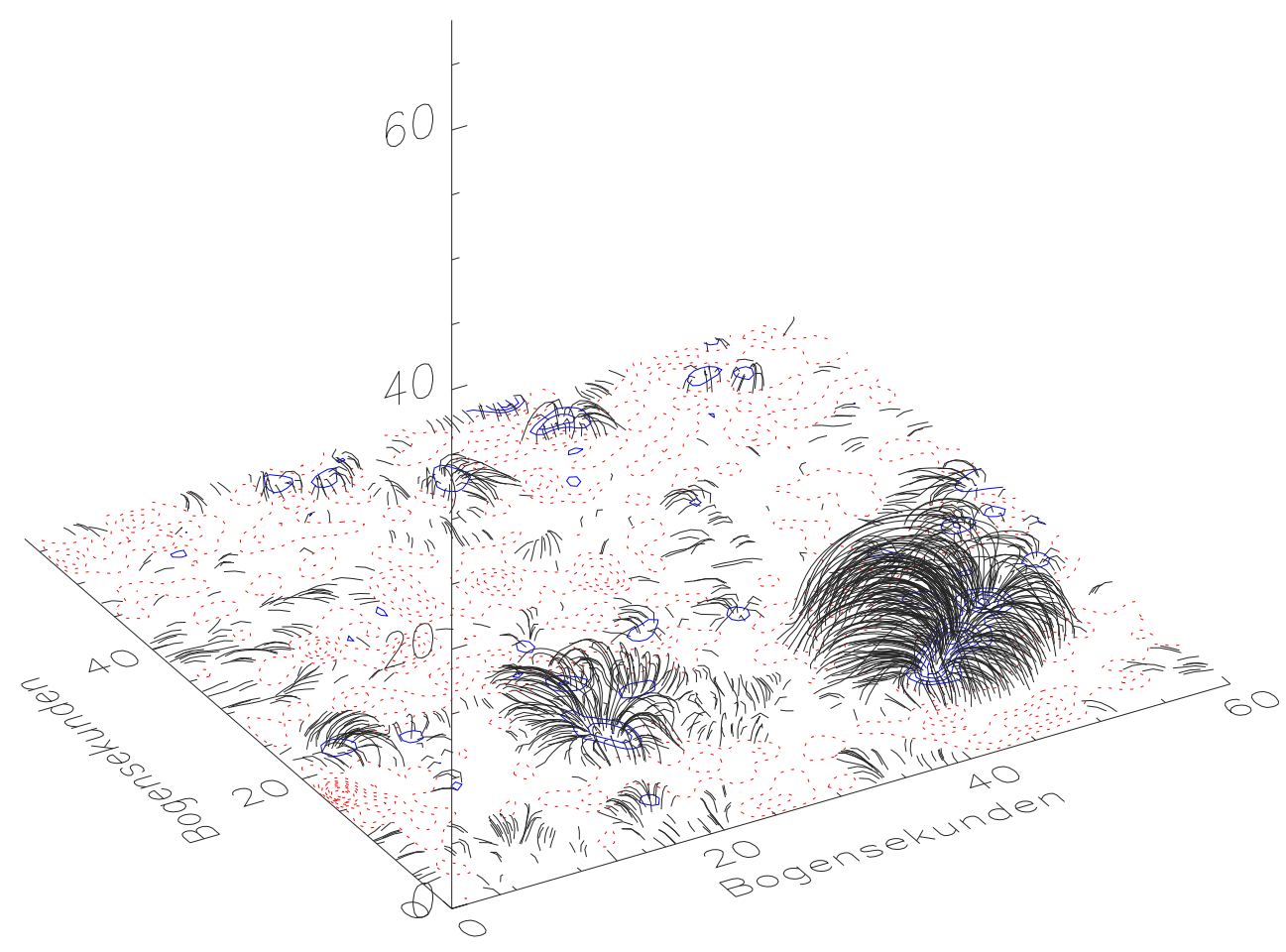

Abbildung 9.3: Abb. 9.2 in räumlicher Darstellung. Die z-Achse (Höhe) ist, anders als die in Bogensekunden unterteilte $x$ - bzw. $y$-Achse, in Einheiten von $1000 \mathrm{~km}$ eingeteilt. 


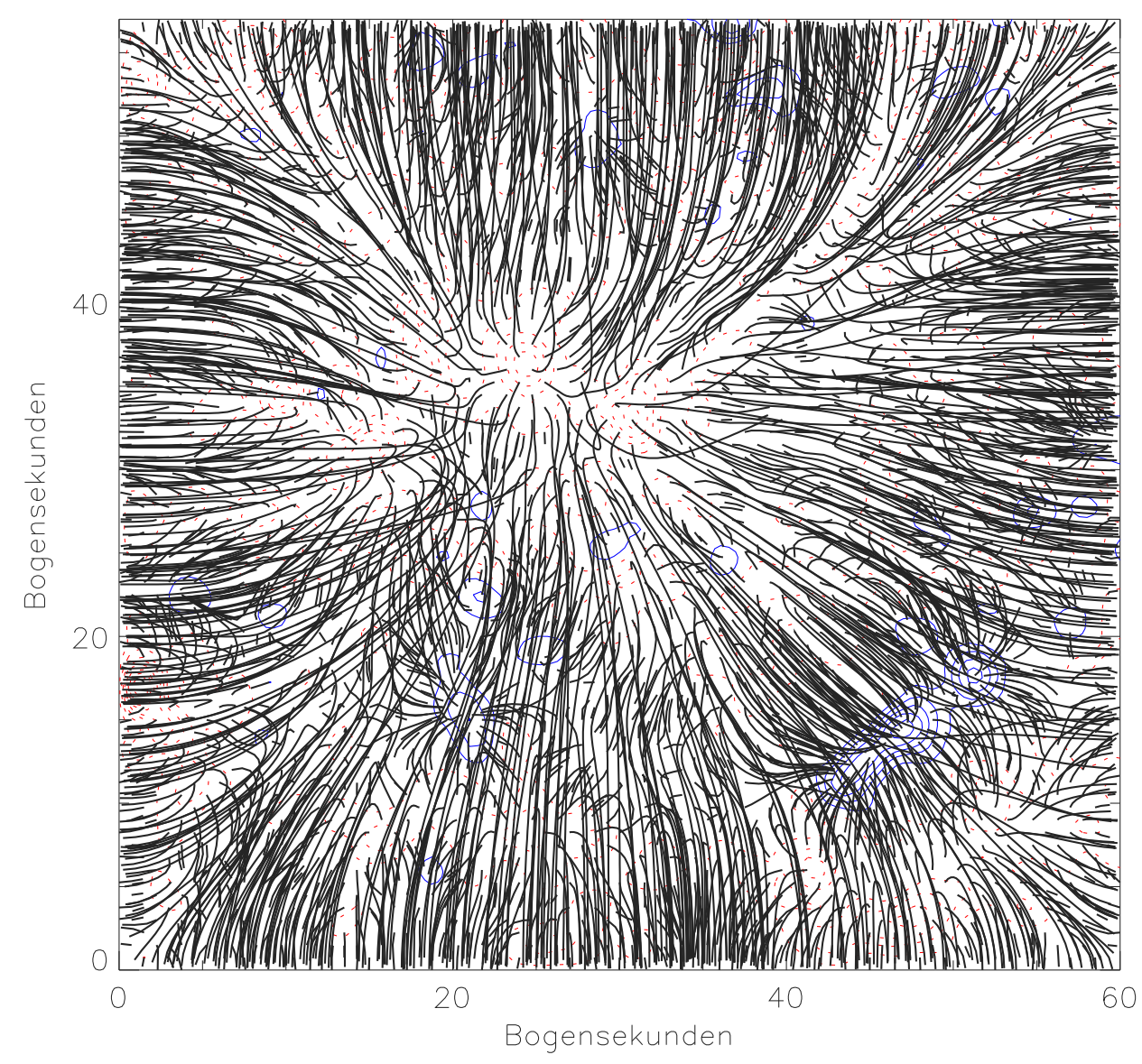

Abbildung 9.4: Abb. 9.2 mit allen berechneten Feldlinien (nur jede zweite Feldlinie ist eingezeichnet). 


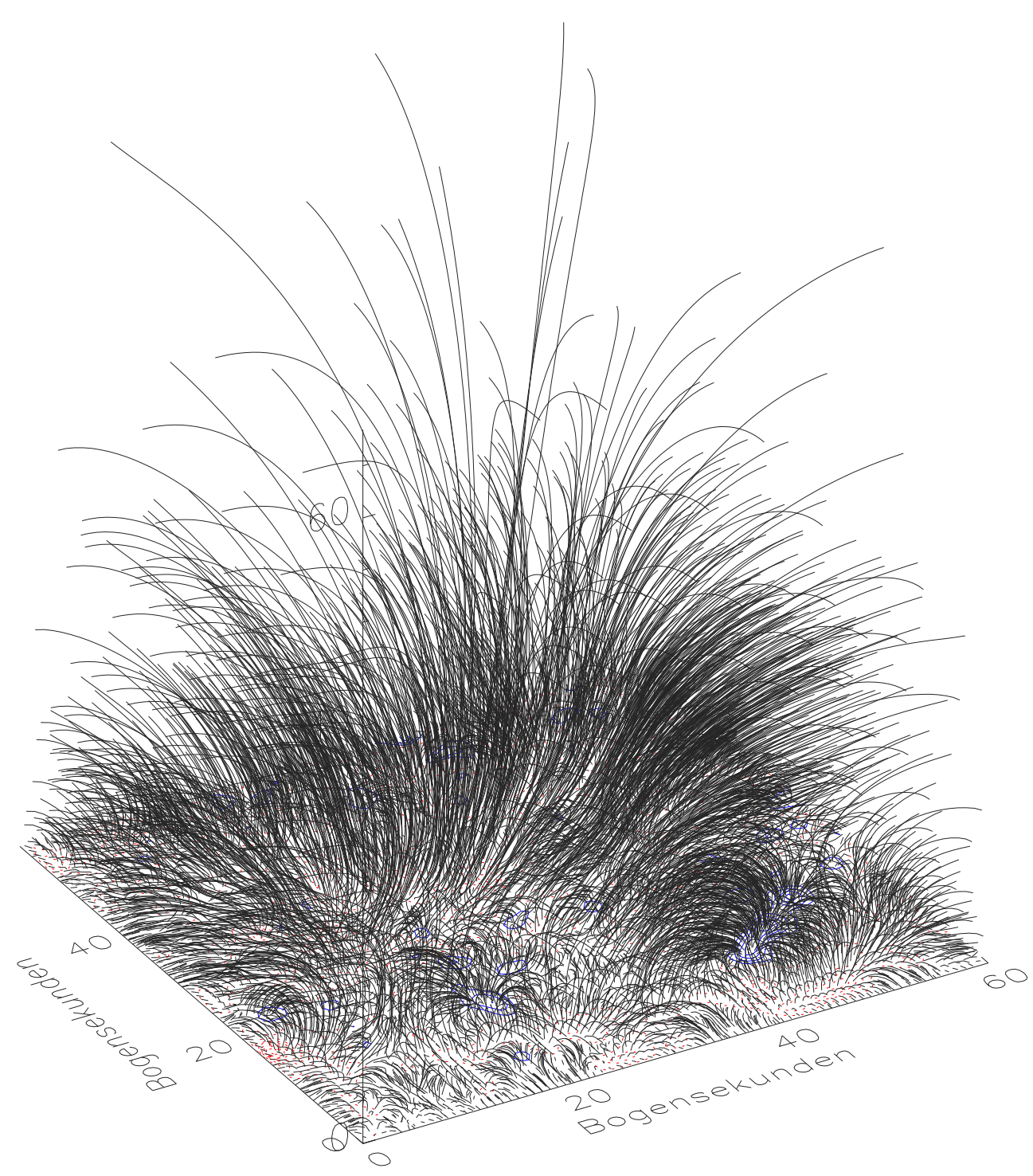

Abbildung 9.5: Abb. 9.4 in räumlicher Darstellung (alle berechneten Feldlinien sind eingezeichnet). Die $z$-Achse (Höhe) ist in Einheiten von $1000 \mathrm{~km}$ unterteilt. 
kleinen Potentialfeldbögen auf, die Höhen von weniger als $1000 \mathrm{~km}$ haben und deshalb in dem zweiten Bild (Abb. 9.6 oben rechts) verschwunden sind. Hier verbleiben, neben den lokal offenen Feldlinien am Bildfeldrand fast ausschließlich mit den Gebieten starken magnetischen Flusses positiver Polarität in der unteren Bildhälfte in Verbindung stehende, zur Sonnenoberfläche zurückkehrende, "geschlossene" Potentialfeldlinien. Im Besonderen verschwindet die letzte geschlossene Feldlinie, die mit dem Gebiet starken magnetischen Flusses positiver Polarität (unten rechts im Bild) in Verbindung steht, erst in einer Höhe zwischen $8000 \mathrm{~km}$ bis $9000 \mathrm{~km}$ (vgl. Abb. 9.7 Mitte links und rechts). Die offenen Feldlinien wandern, der Logik des Extrapolationsprogramms folgend, für immer größer werdende Höhen immer weiter zur Bildfeldmitte, bis schließlich in Abb. 9.7 unten rechts der ganze verbleibende Rest an Feldlinien aus dem großen Intervall von $11000 \mathrm{~km}$ bis $68000 \mathrm{~km}$ auf einmal eingezeichnet wird. Wie ein Schirm überdeckt dieser Teil von Feldlinien den zuvor diskutierten Teil.

In den folgenden Abbildungen sind die für die Auswertung relevanten 48 Potentialfeldextrapolationen dargestellt (eigentlich sind nur 44 wichtig, vgl. Abb. 8.10). Es sind dies die zu den Abb. 8.6 bis 8.9 gehörigen Modellrechnungen. Die in Abb. 9.8 bis 9.15 dargestellte Zeitserie überstreicht ein Zeitintervall von $\Delta t \approx 133 \frac{\mathrm{s}}{\text { Bild }} \cdot 48$ Bildern $=6384 \mathrm{~s}=$ $1 \mathrm{~h} 46$ min 24 s. Das schon erwähnte Gebiet starken magnetischen Flusses positiver Polarität unten rechts im ersten Bild (Nr. 0) bleibt in den folgenden 47 Bildern weitestgehend erhalten, die Struktur des Gebietes wandelt sich jedoch zum Teil sehr stark. Weiterhin macht das ganze Gebiet die in Kap. 8 (vgl. Abb. 8.5 unten rechts) beschriebene großräumige Bewegung mit.

Durch Vergleich mit jeweils zugehörigen Magnetogrammen der Abb. 8.6 bis 8.9 läßt sich die SUMER-Spaltposition finden. Die SUMER-Spaltposition ist zu Beginn (Bild Nr. 0) etwa 10" vom östlichen Rand entfernt und wandert dann mit der Zeit nach Osten (bzw. der Bildausschnitt nach Westen) bis er den vom GCT erfaßten Bereich verläßt. Folgt man dieser Bewegung des Spaltes auf den Potentialfeldextrapolationen, so findet man zu Beginn der Beobachtung sehr schwache magnetische Aktivität in dem Gebiet, in dem der SUMER-Spalt liegt. Diese Situatuion wandelt sich jedoch massiv durch das Auftreten mehrerer relativ großer Gebiete magnetisch starken Flusses positiver Polarität an der oberen linken Ecke des Bildes. Und auch im mittleren bis unteren, vom SUMER-Spalt noch erfaßten Gebiet finden vielfältige Veränderungen statt, die man auch schon anhand der Abb. 8.10 erahnen konnte. 

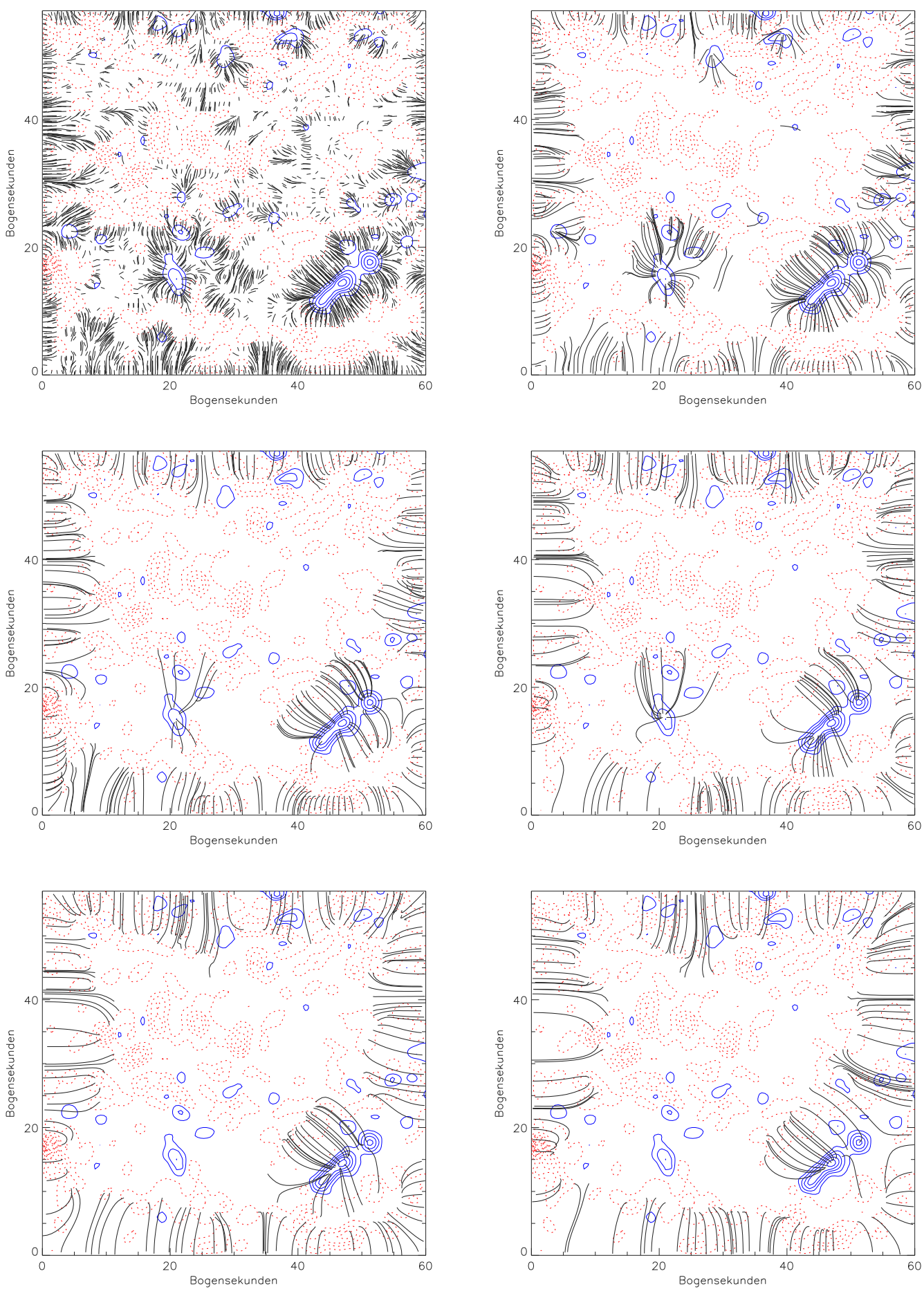

Abbildung 9.6: Von links nach rechts und oben nach unten sind jeweils nur die extrapolierten Potentialfeldlinien eingezeichnet, die in Höhen von $0 \mathrm{~km}$ bis $1000 \mathrm{~km}, 1000 \mathrm{~km}$ bis $2000 \mathrm{~km}, 2000 \mathrm{~km}$ bis $3000 \mathrm{~km}, 3000 \mathrm{~km}$ bis $4000 \mathrm{~km}, 4000 \mathrm{~km}$ bis $5000 \mathrm{~km}$ und $5000 \mathrm{~km}$ bis $6000 \mathrm{~km}$ reichen. 

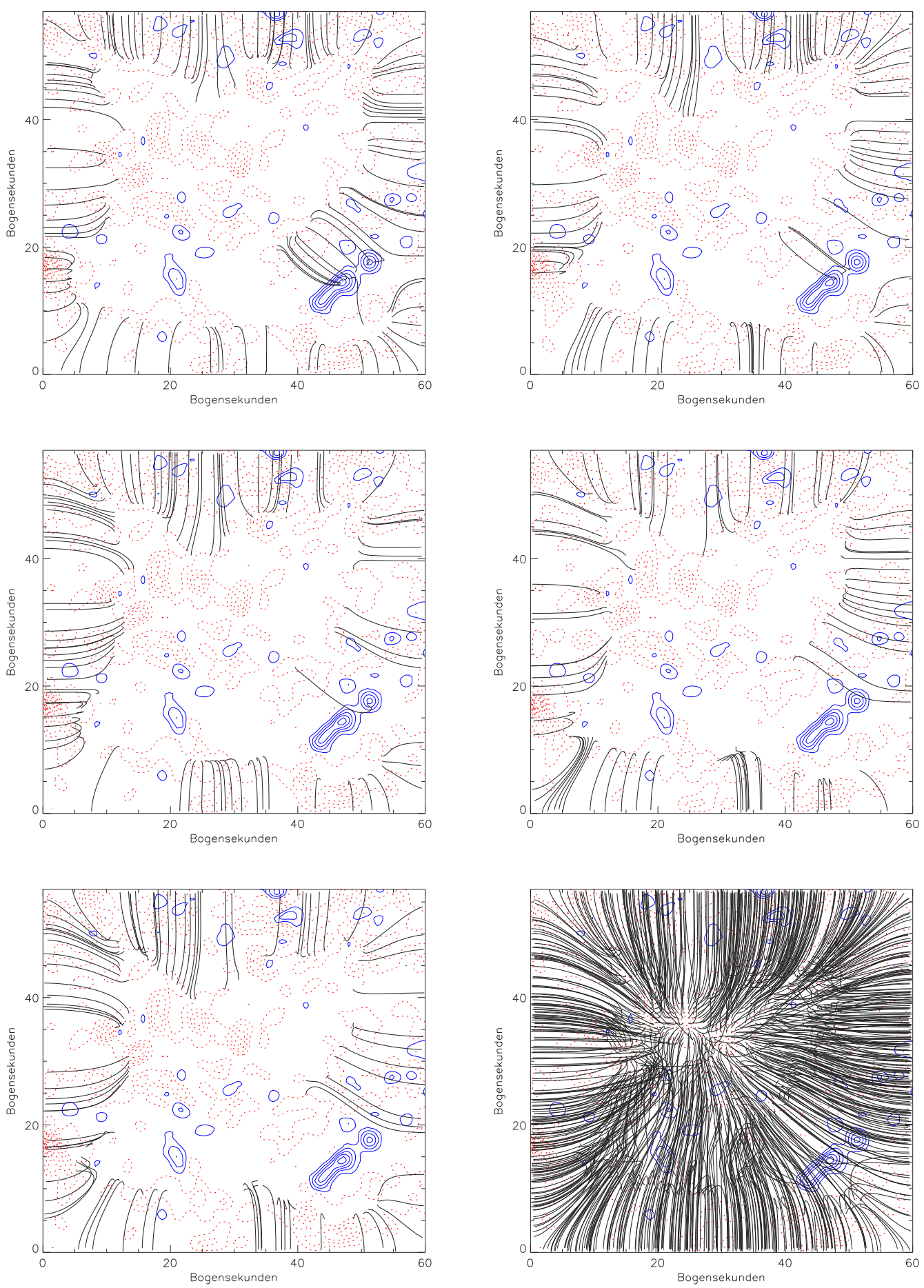

Abbildung 9.7: Fortsetzung Abb. 9.6 für extrapolierte Potentialfeldlinien die in Höhen $6000 \mathrm{~km}$ bis $7000 \mathrm{~km}, 7000 \mathrm{~km}$ bis $8000 \mathrm{~km}, 8000 \mathrm{~km}$ bis $9000 \mathrm{~km}$, $9000 \mathrm{~km}$ bis $10000 \mathrm{~km}, 10000 \mathrm{~km}$ bis $11000 \mathrm{~km}$ und $11000 \mathrm{~km}$ bis $68000 \mathrm{~km}$ reichen. 

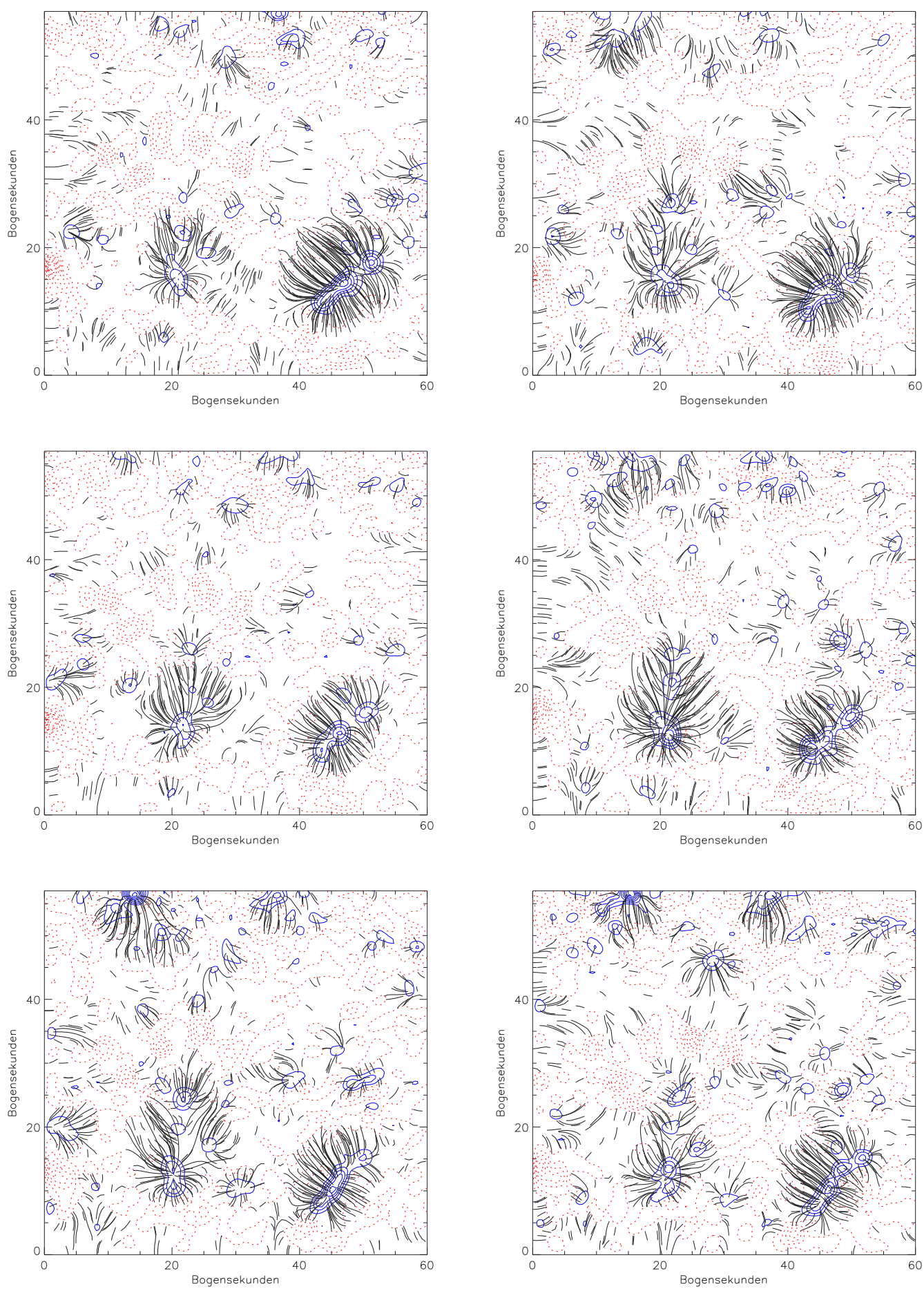

Abbildung 9.8: Von links nach rechts und oben nach unten sind die Ergebnisse der Extrapolationsrechnungen aus den Magnetogrammen Nr. 0 bis 5 (vgl. Abb. 8.1 bzw. 8.6) dargestellt. Es wurde hierbei der besseren Übersichtlichkeit halber nur jede zweite berechnete Potentialfeldlinie eingezeichnet. Weiterhin wurden ausschließlich geschlossene Feldlinien berücksichtigt und lokal offene weggelassen. 

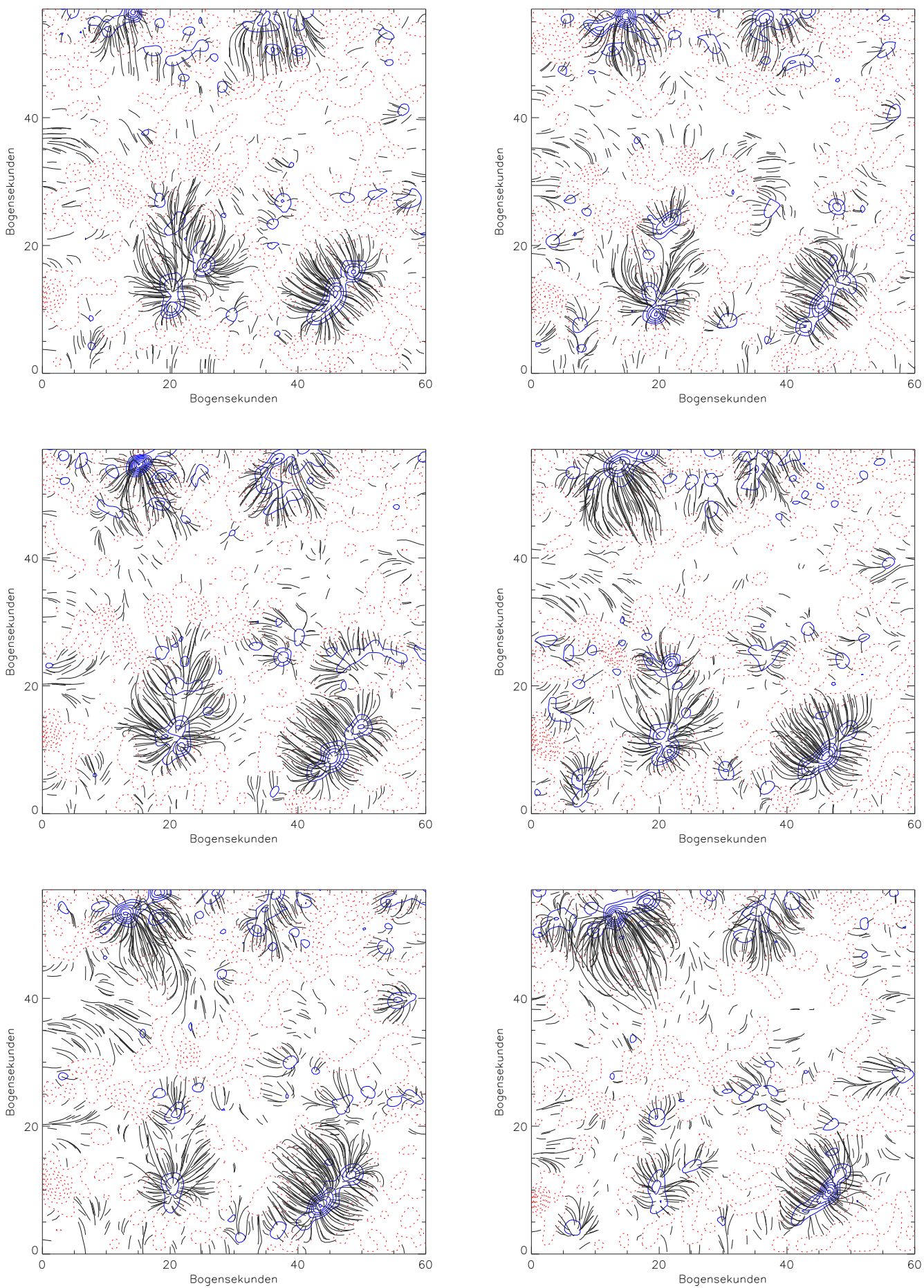

Abbildung 9.9: Fortsetzung Abb. 9.8, Ergebnisse aus den Magnetogrammen Nr. 6 bis 11. 

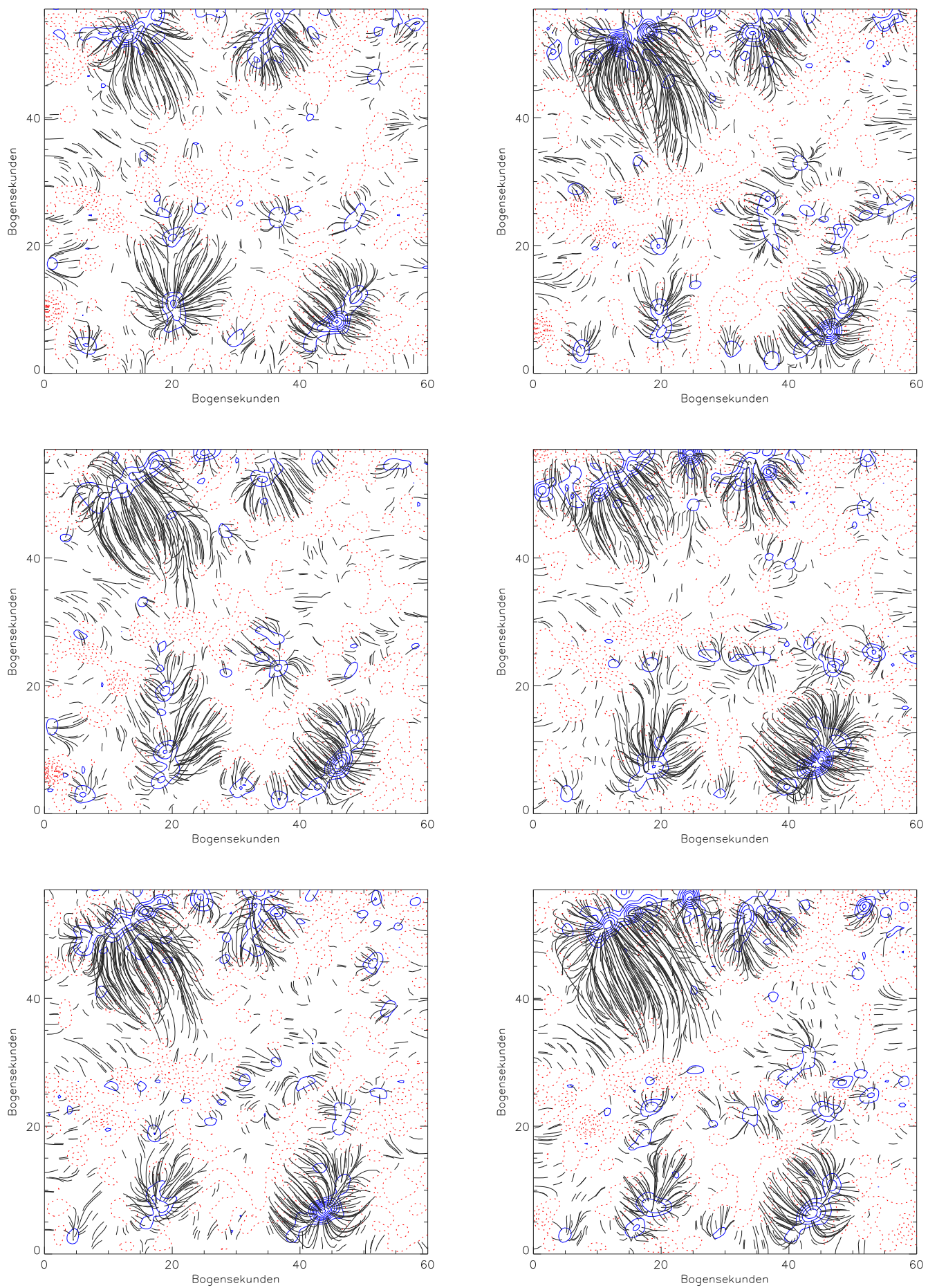

Abbildung 9.10: Fortsetzung Abb. 9.8, Ergebnisse aus den Magnetogrammen Nr. 12 bis 17. 

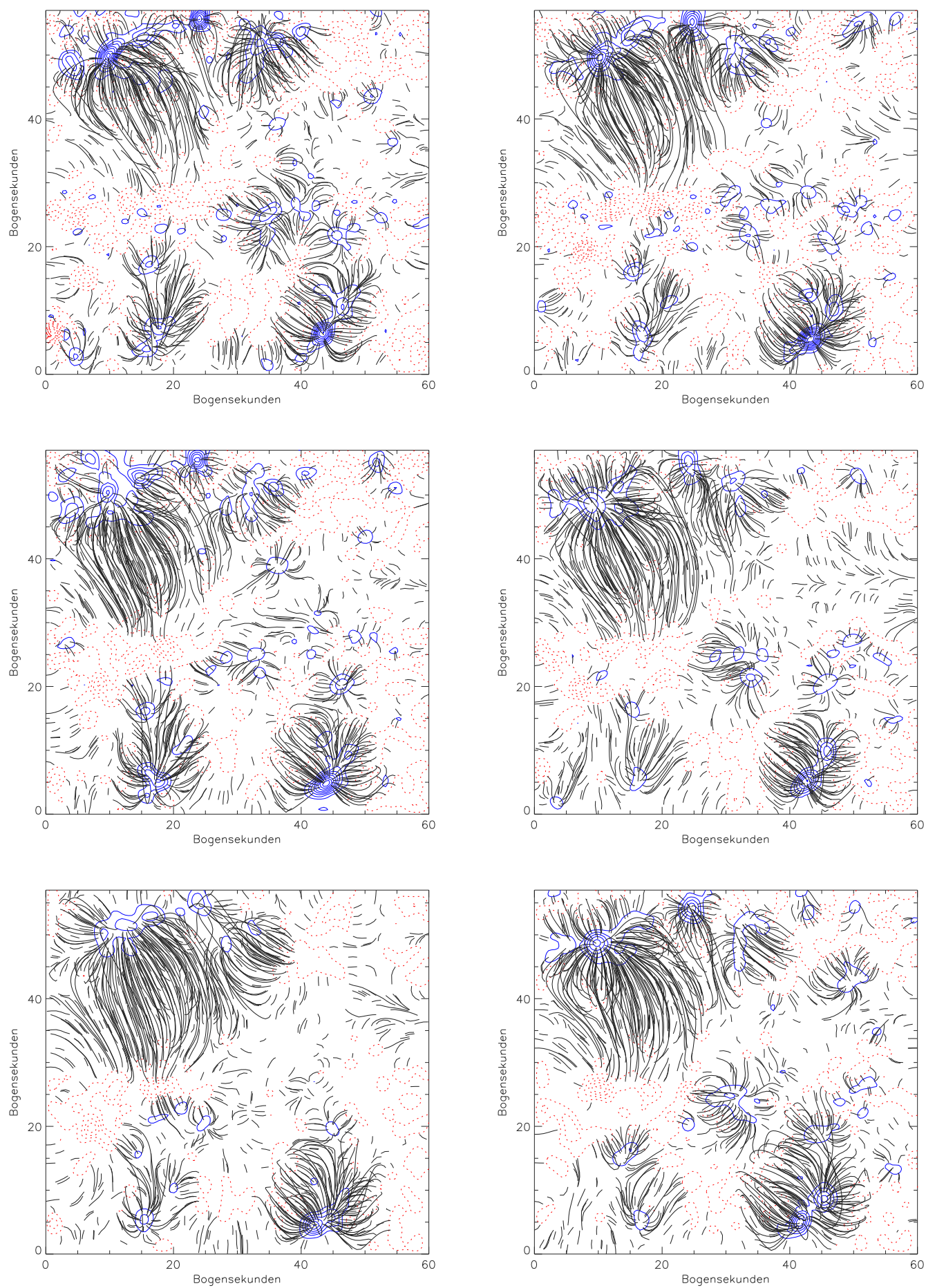

Abbildung 9.11: Fortsetzung Abb. 9.8, Ergebnisse aus den Magnetogrammen Nr. 18 bis 23. 

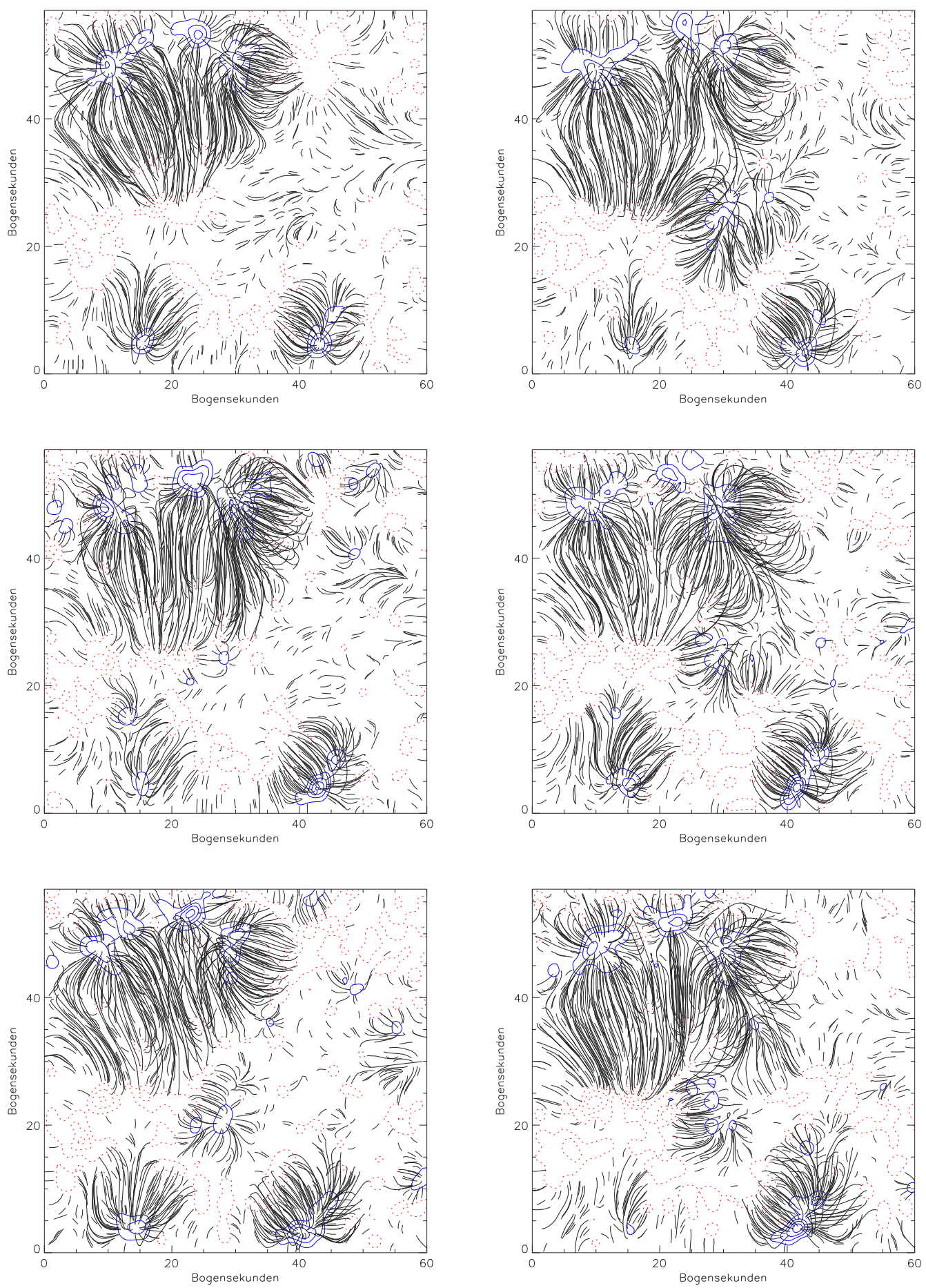

Abbildung 9.12: Fortsetzung Abb. 9.8, Ergebnisse aus den Magnetogrammen Nr. 24 bis 29. 

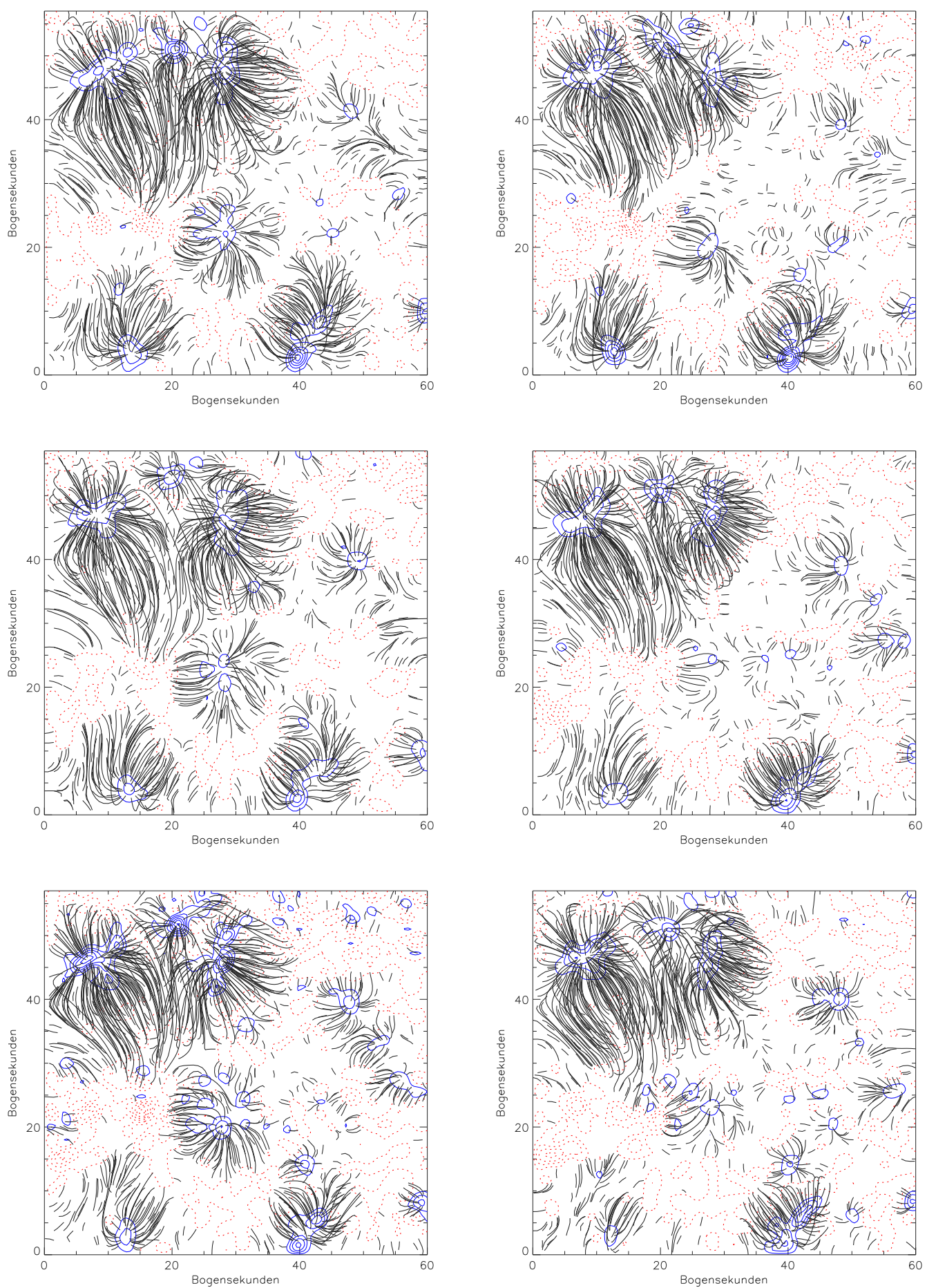

Abbildung 9.13: Fortsetzung Abb. 9.8, Ergebnisse aus den Magnetogrammen Nr. 30 bis 35. 

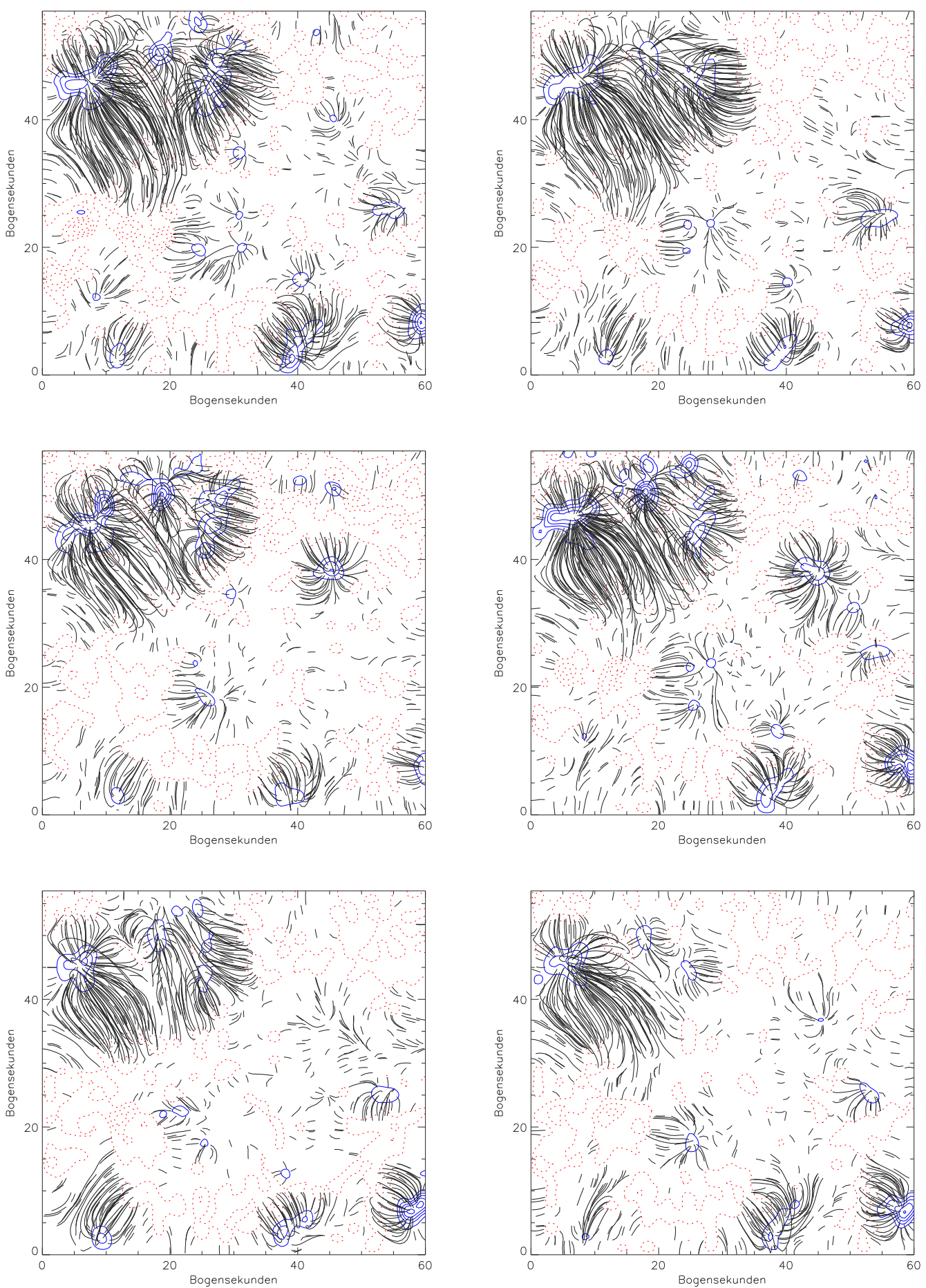

Abbildung 9.14: Fortsetzung Abb. 9.8, Ergebnisse aus den Magnetogrammen Nr. 36 bis 41. 

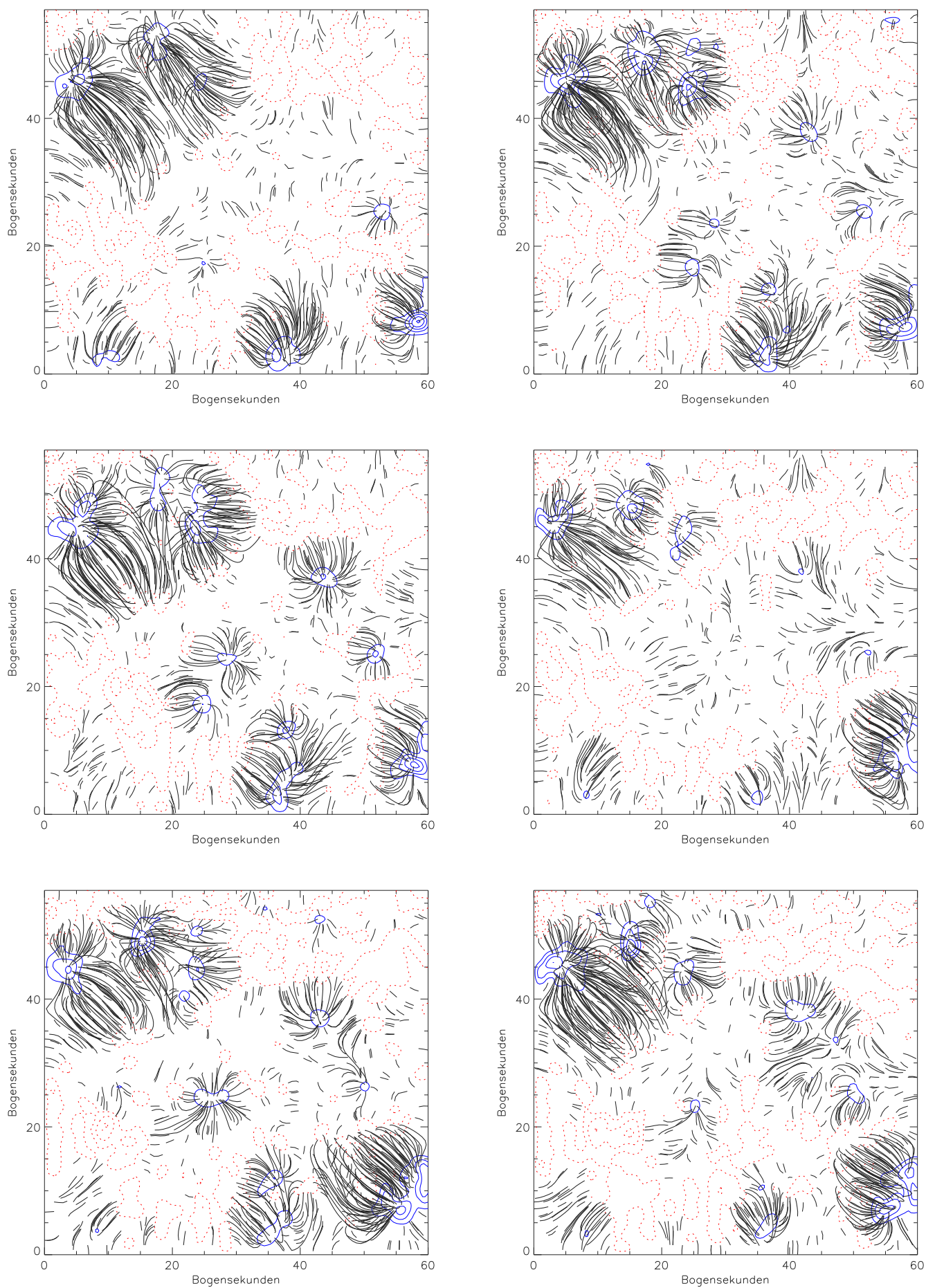

Abbildung 9.15: Fortsetzung Abb. 9.8, Ergebnisse aus den Magnetogrammen Nr. 42 bis 47. 


\section{Die $x t$-Diagramme}

Das aus der Serie der GCT-Magnetogramme (vgl. Kap. 8) an der Stelle der SUMERSpaltposition entnommene $x$-Diagramm ist in dem zeitlichen Rahmen der Beobachtungen des 10.5.1999 in Abb. 10.1 nocheinmal wiederholt (Abb. 8.10). Die Zeit verläuft in diesem und allen folgenden $x t$-Diagrammen von links nach rechts, während von unten nach oben die entsprechenden Ortspositionen aufgetragen sind. Die Einteilung der in Nord-Südrichtung (oben ist Norden) verlaufenden Ortsachse wurde in Einheiten von $5^{\prime \prime}$ vorgenommen. Die Zeitachse in Abb. 10.1 überstreicht das volle Intervall der GCTBeobachtungen von $15943 \mathrm{~s}$ (4h25 min 43s) und ist hier in Schritte von 10 Minuten unterteilt. Die Zeitachse beginnt um 8:09:13 UT und endet um 12:34:56 UT. Der mit den dicken Markierungen hervorgehobene Bereich von $10146 \mathrm{~s}$ (2 h 49 min 6s) entspricht dem Beobachtungszeitraum von SUMER. Er beginnt um 8:16:49 UT und endet um 11:05:55 UT. Während beim GCT auf 15943 s 120 Bilder entfallen, also im Mittel alle $133 \mathrm{~s}$ ein Bild entstand, hat SUMER alle $36 \mathrm{~s}\left(=\frac{10146 \mathrm{~s}}{284 \text { Aufnahmen }}\right)$ eine Aufnahme von je drei Spektren gemacht. In Abb. 10.1 setzen sich deutlich weiße Bereiche (positive Polarität) und schwarze Bereiche (negative Polarität) von dem grauen Hintergrund (Nullniveau) ab. Die Werte des magnetischen Flusses reichen hier von $-2,05 \cdot 10^{9} \mathrm{~Wb}$ bis $+3,41 \cdot 10^{9} \mathrm{~Wb}$. Das GCT-xt-Diagramm reißt um 9:54 UT (Bildnummer 46) abrupt ab, da hier der Beobachtungsbereich des GCTs die SUMER-Spaltposition nicht mehr einfängt (vgl. Kap. 8). Der Zeitraum synchroner Beobachtung beträgt somit $5844 \mathrm{~s}(1 \mathrm{~h} 37 \mathrm{~min} 24 \mathrm{~s})$ von 8:16:49 UT bis 9:54:13 UT.

In den folgenden $9 x t$-Diagrammen wird nur noch der gemeinsam beobachtete Bereich von $5844 \mathrm{~s}$ dargestellt. Der Abstand der Markierungen beträgt auf der Zeitachse 6 Minuten (= 10 mal SUMER-Intervallzeit). Die Ortsachse überstreicht ein Gebiet von 57", wobei aber aufgrund des gebogenen Verlaufs, bedingt durch die Bewegungskompensation (vgl. Kap. 8), 72" dargestellt werden. Auch hier wurde alle 5" eine Marke gesetzt.

Die Abbildung 10.2 zeigt nochmals das $x$-Diagramm des magnetischen Flusses, wobei sie jedoch zusätzlich mit Isokonturlinien bei $\pm 3 \cdot 10^{8} \mathrm{~Wb}, \pm 6 \cdot 10^{8} \mathrm{~Wb}, \pm 9 \cdot 10^{8} \mathrm{~Wb}, \pm 1.2$. $10^{9} \mathrm{~Wb}, \pm 1,5 \cdot 10^{9} \mathrm{~Wb}$ und $\pm 1,8 \cdot 10^{9} \mathrm{~Wb}$ versehen wurde.

Kurz unterhalb der Mitte des Bildes befindet sich ein Bereich, in dem negative Polarität zeitlich verfolgt wurde. Dieser Bereich bleibt über den ganzen Zeitraum von 5844 s bestehen. Die negative Polarität hat ein Extremum an magnetischem Fluß von $-2,05 \cdot 10^{9} \mathrm{~Wb}$. Obwohl hier im Mittel die negative Polarität bei weitem überwiegt, gibt es vereinzelte Gebiete positiver Polarität, die Stärken bis zu $4 \cdot 10^{8} \mathrm{~Wb}$ erreichen. Die drei markantesten 


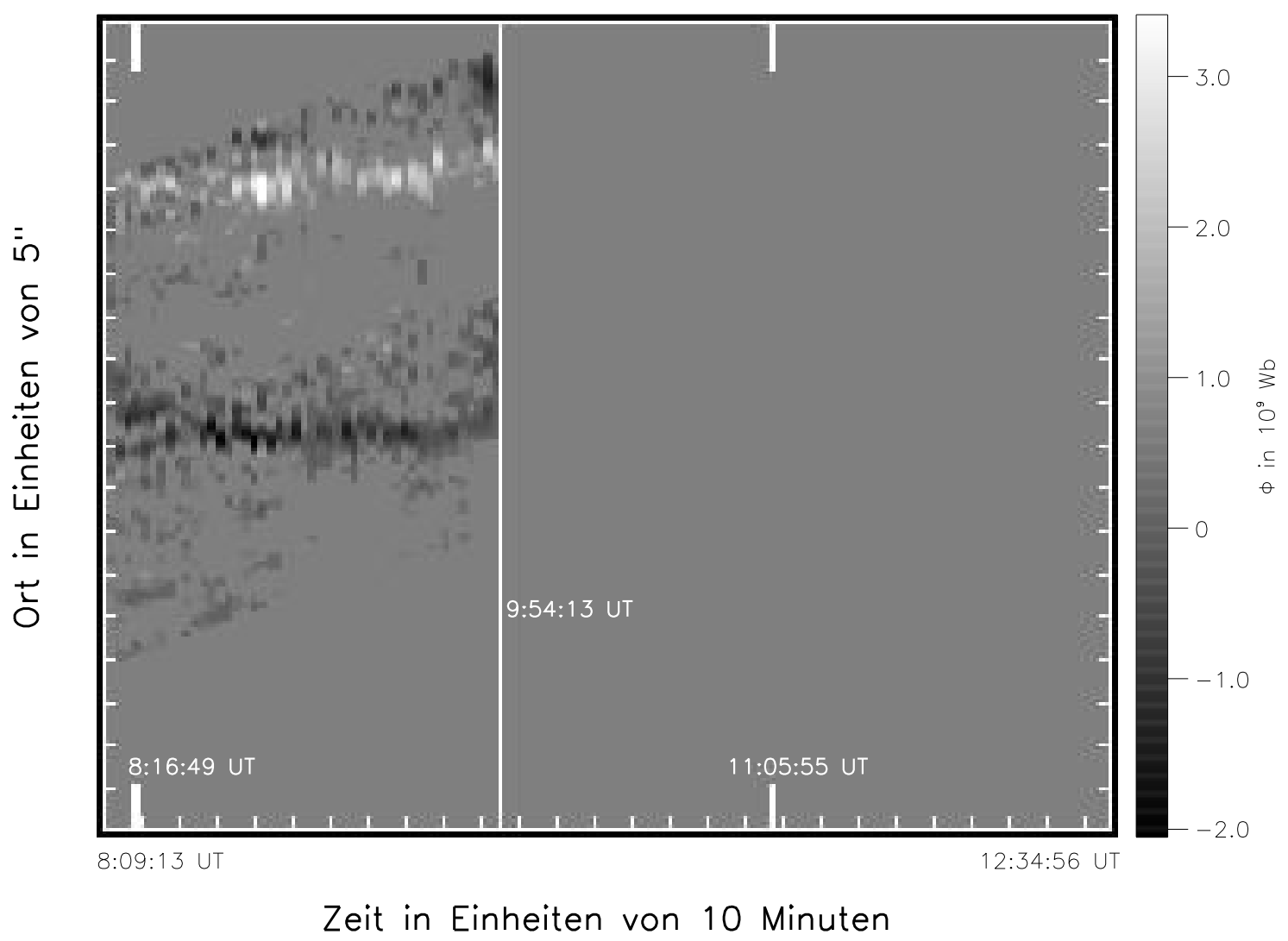

Abbildung 10.1: Zeitlicher Rahmen der Beobachtungen des 10.5.1999 mit magnetischen Flüssen im linken Teil. Die Zeitachse überstreicht das volle Intervall $\Delta t=15943 \mathrm{~s}(4 \mathrm{~h} 25 \mathrm{~min} 43 \mathrm{~s})$ der GCT-Beobachtungen von 8:09:13 UT bis 12:34:56 UT. Der mit den dicken Markierungen hervorgehobene Bereich von $10146 \mathrm{~s}$ ( 2 h 49 min $6 \mathrm{~s}$ ) entspricht dem Beobachtungszeitraum von SUMER (von 8:16:49 UT bis 11:05:55 UT). Das GCT- $x$-Diagramm reißt um 9:54:13 UT (Bildnummer 46) abrupt ab, da hier der Beobachtungsbereich des GCTs die SUMER-Spaltposition nicht mehr einfängt (vgl. Kap. 8). 


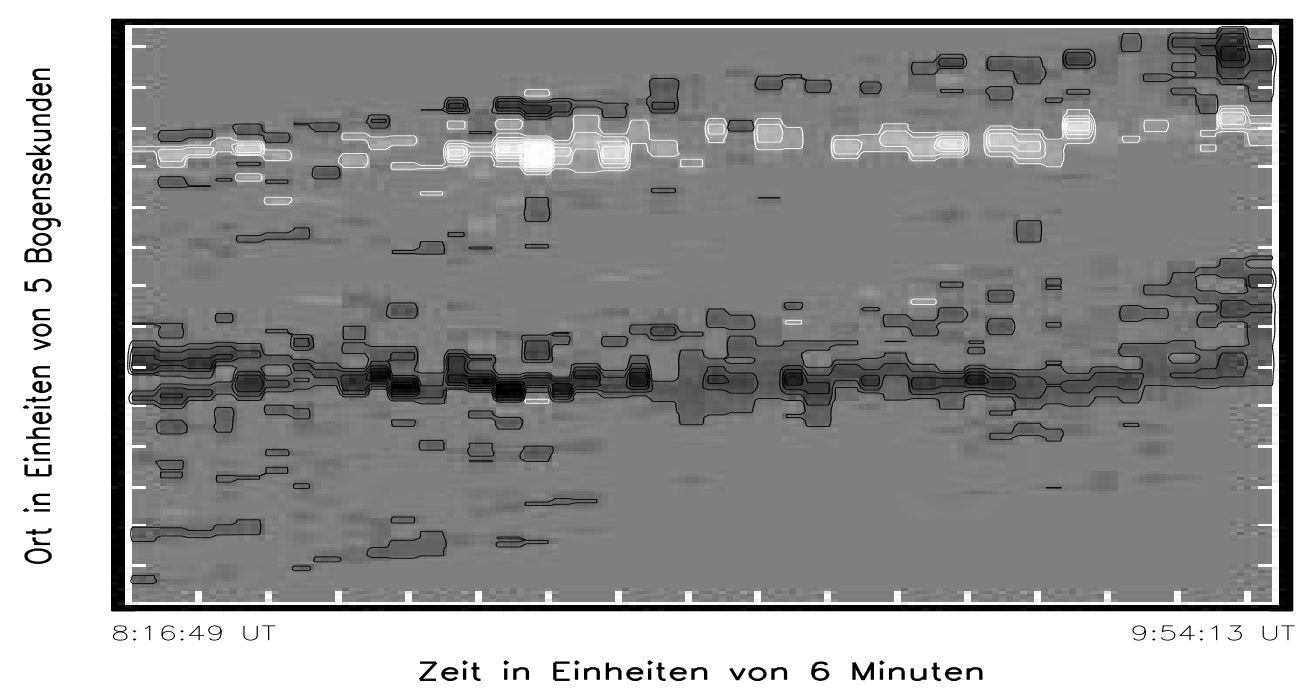

Abbildung 10.2: $x t$-Diagramm des magnetischen Flusses. Die weißen Bereiche (positive Polarität) und schwarzen Bereiche (negative Polarität) werden zusätzlich mit Isokonturlinien bei $\pm 3 \cdot 10^{8} \mathrm{~Wb}, \pm 6 \cdot 10^{8} \mathrm{~Wb}, \pm 9 \cdot 10^{8} \mathrm{~Wb}, \pm 1.2$. $10^{9} \mathrm{~Wb}, \pm 1,5 \cdot 10^{9} \mathrm{~Wb}$ und $\pm 1,8 \cdot 10^{9} \mathrm{~Wb}$ vom grauen Hintergrund (Nullniveau) abgehoben. Die Werte des magnetischen Flusses reichen von $-2,05 \cdot 10^{9} \mathrm{~Wb}$ bis $+3,41 \cdot 10^{9} \mathrm{~Wb}$. 


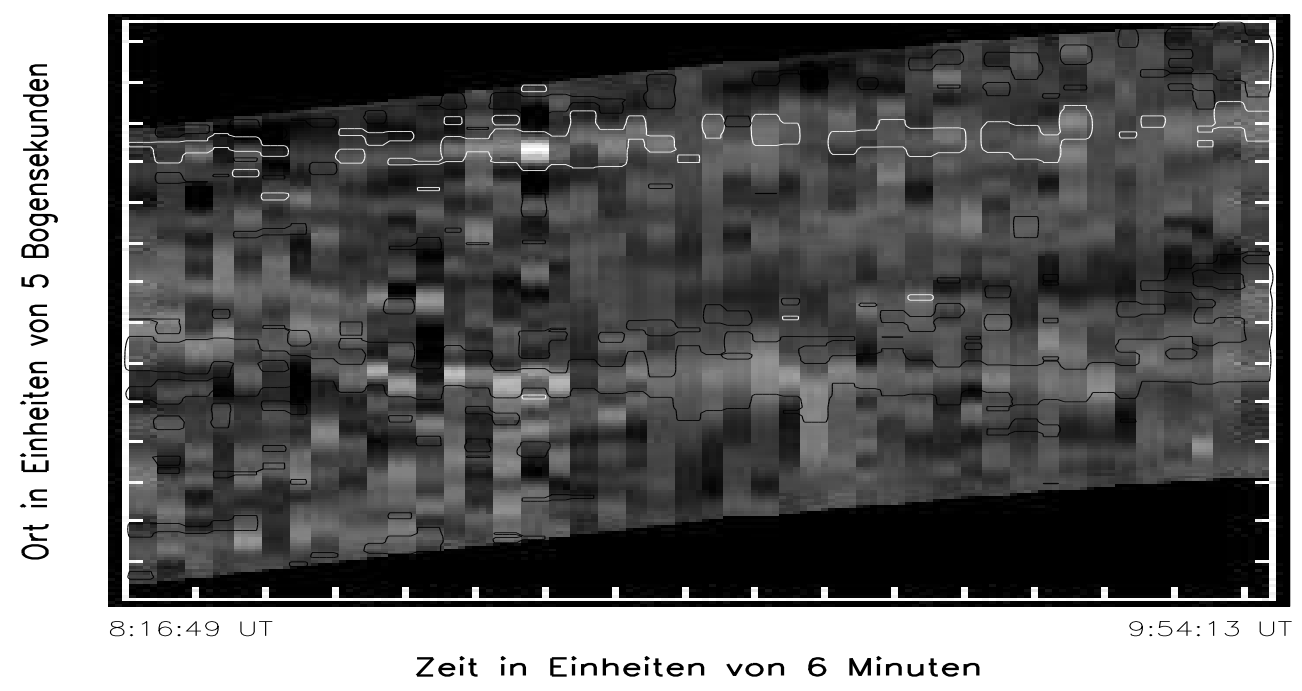

Abbildung 10.3: $x$-Diagramm der aus dem Zentrum der Fe I-Linie gewonnenen Intensitätsverteilung. Das Maximum der Intensität befindet sich an der Stelle des Maximums des magnetischen Flusses der positiven Polarität.

tauchen in unmittelbarer Nähe der negativen Polarität auf und verschwinden nach ca. 2 Minuten wieder (um ca. 8:51 UT bei $\sim 25^{\prime \prime}$, um ca. 9:13 UT bei $\sim 35^{\prime \prime}$ und um ca. 9:24 UT bei $\sim 39^{\prime \prime}$ nördlich von der unteren Kante des Bildes (Abb. 10.2); der magnetische Fluß erreicht hier Werte von $3 \cdot 10^{8} \mathrm{~Wb}$ bis $4 \cdot 10^{8} \mathrm{~Wb}$ ). Im oberen Teil des Diagramms bewegt sich ein Gebiet positiver Polarität in ganz ähnlicher Weise wie das untere negative durch die Zeit. Anders als das untere jedoch, verschwindet es immer wieder für Zeiträume von der Größenordnung von 2 Minuten, um dann wieder an der selben Stelle aufzutauchen. Nach etwa 34 min erreicht das Gebiet ein deutliches Maximum des magnetischen Flusses von $3,4 \cdot 10^{9} \mathrm{~Wb}$ für ca. 2 Minuten; der Fluß fällt dann, ähnlich rasant wie er sich aufgebaut hat, wieder ab. Die gesamte Erscheinung dauert etwa $15 \mathrm{~min}$.

Den folgenden 8 Diagrammen ist die unterste Isokonturlinie des Magnetogramms bei $\pm 3 \cdot 10^{8} \mathrm{~Wb}$ unterlegt.

Das erste Bild (Abb. 10.3) dieser Serie von Kompositdarstellungen zeigt das aus dem Zentrum der Fe I-Linie erzeugte Intensitätsbild. Die Aufhellungen liegen zumeist in Gebieten magnetischer Aktivität. Insbesondere liegt das Maximum der Intensität an der Stelle des Maximums des magnetischen Flusses der positiven Polarität.

Die folgende Darstellung (Abb. 10.4) zeigt das aus den Ca II $K$-Filtergrammen gewonnene $x t$-Diagramm. Auch hier folgen die Aufhellungen dem Verlauf des magnetischen Flusses. Und wieder zeigt sich insbesondere dort eine starke Aufhellung, wo auch der magnetische Fluß der positiven Polarität ein Maximum hat. Die vertikalen Striche sind Datenlücken. Der horizontale Strich stammt von einem Staubkorn vor dem Detektor, das sich zunächst ein bißchen bewegt hat und dann liegen geblieben ist. 


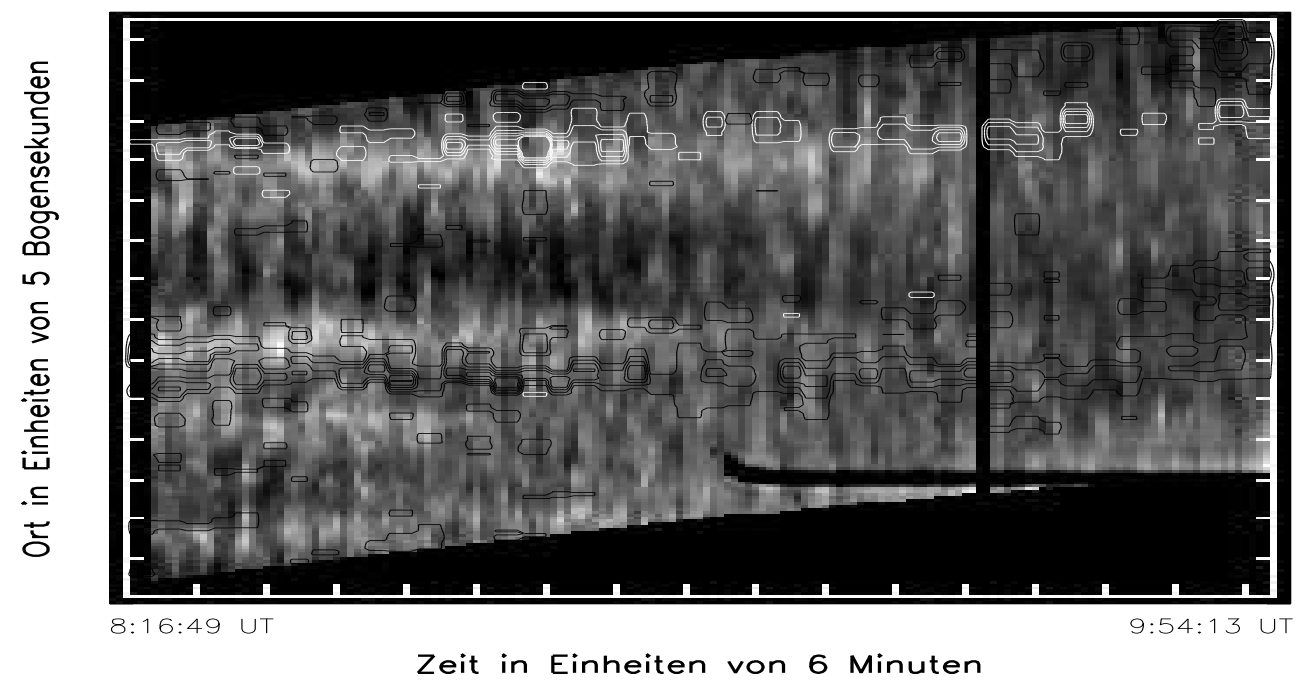

Abbildung 10.4: Aus den Ca II $K$-Filtergrammen erzeugtes $x$-Diagramm. Die Aufhellungen folgen im wesentlichen dem Verlauf des magnetischen Flusses. Die vertikalen Striche sind Datenlücken. Der horizontale Strich unten rechts stammt von einem Staubkorn vor dem Detektor, das sich zunächst ein bißchen bewegt hat und dann liegen geblieben ist.

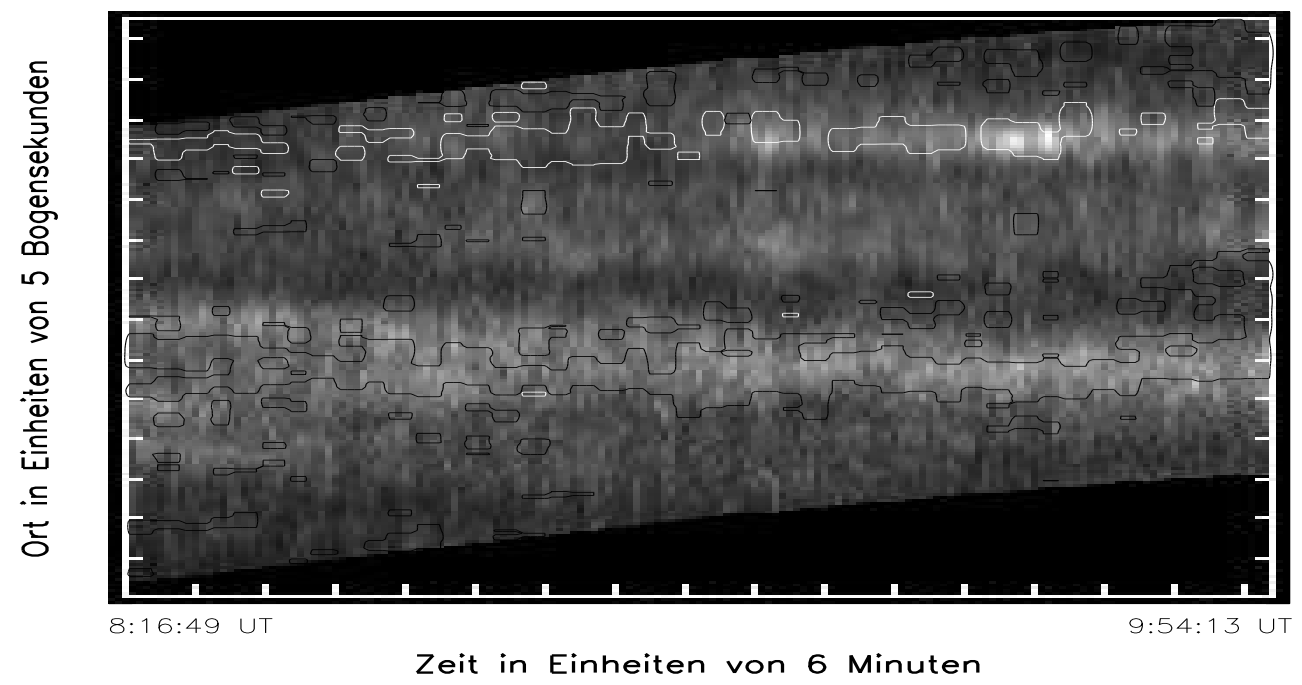

Abbildung 10.5: $x$-Diagramm aus den spektral integrierten Spektren der C I-Linie. 


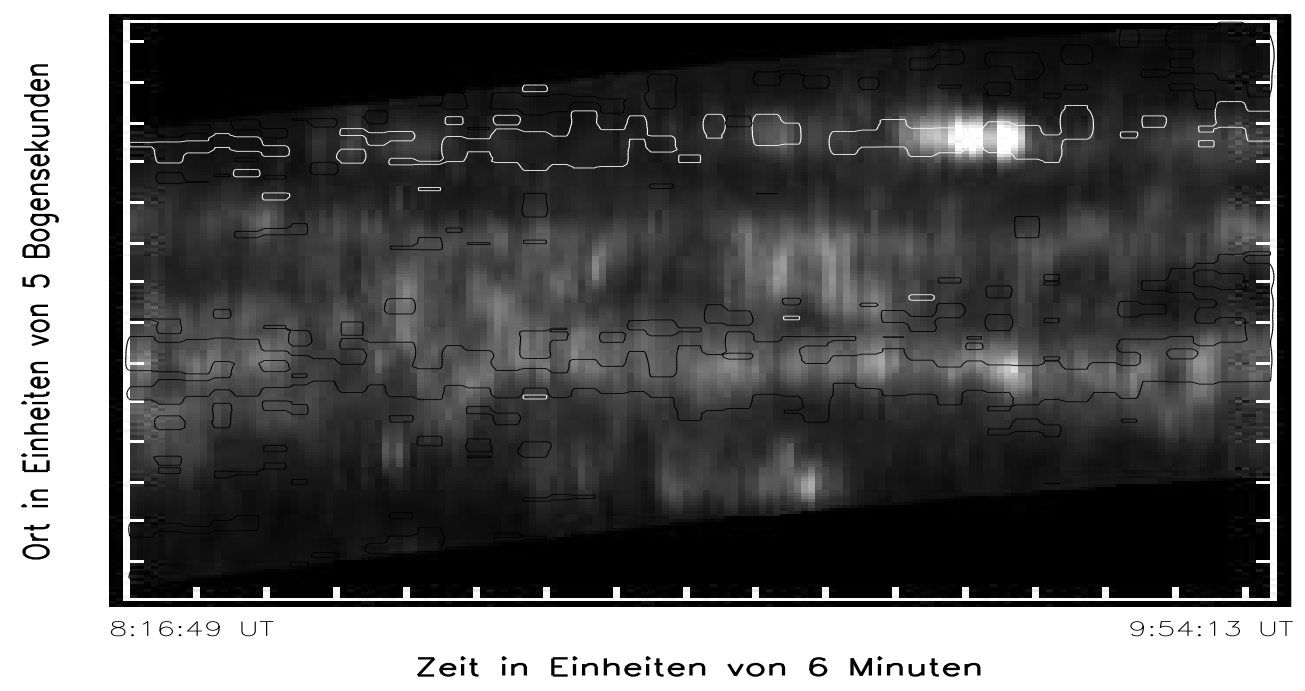

Abbildung 10.6: $x t$-Diagramm aus den spektral integrierten Spektren der C IV-Linie.

Die Abb. 10.5 zeigt das $x$-Kompositdiagramm der C I-Linie. Im unteren Teil des Bildes steht die Aufhellung in guter Übereinstimmung mit dem dort vorherrschenden Magnetfeld negativer Polarität. In der Mitte des Bildes entwickelt sich eine Aufhellung bis zu einem lokalen Maximum und klingt wieder ab, ohne daß ein magnetisches Feld gemessen wird. In dem oberen Teil des Diagramms findet sich zu Beginn bis über das Maximum des magnetischen Flusses positiver Polarität keine Korrelation zwischen Aufhellung und Magnetfeld. Dann entsteht zunächst eine kleine Aufhellung, die wieder abfällt, sich jedoch nach ca. 16 Minuten zu einer starken, das Maximum des Diagramms darstellenden, Aufhellung entwickelt. Dieser Teil wird von einem Magnetfeld begleitet.

Abb. 10.6 zeigt das Diagramm der C IV-Linie. Im unteren und mittleren Bereich sind Aufhellungen mittlerer Stärke zu erkennen, die zwar zum Teil in dem Gebiet der negativen Polarität liegen, aber zum Teil eben auch weit ober- und unterhalb örtlich versetzt davon. Die Aufhellungen im mittleren Bereich des Diagramms sind gekennzeichnet von größeren Phasen der Unterbrechung. In den Bereich positiver Polarität fällt, neben einigen unsteten kleinen Aufhellungen, auch einmal für ca. 12 Minuten eine, allerdings extrem starke, Aufhellung.

Das folgende $x$-Diagramm (Abb. 10.7) der Ne viII-Linie hat eine dem C IV-Diagramm ähnliche Erscheinung, jedoch unterliegt das Aufhellungsmuster nicht so starken Schwankungen und weist insbesondere nicht eine so extreme Aufhellung im Bereich der positiven Polarität auf.

Die Abb. 10.8 zeigt das $x t$-Diagramm aus den Dopplergrammen der Fe I-Linie. Helle Bereiche sind Bereiche in denen aufgrund von Rotverschiebungen der Linie auf positive Geschwindigkeiten geschlossen wurde. Sie weisen also auf Materiebewegungen hin, die von dem Beobachter weg und zur Sonne hin zeigen. Bei den dunklen Bereichen ist es 


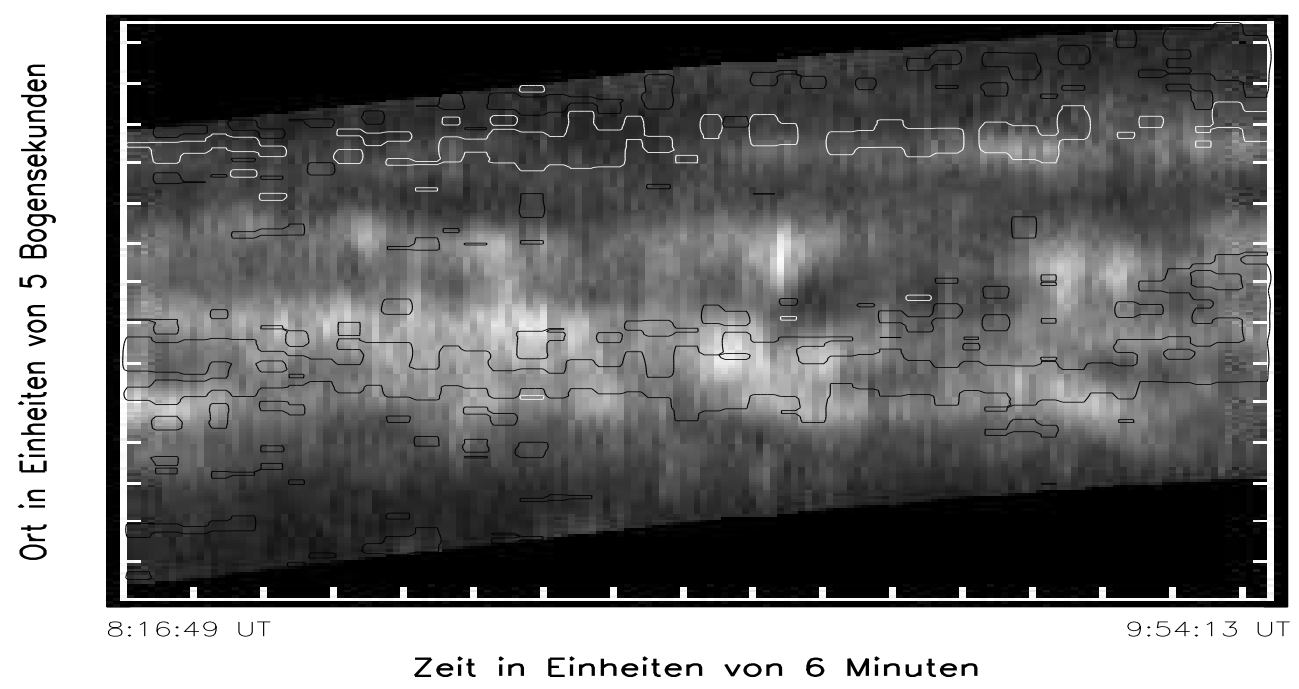

Abbildung 10.7: xt-Diagramm aus den spektral integrierten Spektren der Ne VIII-Linie.

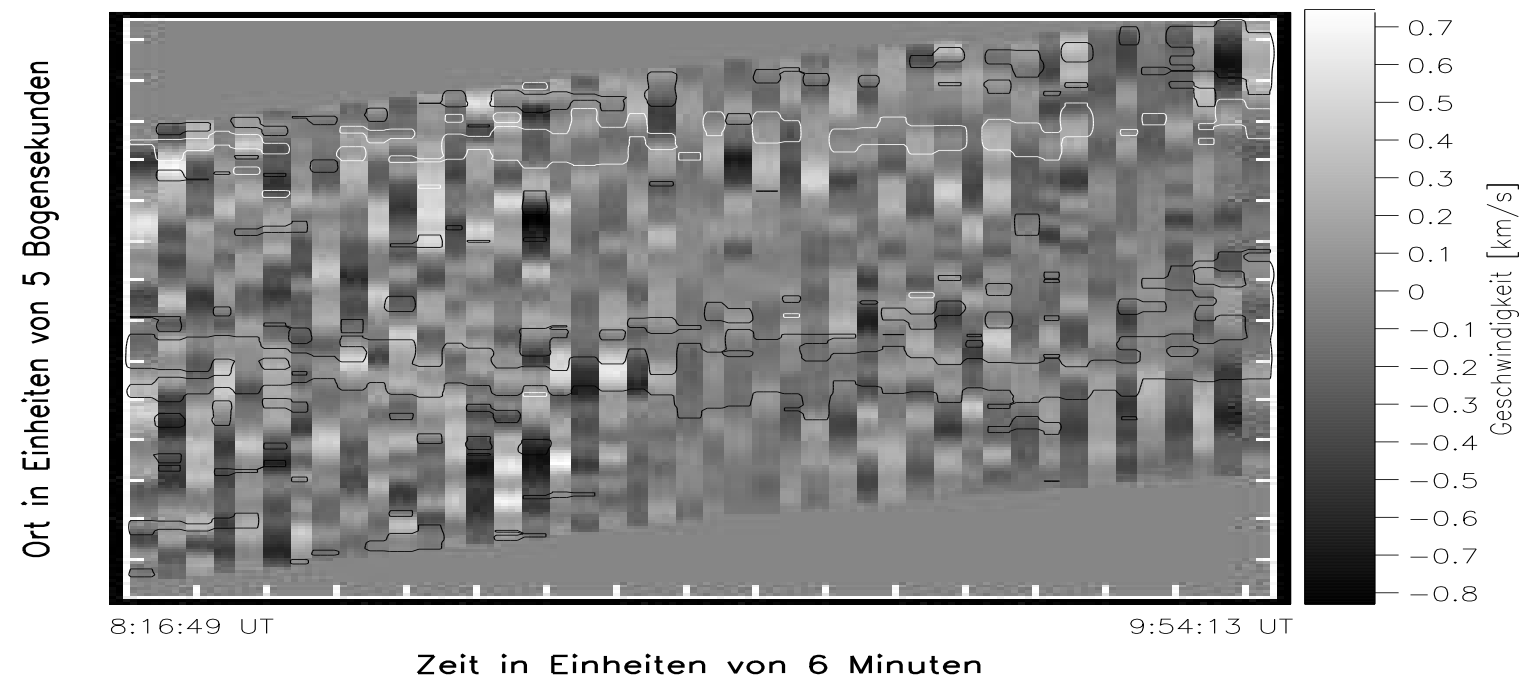

Abbildung 10.8: $x$-Diagramm aus den Dopplergrammen der Fe I-Linie (aus dem ISignal). 


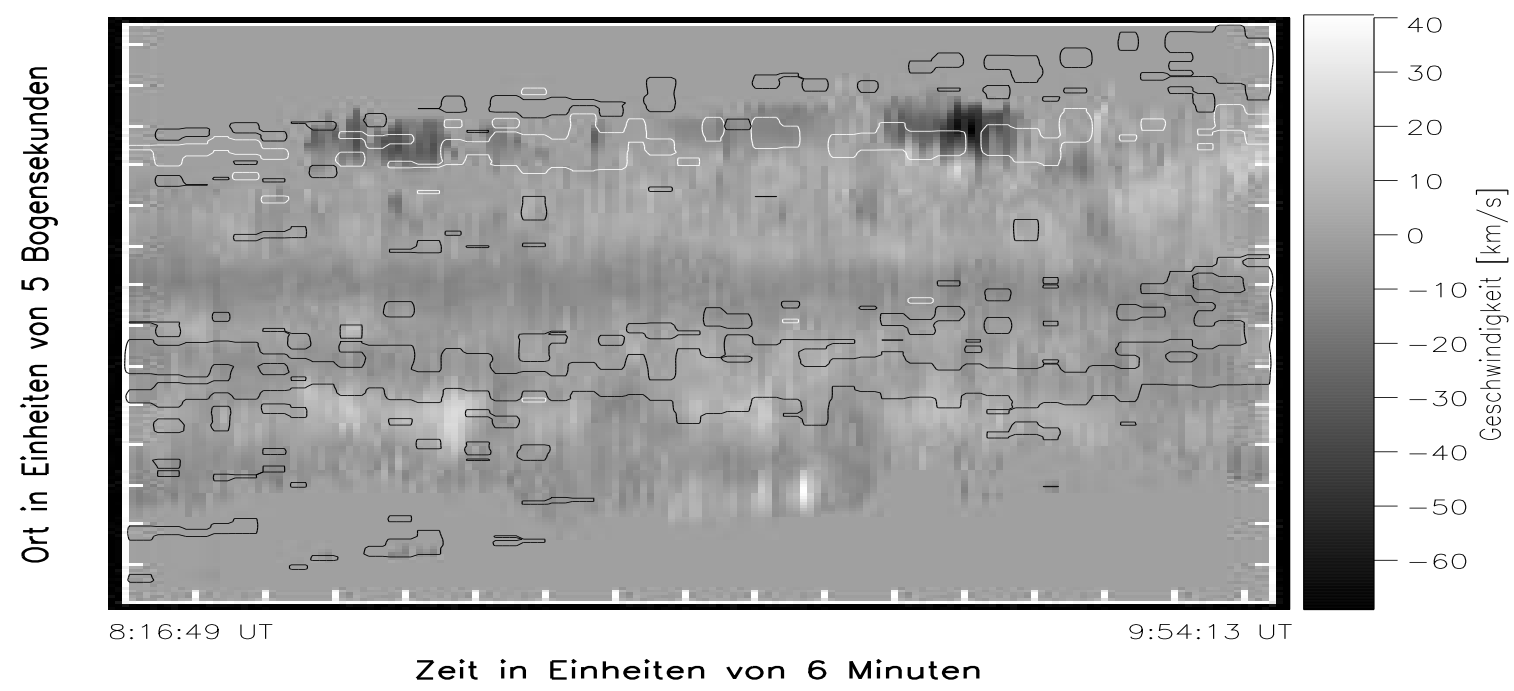

Abbildung 10.9: $x t$-Dopplergramm aus der C IV-Linie.

entsprechend umgekehrt. Die Geschwindigkeiten reichen bei dem aus dem I-Signal gewonnenen Dopplergramm von etwa $-800 \mathrm{~m} / \mathrm{s}$ bis $+700 \mathrm{~m} / \mathrm{s}$. Eine Korrelation mit der zeitlichen Entwicklung des magnetischen Flusses kann nicht festgestellt werden.

Das in Abb. 10.9 gezeigte Dopplergramm aus der C IV-Linie weist Geschwindigkeiten von bis zu $-69 \mathrm{~km} / \mathrm{s}$ und bis $+40 \mathrm{~km} / \mathrm{s}$ auf. Die meiste Zeit jedoch liegen die Geschwindigkeiten um $\pm 10 \mathrm{~km} / \mathrm{s}$. Der große dunkle Bereich oben rechts im Diagramm ist das Gebiet der stärksten Aufwärtsströmung. Es liegt in dem Bereich, in dem auch das C IVIntensitätsbild eine maximale Aufhellung zeigt. Jedoch ist gerade an dieser Stelle das Diagramm fragwürdig, da sich die Emissionslinie hier in zwei Komponenten teilt. Die Verhältnisse werden durch das Anpassen nur einer Gaußfunktion inkorrekt wiedergegeben, dazu mehr in Kapitel 10.

In dem $x t$-Dopplergramm Abb. 10.10, gewonnen aus der NevinI-Linie, finden sich Aufwärtsströmungen von bis zu $40 \mathrm{~km} / \mathrm{s}$ und Abwärtsströmungen bis zu knapp $38 \mathrm{~km} / \mathrm{s}$. Im größten Teil des Diagramms pendeln die Werte der Geschwindigkeit zwischen $+5 \mathrm{~km} / \mathrm{s}$ und $-20 \mathrm{~km} / \mathrm{s}$.

Die für den Doppler-Effekt relevanten Verschiebungen wurden durch anpassen von Gaußfunktionen an die Emissionslinienprofile der C IV- bzw. Ne VIII-Linie gefunden.

Abbildung 10.11 zeigt die $x t$-Diagramme (von links nach rechts) des magnetischen Flusses und der spektral integrierten Intensität der C IV- bzw. Ne viII-Linie. Es sind, durch horizontale Linien, zum einen der Bereich überwiegend positiver Polarität (weiße Bereiche) und zum anderen der Bereich vornehmlich negativer Polarität (schwarze Bereiche) eingegrenzt. Diese Bereiche wurden in Ortsrichtung über $11^{\prime \prime}$ bzw. 16" gemittelt und gegen die Zeit aufgetragen (vgl. Abb. 10.12 und 10.13). Hierbei wurde negative und positive Polarität des magnetischen Flusses getrennt behandelt. 


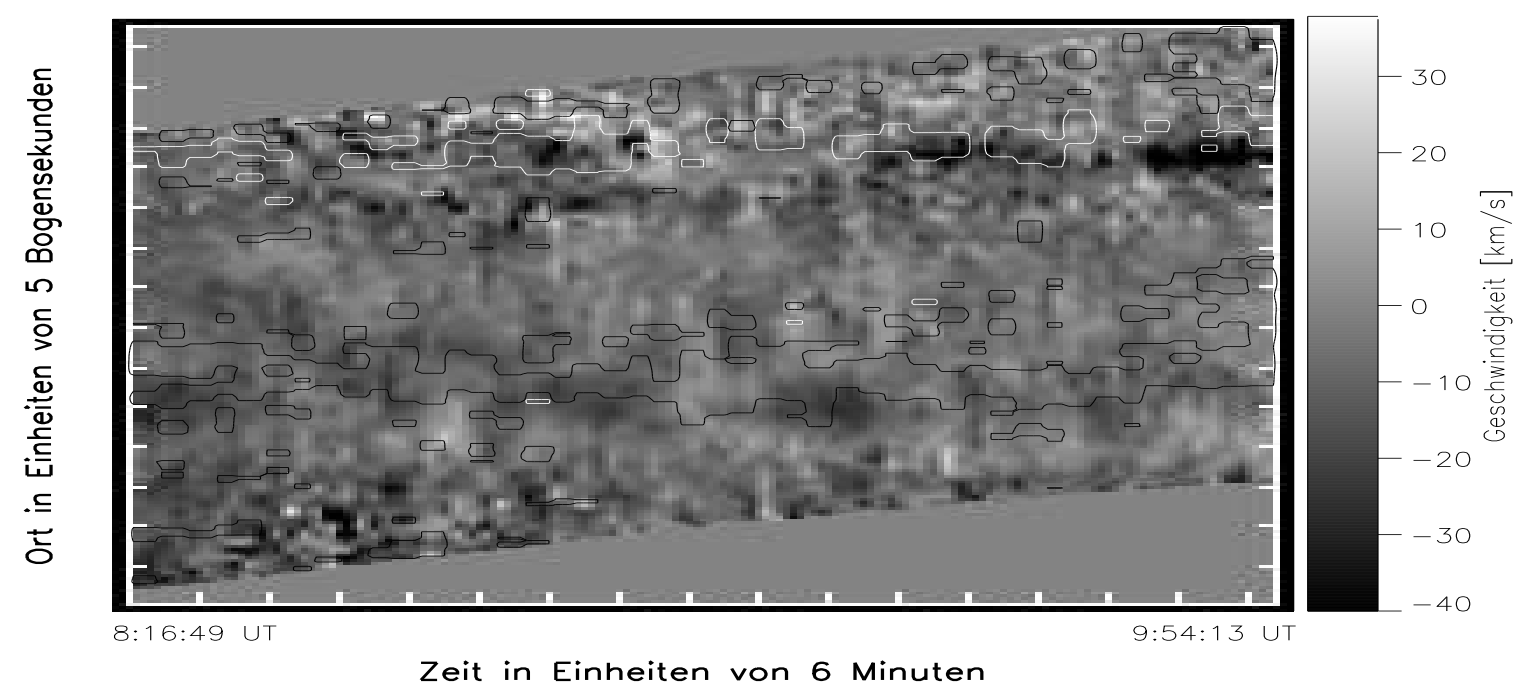

Abbildung 10.10: $x t$-Dopplergramm aus der Ne VIII-Linie.
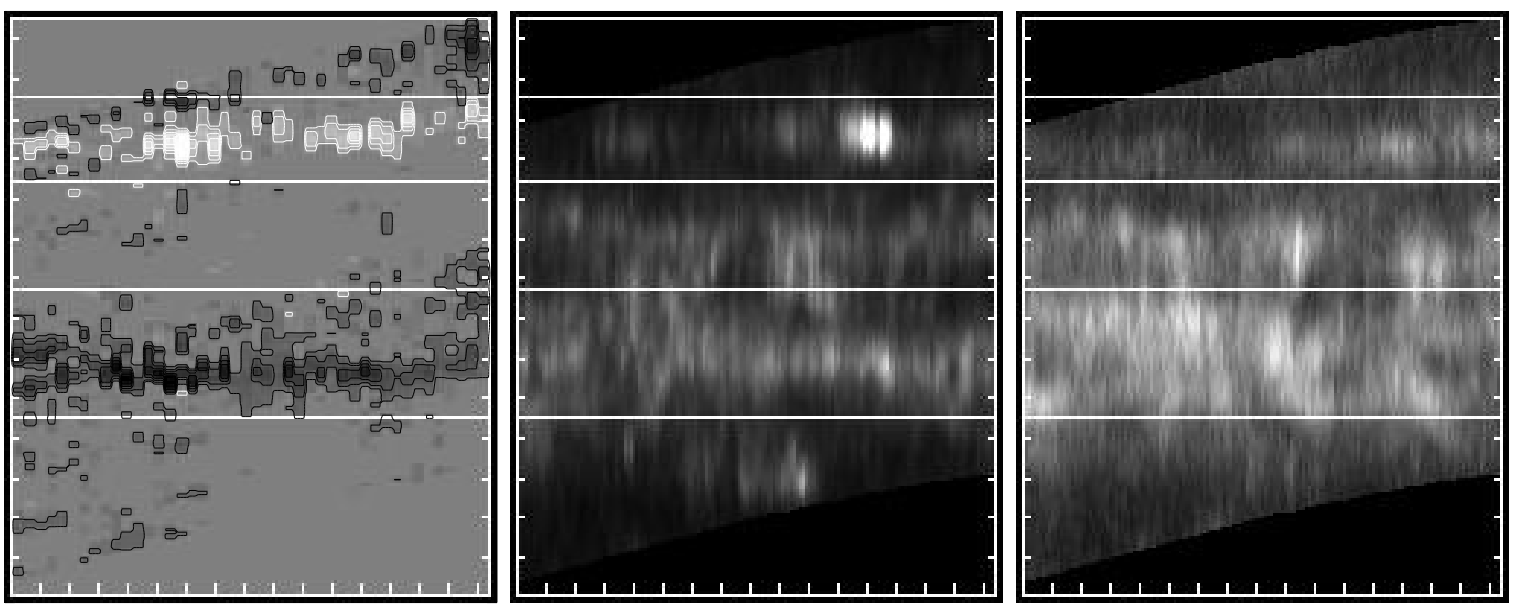

Abbildung 10.11: xt-Diagramme (von links nach rechts) des magnetischen Flusses, der spektral integrierten Intensität der C IV- und Ne VIII-Linie. Es sind, durch horizontale Linien, zum einen der Bereich überwiegender positiver Polarität (weiße Bereiche) und zum anderen der Bereich vornehmlich negativer Polarität (schwarze Bereiche) eingegrenzt. 


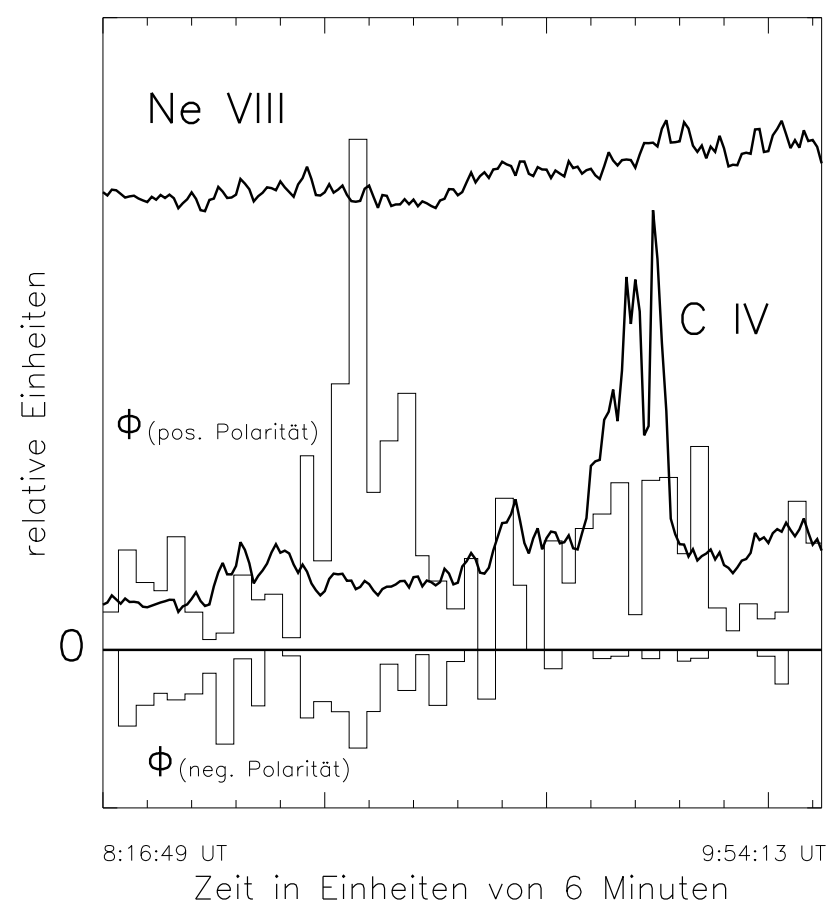

Abbildung 10.12: Über den Ortsbereich von 11" des oberen in Abb. 10.11 markierten Bereichs gemittelte Intensität des Ne VIII- bzw. C IV-Spektrogramms in Abhängigkeit von der Zeit. Weiterhin ist der entsprechend gemittelte magnetische Fluß getrennt nach Polarität aufgetragen. Der besseren Übersichtlichkeit wegen ist der Verlauf der Intensität der Ne VIII-Linie gegen den der C IV-Linie verschoben aufgetragen. 


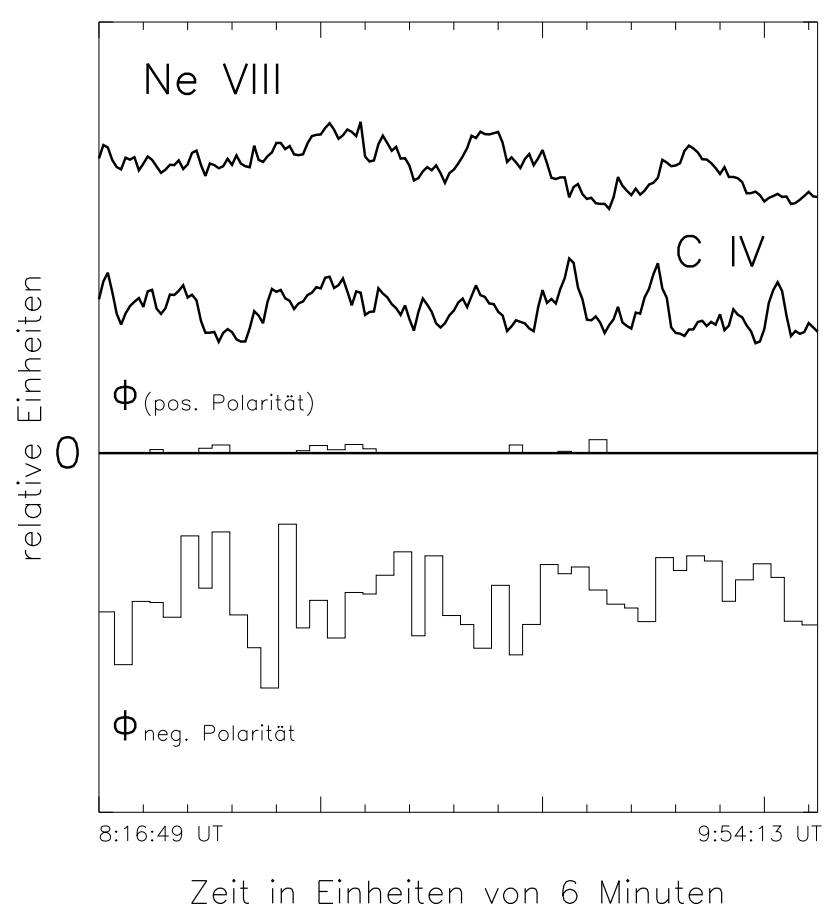

Abbildung 10.13: Über den Ortsbereich von 16" des unteren, in Abb. 10.11 markierten Bereichs gemittelte Intensität des Ne viII- bzw. C IV-Spektrogramms in Abhängigkeit von der Zeit. Getrennt nach Polarität ist ferner der entsprechend gemittelte magnetische Fluß aufgetragen. Der besseren Übersichtlichkeit halber ist der Verlauf der Intensität der Ne VIII-Linie gegen den der C IV-Linie verschoben aufgetragen.

Der in Abb. 10.12 aufgetragene Verlauf der Intensität der Ne VIII-Linie aus dem Bereich vornehmlich positiver Polarität hat, sieht man zum einen von kleineren Abweichungen und zum anderen vor allem von der starken Aufhellung der C IV-Linie ab, einige Ähnlichkeit mit dem Verlauf der Intensität der C IV-Linie. Der magnetische Fluß positiver Polarität hat ein Maximum etwa $\frac{1}{2} \mathrm{~h}$ bevor die CIV-Linie ihre maximale Aufhellung erreicht. Während des Zeitraumes der maximalen Aufhellung der C IV-Linie mit einer Dauer von ca. 12 Minuten verschwindet der magnetische Fluß negativer Polarität fast komplett. Der magnetische Fluß positiver Polarität hält sich in diesem Zeitraum auf einem mittleren Niveau, mit einer Unterbrechung nach Ablauf etwa der halben Zeit der Aufhellung. Der Intensitätsverlauf der C IV-Linie hat eine, allerdings zeitlich um ca. 1 Minute verschobene Entsprechung dazu. Mit dem Abklingen der Aufhellung sackt auch der magnetische Fluß positiver Polarität auf ein lokales Minimum. In Abb. 10.13 sind in Analogie zu Abb. 10.12 die Verläufe der Intensitäten bzw. des magnetischen Flusses aus 
dem unteren, in Abb. 10.11 markierten Bereich dargestellt. Während im dargestellten Zeitraum die Intensität der Ne vinI-Linie drei lange, flache und von kurzzeitigen Fluktuationen überlagerte Aufhellungen von jeweils etwa 18 Minuten aufweist, fluktuiert die Intensität der C IV-Linie etwas heftiger in Intervallen von etwa einem drittel dieser Zeit. Der Bereich wird durchgängig von magnetischem Fluß negativer Polarität dominiert. Dieser Fluß weist auf relativ hohem Niveau Schwankungen von 6 min bis 10 min Dauer auf. 


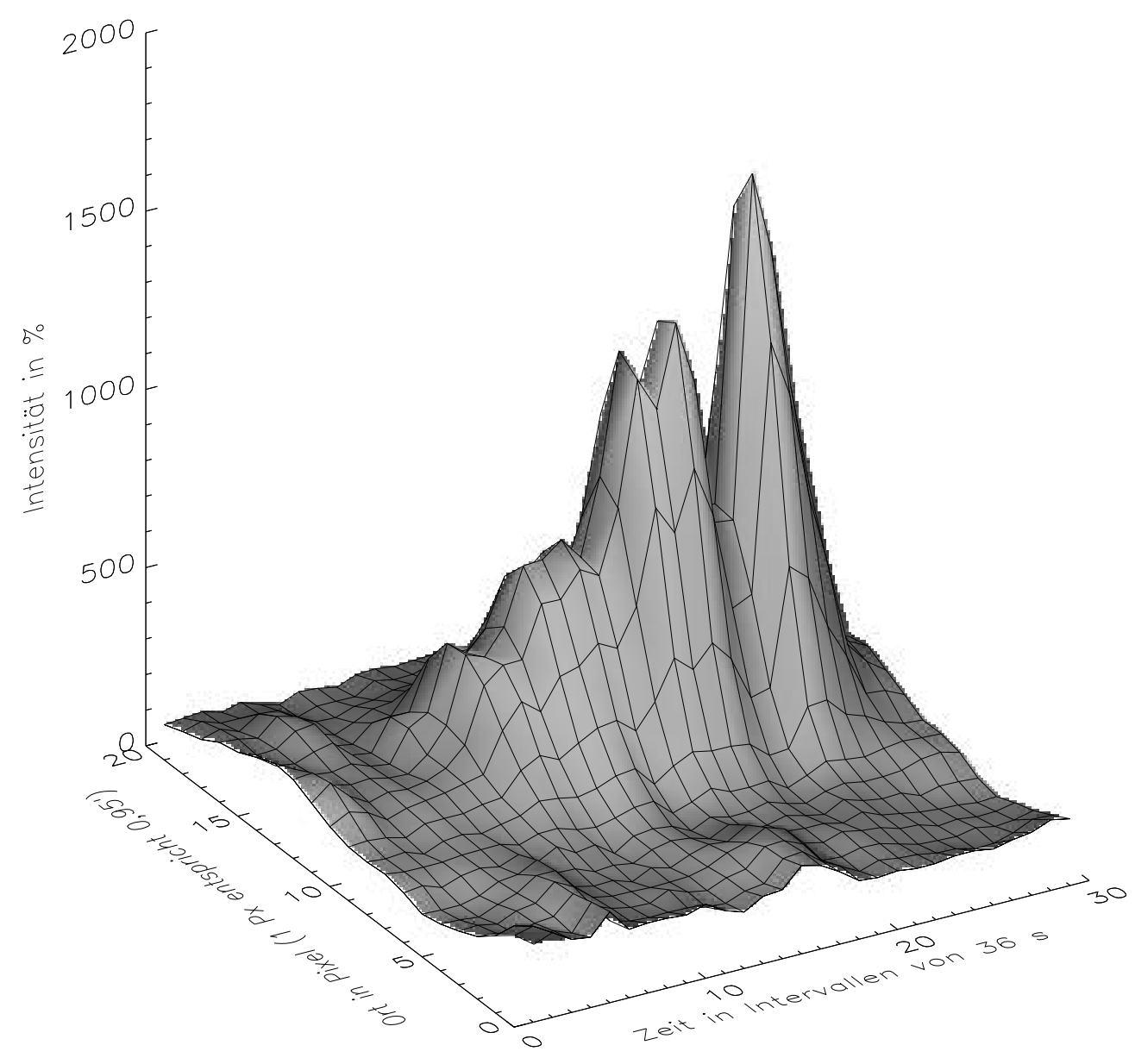

Abbildung 10.14: Dargestellt ist der $1080 \mathrm{~s}(18 \mathrm{~min}) \times 19^{\prime \prime}$ große Bereich maximaler Aufhellung aus dem $x t$-Diagramm der C IV-Linie. Auf der $z$-Achse ist die Intensität in \% der mittleren Intensität aufgetragen. 


\section{Analyse der SUMER-Spektren}

In Abb. 11.1 sind $3 x t$-Diagramme dargestellt, die jeweils den ganzen Zeitbereich der SUMER-Beobachtungen überstreichen (8:16:49 UT bis 11:05:55 UT). Von oben nach unten sind dies die Magnetfelder (sie reißen um 9:54:13 UT ab), die C IV- und Ne VIIIIntensitäten (vgl. auch Abb. 10.2, 10.6, 10.7). In den Diagrammen sind 9 verschiedene Bereiche durch weiße Kästchen hervorgehoben. Im folgenden sollen diese detailliert untersucht werden. Für die Untersuchung hilfreich ist der Zeitstrahl Abb 11.2. Hier wurden über den Zeitraum gemeinsamer Beobachtung $\Delta t=5844 \mathrm{~s}$ zum einen die Intervallzeiten der 44 GCT-Magnetogramme (Nr. 3 bis 46) und zum anderen die der 164 SUMER-Spektren graphisch dargestellt. Die Abbildung ermöglicht eine schnelle Zuordnung zwischen einzelnen SUMER-Spektren und Magnetogrammen (Abb. 8.1 bis 8.2 bzw. 8.6 bis 8.9) bzw. Potentialfeldextrapolationen (Abb. 9.8 bis 9.15).

\subsection{Bereich 1}

In Abb. 11.3 ist der erste Bereich auf dem Hintergrund des C IV-Diagramms markiert. Dieser Bereich überstreicht $792 \mathrm{~s}$ (13 min $12 \mathrm{~s}$ ) und hat eine räumliche Ausdehnung von 9," 5. Er weist sowohl im Lichte der C IV- als auch im Lichte der Ne VIII-Linie eine sich nur geringfügig wandelnde Struktur schwacher Aufhellung auf (vgl. Abb 11.1). Das Magnetogramm zeigt in diesem Bereich schwachen bis mäßigen Fluß von max. $\pm 6 \cdot 10^{8} \mathrm{~Wb}$ gemischter Polarität. Die Abb. 11.4 und 11.5 zeigen die 22 C IV- bzw. Ne VIII-Spektren (Nr. 24 bis 45) aus diesem Bereich in Negativdarstellung. Die zeitliche Reihenfolge geht von links nach rechts und von oben nach unten. Die kleinen vertikalen Striche oben und unten in jedem Spektrum markieren die Position der Ruhewellelänge $\lambda_{0}$. Die jeweils zwei horizontalen Striche links und rechts in jedem Spektrum markieren den betrachteten Ortsbereich. Jedem Spektrum stehen in Dispersionsrichtung, einem Intervall von $\Delta \lambda \approx 0,21 \mathrm{~nm}, 50$ Pixel zur Verfügung; gleiches gilt auch für die Ne VIII-Spektren. Für die C IV-Spektren ergibt sich aus einer Dispersion von 4, $1866 \frac{\mathrm{pm}}{\text { Pixel }}$ bei einem $\lambda_{0}$ von 154,821 nm mittels Doppler-Effekt rechnerisch ein Verhältnis von $8,107 \frac{\mathrm{km} / \mathrm{s}}{\text { Pixel }}$. Für die Spektren der Ne VIII-Linie 8, $166 \frac{\mathrm{km} / \mathrm{s}}{\text { Pixel }}$ bei einer Dispersion von 4, $1971 \frac{\mathrm{pm}}{\text { Pixel }}$ und einem $\lambda_{0}$ von 154,0846 nm (wegen Beobachung in zweiter Ordnung). Läßt man für die Position der Ruhewellenlänge (für C IV Pixel-Nr. 32, für Ne VIII Pixel-Nr. 29) einen Pixel unberücksichtigt, so erhält man einen maximal darstellbaren Geschwindigkeitsbe- 


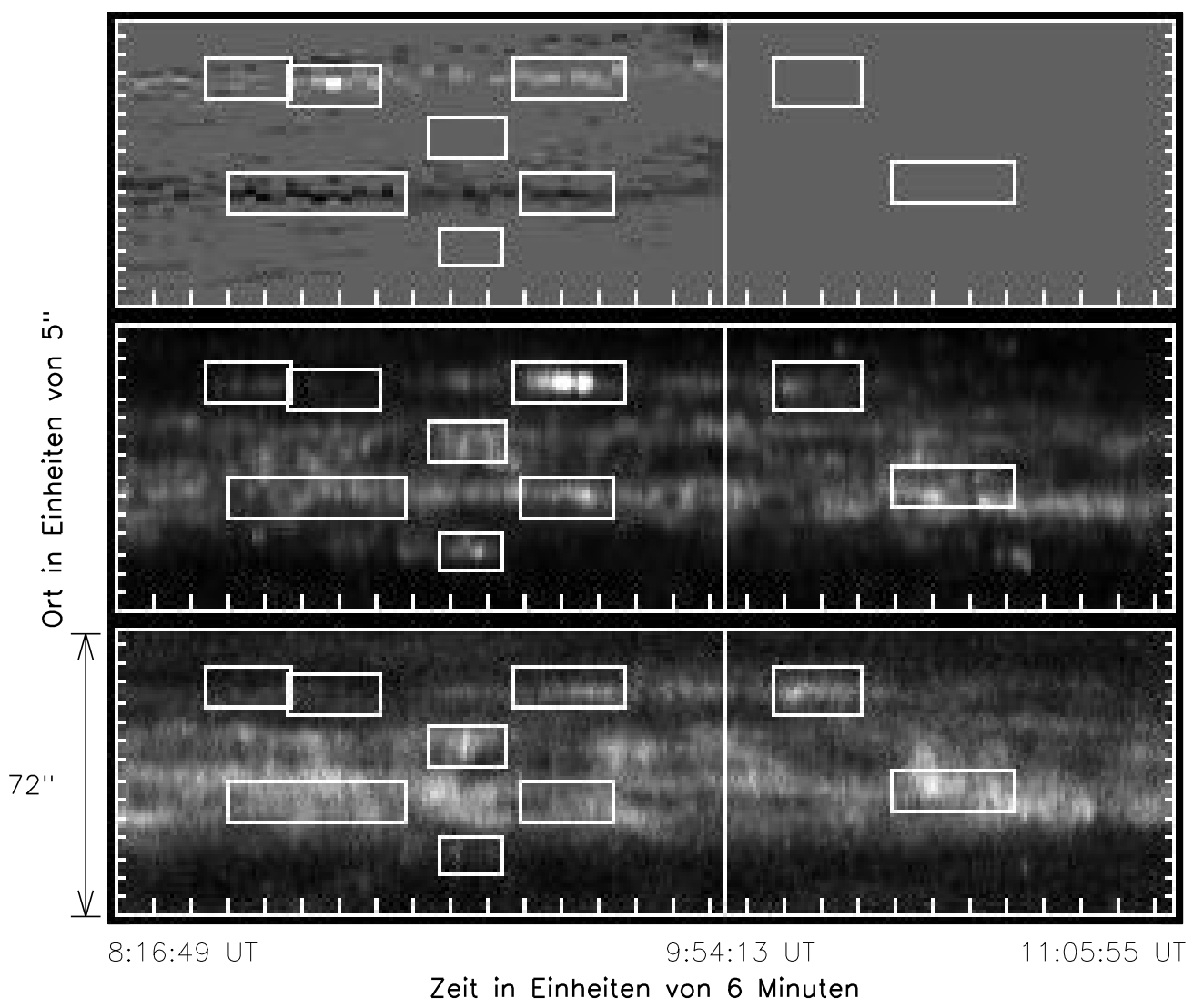

Abbildung 11.1: Es sind $3 x$-Diagramme dargestellt, die jeweils den ganzen Zeitbereich der SUMER-Beobachtungen überstreichen (8:16:49 UT bis 11:05:55 UT). Von oben nach unten sind dies das Magnetogramm (es reißt um 9:54:13 UT ab), das C IV- und das Ne viII-Filtergramm (vgl. auch Abb. 10.2, 10.6, 10.7). In den Diagrammen sind 9 verschiedene Bereiche durch weiße Kästchen hervorgehoben, die im folgenden detailliert untersucht werden. 


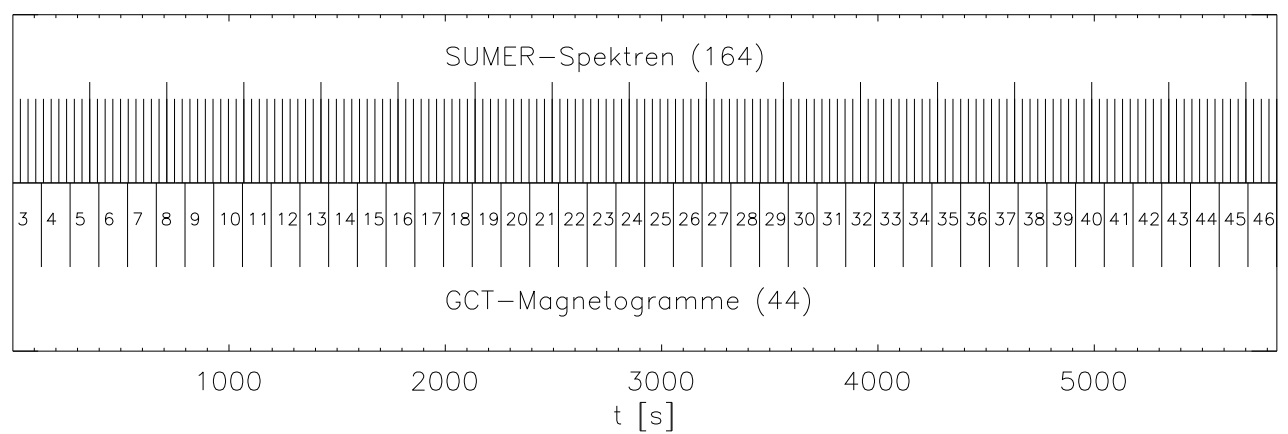

Abbildung 11.2: Zeitstrahl für eine schnelle Zuordnung zwischen einzelnen SUMERSpektren und Magnetogrammen (Abb. 8.1 bis 8.2 bzw. 8.6 bis 8.9 ) bzw. Potentialfeldextrapolationen (Abb. 9.8 bis 9.15). Es wurden über den Zeitraum gemeinsamer Beobachtung $\Delta t=5844 \mathrm{~s}$ zum einen die Intervallzeiten der 44 GCT-Magnetogramme (Nr. 3 bis 46) und zum anderen die der 164 SUMER-Spektren graphisch dargestellt.

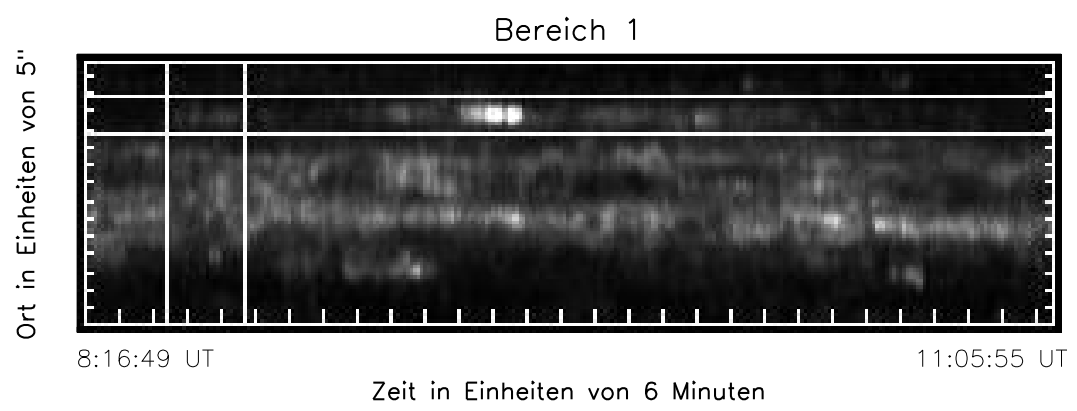

Abbildung 11.3: Bereich Nr. 1 auf dem Hintergrund des C IV-Diagramms. 


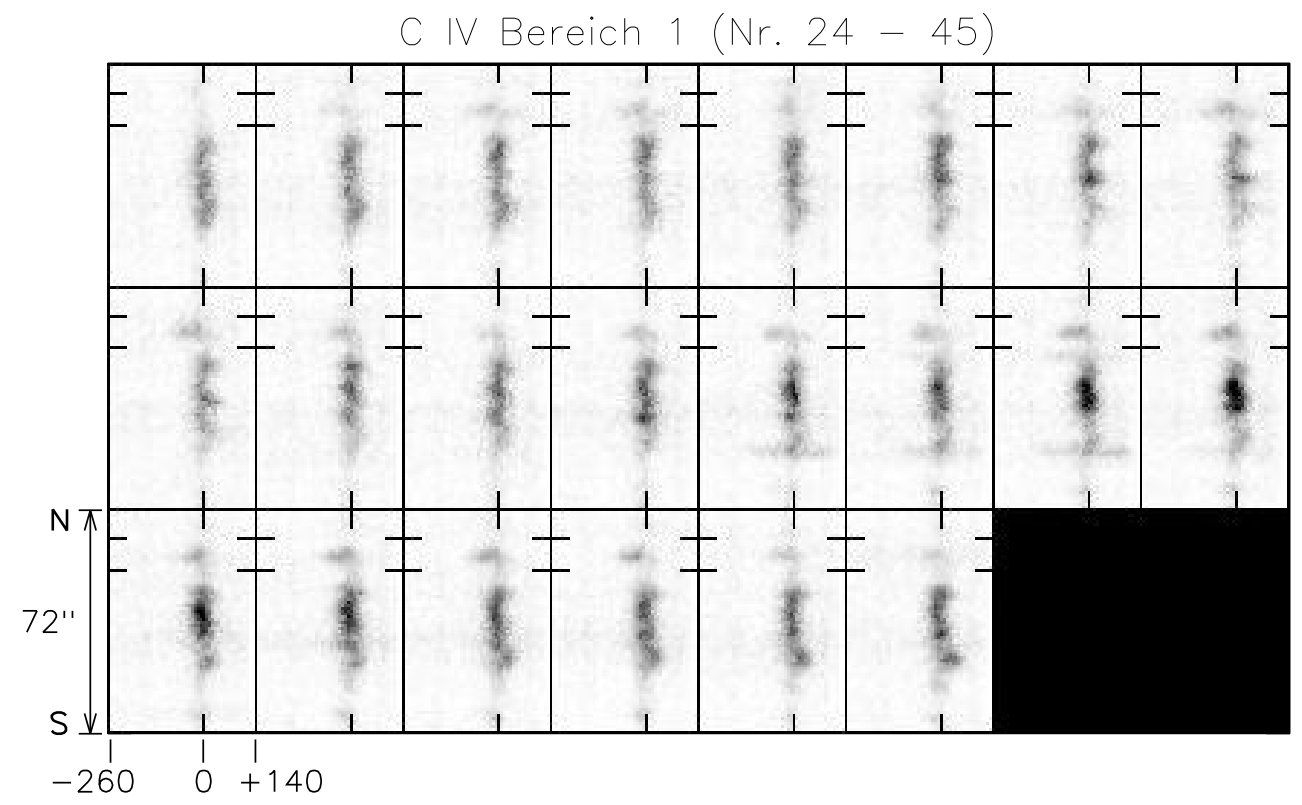

Geschwindigkeit in $\mathrm{km} / \mathrm{s}$

Abbildung 11.4: Spektren der CIV-Linie aus dem Bereich $1(\Delta \lambda \approx 0,21 \mathrm{~nm}, \Delta x \approx$ $\left.72^{\prime \prime}\right)$. Die kleinen vertikalen Striche oben und unten in jedem Spektrum markieren die $\lambda_{0}$-Position. Die jeweils zwei horizontalen Striche links und rechts in jedem Spektrum markieren den betrachteten Ortsbereich. Der zeitliche Abstand zwischen den Spektren beträgt $36 \mathrm{~s}$. 


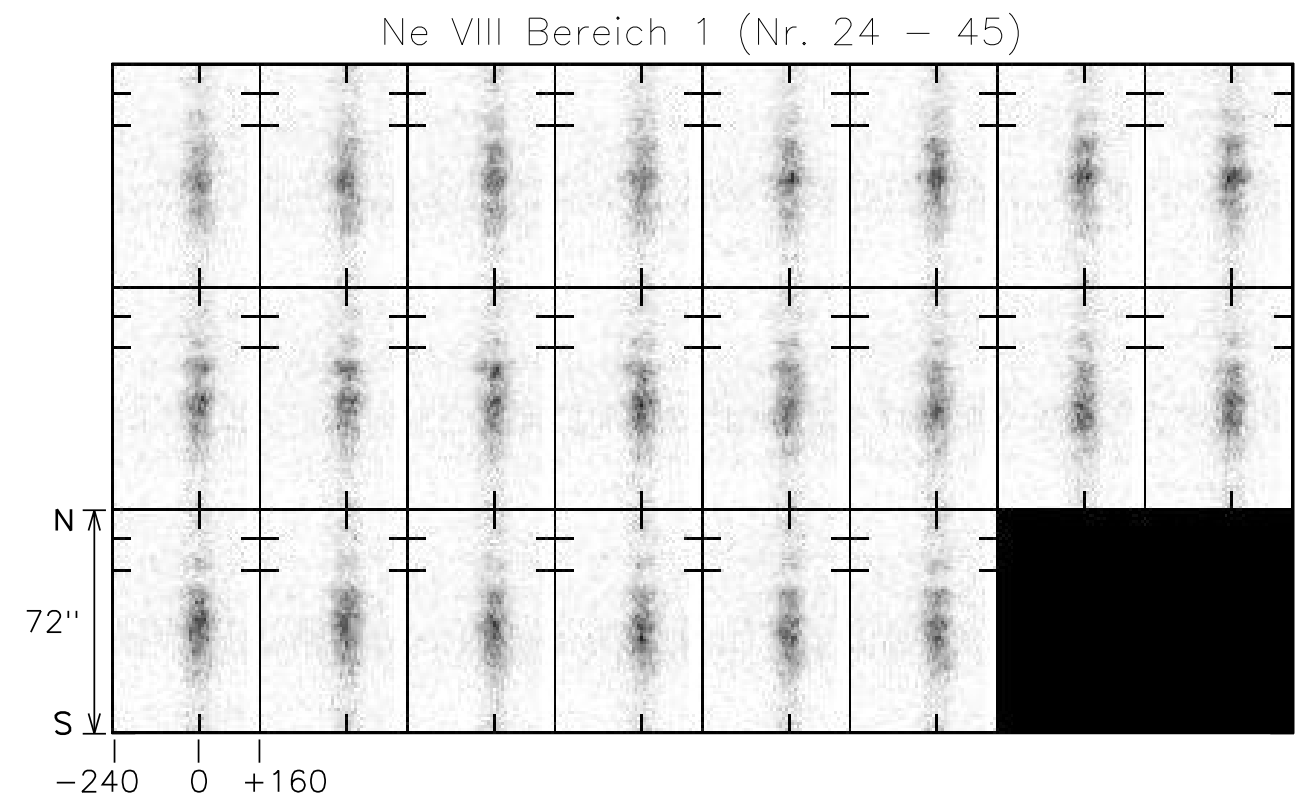

Geschwindigkeit in $\mathrm{km} / \mathrm{s}$

Abbildung 11.5: Spektren der Ne vin-Linie aus dem Bereich $1(\Delta \lambda \approx 0,21 \mathrm{~nm}, \Delta x \approx$ $\left.72^{\prime \prime}\right)$. Die kleinen vertikalen Striche oben und unten in jedem Spektrum markieren die $\lambda_{0}$-Position. Die jeweils zwei horizontalen Striche links und rechts in jedem Spektrum markieren den betrachteten Ortsbereich. Der zeitliche Abstand zwischen den Spektren beträgt $36 \mathrm{~s}$. 


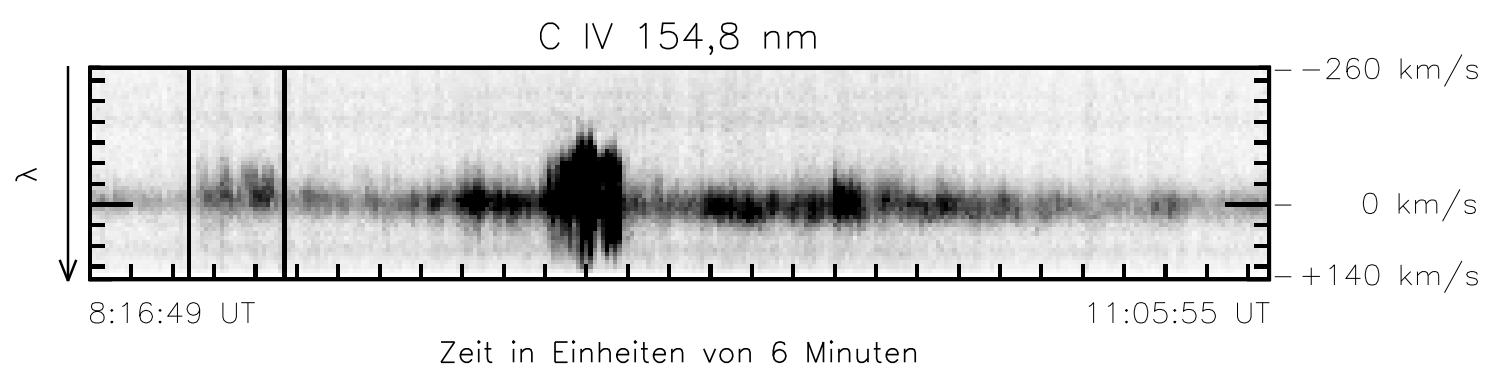

Abbildung 11.6: $\lambda t$-Diagramm C IV-Linie aus dem Bereich 1. Die zwei schwarzen vertikalen Linien grenzen den betrachteten Bereich zeitlich ein. Die Einheit auf der Geschwindigkeitsachse beträgt $\sim 40 \mathrm{~km} / \mathrm{s}$.

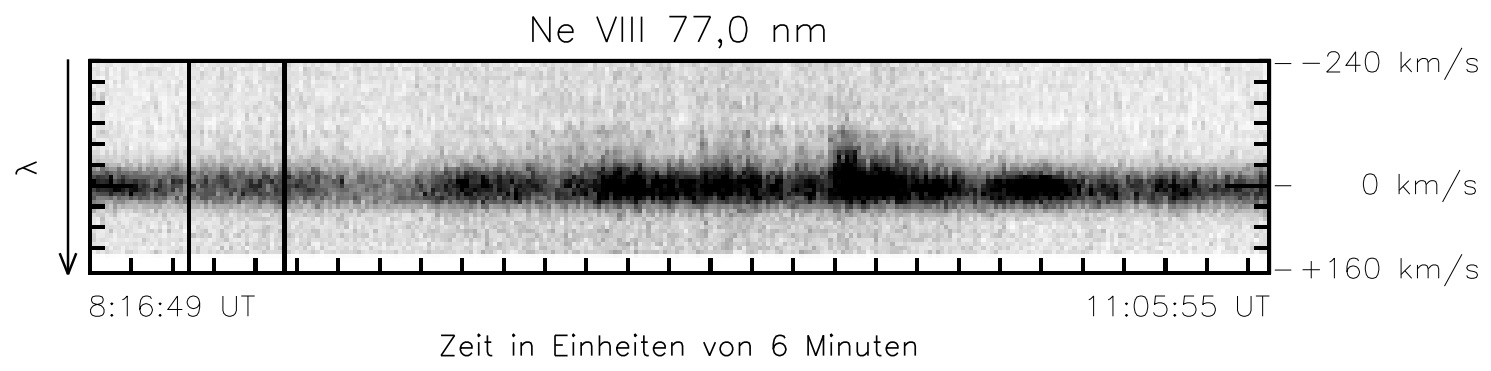

Abbildung 11.7: $\lambda t$-Diagramm Ne VIII-Linie aus dem Bereich 1. Die zwei schwarzen vertikalen Linien grenzen den betrachteten Bereich zeitlich ein. Die Einheit auf der Geschwindigkeitsachse beträgt $\sim 40 \mathrm{~km} / \mathrm{s}$.

reich von $\sim-260 \mathrm{~km} / \mathrm{s}$ (blau-verschoben) bis $\sim+140 \mathrm{~km} / \mathrm{s}$ (rot-verschoben) für die C IV-Spektren und $\sim-240 \mathrm{~km} / \mathrm{s}$ bis $\sim+160 \mathrm{~km} / \mathrm{s}$ für die Ne VIII-Spektren. Die Abbildungen 11.6 und 11.7 zeigen für C IV bzw. Ne VIII sogenannte $\lambda t$-Diagramme. In diesen Diagrammen wurden die in den Spektren der Abb. 11.4 und 11.5 markierten Ortsbereiche gemittelt und gegen die Zeit aufgetragen. Die zwei schwarzen vertikalen Linien markieren den betrachteten Zeitbereich. In den Abb. 11.8 und 11.9 sind die gemittelten Spektrumsausschnitte, die für die $\lambda t$-Diagramme angefertigt wurden, nochmals einzeln dargestellt. Die vertikale gepunktete Linie markiert die $\lambda_{0}$-Position und das gestrichelte Linienprofil ist das über alle Spektren eines Beobachtungstages gemittelte Bezugsprofil einer Linie.

Folgt man mit Hilfe der Abb. 11.4, 11.6, 11.8 der Entwicklung der C IV-Linie im ausgewählten Bereich, so findet man, ausgehend von dem Spektrumsausschnitt Nr. 24 in Abb. 11.8, wo die gemessenen Werte deutlich unter dem mittleren Profil liegen, eine immer wieder ansteigende und abfallende Blauverschiebung mit relativen Maxima bei Nr.27, 32, 39 und 41 bis 43 . Hierbei werden Geschwindigkeiten von bis zu $\sim-55 \mathrm{~km} / \mathrm{s}$ erreicht. Die Ne vinI-Linie (vgl. Abb. 11.5, 11.7, 11.9) bleibt hingegen fast völlig in Ruhe. 

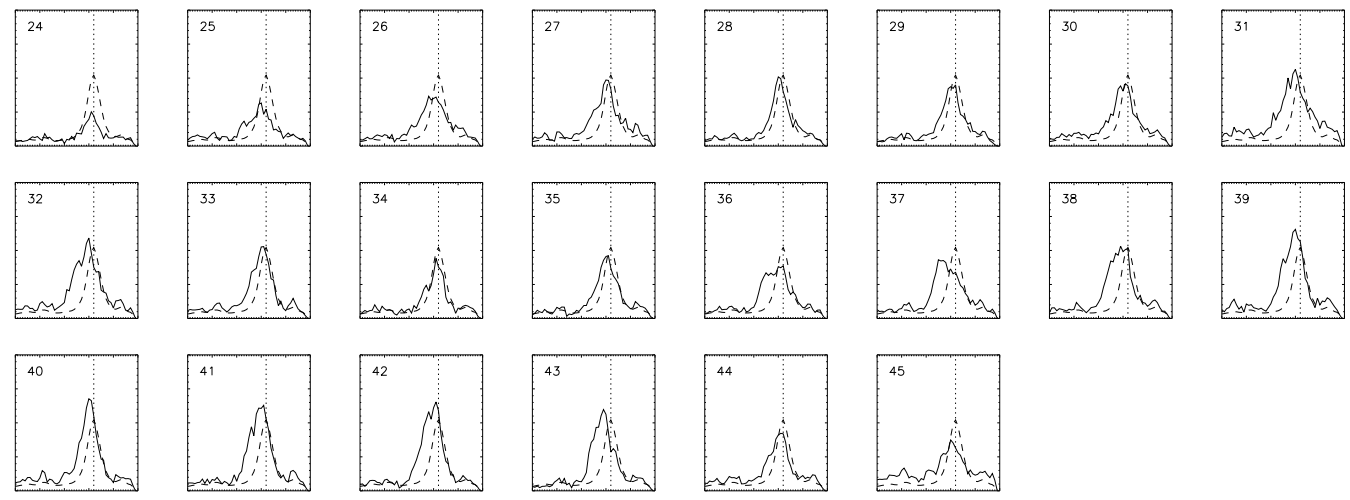

Abbildung 11.8: Über den betrachteten Ortsbereich gemittelte C IV-Spektren aus dem betrachteten Zeitraum. Die Intensität ist in relativen Einheiten gegen die Wellenlänge aufgetragen ( $\lambda$ steigt nach rechts an). Die vertikale, gepunktete Linie markiert die $\lambda_{0}$-Position, und das gestrichelte Profil gibt den gemittelten Spektrumsausschnitt wieder.
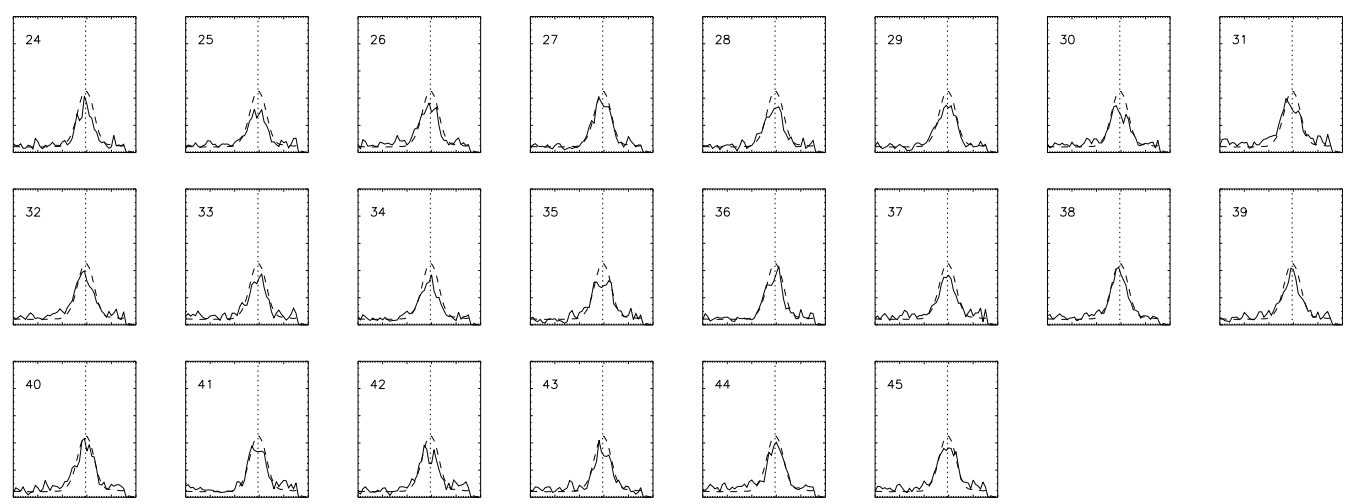

Abbildung 11.9: Über den betrachteten Ortsbereich gemittelte Ne VIII-Spektren aus dem betrachteten Zeitraum. Die Intensität ist in relativen Einheiten gegen die Wellenlänge aufgetragen ( $\lambda$ steigt nach rechts an). Die vertikale, gepunktete Linie markiert die $\lambda_{0}$-Position, und das gestrichelte Profil gibt den gemittelten Spektrumsausschnitt wieder. 


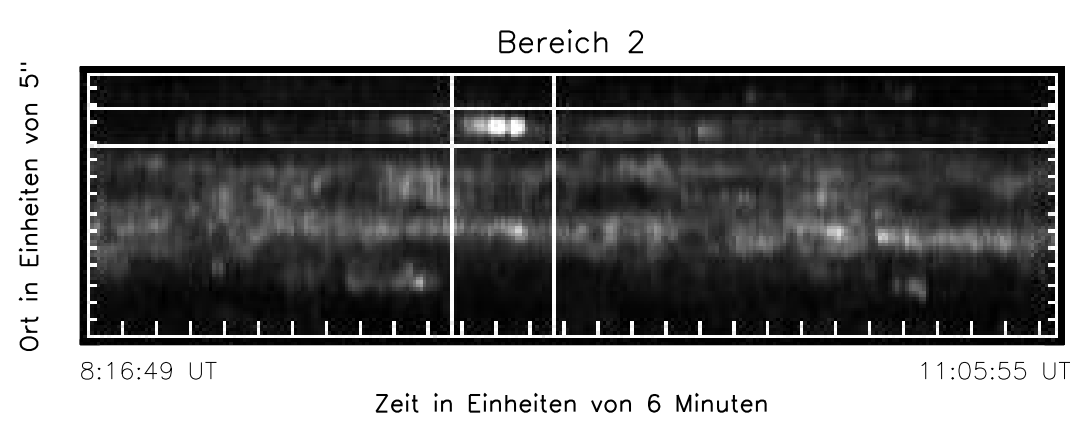

Abbildung 11.10: Bereich Nr. 2 auf dem Hintergrund des C IV-Diagramms.

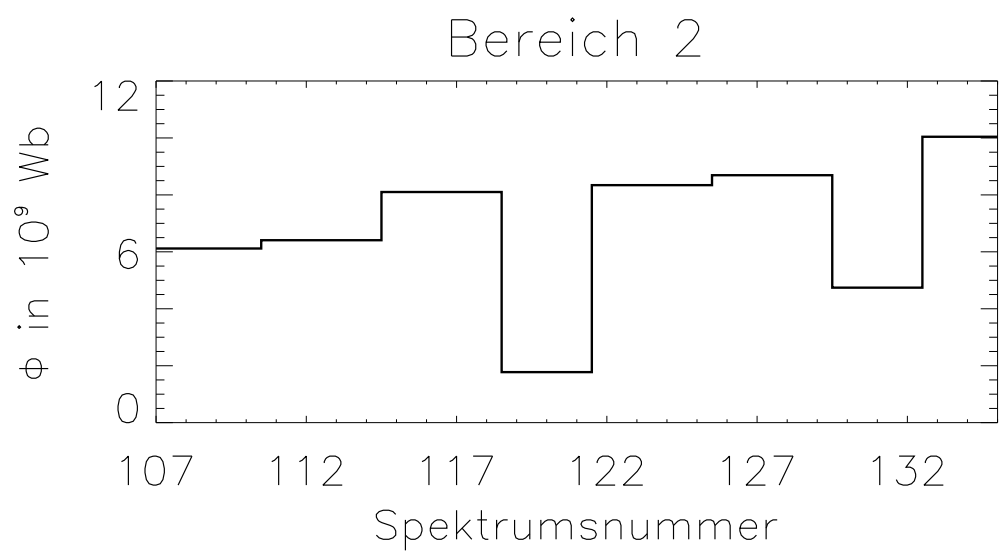

Abbildung 11.11: Über den betrachteten Ortsbereich integrierter magnetischer Fluß.

\subsection{Bereich 2}

In Abb. 11.10 ist der Bereich 2 (vgl. auch Abb. 11.1) auf dem Hintergrund des CIV$x$-Diagramms markiert. Er hat eine räumliche Ausdehnung von 9," 5 und eine zeitliche von $1044 \mathrm{~s}$ (17 min 24s). Dieser Bereich beinhaltet das absolute Helligkeitsmaximum der C IV-Aufzeichnung des ganzen Tages (sogar der ganzen Beobachtungskampagne vom 4.5 bis 10.5.1999), weist aber in der Ne VIII-Linie nur eine durchschnittliche bis schwache Aufhellung auf. Der Abb. 11.1 kann man entnehmen, daß dieser Bereich in einem Gebiet mit ausschließlich positiver Polarität liegt. Der magnetische Fluß in der Mitte des betrachteten Bereichs beginnt bei etwa $6 \cdot 10^{8} \mathrm{~Wb}$, steigt nach ca. 4,5 Minuten für $\sim 2$ Minuten auf $1,4 \cdot 10^{9} \mathrm{~Wb}$, fällt sodann für etwa zwei Minuten auf einen Wert kleiner $3 \cdot 10^{8} \mathrm{~Wb}$ um anschließend wieder für ca. 4,5 Minuten auf knapp $1 \cdot 10^{9} \mathrm{~Wb}$ anzusteigen. Danach fällt der magnetische Fluß wieder für etwa zwei Minuten auf einen Wert um $3 \cdot 10^{8} \mathrm{~Wb}$ und steigt für die verbleibende Zeit wieder auf einen Wert von ca. $1,4 \cdot 10^{9} \mathrm{~Wb}$, allerdings ca. $3^{\prime \prime}$ nördlich. Dieser Verlauf wird qualitativ auch durch 


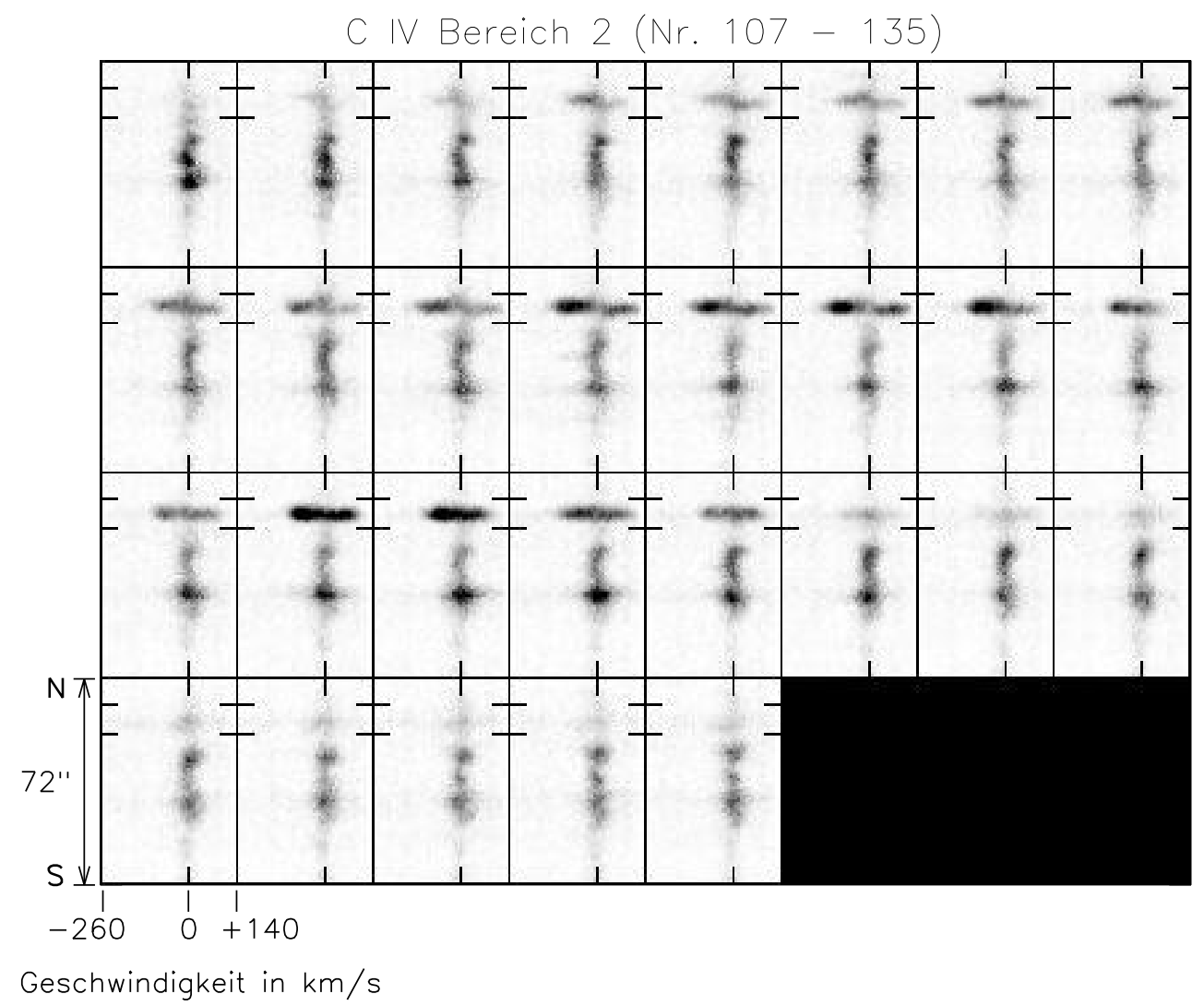

Abbildung 11.12: Spektren der C IV-Linie aus dem Bereich $2(\Delta \lambda \approx 0,21 \mathrm{~nm}, \Delta x \approx$ $\left.72^{\prime \prime}\right)$. Die kleinen vertikalen Striche oben und unten in jedem Spektrum markieren die $\lambda_{0}$-Position. Die jeweils zwei horizontalen Striche links und rechts in jedem Spektrum markieren den betrachteten Ortsbereich. Der zeitliche Abstand zwischen den Spektren beträgt $36 \mathrm{~s}$. 


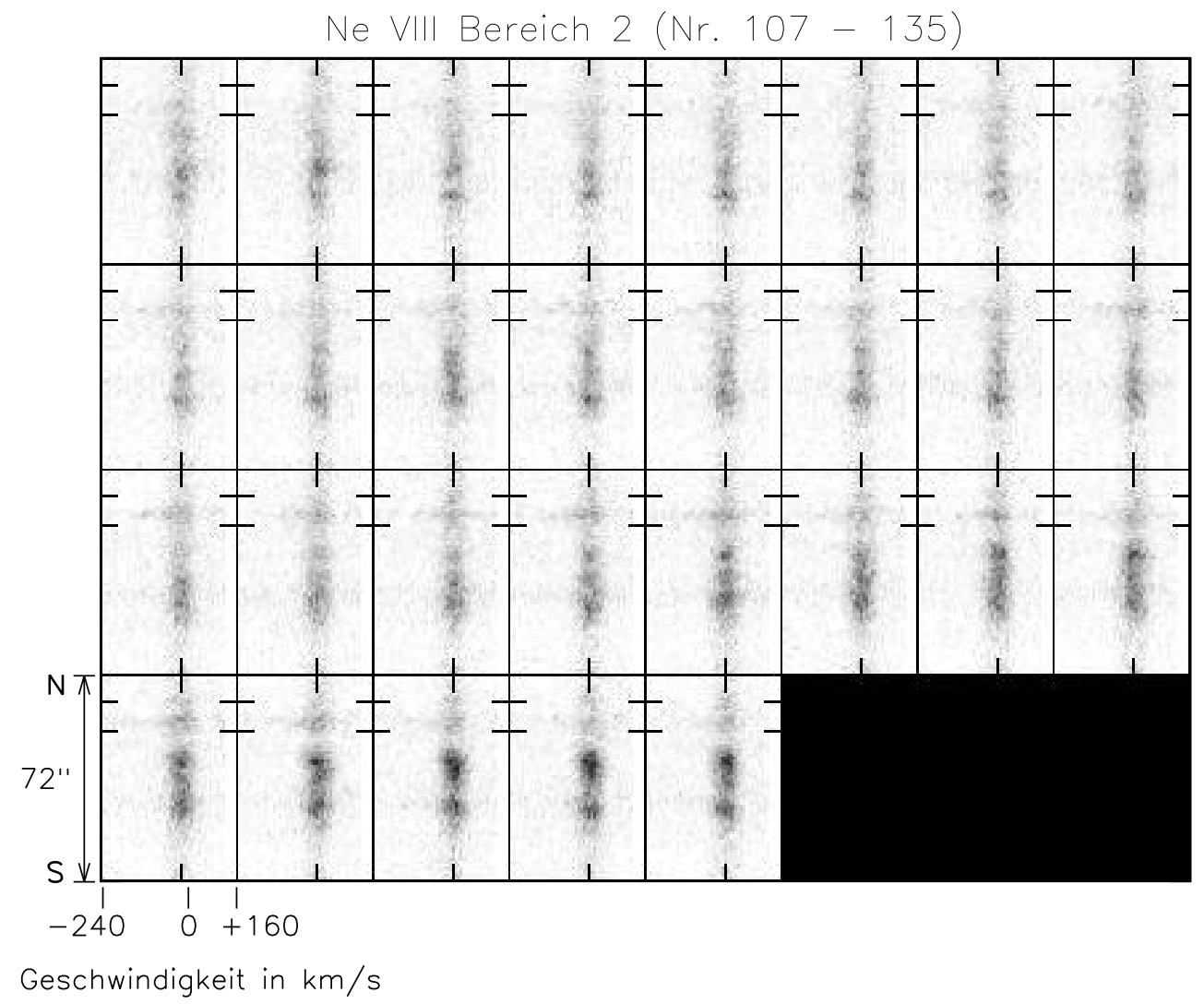

Abbildung 11.13: Spektren der Ne VIII-Linie aus dem Bereich $2(\Delta \lambda \approx 0,21 \mathrm{~nm}, \Delta x \approx$ $\left.72^{\prime \prime}\right)$. Die kleinen vertikalen Striche oben und unten in jedem Spektrum markieren die $\lambda_{0}$-Position. Die jeweils zwei horizontalen Striche links und rechts in jedem Spektrum markieren den betrachteten Ortsbereich. Der zeitliche Abstand zwischen den Spektren beträgt 36 s.

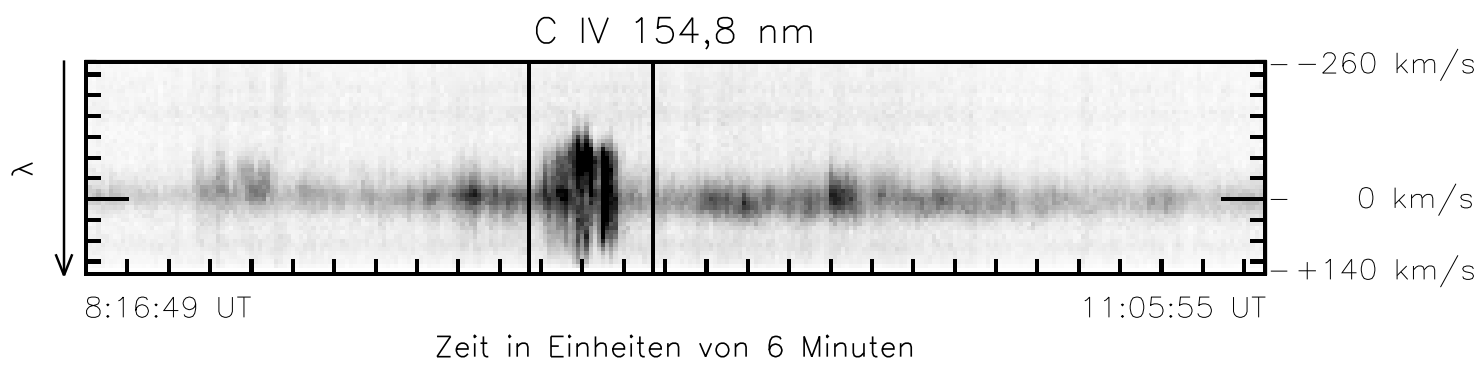

Abbildung 11.14: $\lambda t$-Diagramm C IV-Linie aus dem Bereich 2. Die zwei schwarzen vertikalen Linien grenzen den betrachteten Bereich zeitlich ein. Die Einheit auf der Geschwindigkeitsachse beträgt $\sim 40 \mathrm{~km} / \mathrm{s}$. 


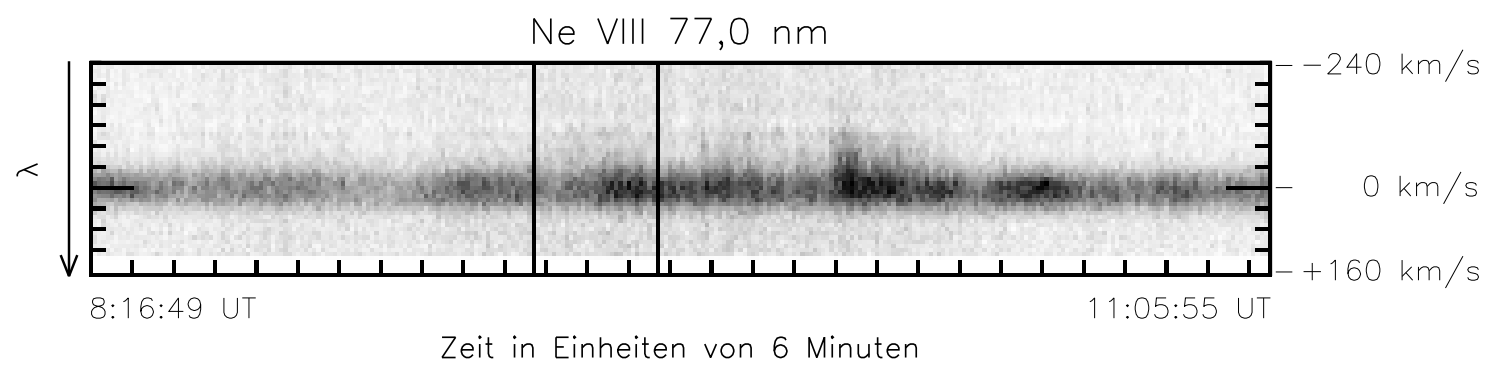

Abbildung 11.15: $\lambda t$-Diagramm Ne vinI-Linie aus dem Bereich 2. Die zwei schwarzen vertikalen Linien grenzen den betrachteten Bereich zeitlich ein. Die Einheit auf der Geschwindigkeitsachse beträgt $\sim 40 \mathrm{~km} / \mathrm{s}$.
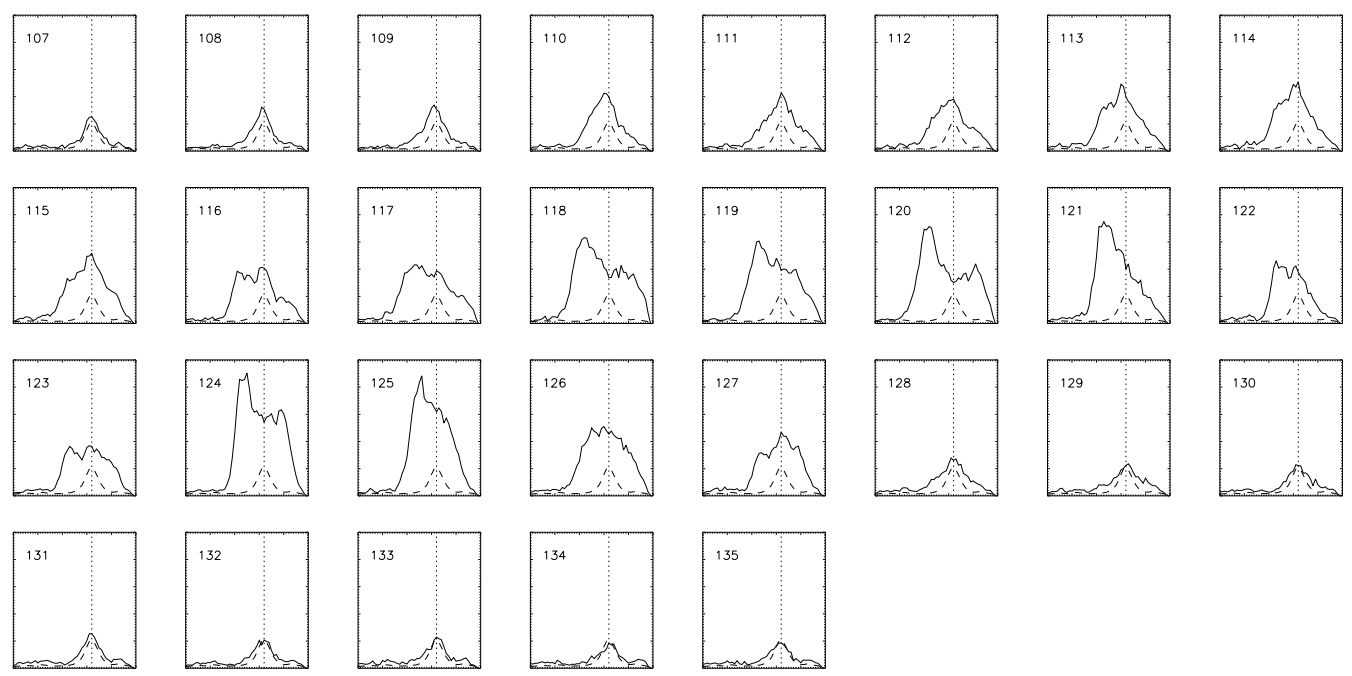

Abbildung 11.16: Über den betrachteten Ortsbereich gemittelte C IV-Spektren aus dem betrachteten Zeitraum. Die Intensität ist in relativen Einheiten gegen die Wellenlänge aufgetragen ( $\lambda$ steigt nach rechts an). Die vertikale, gepunktete Linie markiert die $\lambda_{0}$-Position, und das gestrichelte Profil gibt den gemittelten Spektrumsausschnitt wieder. 

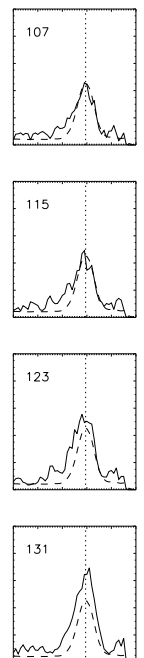
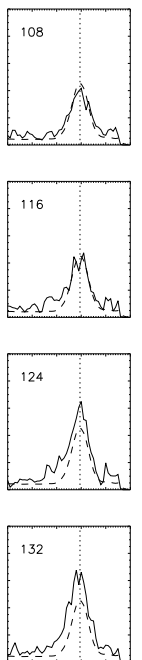
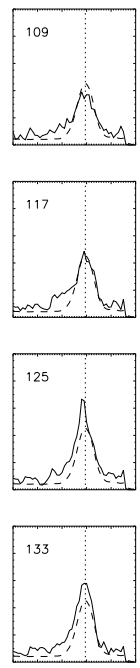
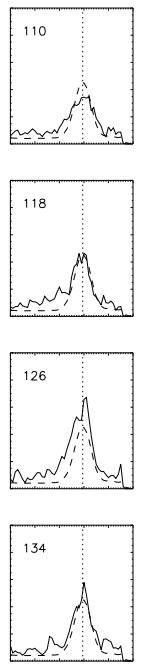
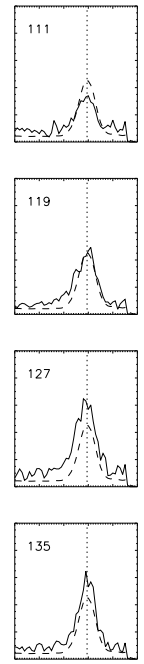
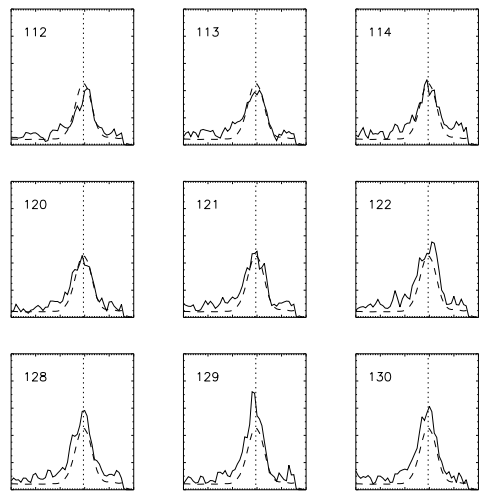

Abbildung 11.17: Über den betrachteten Ortsbereich gemittelte Ne viII-Spektren aus dem betrachteten Zeitraum. Die Intensität ist in relativen Einheiten gegen die Wellenlänge aufgetragen ( $\lambda$ steigt nach rechts an). Die vertikale, gepunktete Linie markiert die $\lambda_{0}$-Position, und das gestrichelte Profil gibt den gemittelten Spektrumsausschnitt wieder.

den in Abb. 11.11 dargestellten Graphen des über den Ortsbereich des Bereichs 2 integrierten magnetischen Flusses wiedergegeben. Im Zusammenhang mit diesem Graphen sollte man sich folgendes vergegenwärtigen: Die eingetragenen Werte sind die über das Gebiet von $1^{\prime \prime} \times 9,{ }^{\prime \prime} 5\left(\widehat{\approx} 5000000 \mathrm{~km}^{2}\right)$ integrierten magnetischen Flüsse. Die maximale im betrachteten Zeitraum auftretende Differenz dieses integrierten magnetischen Flusses beträgt grob $\Delta \phi=8 \cdot 10^{9} \mathrm{~Wb}$. Schwankungen im magnetischen Fluß können sich durch von außen in die betrachtete Fläche hinzukommende oder sich aus der Fläche herausbewegende Bereiche magnetischen Flusses einer Polarität ergeben. Weiterhin besteht die Möglichkeit von zusätzlichem aus dem Sonnenkörper heraustretenden magnetischen Flusses, der, je nach Polarität, Abschwächung oder Verstärkung bedeuten kann. In dem nicht aufgelösten Flächenelement können eine Vielzahl von Konfigurationen der magnetischen Struktur zum selben beobachteten Ergebnis führen. Ferner können Variationen der Sichtbedingungen (engl. Seeing) während der Beobachtung z.B. zu Bildversetzungen führen, die sich dann auch als Schwankungen des beobachteten magnetischen Flusses niederschlagen können. Darüber hinaus erklärt sich der "eckige" Verlauf des Graphen über mehrere SUMER-Spektren aus der unterschiedlichen Zeitauflösung von GCT- und SUMER-Beobachtungen. Es mußten die $x t$-Magnetogramme, die aus diskreten Messungen mit zeitlichen Abständen von etwas mehr als 2 Minuten zusammengesetzt wurden durch Vergrößerung auf einen mit den SUMER-Daten vergleichbaren Maßstab gebracht werden. Die Abbildungen 11.12, 11.14, 11.16 für die C IV-Linie und Abbildungen 11.13, 


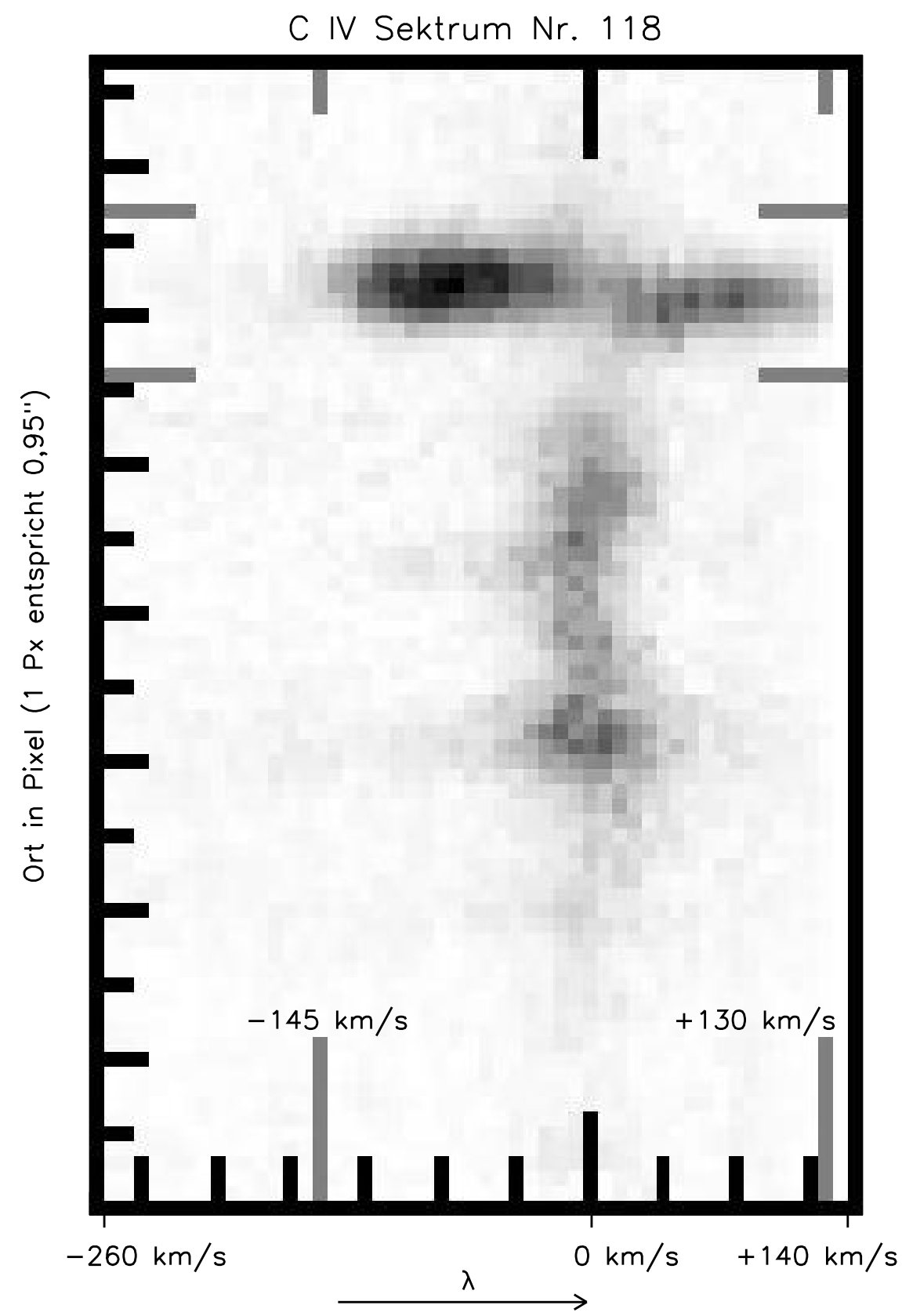

Pixel (1 Px entspricht 4,1866 pm bzw. $8 \mathrm{~km} / \mathrm{s}$ )

Abbildung 11.18: C IV-Spektrum Nr. $118\left(\Delta \lambda \approx 0,21 \mathrm{~nm}, \Delta x=76\right.$ Pixel $\cdot 0,95 \frac{\prime \prime}{\text { Pixel }} \approx$ $\left.72^{\prime \prime}\right)$. Auf der Ortsachse wurde alle 5 Pixel eine Markierung gesetzt. Die Einheit auf der Geschwindigkeitsachse beträgt $\sim 40 \mathrm{~km} / \mathrm{s}$. Die kleinen vertikalen Striche oben und unten in jedem Spektrum markieren die $\lambda_{0}$-Position. Die jeweils zwei horizontalen Striche links und rechts in jedem Spektrum markieren den betrachteten Ortsbereich. 

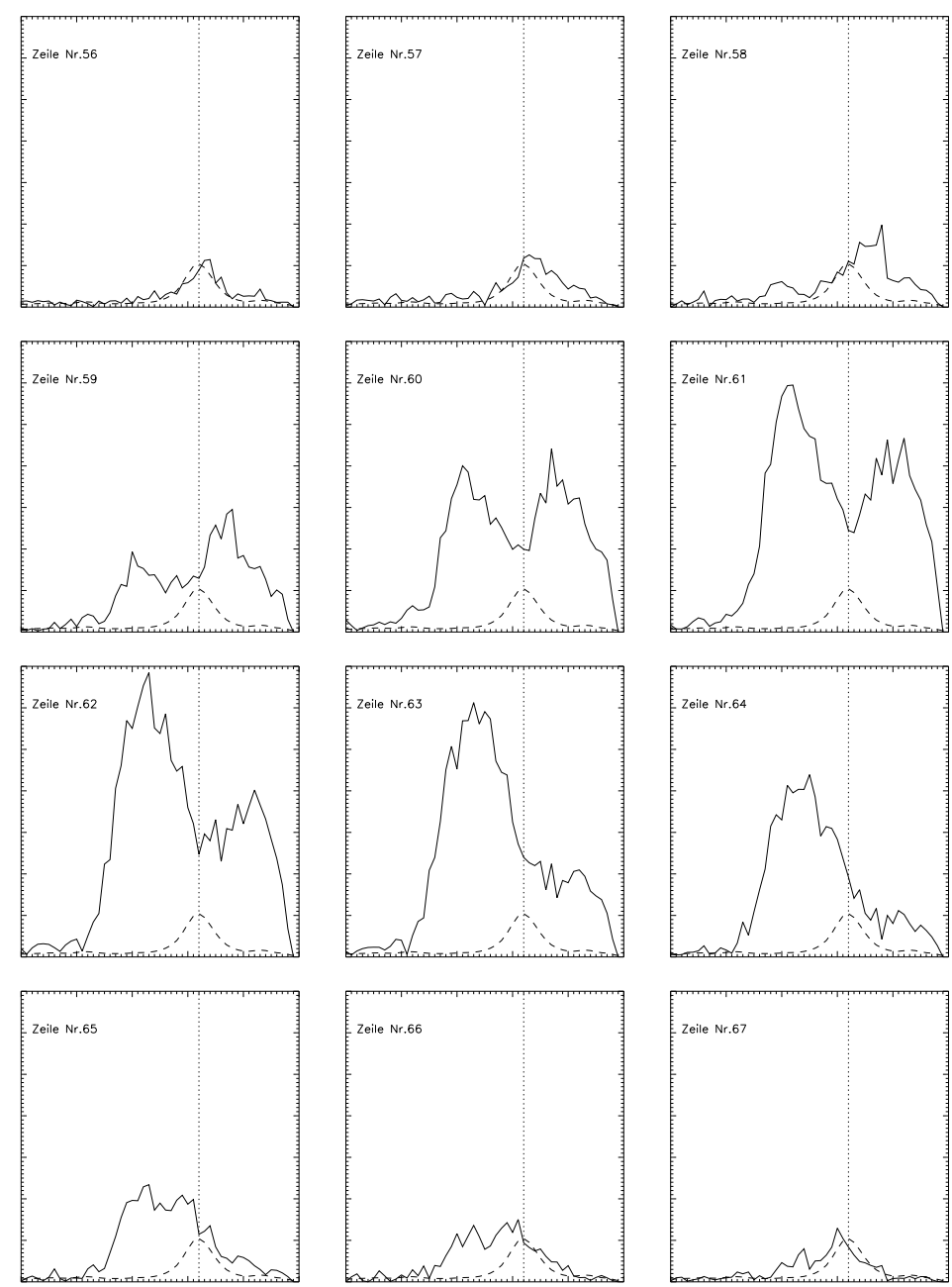

Abbildung 11.19: Aus dem markierten Bereich der Abb. 10.16 zeilenweise entnommene Spektrumsausschnitte. Die Intensität ist in relativen Einheiten gegen die Wellenlänge aufgetragen ( $\lambda$ steigt nach rechts an). Die vertikale, gepunktete Linie markiert die $\lambda_{0}$-Position, und das gestrichelte Profil gibt den gemittelten Spektrumsausschnitt wieder. 
11.15, 11.17 für die Ne VIII-Linie wurden in gleicher Weise angefertigt, wie die schon beschriebenen Abbildungen 11.4, 11.6, 11.8 bzw. 11.5, 11.7, 11.9. Im Verlauf des betrachteten Zeitraums findet in der C IV-Linie ein Ausbruch (engl. burst) von starken explosiven Ereignissen statt, der etwa 12 Minuten dauert, siehe $\lambda t$-Diagramm Abb. 11.14. Es ist festzustellen, daß sich der Ausbruch als ganzes deutlich flacher aufbaut, als er abfällt. Zeitlich dagegen verschoben zeigt die Ne VIII-Linie nur marginale Blauverschiebung, siehe Abb. 11.13, 11.15, 11.17. Ausgehend von dem gemittelten Profil Abb. 11.16 Nr. 107 (bzw. dem Spektrum Abb. 11.12 Nr. 107), wo die beobachtete Intensität mit der mittleren Intensität des ganzen Tages zusammenfällt, entwickelt sich zunächst eine Blauverschiebung (Nr. 108 bis 110), die dann zu einer ansteigenden allgemeinen Verbreiterung wird (Nr. 111 bis 117), wobei der Flächenschwerpunkt (außer in Nr. 111 und 115) immer zum Blauen hin verschoben bleibt. Erstmals in Abb. 11.16 bzw. 11.12 Nr. 118 (vgl. auch Abb. 11.18, 11.19) zeigt sich eine Aufspaltung der Linie. Es werden hier Geschwindigkeiten von bis $\mathrm{zu}-145 \mathrm{~km} / \mathrm{s}$ und $+130 \mathrm{~km} / \mathrm{s}$ erreicht. Anders als im Dopplergramm Abb. 10.9, wo die Schwerpunkte der angepaßten Gaußfunktion für die Verschiebung benutzt wurden, handelt es sich hier um maximale Ausläufer der Verschiebung. Bis Nr. 120 bleibt die Aufspaltung erhalten. Bei Nr. 121 entfällt plötzlich der rotverschobene Anteil der Linie und der verbleibende Teil wird in Nr. 122 deutlich schächer. Fast wieder symmetrisch, stellt Nr. 123 ein lokales Minimum dar. Sodann wiederholt sich der Ablauf; starke Aufhellung mit Aufspaltung in zwei Komponenten (Nr. 124), Abfall der rotverschobenen Komponente (Nr. 125) und symmetrisches schwächer Werden bis zum Verschwinden (Nr. 126 bis 128). Während jedoch bei der Aufspaltung der Linie in dem Vorlauf und der Umgebung von Nr. 118 auch eine räumliche Verschiebung zwischen roter und blauer Komponente von ca. $2^{\prime \prime}$ auftrat (vgl. Abb. 11.18 und 11.19), ist in der Umgebung von Nr. 124 dies nicht der Fall. Es ist zu vermuten, daß der Ausbruch (Abb. 11.14) eine, durch die Integrationszeit von 36 s pro Spektrum verdeckte, nicht aufgelöste, Substruktur enthält. Mit der Abb. 11.2 ordnet man dem Spektrum Nr. 118 (Abb. 11.18) das Magnetogramm Nr. 34 (Abb. 8.8 unten Mitte) bzw. die Potentialfeldextrapolation Abb. 9.13 unten links zu. In dem betrachteten oberen Bereich des SUMER-Spaltes findet sich hier (vgl. Abb. 9.13 unten links) eine relativ komplizierte Feldkonfiguration, die über den gesamten Zeitraum des Ausbruchs (Spektrum Nr. 110 bis 127 entsprechend den Potentialfeldextrapolationen 32 bis 36) nachweisbar ist. 


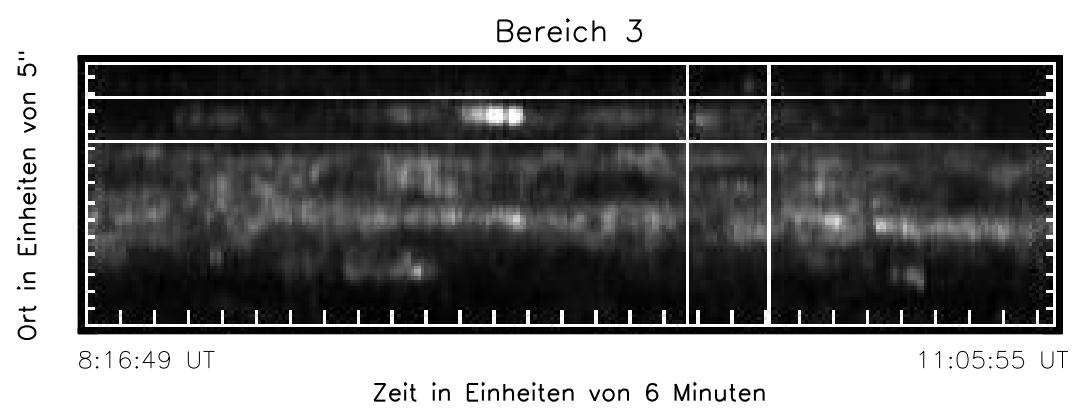

Abbildung 11.20: Bereich Nr. 3 auf dem Hintergrund des C IV-Diagramms.

\subsection{Bereich 3}

Der in Abb. 11.20 markierte Bereich 3 liegt auf der selben Höhe wie Bereich 2, ist von diesem aber 24,6 Minuten (1476s) zeitlich entfernt. Er hat eine räumliche Ausdehnung von 11", 4 und überstreicht ein Zeitintervall von 828 s (13 min 48s). Für dieses Gebiet liegen leider keine Magnetfelddaten vor (vgl. Abb. 11.1). Es ist aber insofern interessant, als daß es, anders als in den vorangegangenen Bereichen 1 und 2, mindestens zu Beginn eine, wenn auch schwache, simultane Blauverschiebung in der CIV- und Ne viII-Linie aufweist (Abb. 11.23 und 11.24). Besonders deutlich ist dies in Nr. 179 der Abb. 11.25 und $11.26 \mathrm{zu}$ sehen. Hier finden sich etwa $-80 \mathrm{~km} / \mathrm{s}$ für die C IV- und ca. $-110 \mathrm{~km} / \mathrm{s}$ für die Ne viII-Linie. Während aber in der Ne viII-Linie die Blauverschiebung mit wandelnder Stärke bis Nr. 196 anhält (also über ca. 11 Minuten), verändert sich die Verschiebung in der C IV-Linie rasch in eine allgemeine Verbreiterung, die dann schnell schwächer wird und nach etwa 4 Minuten (Nr. 186) dem mittleren Profil entspricht. 


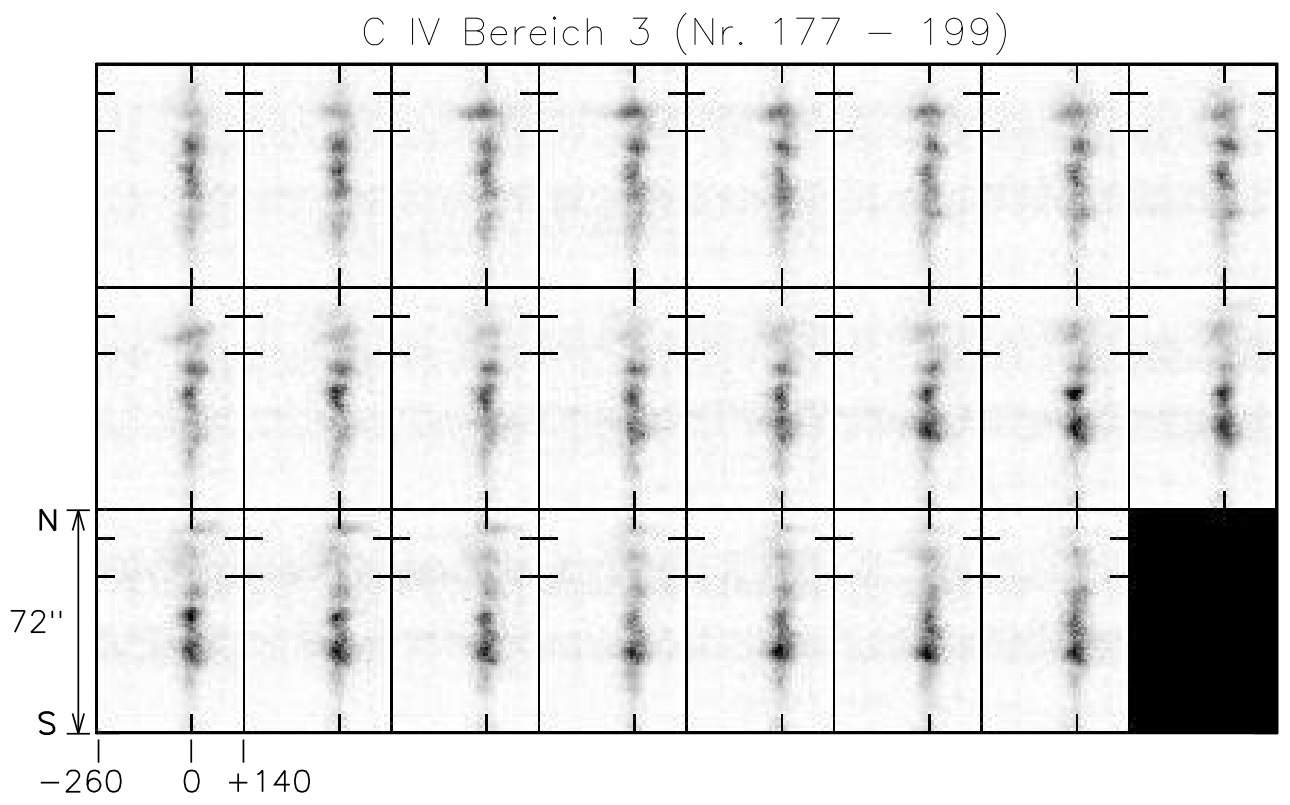

Geschwindigkeit in $\mathrm{km} / \mathrm{s}$

Abbildung 11.21: Spektren der C IV-Linie aus dem Bereich $3(\Delta \lambda \approx 0,21 \mathrm{~nm}, \Delta x \approx$ $\left.72^{\prime \prime}\right)$. Die kleinen vertikalen Striche oben und unten in jedem Spektrum markieren die $\lambda_{0}$-Position. Die jeweils zwei horizontalen Striche links und rechts in jedem Spektrum markieren den betrachteten Ortsbereich. Der zeitliche Abstand zwischen den Spektren beträgt $36 \mathrm{~s}$. 


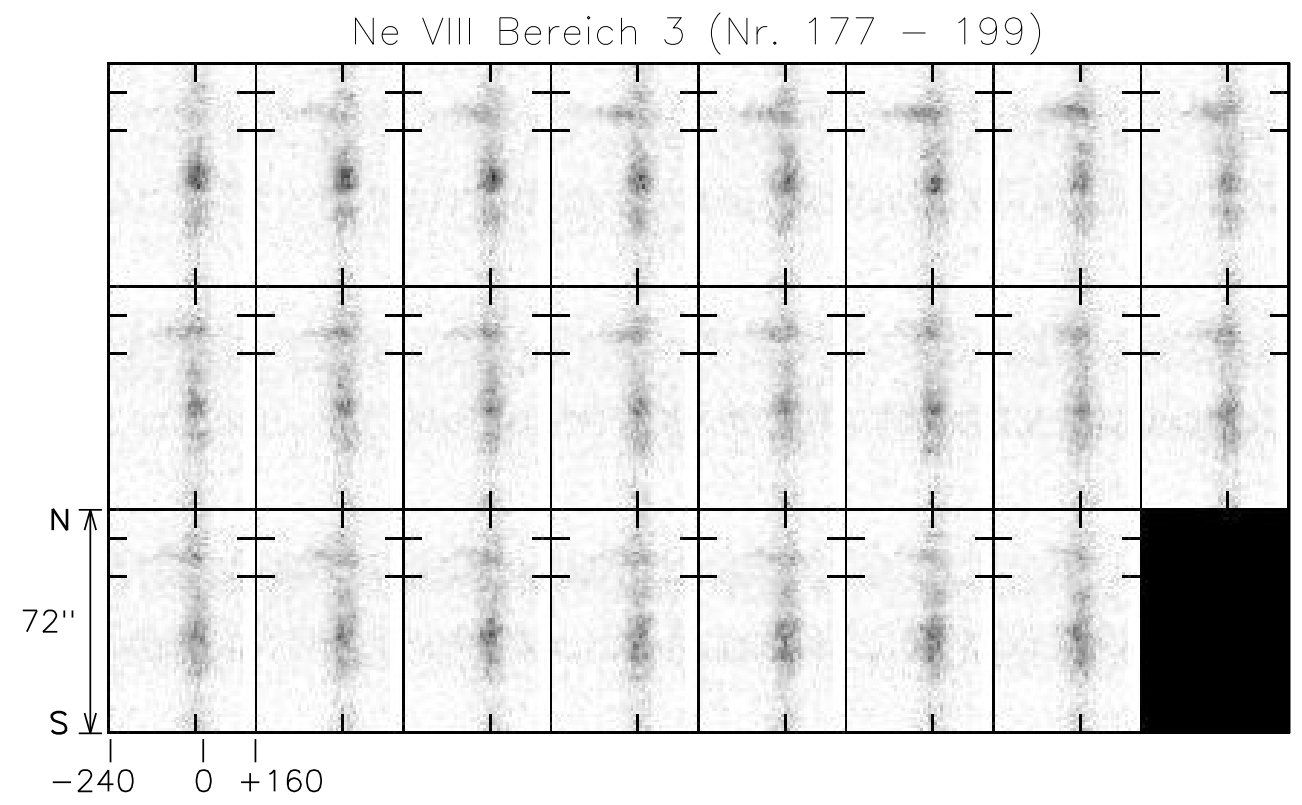

Geschwindigkeit in $\mathrm{km} / \mathrm{s}$

Abbildung 11.22: Spektren der Ne viII-Linie aus dem Bereich $3(\Delta \lambda \approx 0,21 \mathrm{~nm}, \Delta x \approx$ $\left.72^{\prime \prime}\right)$. Die kleinen vertikalen Striche oben und unten in jedem Spektrum markieren die $\lambda_{0}$-Position. Die jeweils zwei horizontalen Striche links und rechts in jedem Spektrum markieren den betrachteten Ortsbereich. Der zeitliche Abstand zwischen den Spektren beträgt 36s.

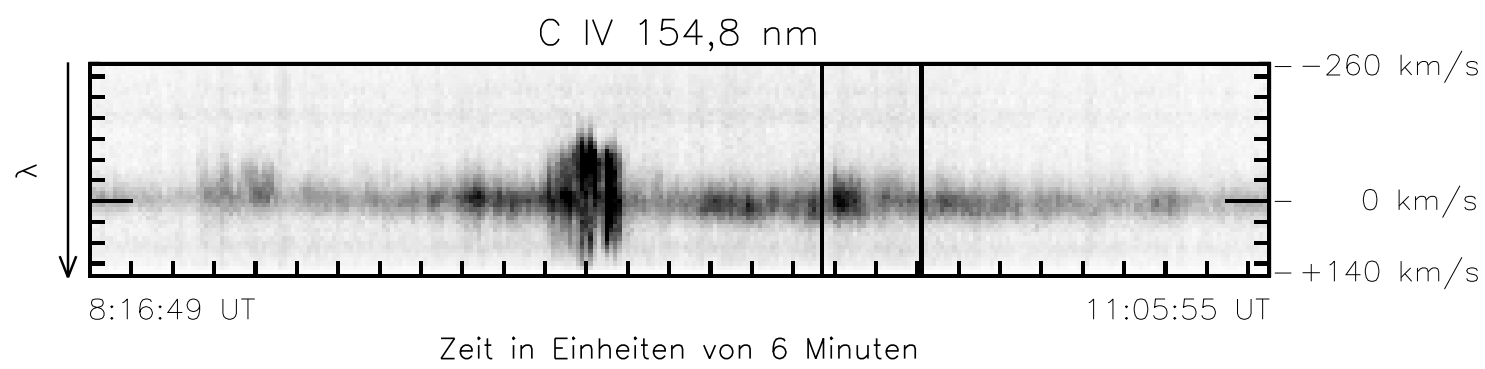

Abbildung 11.23: $\lambda t$-Diagramm C IV-Linie aus dem Bereich 3. Die zwei schwarzen vertikalen Linien grenzen den betrachteten Bereich zeitlich ein. Die Einheit auf der Geschwindigkeitsachse beträgt $\sim 40 \mathrm{~km} / \mathrm{s}$. 


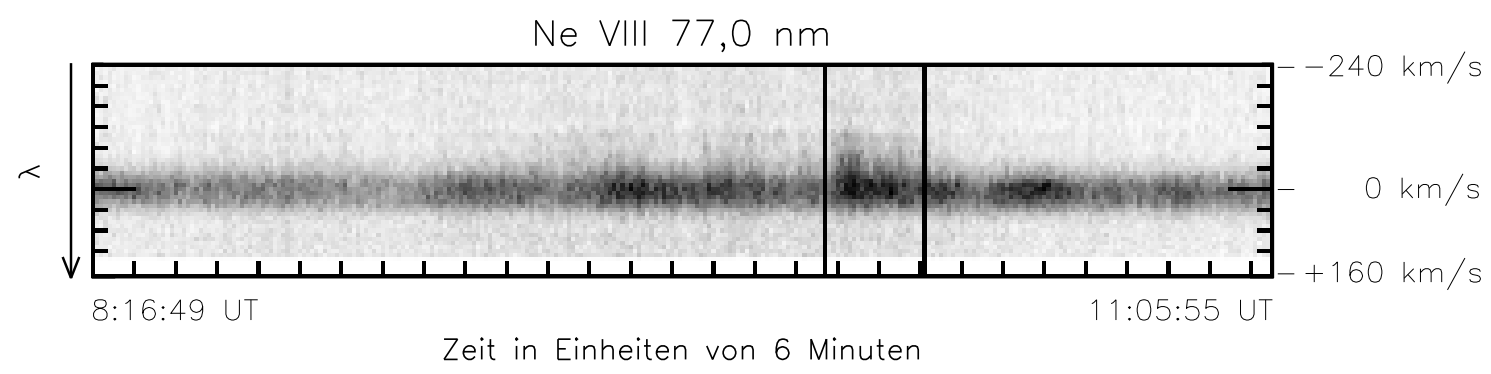

Abbildung 11.24: $\lambda t$-Diagramm Ne viII-Linie aus dem Bereich 3. Die zwei schwarzen vertikalen Linien grenzen den betrachteten Bereich zeitlich ein. Die Einheit auf der Geschwindigkeitsachse beträgt $\sim 40 \mathrm{~km} / \mathrm{s}$.
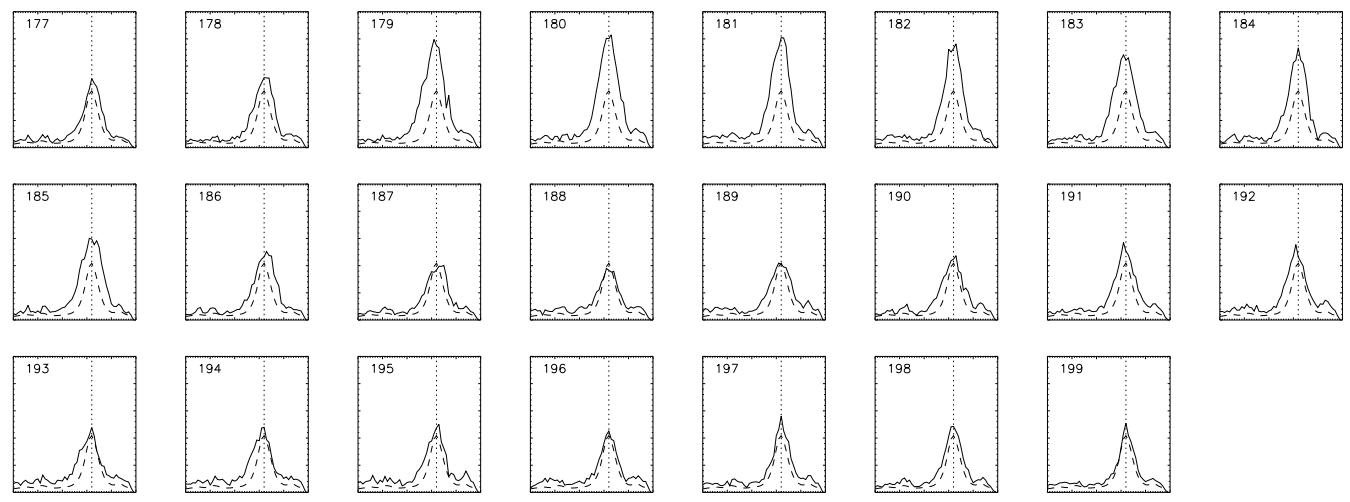

Abbildung 11.25: Über den betrachteten Ortsbereich gemittelte C IV-Spektren aus dem betrachteten Zeitraum. Die Intensität ist in relativen Einheiten gegen die Wellenlänge aufgetragen ( $\lambda$ steigt nach rechts an). Die vertikale, gepunktete Linie markiert die $\lambda_{0}$-Position, und das gestrichelte Profil gibt den gemittelten Spektrumsausschnitt wieder. 

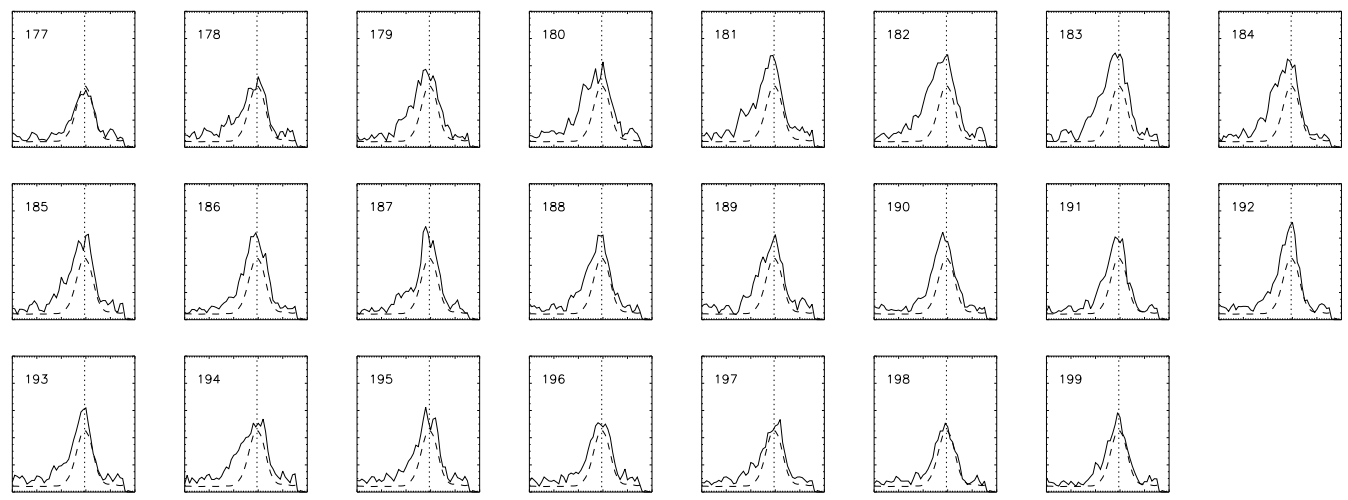

Abbildung 11.26: Über den betrachteten Ortsbereich gemittelte Ne VIII-Spektren aus dem betrachteten Zeitraum. Die Intensität ist in relativen Einheiten gegen die Wellenlänge aufgetragen ( $\lambda$ steigt nach rechts an). Die vertikale, gepunktete Linie markiert die $\lambda_{0}$-Position, und das gestrichelte Profil gibt den gemittelten Spektrumsausschnitt wieder. 


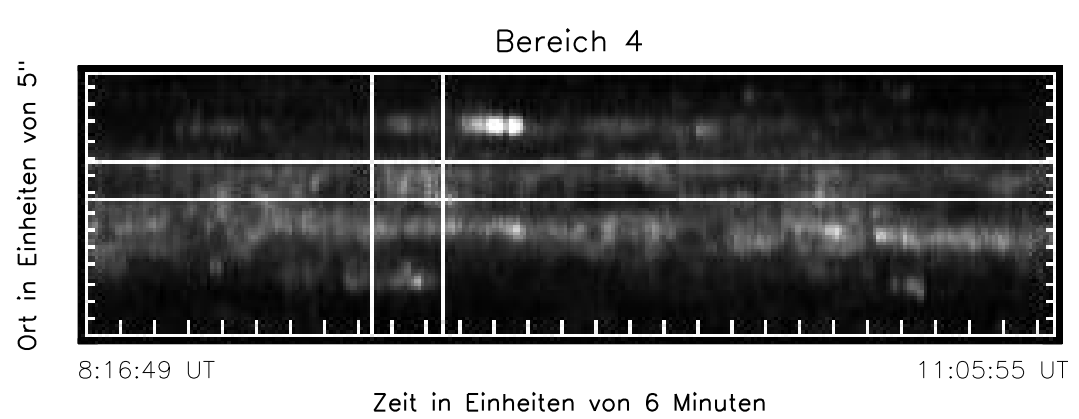

Abbildung 11.27: Bereich Nr. 4 auf dem Hintergrund des C IV-Diagramms.

\subsection{Bereich 4}

Der Bereich 4 liegt etwa mittig zwischen den Gebieten unterschiedlicher Polarität. Er ist $9,{ }^{\prime \prime} 5 \times 720 \mathrm{~s}$ groß. Es ist in diesem Bereich kein nennenswerter magnetischer Fluß nachweisbar (vgl. Abb. 11.1). Auffällig ist die kurzzeitige Aufhellung von $\sim 4$ Minuten Dauer in der Ne vin-Linie mit einem Maximum bei Nr. 93 in Abb. 11.33 bzw. 11.29. Man erkennt sie auch im $\lambda t$-Diagramm Abb. 11.31. Im $\lambda t$-Diagramm Abb. 11.30 der C IVLinie finden sich über das ganze betrachtete Intervall vier starke Aufhellungen (explosive Ereignisse) mit relativen Maxima bei Nr. 88, 92/93, 99 und 102 in Abb. 11.32 bzw. 11.28. 


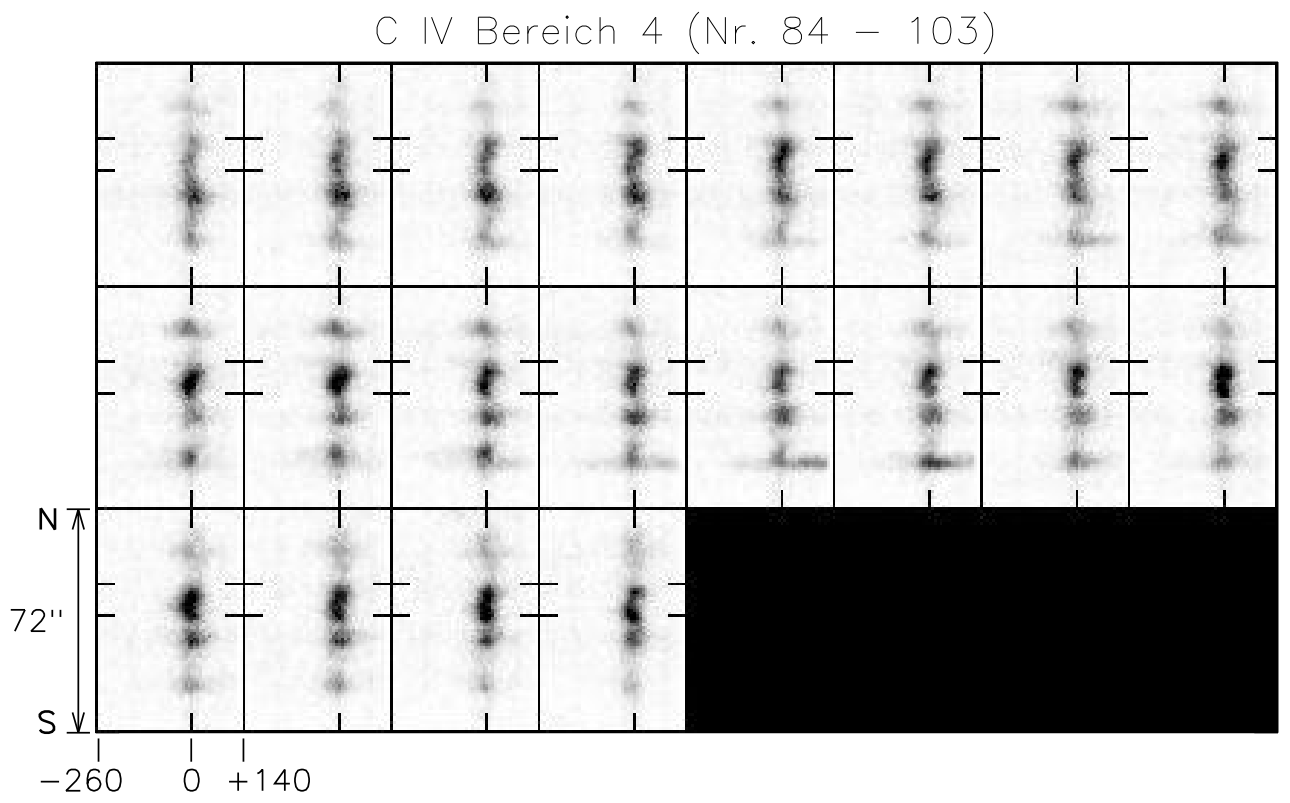

Geschwindigkeit in km/s

Abbildung 11.28: Spektren der C IV-Linie aus dem Bereich $4(\Delta \lambda \approx 0,21 \mathrm{~nm}, \Delta x \approx$ $\left.72^{\prime \prime}\right)$. Die kleinen vertikalen Striche oben und unten in jedem Spektrum markieren die $\lambda_{0}$-Position. Die jeweils zwei horizontalen Striche links und rechts in jedem Spektrum markieren den betrachteten Ortsbereich. Der zeitliche Abstand zwischen den Spektren beträgt $36 \mathrm{~s}$. 


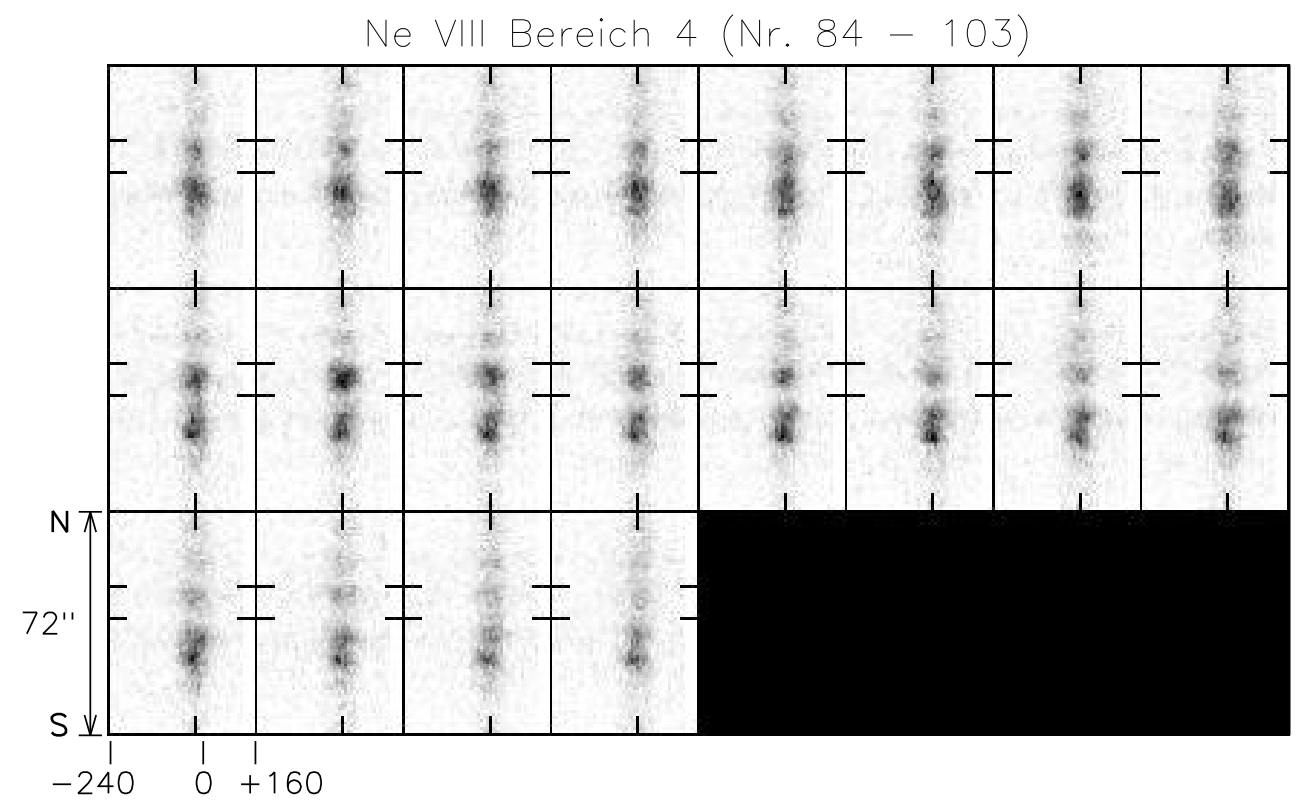

Geschwindigkeit in $\mathrm{km} / \mathrm{s}$

Abbildung 11.29: Spektren der Ne viII-Linie aus dem Bereich $4(\Delta \lambda \approx 0,21 \mathrm{~nm}, \Delta x \approx$ $\left.72^{\prime \prime}\right)$. Die kleinen vertikalen Striche oben und unten in jedem Spektrum markieren die $\lambda_{0}$-Position. Die jeweils zwei horizontalen Striche links und rechts in jedem Spektrum markieren den betrachteten Ortsbereich. Der zeitliche Abstand zwischen den Spektren beträgt 36s.

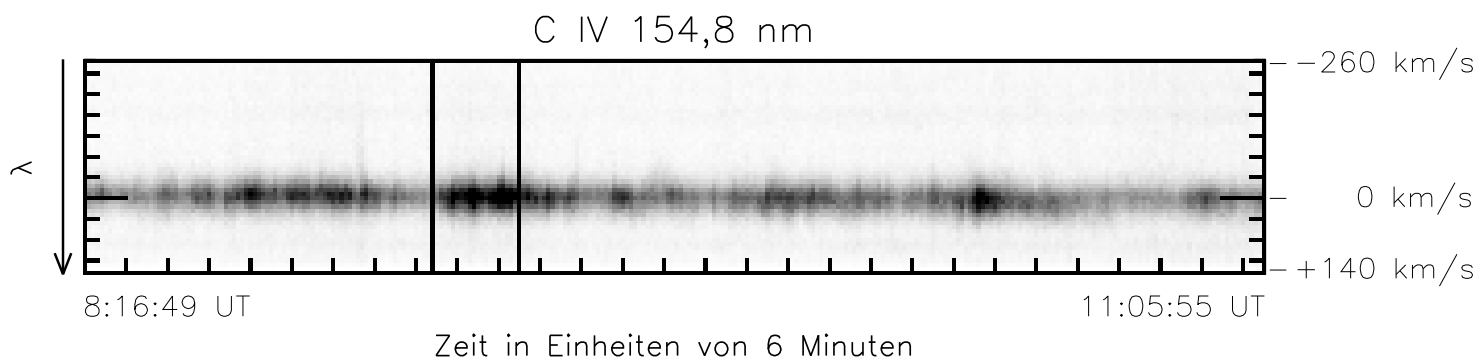

Abbildung 11.30: $\lambda t$-Diagramm C IV-Linie aus dem Bereich 4. Die zwei schwarzen vertikalen Linien grenzen den betrachteten Bereich zeitlich ein. Die Einheit auf der Geschwindigkeitsachse beträgt $\sim 40 \mathrm{~km} / \mathrm{s}$. 


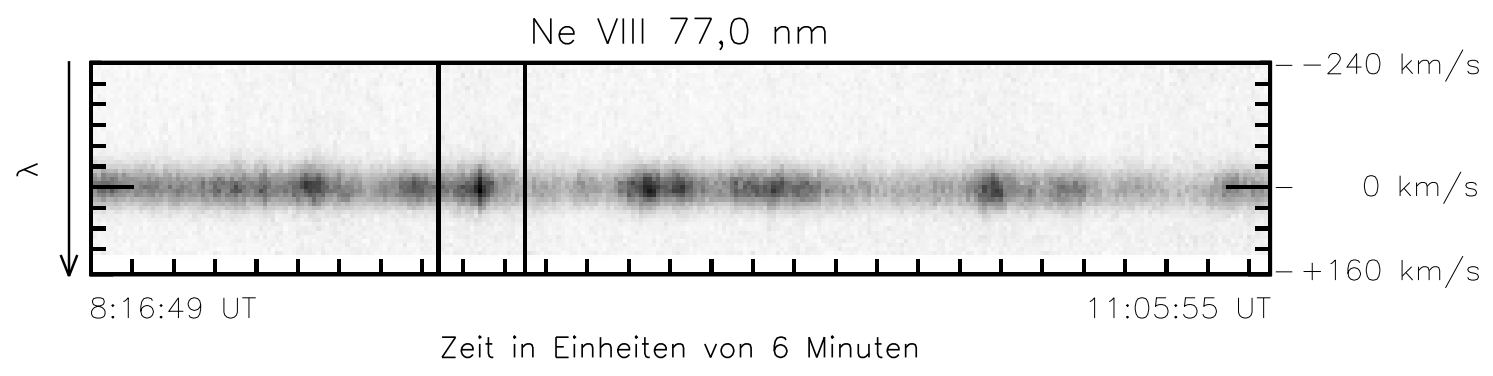

Abbildung 11.31: $\lambda t$-Diagramm Ne viII-Linie aus dem Bereich 4. Die zwei schwarzen vertikalen Linien grenzen den betrachteten Bereich zeitlich ein. Die Einheit auf der Geschwindigkeitsachse beträgt $\sim 40 \mathrm{~km} / \mathrm{s}$.
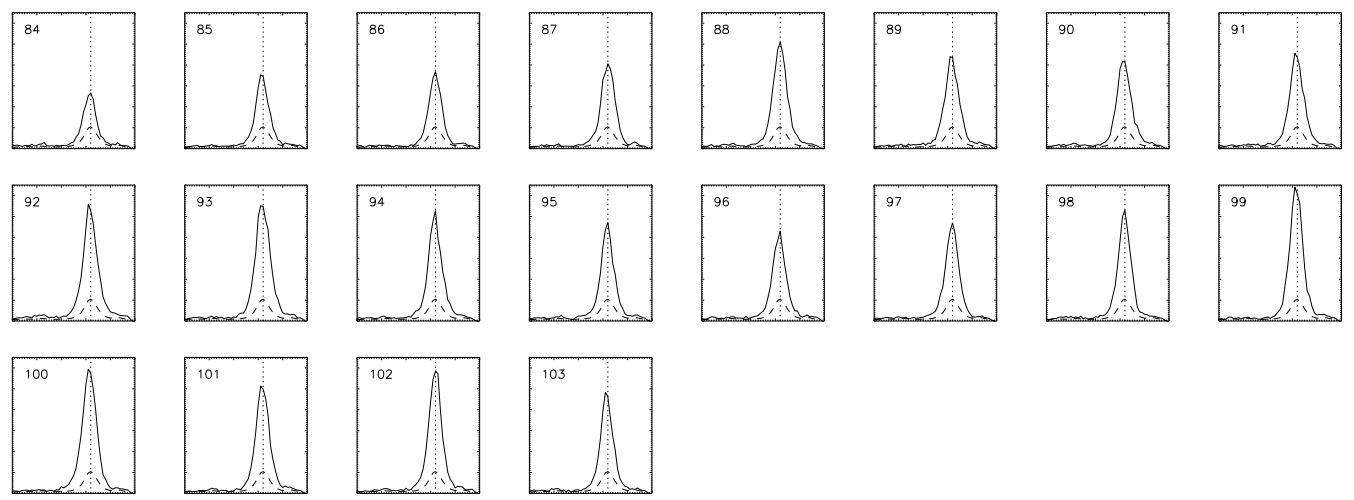

Abbildung 11.32: Über den betrachteten Ortsbereich gemittelte C IV-Spektren aus dem betrachteten Zeitraum. Die Intensität ist in relativen Einheiten gegen die Wellenlänge aufgetragen ( $\lambda$ steigt nach rechts an). Die vertikale, gepunktete Linie markiert die $\lambda_{0}$-Position, und das gestrichelte Profil gibt den gemittelten Spektrumsausschnitt wieder. 

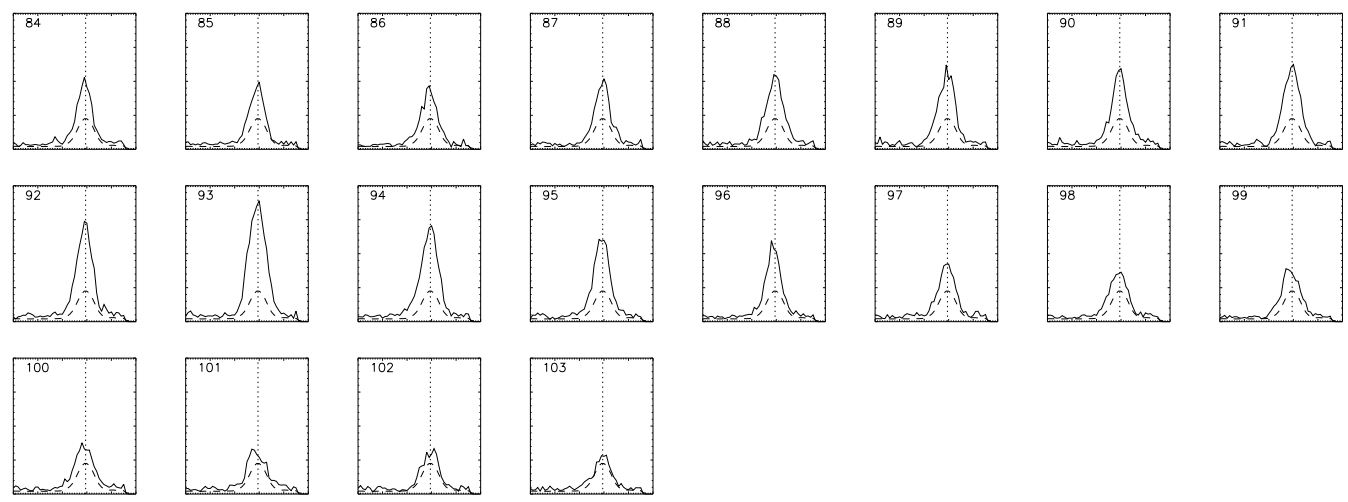

Abbildung 11.33: Über den betrachteten Ortsbereich gemittelte Ne VIII-Spektren aus dem betrachteten Zeitraum. Die Intensität ist in relativen Einheiten gegen die Wellenlänge aufgetragen ( $\lambda$ steigt nach rechts an). Die vertikale, gepunktete Linie markiert die $\lambda_{0}$-Position, und das gestrichelte Profil gibt den gemittelten Spektrumsausschnitt wieder. 


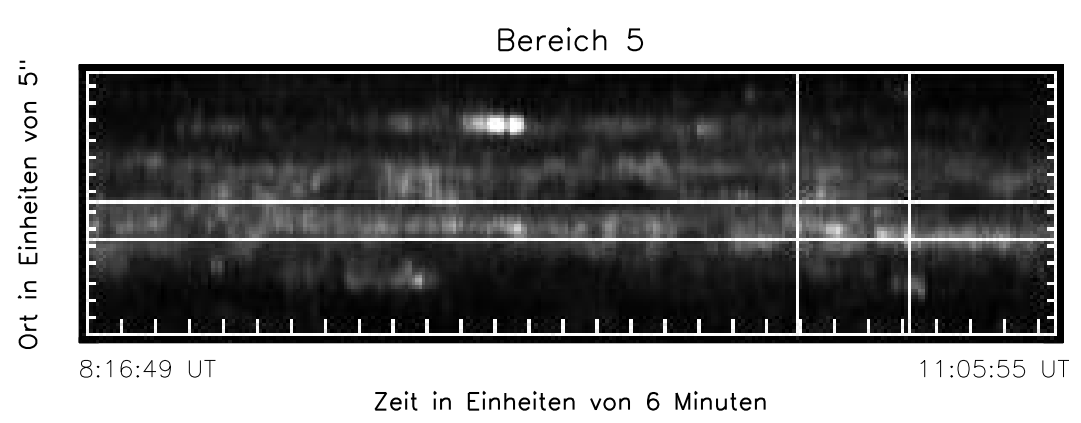

Abbildung 11.34: Bereich Nr. 5 auf dem Hintergrund des C IV-Diagramms.

\subsection{Bereich 5}

Der Bereich 5 liegt, genau wie Bereich 3, außerhalb des Zeitabschnittes, in dem Magnetogramme aufgenommen wurden. Er hat die Abmessungen 9," $5 \times 1152 \mathrm{~s}$ (19 min 12s). Dieser Bereich weist ein zweimaliges starkes Aufleuchten in der Ne VIII-Linie auf (Abb. 11.38). Ein stetiges Ansteigen der Intensität ist sichtbar von Nr. 211 in Abb. 11.36 und 11.40 bis zum Maximum bei Nr. 218 bis 220 und anschließendes Absinken mit lokalen Maxima bei Nr. 224 und 227. Ab Nr. 230 steigt die Intensität wieder bis zum Erreichen eines Maximums bei Nr. 236/237 an, um dann wieder abzufallen. Der erste Vorgang überstreicht ca. 12 Minuten, der zweite ca.5,5 Minuten. Die Entwicklung in der C IVLinie ist vergleichbar mit dem Intensitätsverlauf der Ne VIII-Linie (vgl. Abb. 11.37 und 11.38). Wenn auch der anfängliche Intensitätsanstieg nicht im gleichen Maß steil ausfällt, so finden sich doch auch in der C IV-Linie ein Maximum bei Nr. 219/220 (Abb. 11.39), ein Minimum bei Nr. 229/230. Ein weiteres Maximum erscheint bei Nr. 232 bis 234 (vgl. Abb. 11.35) also ca. 1 bis 2 Minuten vor dem der Ne viII-Linie. 


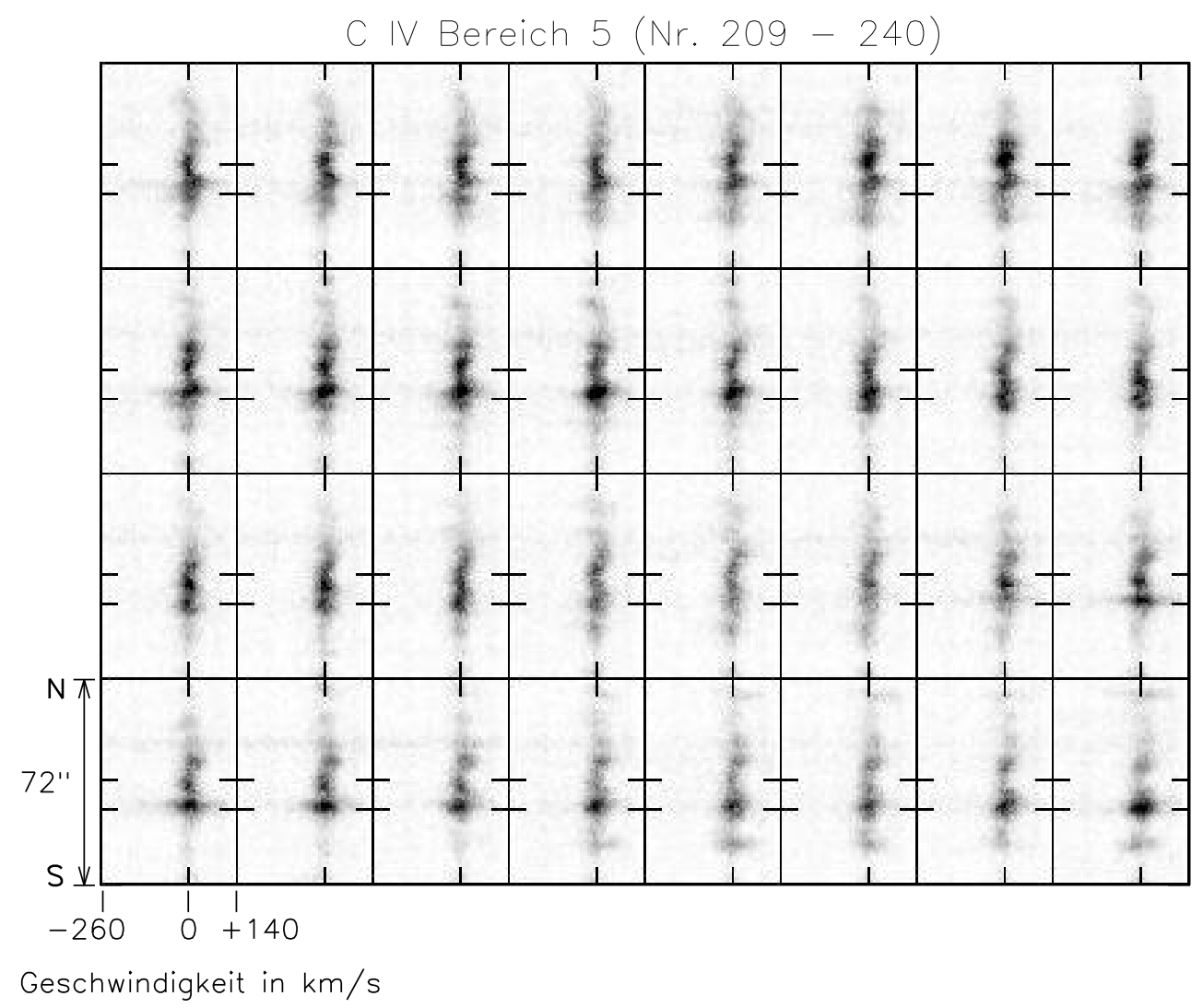

Abbildung 11.35: Spektren der CIV-Linie aus dem Bereich $5(\Delta \lambda \approx 0,21 \mathrm{~nm}, \Delta x \approx$ $\left.72^{\prime \prime}\right)$. Die kleinen vertikalen Striche oben und unten in jedem Spektrum markieren die $\lambda_{0}$-Position. Die jeweils zwei horizontalen Striche links und rechts in jedem Spektrum markieren den betrachteten Ortsbereich. Der zeitliche Abstand zwischen den Spektren beträgt $36 \mathrm{~s}$. 


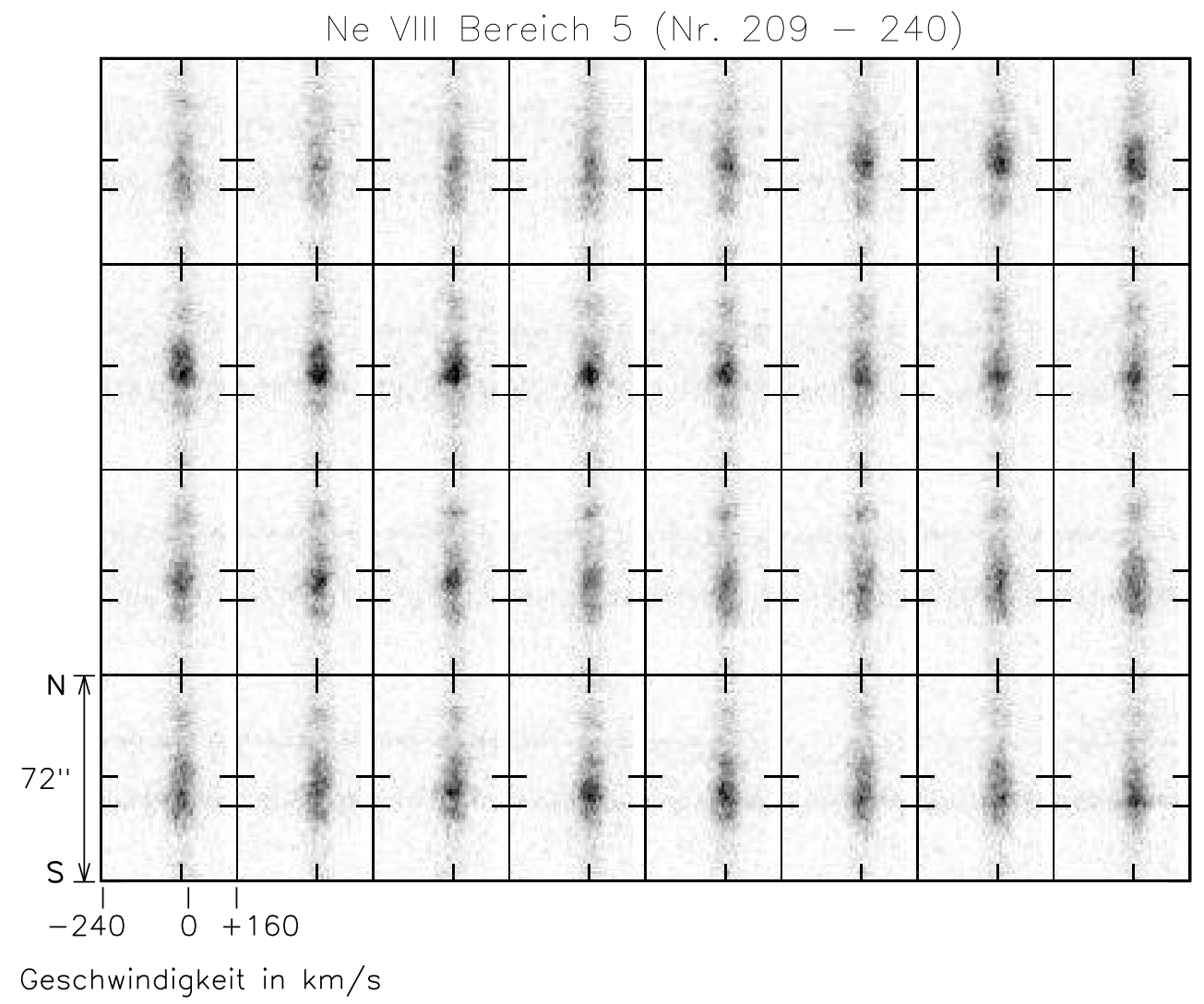

Abbildung 11.36: Spektren der Ne viII-Linie aus dem Bereich $5(\Delta \lambda \approx 0,21 \mathrm{~nm}, \Delta x \approx$ $\left.72^{\prime \prime}\right)$. Die kleinen vertikalen Striche oben und unten in jedem Spektrum markieren die $\lambda_{0}$-Position. Die jeweils zwei horizontalen Striche links und rechts in jedem Spektrum markieren den betrachteten Ortsbereich. Der zeitliche Abstand zwischen den Spektren beträgt $36 \mathrm{~s}$.

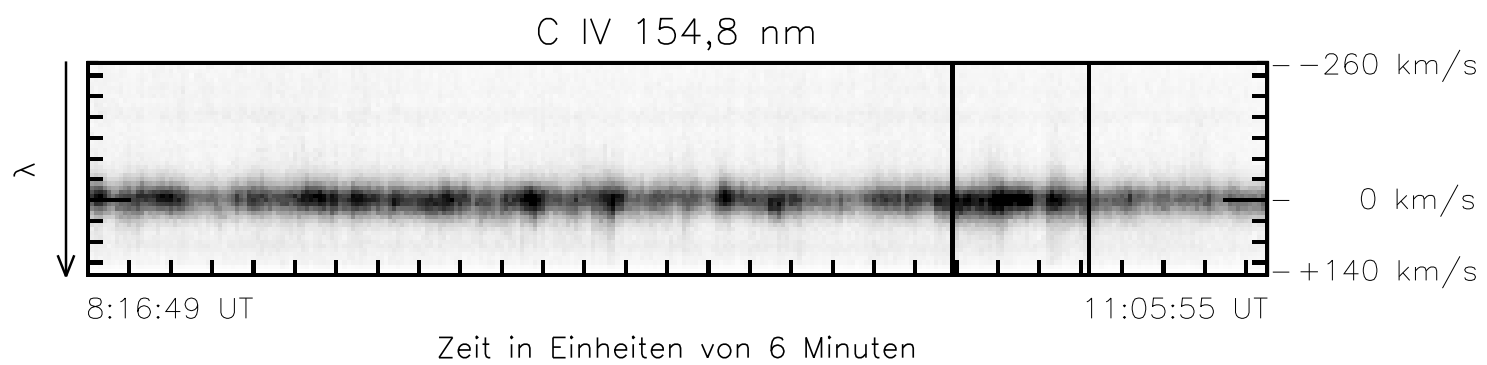

Abbildung 11.37: $\lambda t$-Diagramm C IV-Linie aus dem Bereich 5. Die zwei schwarzen vertikalen Linien grenzen den betrachteten Bereich zeitlich ein. Die Einheit auf der Geschwindigkeitsachse beträgt $\sim 40 \mathrm{~km} / \mathrm{s}$. 


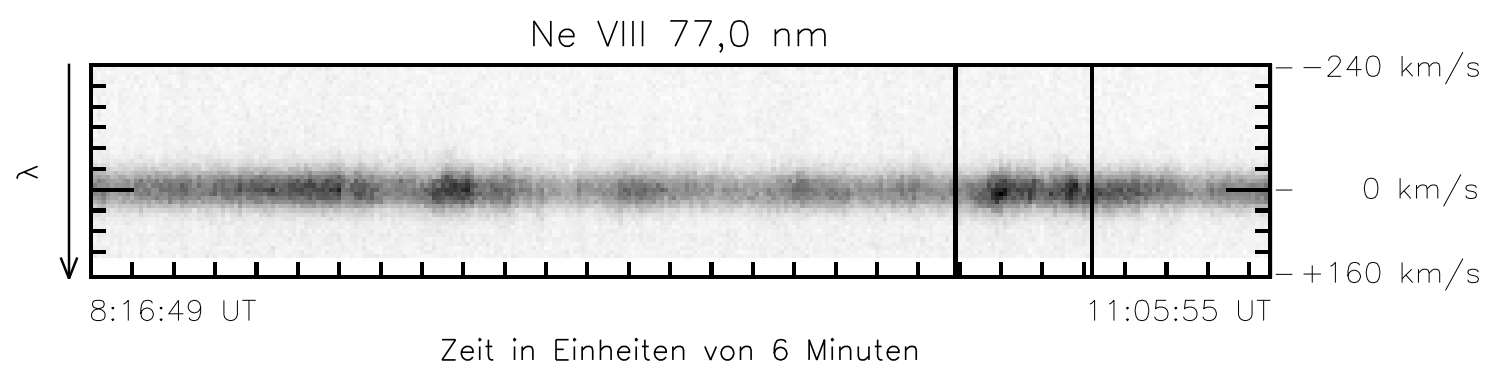

Abbildung 11.38: $\lambda t$-Diagramm Ne VIII-Linie aus dem Bereich 5. Die zwei schwarzen vertikalen Linien grenzen den betrachteten Bereich zeitlich ein. Die Einheit auf der Geschwindigkeitsachse beträgt $\sim 40 \mathrm{~km} / \mathrm{s}$.
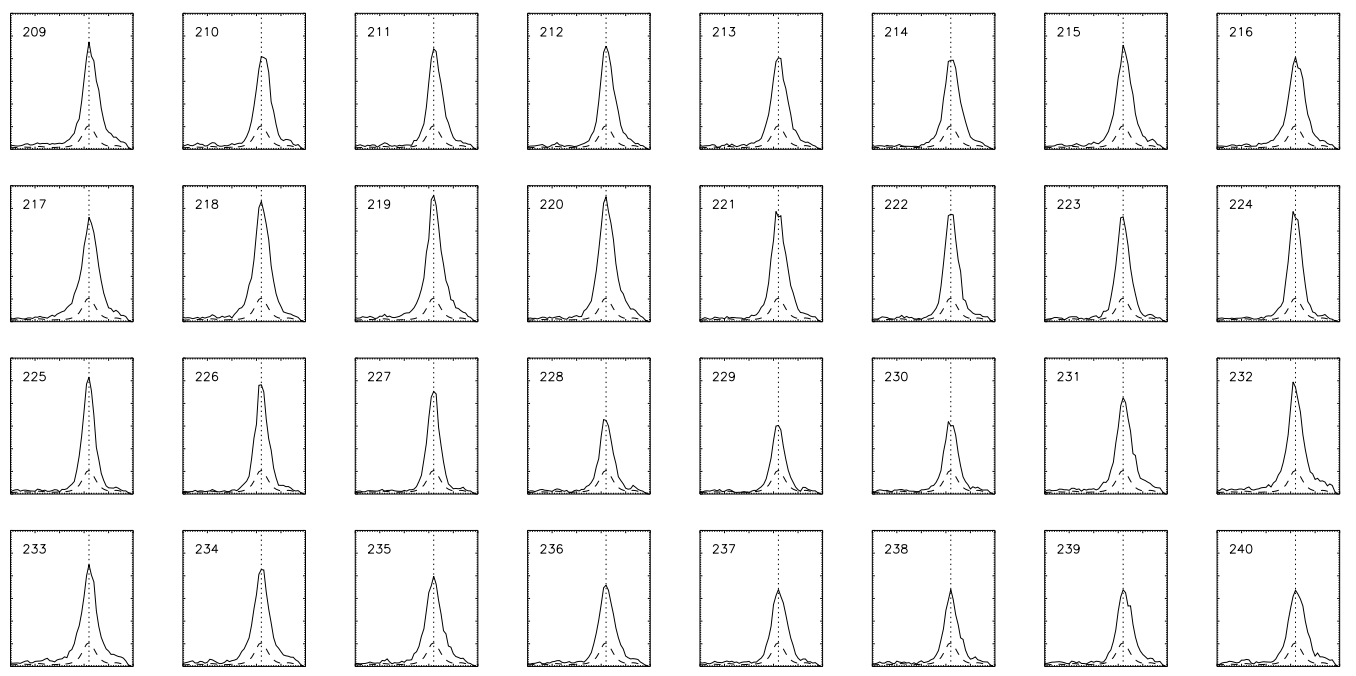

Abbildung 11.39: Über den betrachteten Ortsbereich gemittelte C IV-Spektren aus dem betrachteten Zeitraum. Die Intensität ist in relativen Einheiten gegen die Wellenlänge aufgetragen ( $\lambda$ steigt nach rechts an). Die vertikale, gepunktete Linie markiert die $\lambda_{0}$-Position, und das gestrichelte Profil gibt den gemittelten Spektrumsausschnitt wieder. 

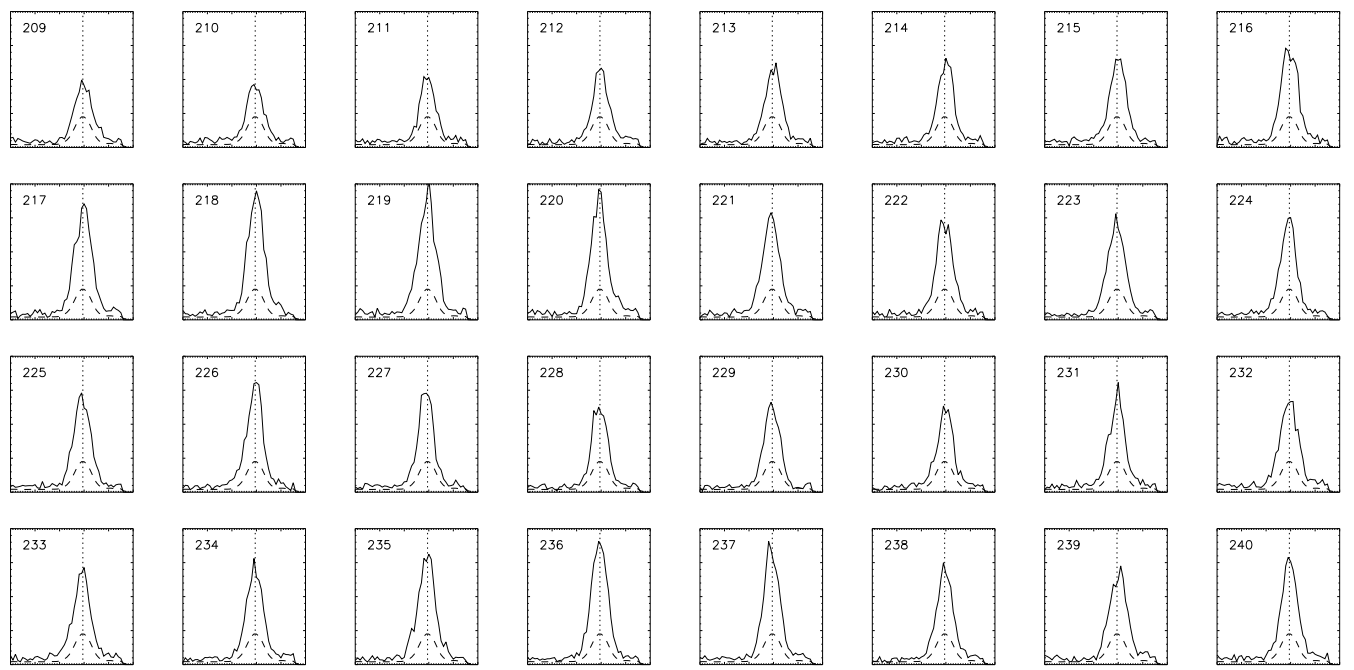

Abbildung 11.40: Über den betrachteten Ortsbereich gemittelte Ne VIII-Spektren aus dem betrachteten Zeitraum. Die Intensität ist in relativen Einheiten gegen die Wellenlänge aufgetragen ( $\lambda$ steigt nach rechts an). Die vertikale, gepunktete Linie markiert die $\lambda_{0}$-Position, und das gestrichelte Profil gibt den gemittelten Spektrumsausschnitt wieder. 


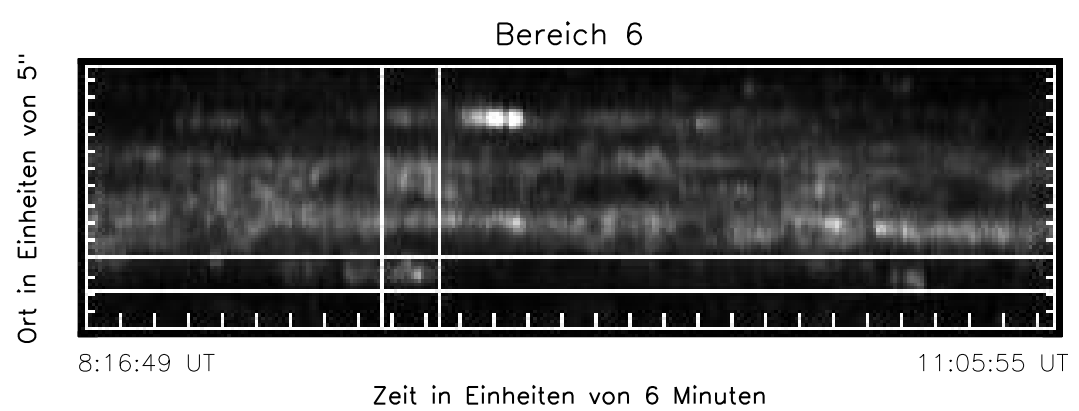

Abbildung 11.41: Bereich Nr. 6 auf dem Hintergrund des C IV-Diagramms.

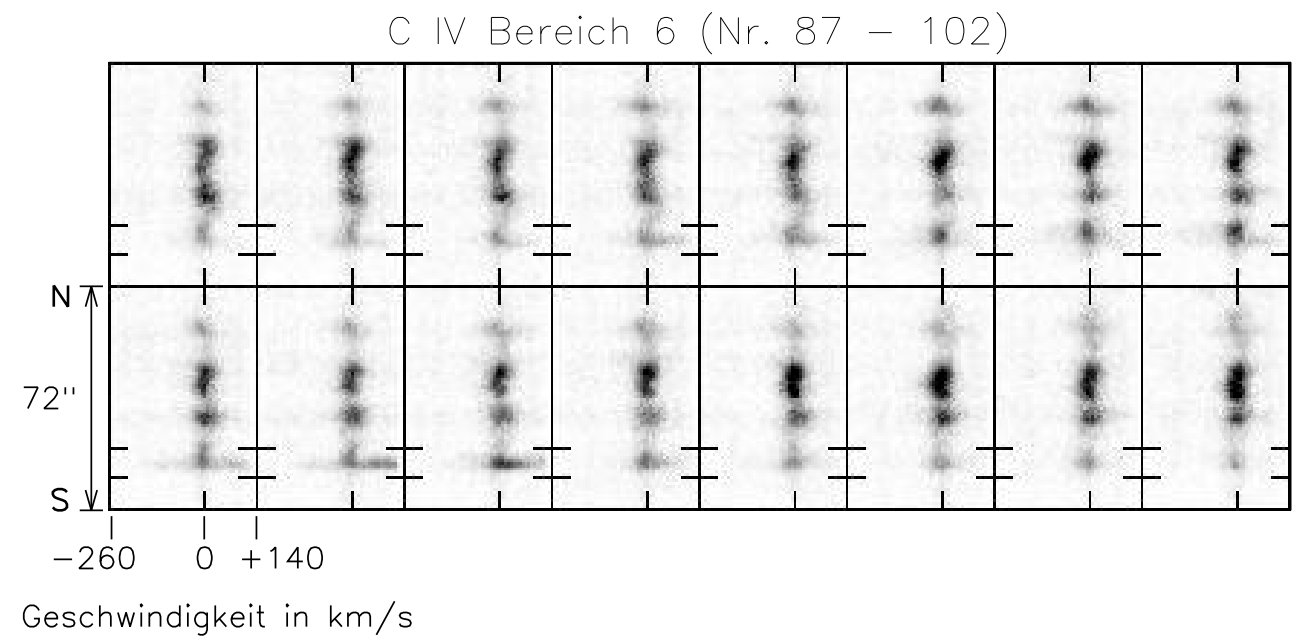

Abbildung 11.42: Spektren der C IV-Linie aus dem Bereich $6(\Delta \lambda \approx 0,21 \mathrm{~nm}, \Delta x \approx$ $\left.72^{\prime \prime}\right)$. Die kleinen vertikalen Striche oben und unten in jedem Spektrum markieren die $\lambda_{0}$-Position. Die jeweils zwei horizontalen Striche links und rechts in jedem Spektrum markieren den betrachteten Ortsbereich. Der zeitliche Abstand zwischen den Spektren beträgt $36 \mathrm{~s}$.

\subsection{Bereich 6}

Der Bereich 6 (Abb. 11.41) liegt südlich von der negativen Polarität in einem Gebiet, in dem kein signifikanter magnetischer Fluß nachgewiesen werden konnte (vgl. 11.1). Auffällig ist dieses Gebiet durch seine starke Aufhellung in der C IV-Linie bei gleichzeitiger, fast völliger Dunkelheit in der Ne viII-Linie (vgl. 11.1). Der Bereich 6 umfaßt $\sim 8^{\prime \prime}, 5$ in Ortsrichtung und $324 \mathrm{~s}$ ( 5 min $24 \mathrm{~s}$ ). Betrachtet man zunächst die Abb. 11.43, 11.45 und 11.47 der Ne VIII-Linie, so bestätigt sich das Bild, das man schon aufgrund des $x t$ Diagramms der Abb. 11.1 bekommen hatte. Die meiste Zeit liegen die Intensitäten der Ne VIII-Linie unterhalb des mittleren Profils (vgl. 11.47), in Abb. 11.43 ist der Bereich fast unsichtbar. Ganz anders erscheint der Bereich 6 im Lichte der C IV-Linie. Dort baut sich ab Nr. 88 in Abb. 11.46 eine zwar nur kurzzeitige aber gewaltige Rotverschiebung 


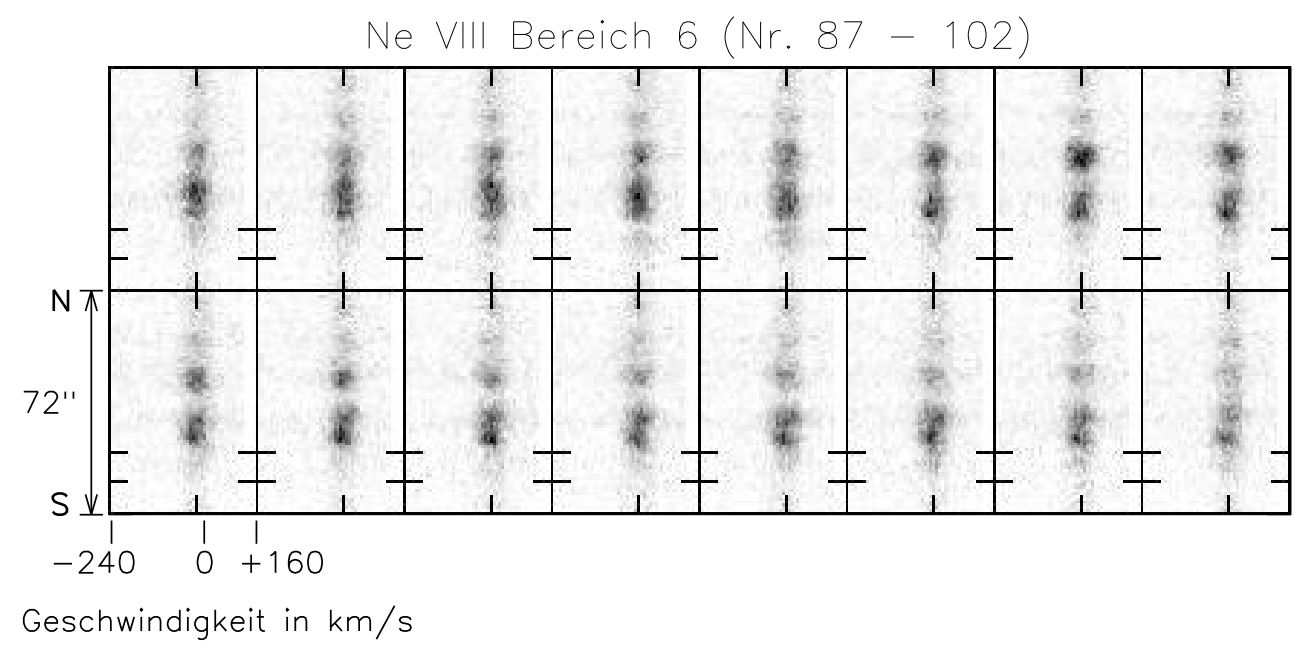

Abbildung 11.43: Spektren der Ne VIII-Linie aus dem Bereich $6(\Delta \lambda \approx 0,21 \mathrm{~nm}, \Delta x \approx$ $\left.72^{\prime \prime}\right)$. Die kleinen vertikalen Striche oben und unten in jedem Spektrum markieren die $\lambda_{0}$-Position. Die jeweils zwei horizontalen Striche links und rechts in jedem Spektrum markieren den betrachteten Ortsbereich. Der zeitliche Abstand zwischen den Spektren beträgt $36 \mathrm{~s}$.

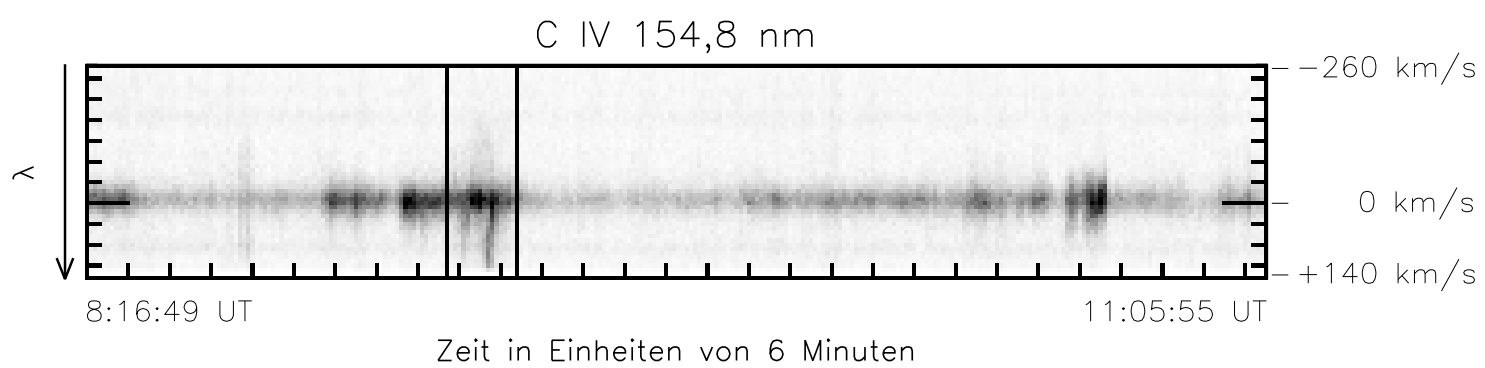

Abbildung 11.44: $\lambda t$-Diagramm C IV-Linie aus dem Bereich 6. Die zwei schwarzen vertikalen Linien grenzen den betrachteten Bereich zeitlich ein. Die Einheit auf der Geschwindigkeitsachse beträgt $\sim 40 \mathrm{~km} / \mathrm{s}$.

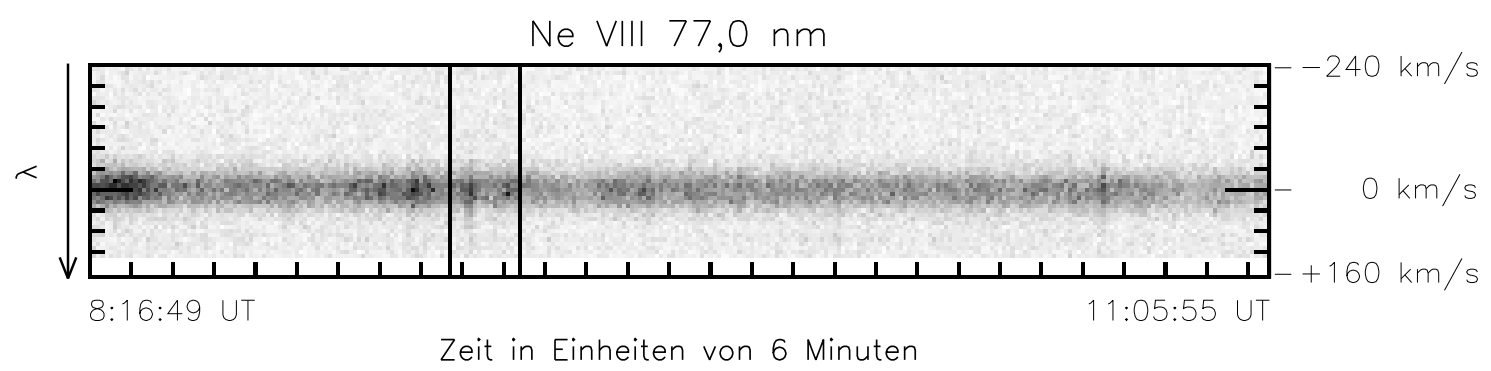

Abbildung 11.45: $\lambda$ t-Diagramm Ne viII-Linie aus dem Bereich 6. Die zwei schwarzen vertikalen Linien grenzen den betrachteten Bereich zeitlich ein. Die Einheit auf der Geschwindigkeitsachse beträgt $\sim 40 \mathrm{~km} / \mathrm{s}$. 

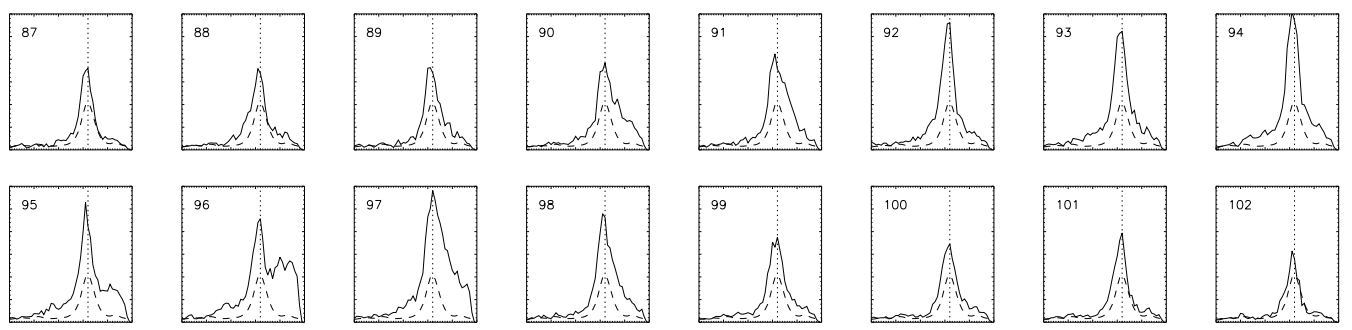

Abbildung 11.46: Über den betrachteten Ortsbereich gemittelte C IV-Spektren aus dem betrachteten Zeitraum. Die Intensität ist in relativen Einheiten gegen die Wellenlänge aufgetragen ( $\lambda$ steigt nach rechts an). Die vertikale, gepunktete Linie markiert die $\lambda_{0}$-Position, und das gestrichelte Profil gibt den gemittelten Spektrumsausschnitt wieder.
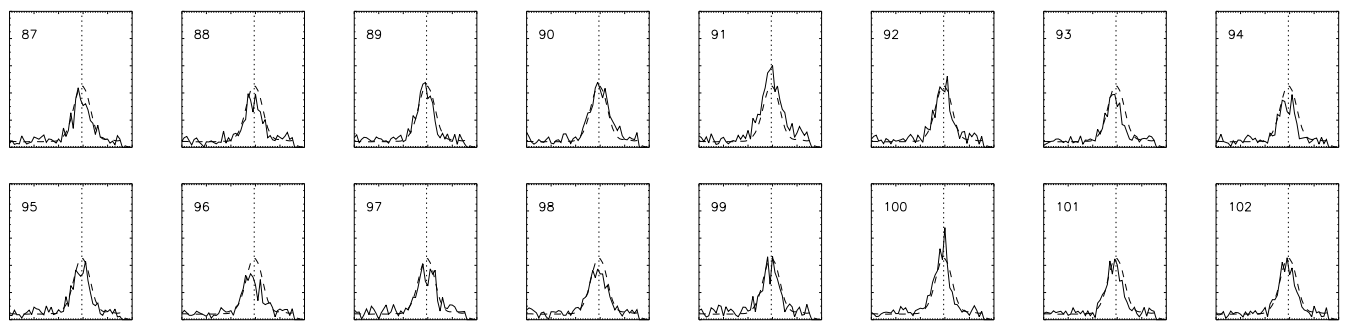

Abbildung 11.47: Über den betrachteten Ortsbereich gemittelte Ne VIII-Spektren aus dem betrachteten Zeitraum. Die Intensität ist in relativen Einheiten gegen die Wellenlänge aufgetragen ( $\lambda$ steigt nach rechts an). Die vertikale, gepunktete Linie markiert die $\lambda_{0}$-Position, und das gestrichelte Profil gibt den gemittelten Spektrumsausschnitt wieder. 


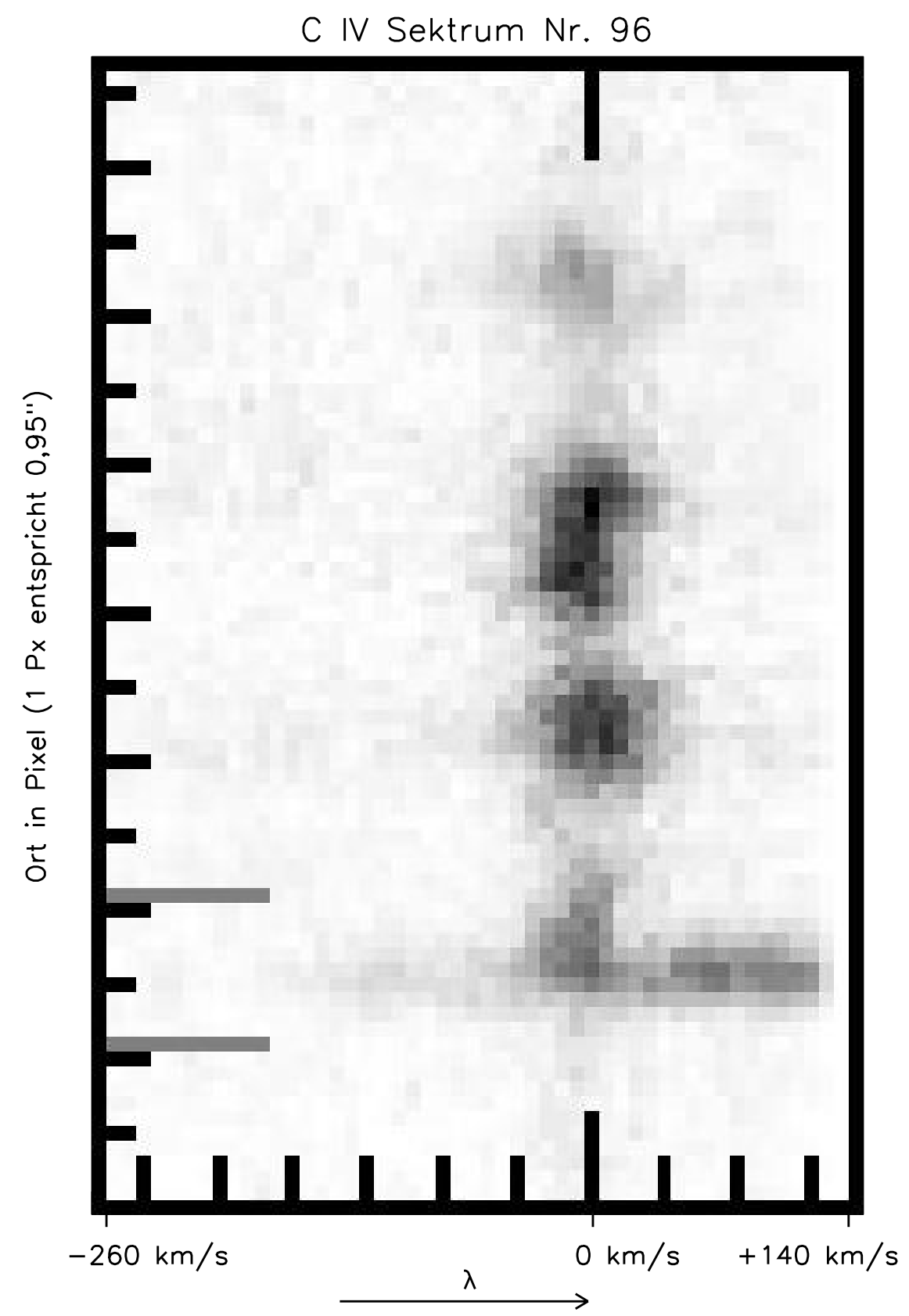

Pixel ( 1 Px entspricht 4,1866 pm bzw. $8 \mathrm{~km} / \mathrm{s}$ )

Abbildung 11.48: C IV-Spektrum Nr. $96\left(\Delta \lambda \approx 0,21 \mathrm{~nm}, \Delta x \approx 72^{\prime \prime}\right)$. Auf der Ortsachse wurde alle 5 Pixel eine Markierung gesetzt. Die Einheit auf der Geschwindigkeitsachse beträgt $\sim 40 \mathrm{~km} / \mathrm{s}$. Die kleinen vertikalen Striche oben und unten in jedem Spektrum markieren die $\lambda_{0}$-Position. Die jeweils zwei horizontalen Striche links und rechts in jedem Spektrum markieren den betrachteten Ortsbereich. 

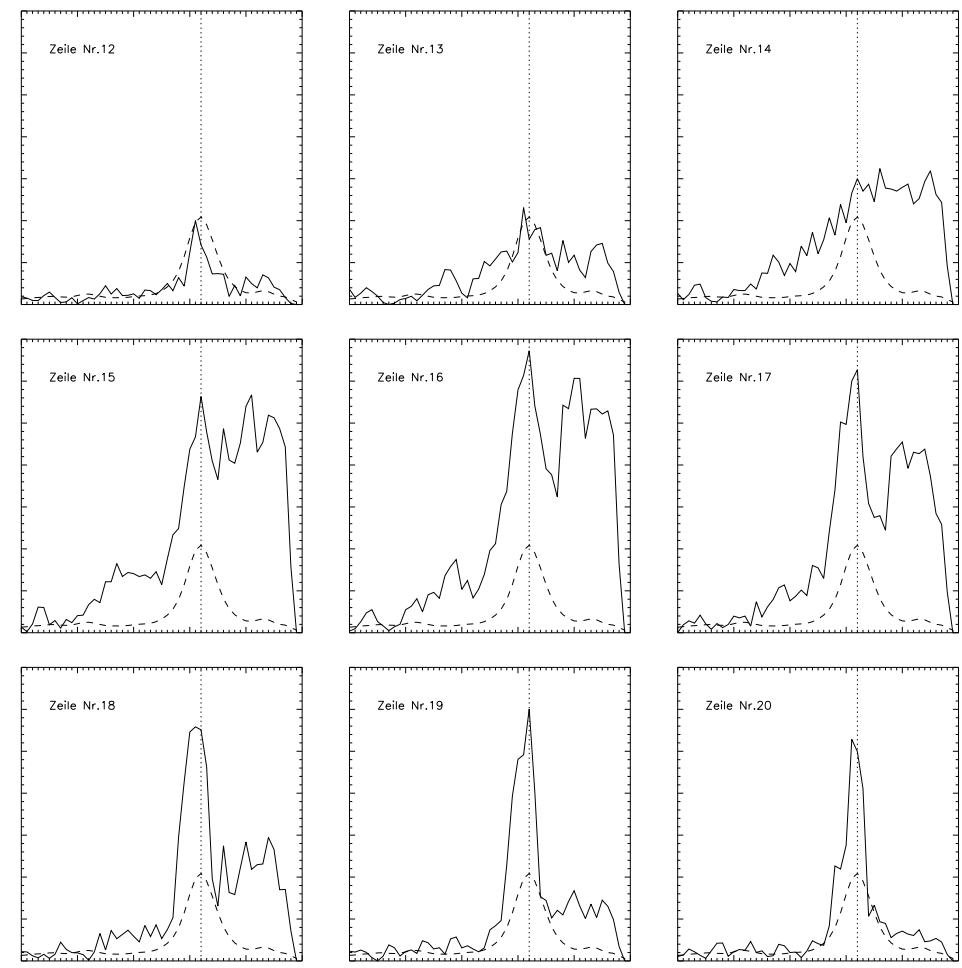

Abbildung 11.49: Aus dem markierten Bereich der Abb. 10.46 zeilenweise entnommene Spektrumsausschnitte. Die Intensität ist in relativen Einheiten gegen die Wellenlänge aufgetragen ( $\lambda$ steigt nach rechts an). Die vertikale, gepunktete Linie markiert die $\lambda_{0}$-Position, und das gestrichelte Profil gibt den gemittelten Spektrumsausschnitt wieder. 


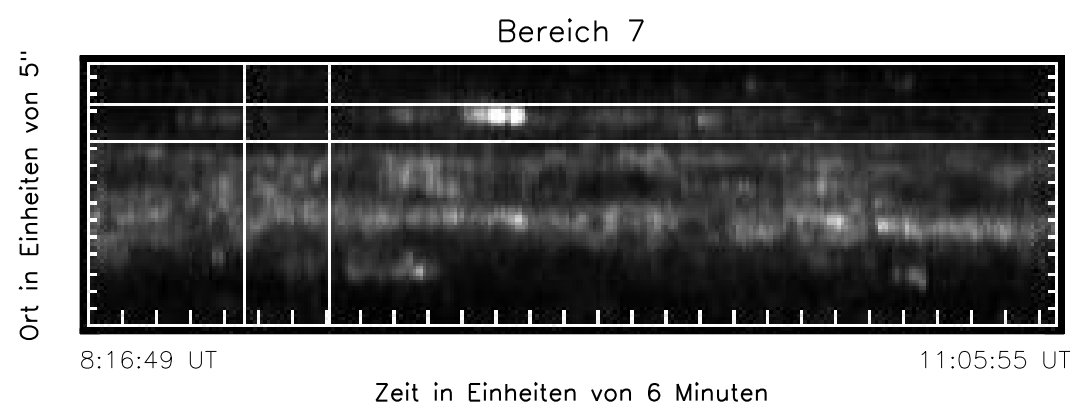

Abbildung 11.50: Bereich Nr. 7 auf dem Hintergrund des C IV-Diagramms.

auf.

Zunächst bildet sich, beginnend bei Nr. 89, in Nr. 90 eine starke Rotverschiebung, die dann wieder abnimmt (Nr. 91) und völlig verschwindet, bei gleichzeitig starkem Ansteigen der Zentrumsintensität (Nr. 92 bis 94). Die folgende sehr starke Rotverschiebung ist in Nr. 94 schon ansatzweise erkennbar. Sie erreicht nur 36 s danach (Nr. 96) ihr Maximum und ist dabei so stark, daß sie vom Detektorbereich nicht mehr vollständig aufgezeichnet werden kann (vgl. auch Abb. 11.48 und 11.49). Durch ein Anwachsen der Zentrumsintensität in Nr. 97 verschwindet die in Nr. 96 aufgetretene Aufspaltung der Linie. Über Nr. 98 verschwindet dann wieder die Rotverschiebung und bis Nr. 102 fällt auch die Zentrumsintensität wieder ungefähr auf mittleres Niveau (vgl. 11.44). Die ganze Erscheinung hat also eine zeitliche Ausdehnung von knapp 2 Minuten und bleibt räumlich auf $\sim 5^{\prime \prime}$ beschränkt (vgl. Abb. 11.48 und 11.49). Die dabei erreichten Geschwindigkeiten sind mit Sicherheit deutlich größer als $+140 \mathrm{~km} / \mathrm{s}$.

\subsection{Bereich 7}

Das Interesse an den drei folgenden Gebieten gründet sich auf die dort vorkommenden, relativ starken magnetischen Flüsse.

Der Bereich 7 (Abb. 11.50) umschließt den im xt-Diagramm Abb. 10.2 bzw. 11.1 stärksten magnetischen Fluß positiver Polarität. Dieser Bereich ist 9," $5 \times 864 \mathrm{~s}(14 \mathrm{~min} 24 \mathrm{~s})$ groß. Die Entwicklung des magnetischen Flusses ist in Abb. 11.51 wiedergegeben. Dem starken Fluß steht in beiden Linien, der Ne VIII- wie auch der C IV-Linie (vgl. die Spektren Abb. 11.52 und 11.53), das deutliche Nichtvorhandensein von Aufhellungen gegenüber. Die $\lambda t$-Diagramme der beiden Linien Abb. 11.54 und 11.55 weisen im betrachteten Bereich gradezu eine Lücke auf. Anhand der Abb. 11.56 und 11.57 erkennt man, daß die Intensitäten maximal das Niveau der gemittelten Bezugsspektren erreichen (gestricheltes Profil), häufig jedoch darunter liegen. 


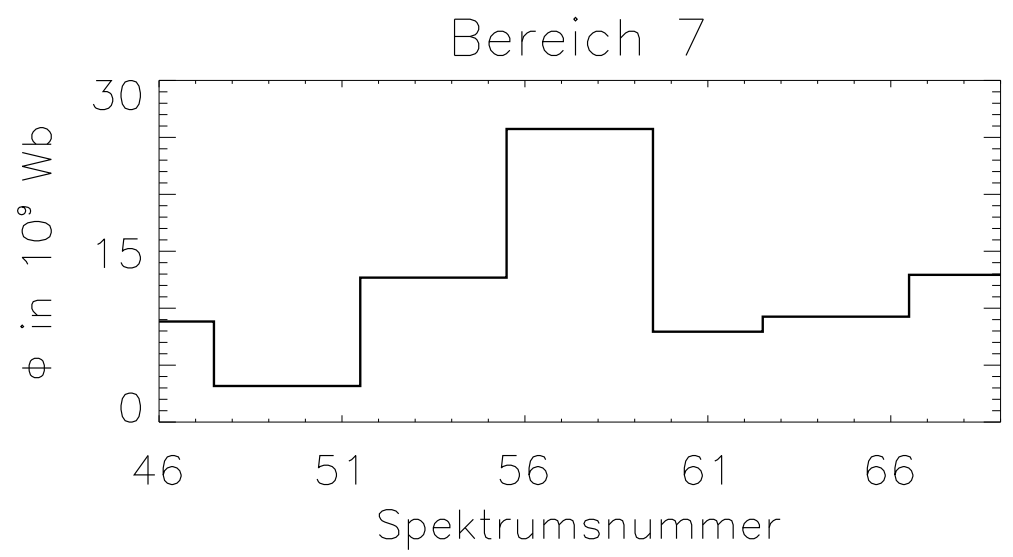

Abbildung 11.51: Über den betrachteten Ortsbereich integrierter magnetischer Fluß.

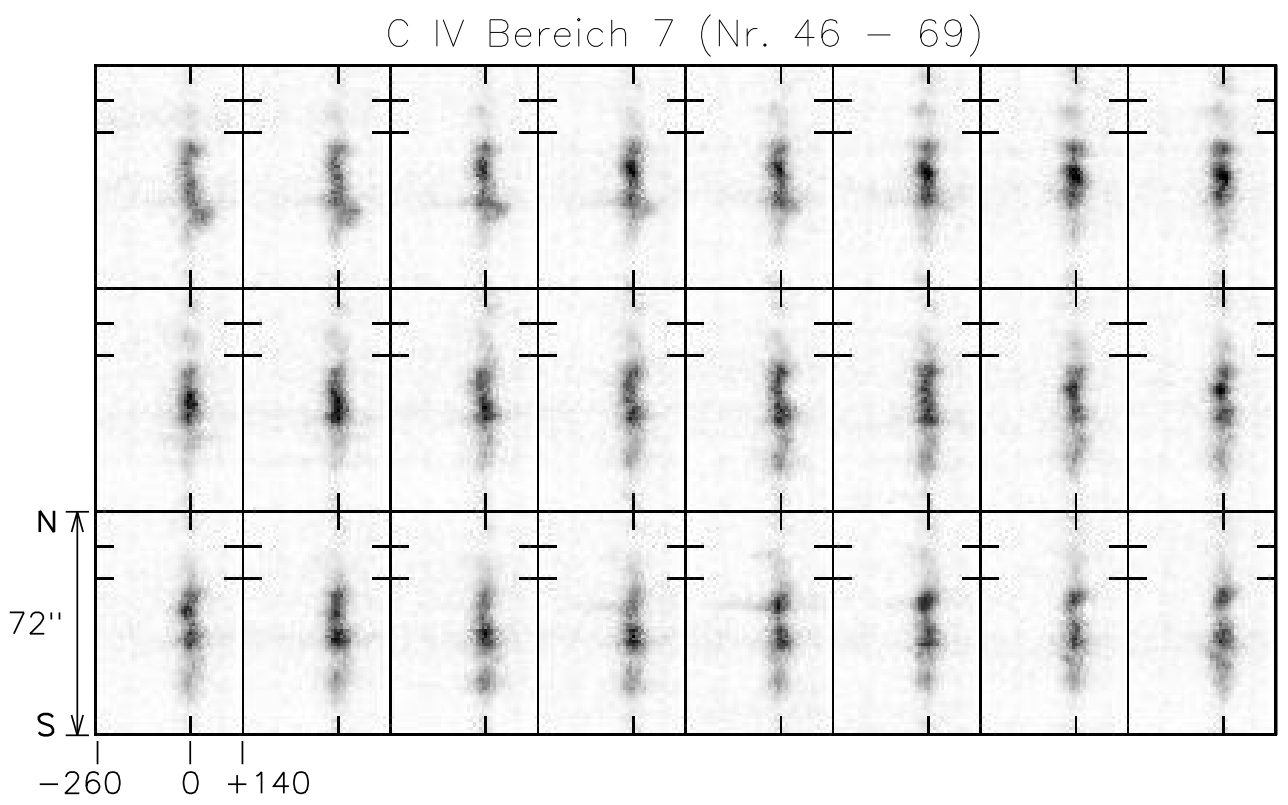

Geschwindigkeit in $\mathrm{km} / \mathrm{s}$

Abbildung 11.52: Spektren der C IV-Linie aus dem Bereich $7(\Delta \lambda \approx 0,21 \mathrm{~nm}, \Delta x \approx$ $\left.72^{\prime \prime}\right)$. Die kleinen vertikalen Striche oben und unten in jedem Spektrum markieren die $\lambda_{0}$-Position. Die jeweils zwei horizontalen Striche links und rechts in jedem Spektrum markieren den betrachteten Ortsbereich. Der zeitliche Abstand zwischen den Spektren beträgt $36 \mathrm{~s}$. 


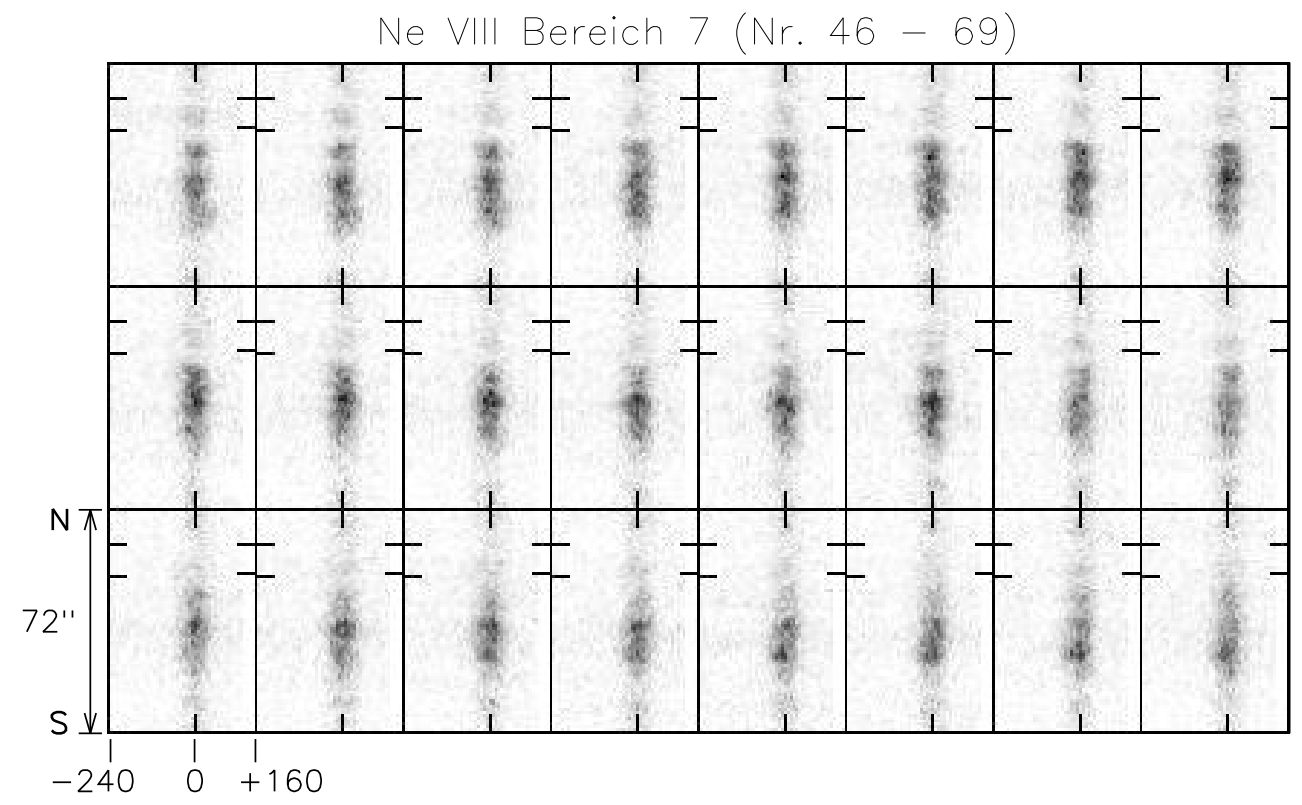

Geschwindigkeit in $\mathrm{km} / \mathrm{s}$

Abbildung 11.53: Spektren der Ne viII-Linie aus dem Bereich $7(\Delta \lambda \approx 0,21 \mathrm{~nm}, \Delta x \approx$ $\left.72^{\prime \prime}\right)$. Die kleinen vertikalen Striche oben und unten in jedem Spektrum markieren die $\lambda_{0}$-Position. Die jeweils zwei horizontalen Striche links und rechts in jedem Spektrum markieren den betrachteten Ortsbereich. Der zeitliche Abstand zwischen den Spektren beträgt 36s.

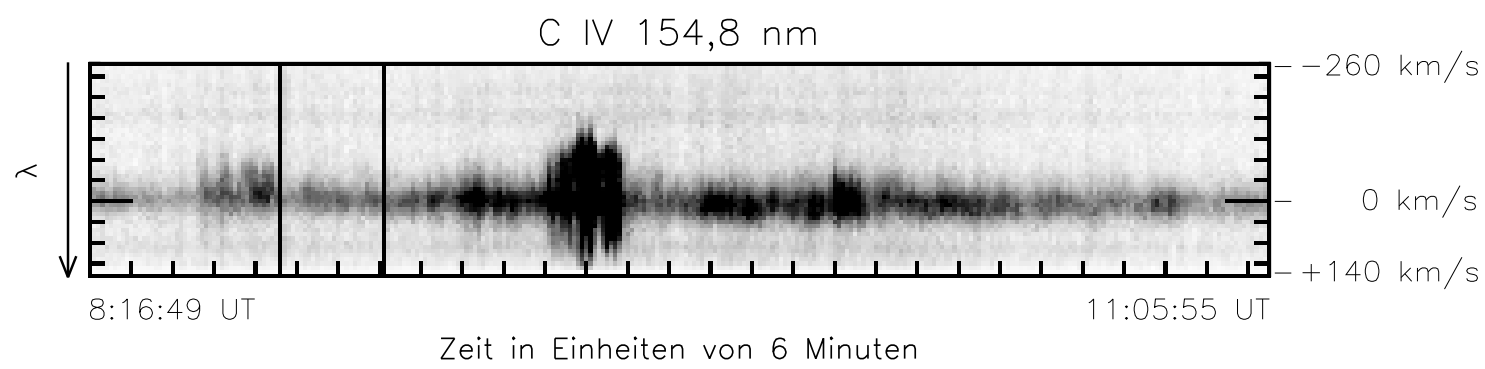

Abbildung 11.54: $\lambda t$-Diagramm C IV-Linie aus dem Bereich 7. Die zwei schwarzen vertikalen Linien grenzen den betrachteten Bereich zeitlich ein. Die Einheit auf der Geschwindigkeitsachse beträgt $\sim 40 \mathrm{~km} / \mathrm{s}$. 


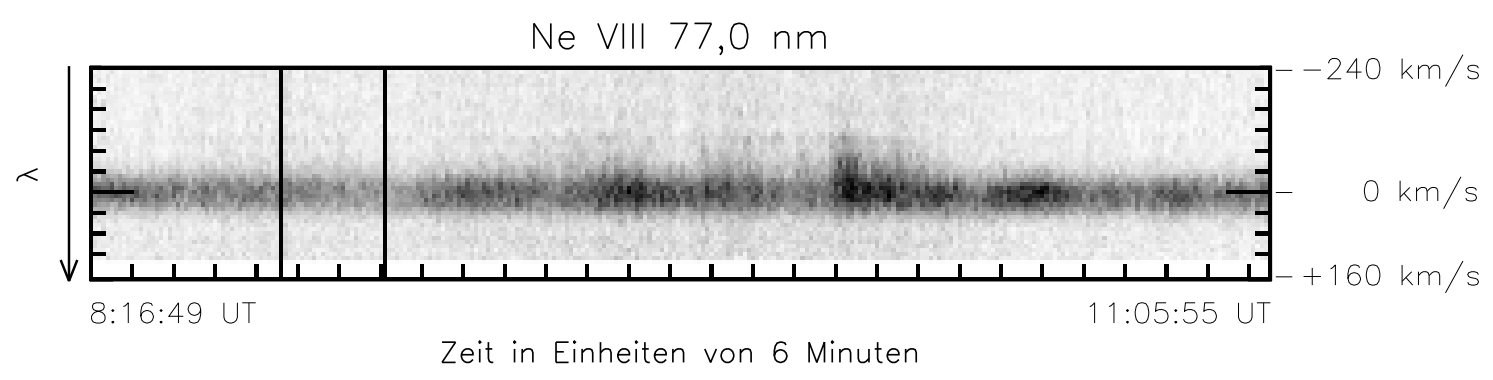

Abbildung 11.55: $\lambda t$-Diagramm Nevini-Linie aus dem Bereich 7. Die zwei schwarzen vertikalen Linien grenzen den betrachteten Bereich zeitlich ein. Die Einheit auf der Geschwindigkeitsachse beträgt $\sim 40 \mathrm{~km} / \mathrm{s}$.
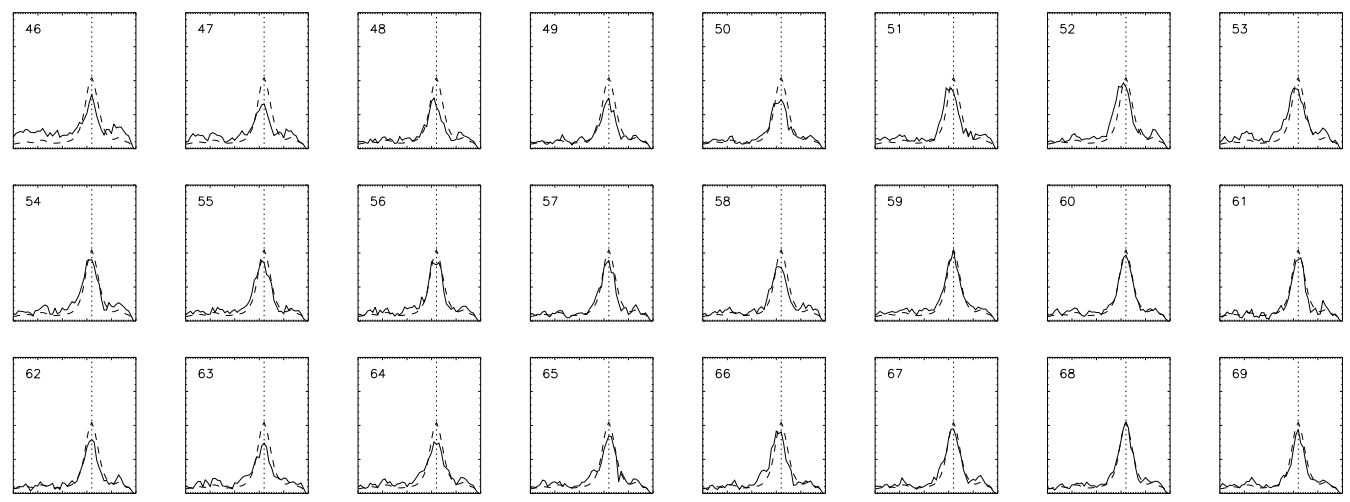

Abbildung 11.56: Über den betrachteten Ortsbereich gemittelte C IV-Spektren aus dem betrachteten Zeitraum. Die Intensität ist in relativen Einheiten gegen die Wellenlänge aufgetragen ( $\lambda$ steigt nach rechts an). Die vertikale, gepunktete Linie markiert die $\lambda_{0}$-Position, und das gestrichelte Profil gibt den gemittelten Spektrumsausschnitt wieder. 

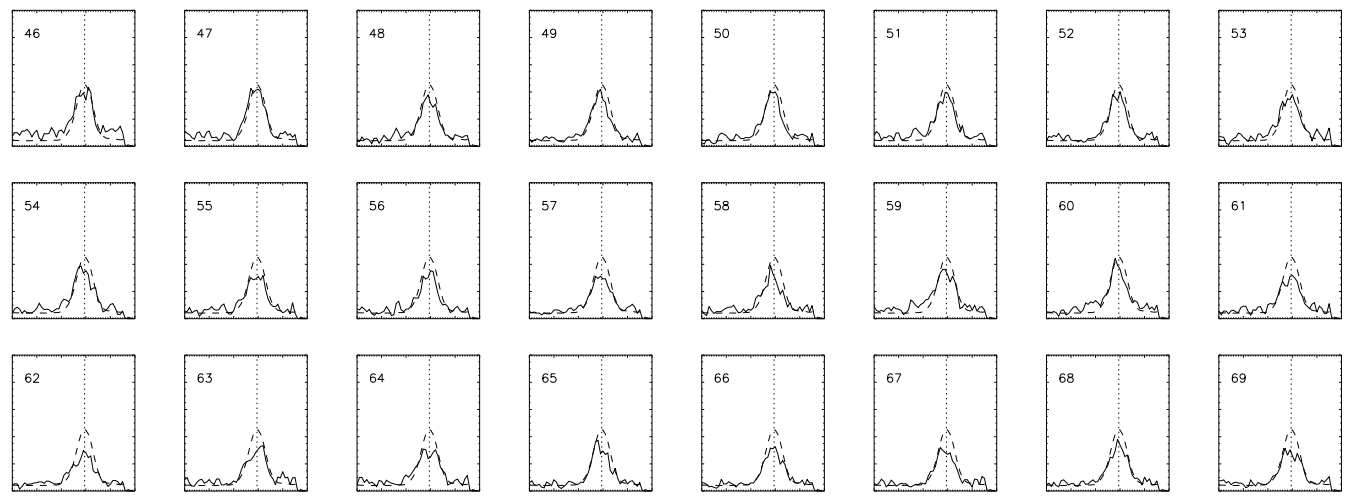

Abbildung 11.57: Über den betrachteten Ortsbereich gemittelte Ne VIII-Spektren aus dem betrachteten Zeitraum. Die Intensität ist in relativen Einheiten gegen die Wellenlänge aufgetragen ( $\lambda$ steigt nach rechts an). Die vertikale, gepunktete Linie markiert die $\lambda_{0}$-Position, und das gestrichelte Profil gibt den gemittelten Spektrumsausschnitt wieder. 


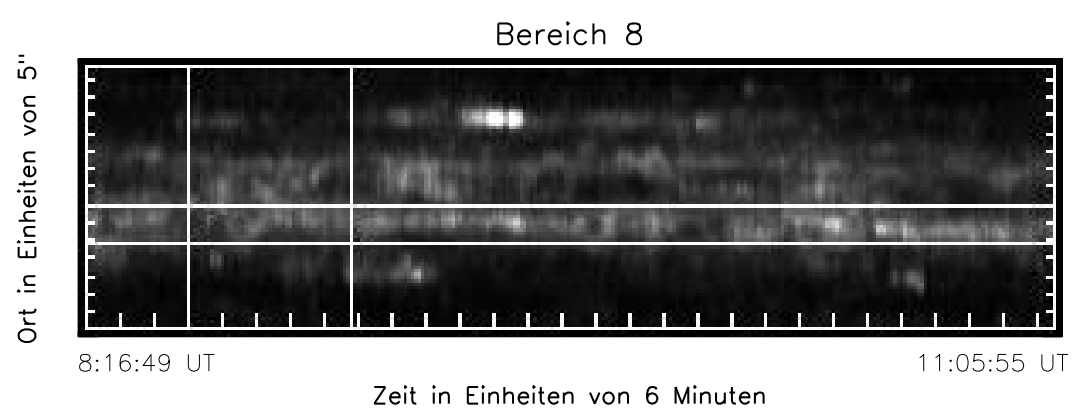

Abbildung 11.58: Bereich Nr. 8 auf dem Hintergrund des C IV-Diagramms.

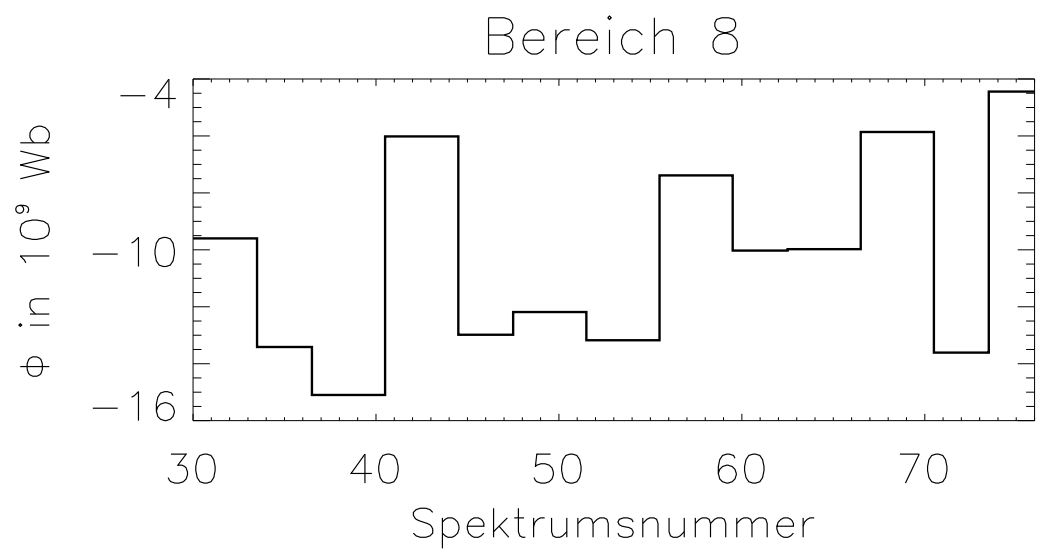

Abbildung 11.59: Über den betrachteten Ortsbereich integrierter magnetischer Fluß.

\subsection{Bereich 8}

Der Bereich 8 (Abb. 11.58) ist mit 9," $5 \times 1692 \mathrm{~s}(28$ min $12 \mathrm{~s})$ der am längsten verfolgte Ausschnitt. Er überdeckt einen Großteil der Entwicklung der negativen Polarität. Der Verlauf des über den entsprechenden Ortsbereich gemittelten magnetischen Flusses ist in Abb. 11.59 dargestellt. Den Abbildungen 11.62/63 und 11.64/65 kann man entnehmen, daß die C IV- wie auch die Ne VIII-Linie, gänzlich anders als im Bereich 7, eine Verbreiterung und Intensitätssteigerung erfahren. Bei den Spektrumsausschnitten der Ne VIII-Linie Abb. 11.65 zeigt sich darüberhinaus eine, wenn auch nur leichte, Blauverschiebung. 


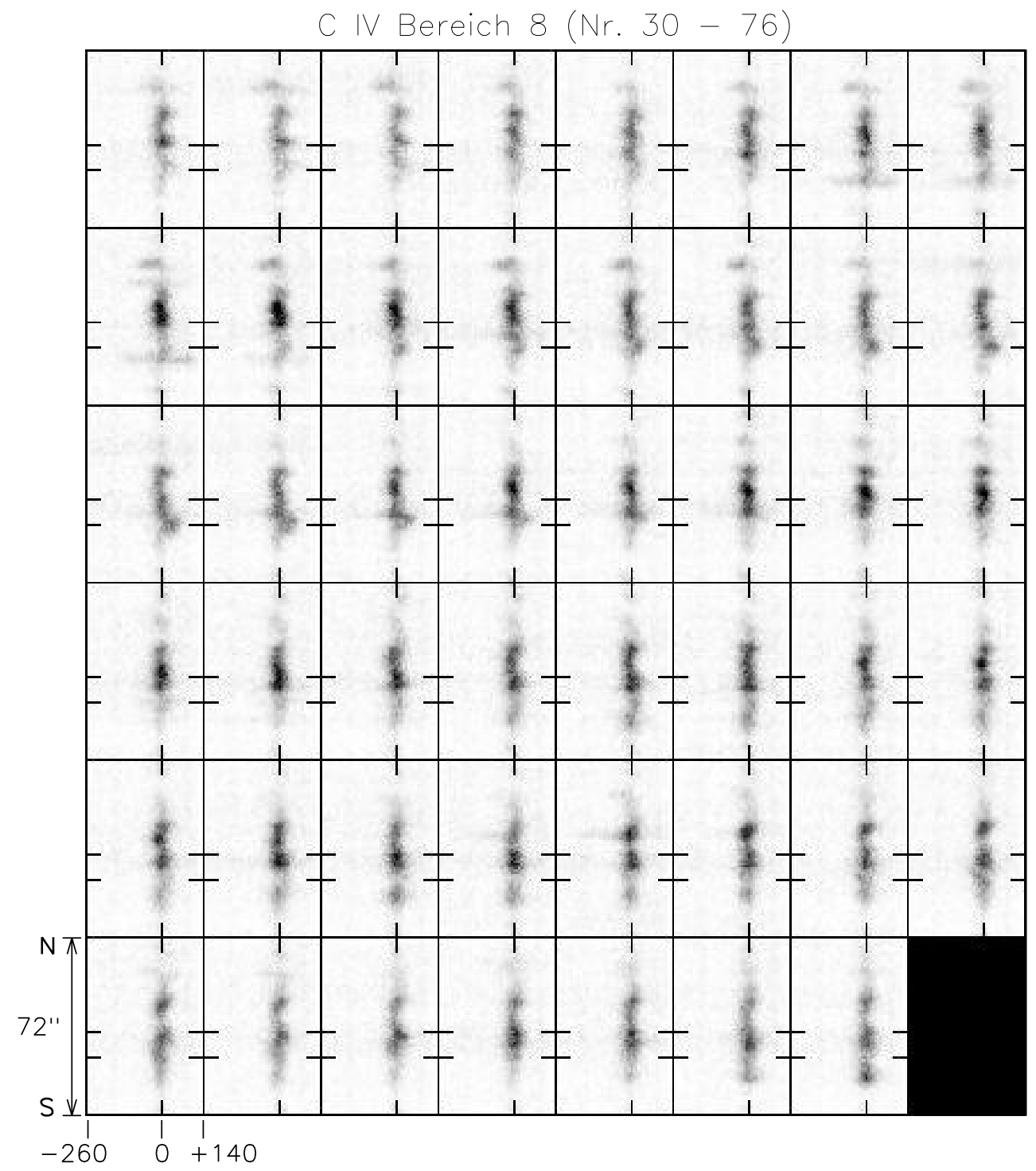

Geschwindigkeit in $\mathrm{km} / \mathrm{s}$

Abbildung 11.60: Spektren der C IV-Linie aus dem Bereich $8(\Delta \lambda \approx 0,21 \mathrm{~nm}, \Delta x \approx$ $\left.72^{\prime \prime}\right)$. Die kleinen vertikalen Striche oben und unten in jedem Spektrum markieren die $\lambda_{0}$-Position. Die jeweils zwei horizontalen Striche links und rechts in jedem Spektrum markieren den betrachteten Ortsbereich. Der zeitliche Abstand zwischen den Spektren beträgt $36 \mathrm{~s}$. 


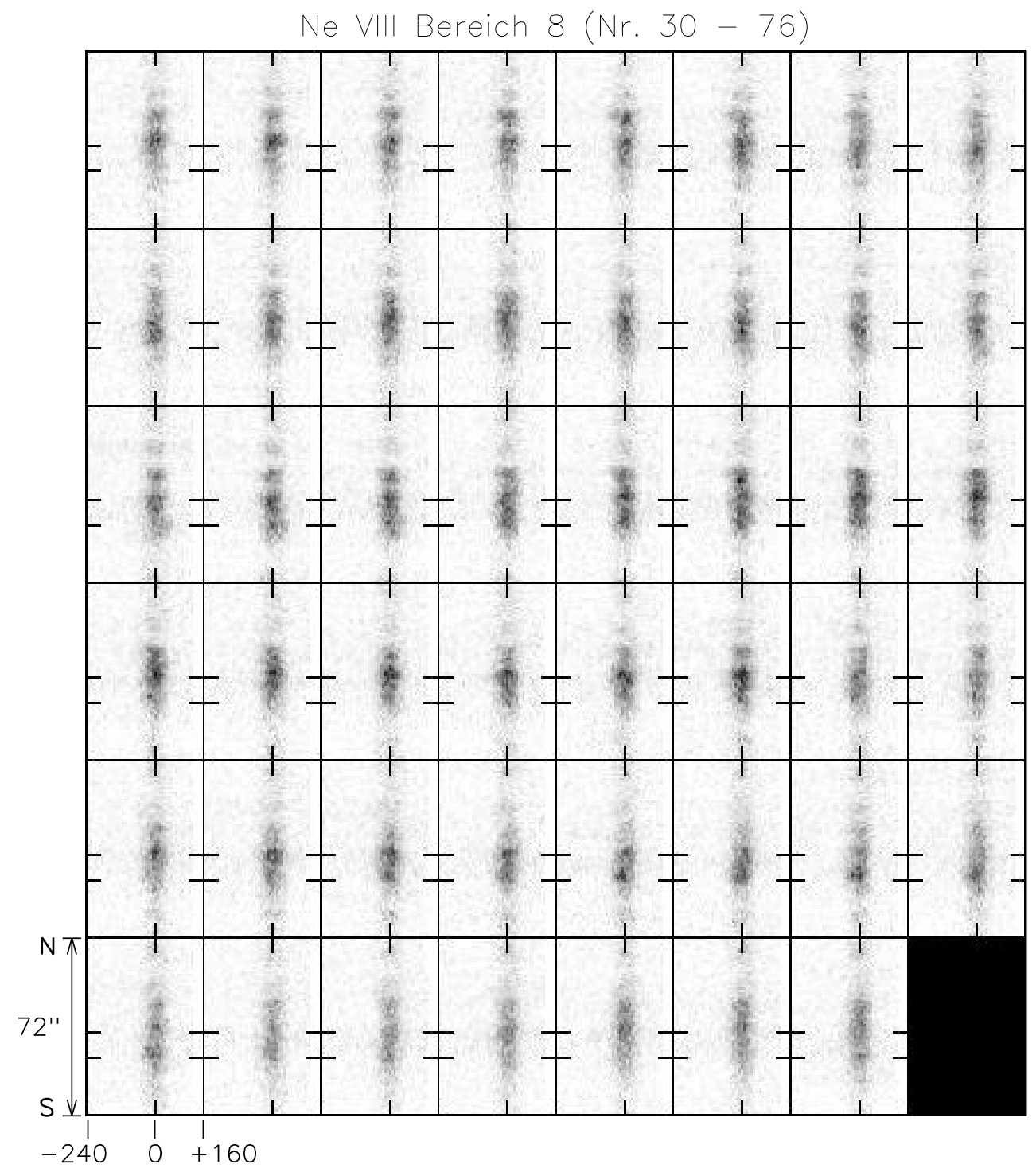

Geschwindigkeit in $\mathrm{km} / \mathrm{s}$

Abbildung 11.61: Spektren der Ne VIII-Linie aus dem Bereich $8(\Delta \lambda \approx 0,21 \mathrm{~nm}, \Delta x \approx$ $\left.72^{\prime \prime}\right)$. Die kleinen vertikalen Striche oben und unten in jedem Spektrum markieren die $\lambda_{0}$-Position. Die jeweils zwei horizontalen Striche links und rechts in jedem Spektrum markieren den betrachteten Ortsbereich. Der zeitliche Abstand zwischen den Spektren beträgt $36 \mathrm{~s}$. 


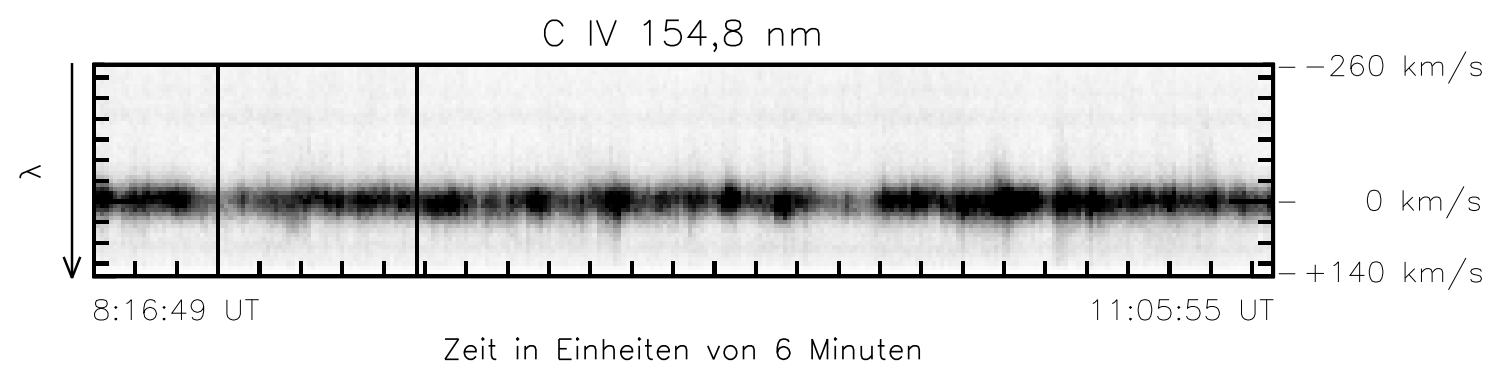

Abbildung 11.62: $\lambda t$-Diagramm C IV-Linie aus dem Bereich 8. Die zwei schwarzen vertikalen Linien grenzen den betrachteten Bereich zeitlich ein. Die Einheit auf der Geschwindigkeitsachse beträgt $\sim 40 \mathrm{~km} / \mathrm{s}$.

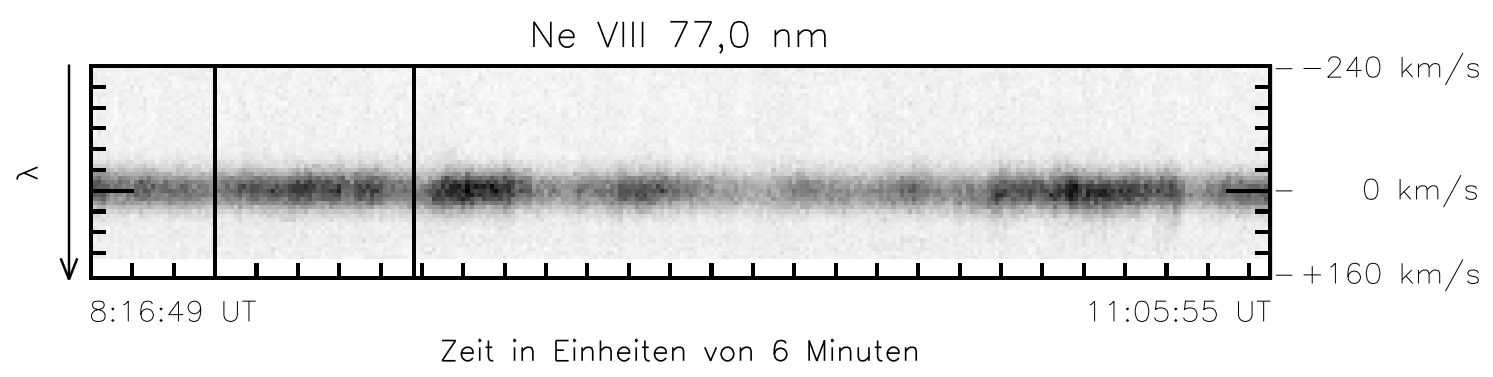

Abbildung 11.63: $\lambda t$-Diagramm Ne VIII-Linie aus dem Bereich 8. Die zwei schwarzen vertikalen Linien grenzen den betrachteten Bereich zeitlich ein. Die Einheit auf der Geschwindigkeitsachse beträgt $\sim 40 \mathrm{~km} / \mathrm{s}$. 

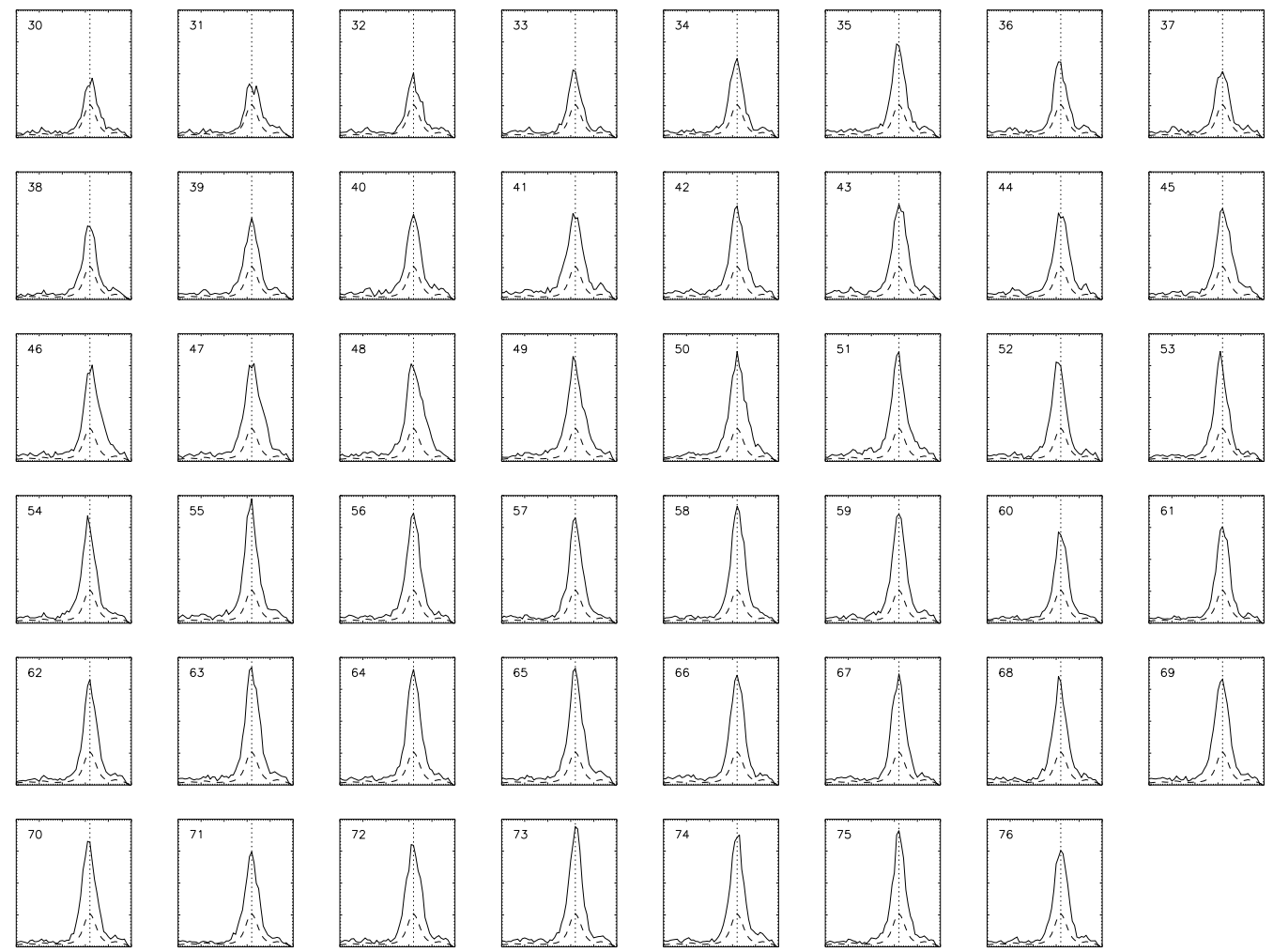

Abbildung 11.64: Über den betrachteten Ortsbereich gemittelte C IV-Spektren aus dem betrachteten Zeitraum. Die Intensität ist in relativen Einheiten gegen die Wellenlänge aufgetragen ( $\lambda$ steigt nach rechts an). Die vertikale, gepunktete Linie markiert die $\lambda_{0}$-Position, und das gestrichelte Profil gibt den gemittelten Spektrumsausschnitt wieder. 

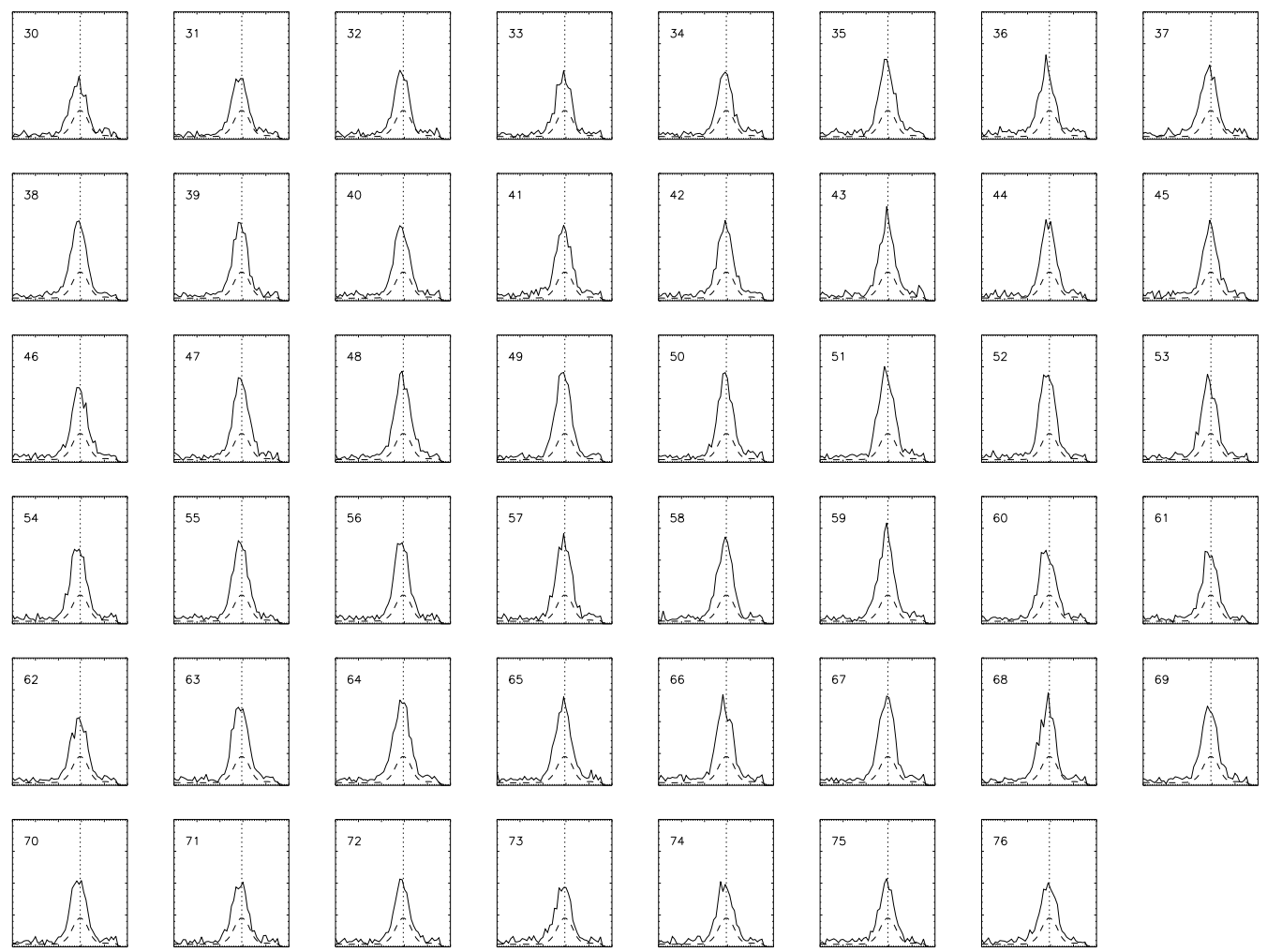

Abbildung 11.65: Über den betrachteten Ortsbereich gemittelte Ne VIII-Spektren aus dem betrachteten Zeitraum. Die Intensität ist in relativen Einheiten gegen die Wellenlänge aufgetragen ( $\lambda$ steigt nach rechts an). Die vertikale, gepunktete Linie markiert die $\lambda_{0}$-Position, und das gestrichelte Profil gibt den gemittelten Spektrumsausschnitt wieder. 


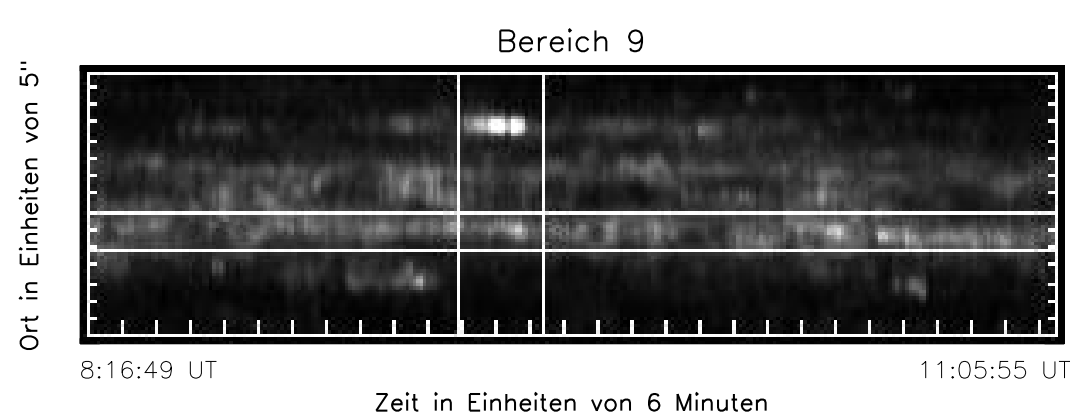

Abbildung 11.66: Bereich Nr. 9 auf dem Hintergrund des C IV-Diagramms.

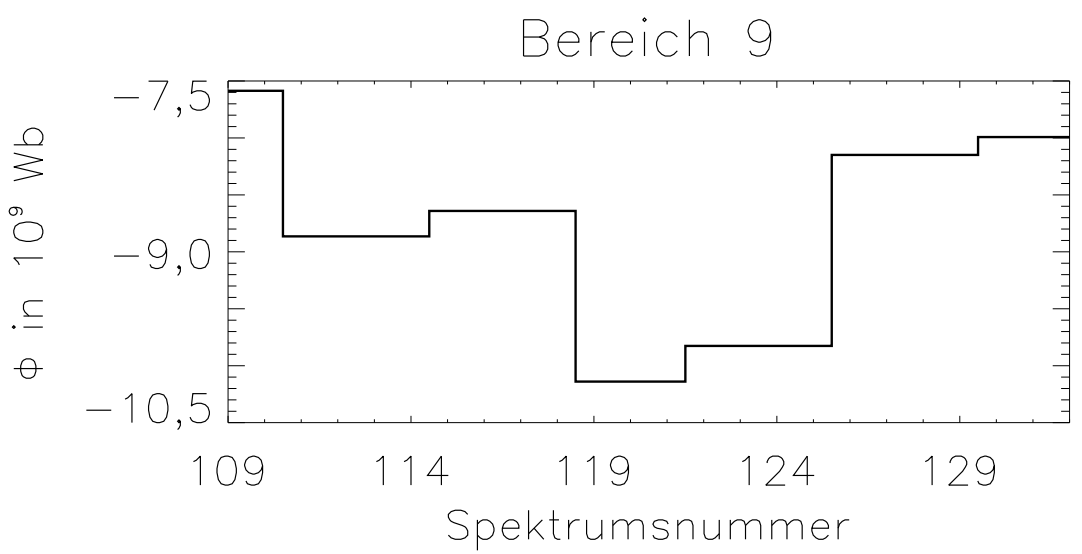

Abbildung 11.67: Über den betrachteten Ortsbereich integrierter magnetischer Fluß.

\subsection{Bereich 9}

Der Bereich 9 hat eine Ausdehnung von 9," $5 \times 864 \mathrm{~s}$ (14 min 24s). Auch er beinhaltet starken magnetischen Fluß negativer Polarität. Die Entwicklung des über den Ortsbereich integrierten Flusses läßt sich aus Abb. 11.67 ablesen. Deutlich zeichnet sich dort ein Extremum zwischen den Spektren Nr. 119 bis $125(\Delta t=252 \mathrm{~s})$ ab. Vergleicht man diese Entwicklung mit der der C IV-Intensität in Abb. 11.68 bzw. 11.72, so stellt man fest, daß auch dort ein Anwachsen und Abfallen stattfindet. Das Maximum dieser Entwicklung liegt bei Spektrum Nr. 126, also um mindestens $36 \mathrm{~s}$ nach hinten verschoben zum Maximum des magnetischen Flusses. Dennoch ist die Gleichartigkeit der zeitlichen Entwicklung gerade im Hinblick auf die im Bereich 7 vorgefundenen Verhältnisse bemerkenswert. Die Ne-Linie zeigt zum Ende des Bereichs hin ein leichtes Anwachsen der Intensität, deren Maximum aber außerhalb des betrachteten Zeitraumes liegt (vgl. Abb. 11.71). 


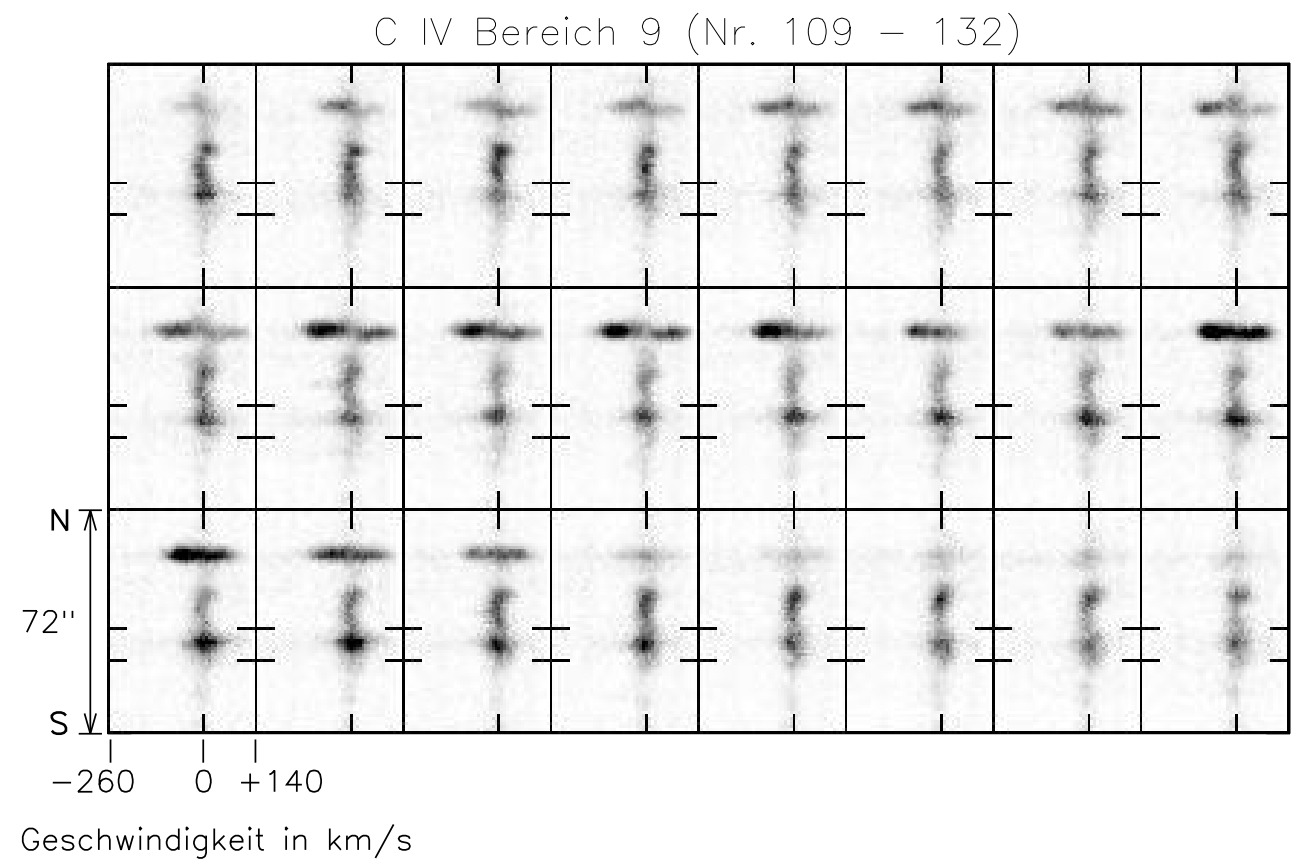

Abbildung 11.68: Spektren der C IV-Linie aus dem Bereich $9(\Delta \lambda \approx 0,21 \mathrm{~nm}, \Delta x \approx$ $\left.72^{\prime \prime}\right)$. Die kleinen vertikalen Striche oben und unten in jedem Spektrum markieren die $\lambda_{0}$-Position. Die jeweils zwei horizontalen Striche links und rechts in jedem Spektrum markieren den betrachteten Ortsbereich. Der zeitliche Abstand zwischen den Spektren beträgt $36 \mathrm{~s}$. 


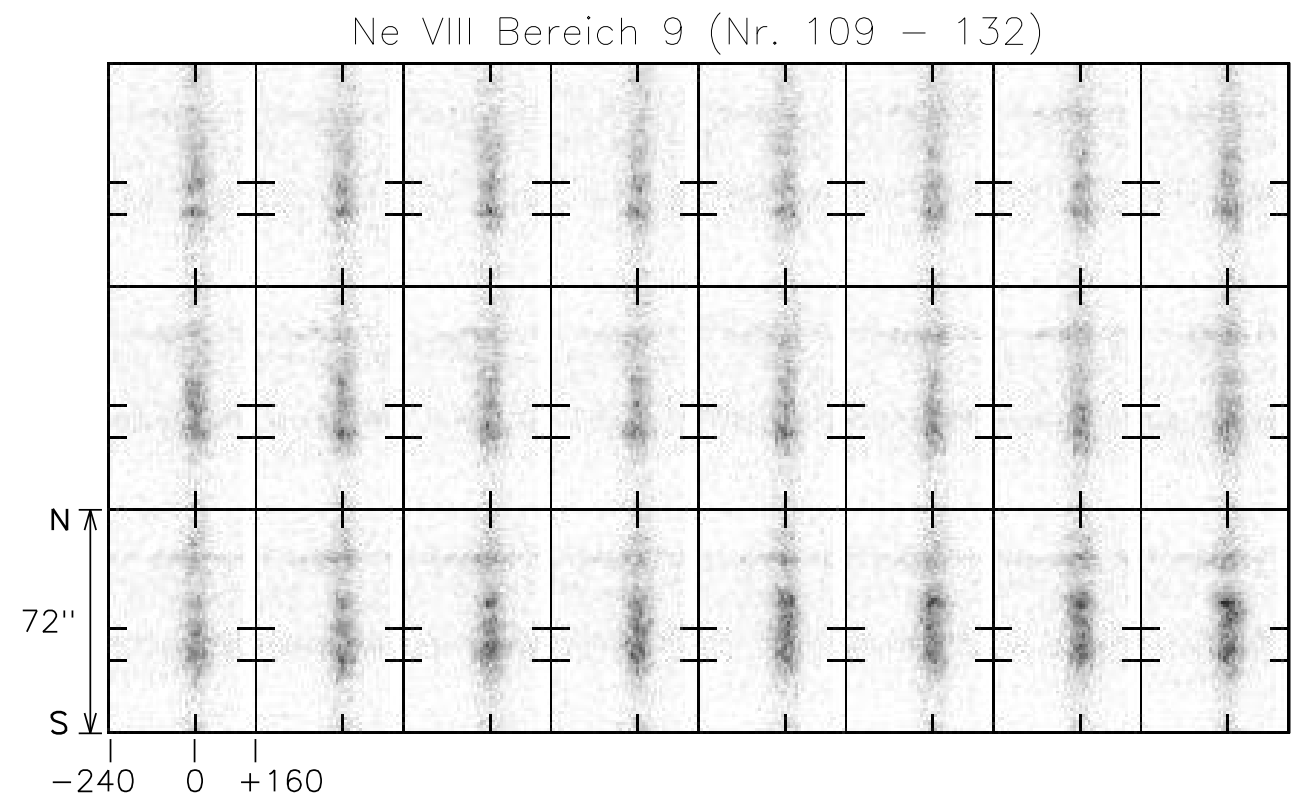

Geschwindigkeit in $\mathrm{km} / \mathrm{s}$

Abbildung 11.69: Spektren der Ne viII-Linie aus dem Bereich $9(\Delta \lambda \approx 0,21 \mathrm{~nm}, \Delta x \approx$ $\left.72^{\prime \prime}\right)$. Die kleinen vertikalen Striche oben und unten in jedem Spektrum markieren die $\lambda_{0}$-Position. Die jeweils zwei horizontalen Striche links und rechts in jedem Spektrum markieren den betrachteten Ortsbereich. Der zeitliche Abstand zwischen den Spektren beträgt 36s.

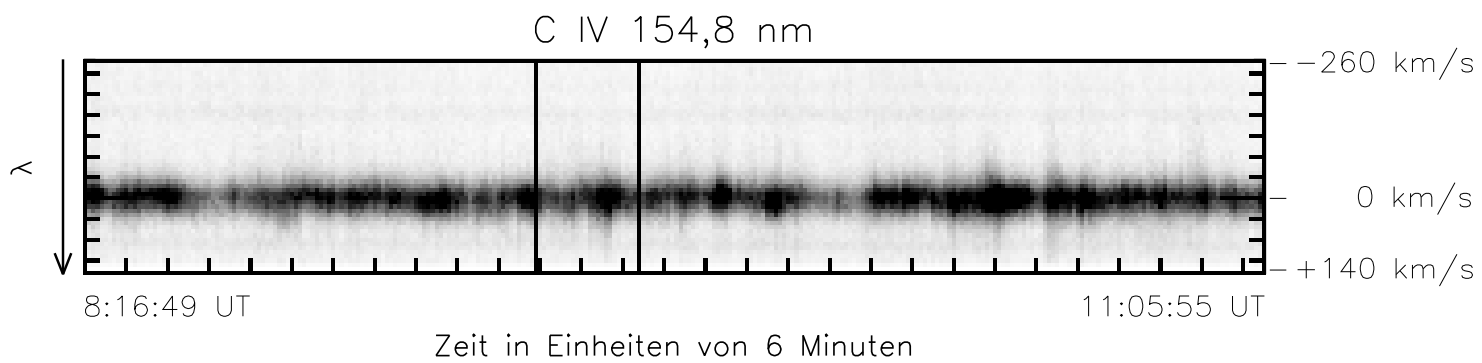

Abbildung 11.70: $\lambda t$-Diagramm C IV-Linie aus dem Bereich 9. Die zwei schwarzen vertikalen Linien grenzen den betrachteten Bereich zeitlich ein. Die Einheit auf der Geschwindigkeitsachse beträgt $\sim 40 \mathrm{~km} / \mathrm{s}$. 


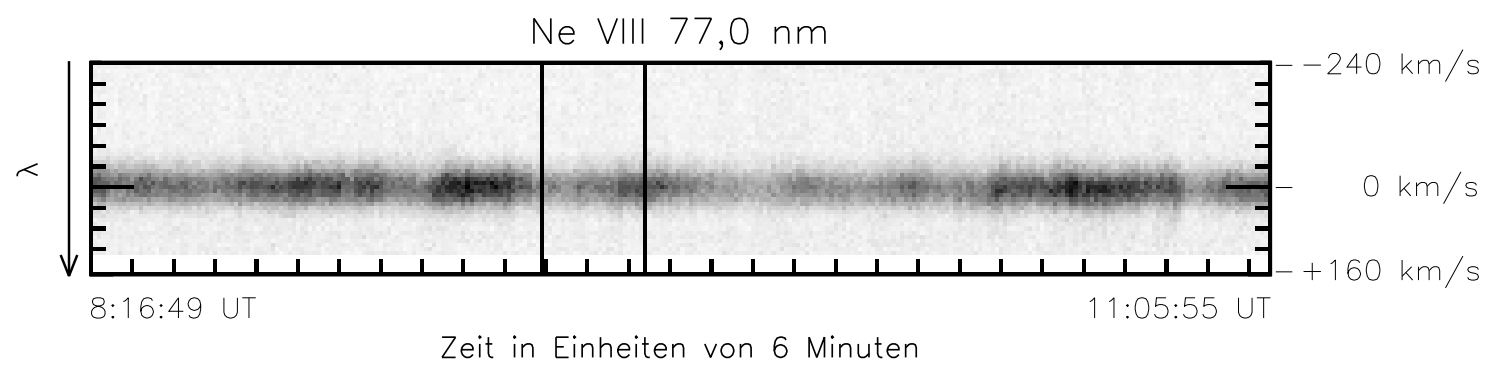

Abbildung 11.71: $\lambda t$-Diagramm Ne viII-Linie aus dem Bereich 9. Die zwei schwarzen vertikalen Linien grenzen den betrachteten Bereich zeitlich ein. Die Einheit auf der Geschwindigkeitsachse beträgt $\sim 40 \mathrm{~km} / \mathrm{s}$.
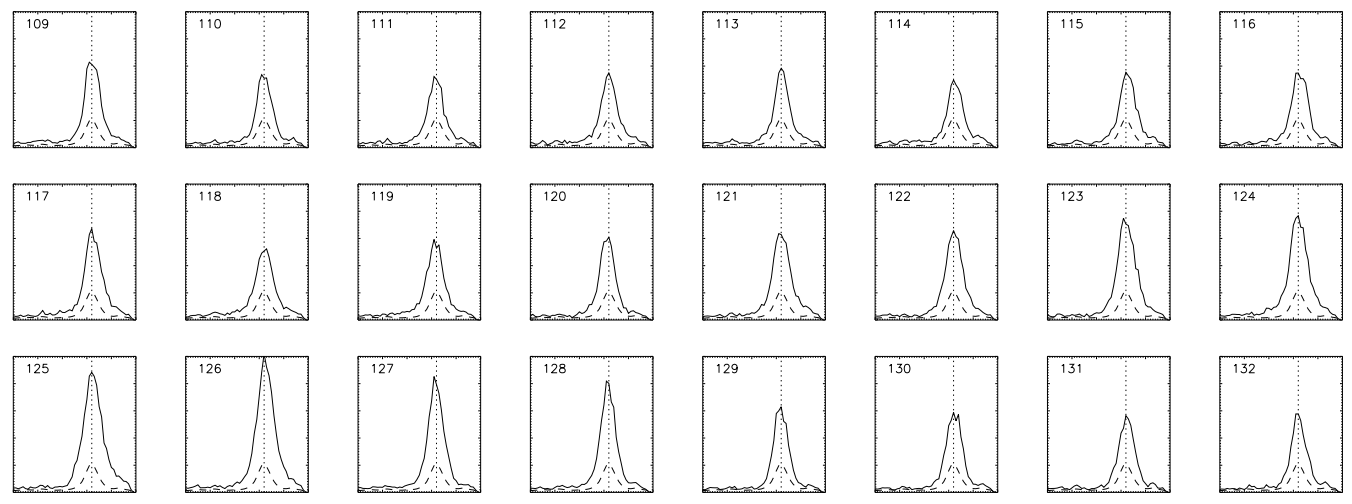

Abbildung 11.72: Über den betrachteten Ortsbereich gemittelte C IV-Spektren aus dem betrachteten Zeitraum. Die Intensität ist in relativen Einheiten gegen die Wellenlänge aufgetragen ( $\lambda$ steigt nach rechts an). Die vertikale, gepunktete Linie markiert die $\lambda_{0}$-Position, und das gestrichelte Profil gibt den gemittelten Spektrumsausschnitt wieder. 

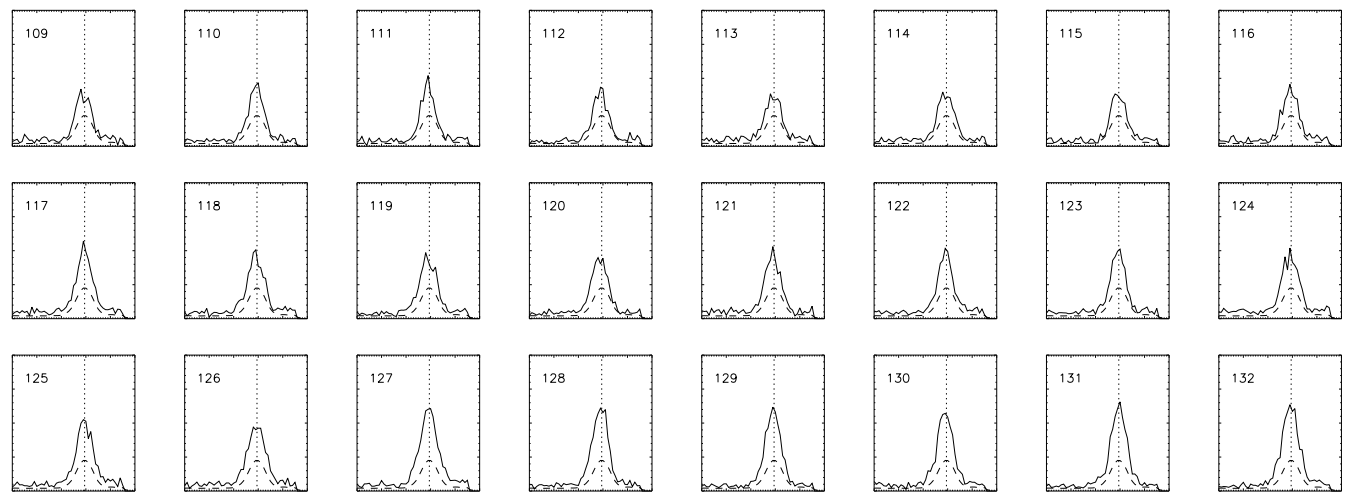

Abbildung 11.73: Über den betrachteten Ortsbereich gemittelte Ne VIII-Spektren aus dem betrachteten Zeitraum. Die Intensität ist in relativen Einheiten gegen die Wellenlänge aufgetragen ( $\lambda$ steigt nach rechts an). Die vertikale, gepunktete Linie markiert die $\lambda_{0}$-Position, und das gestrichelte Profil gibt den gemittelten Spektrumsausschnitt wieder. 


\section{Zusammenfassung}

In dieser Arbeit wurde ein Gebiet mit kleinskaligen Magnetfeldstrukturen aus dem Bereich des chromosphärischen Netzwerkes auf dessen Einfluß auf die darüberliegenden Atmosphärenschichten der Sonne hin untersucht. Dazu wurde ein geeignetes Gebiet in der Sonnenscheibenmitte mit drei Teleskopen (GCT, VTT, SUMER) in verschiedenen Spektralbereichen über ca. 3 Stunde koordiniert beobachtet. Mit Hilfe von zu Beginn der Zeitserien aufgenommenen, großformatigen Übersichtsbildern wurde nicht nur die Anpassung der verschiedenen Datensätze der unterschiedlichen Teleskope untereinander verbessert, sondern auch die beobachtete kleinskalige Struktur in das großräumigere Umfeld eingebettet. Es ist hierbei klar geworden, daß die untersuchten Magnetfelder bzw. Aufhellungen aus dem Bereich des Randes einer Supergranule stammen. Dem mit zweidimensionalen spektropolarimetrischen Methoden ermittelten Datensatz in der Fe I-Linie bei $630,25 \mathrm{~nm}$ wurden Informationen über das photosphärische Magnetfeld entnommen und in Form eines Bildsatzes aufbereitet. Anhand dieses Satzes von Magnetogrammen konnte eine die Beobachtung überlagernde, instrumentelle Bewegung isoliert und kompensiert werden. Die Magnetogramme dienten ferner als Ausgangspunkt für Magnetfeldextrapolationsrechnungen in Potentialfeldnäherung. Weiterhin wurden die Datensätze aus den verschiedenen Spektralbereichen, bedingt durch Einschränkungen bei der Beobachtung mit SUMER, in Form von $x$-Diagrammen miteinander verglichen. Hierbei wurde eine Höhenstaffelung durch geeignete Wahl unterschiedlicher Spektralbereiche für die Untersuchung erreicht. Mit den bodengebundenen Teleskopen GCT und VTT wurden Photosphäre (Fe I-Linie bei 630,25 nm) und Chromosphäre (Ca II $K$-Linie bei 393,3 nm) abgedeckt. Die Erweiterung in die darübergelegenen Schichten gelang durch Beobachtung geeigneter EUV-Linien mit SUMER an Bord der Raumsonde SOHO. Neben einem Kontext-Bild im Licht der He I-Linie bei 58,4 nm wurde die chromosphärische C I-Linie bei 156,10 nm, die der Übergangsregion entstammende C IV-Linie bei 154,82 nm und die koronale Ne VIII-Linie bei 77,04 nm in Form einer rotationskompensierten Zeitserie aufgenommen.

Den Magnetogrammen, Potentialfeldextrapolationen, Dopplergrammen, $x t$-Diagrammen, ergänzt durch gemittelte Profilschnitte aus SUMER-Spektren und damit erzeugte $\lambda t$ Diagramme wurde bezüglich des Untersuchungsobjektes folgendes entnommen:

- Auf dem Rand einer annähernd elliptischen supergranularen Zelle mit einer kleinen Achse von ca. $24^{\prime \prime}(\widehat{\approx} 17000 \mathrm{~km})$ und einer großen Achse von etwa $47^{\prime \prime}(\widehat{\approx} 34000 \mathrm{~km})$ 
sind, sowohl im Lichte des Fe I-Linienzentrums bei $630,25 \mathrm{~nm}$ als auch in der Ca II $K$-Linie bei 393,3 nm, eine ganze Reihe kleiner Aufhellungen $\left(<5^{\prime \prime}\right)$ zu erkennen. Die Aufhellungen sind korreliert mit Gebieten magnetischen Flusses unterschiedlicher Polarität.

- In einem Zeitraum von ca. 4,5 Stunden, in dem etwa alle 133 s ein Magnetogramm von $60^{\prime \prime} \times 57^{\prime \prime}$ entstand, verändern sich die magnetischen Gebiete in kleinräumigen Bereichen rasch, wobei die größere Struktur im wesentlichen erhalten bleibt. Der dabei maximal auftretende magnetische Fluß positiver Polarität beträgt $+7,28$. $10^{9} \mathrm{~Wb}$ (bei einem Auflösungselement von $\left(0,{ }^{\prime \prime} 77\right)^{2}$ entspricht das einer mittleren Flußdichte von $\approx 234 \mathrm{G}=23,4 \mathrm{mT})$. Der magnetische Fluß negativer Polarität erreicht in dieser Zeit ein Minimum von $-4,16 \cdot 10^{9} \mathrm{~Wb}$ (entsprechend einer mittleren Flußdichte von $\approx 134 \mathrm{G}=13,4 \mathrm{mT})$.

- Die Magnetogramme und speziell die Potentialfeldextrapolationen weisen im Zeitraum der ersten etwa 1,5 Stunden (= effektiver gemeinsamer Beobachtungszeitraum) ein Anwachsen und wieder Abfallen der magnetischen Aktivität auf. Die höchsten geschlossenen Magnetfeldlinien erreichen schon in der Potentialfeldextrapolation des ersten Magnetogramms Höhen von über $8000 \mathrm{~km}$.

- Ein an der Position des SUMER-Spaltes (Spaltbreite $=1^{\prime \prime}$ ) aus dem Satz von GCT-Magnetogrammen extrahiertes $x t$-Diagramm weist sowohl ein Gebiet positiver wie auch ein Gebiet negativer Polarität auf. Diese Gebiete verharren über mindestens 1,5 Stunden im wesentlichen am selben Ort, wobei aber der magnetische Fluß bishin zum völligen Verschwinden variiert. Die genannten Variationen erreichen Extrema von $-2,05 \cdot 10^{9} \mathrm{~Wb}$ bis $+3,41 \cdot 10^{9} \mathrm{~Wb}$. Das $x$-Diagramm aus spektral integrierten Spektren der C I-Linie steht, anders als die entsprechenden $x$-Diagramme der C Iv-Linie und Ne vin-Linie, in guter Korrelation zu dem $x t$ Diagramm des magnetischen Flusses. Bemerkenswert ist eine massive Aufhellung in der C IV-Linie im Bereich positiver Polarität. Darüber hinaus zeigen die Diagramme der C IV- und Ne VIII-Linie eine starke räumliche Strukturierung und erhebliche zeitliche Dynamik, die jedoch in keiner Abhängigkeit zum photosphärischen Magnetfeld zu stehen scheint.

- Das $x t$-Dopplergramm aus dem Stokes- $I$-Signal der Fe I-Linie weist Geschwindigkeiten von $-800 \mathrm{~m} / \mathrm{s}$ bis $+700 \mathrm{~m} / \mathrm{s}$ auf. Eine Korrelation mit der zeitlichen Entwicklung des magnetischen Flusses kann hierbei genauso wenig festgestellt werden, wie bei den entsprchenden Diagrammen der C IV- und Ne VIII-Linie. Die Geschwindigkeiten im Falle des C IV- $x$-Dopplergramms reichen von $-70 \mathrm{~km} / \mathrm{s}$ und bis $+40 \mathrm{~km} / \mathrm{s}$, wobei jedoch zumeist die Geschwindigkeiten um $\pm 10 \mathrm{~km} / \mathrm{s}$ liegen. Im Bereich der oben erwähnten Aufhellung findet sich ein Gebiet starker Aufströmung. In dem $x t$-Dopplergramm aus der Ne VIII-Linie finden sich Aufwärtströmungen von bis zu $40 \mathrm{~km} / \mathrm{s}$ und Abwärtsströmungen bis knapp $40 \mathrm{~km} / \mathrm{s}$. Im größten Teil 
des Diagramms pendeln die Werte der Geschwindigkeit zwischen $+5 \mathrm{~km} / \mathrm{s}$ und $-20 \mathrm{~km} / \mathrm{s}$.

- In einem Gebiet, das sowohl in der C IV- als auch in der Ne VIII-Linie eine sich nur geringfügig wandelnde Struktur schwacher Aufhellung aufweist und magnetischen Fluß von nur max. $\pm 6 \cdot 10^{8} \mathrm{~Wb}$ gemischter Polarität umschließt, findet sich in der C IV-Linie eine immer wieder ansteigende und abfallende Blauverschiebung mit relativen Maxima in einem zeitlichen Abstand von $180 \mathrm{~s}, 252 \mathrm{~s}$ und $72 \mathrm{~s}$. Die hierbei erreichten maximalen Geschwindigkeiten betragen etwa $-60 \mathrm{~km} / \mathrm{s}$. Die Ne VIIILinie bleibt hingegen fast völlig in Ruhe.

- In dem Bereich, der das schon erwähnte absolute Helligkeitsmaximum der spektral integrierten C IV-Linie des ganzen Tages umfaßt, findet sich ausschließlich magnetischer Fluß positiver Polarität. Nach Ablauf etwa der Hälfte der Zeit der Aufhellung von ca. 12 min fällt der magnetische Fluß für etwa 2 min von $1,4 \cdot 10^{9} \mathrm{~Wb}$ auf einen Wert kleiner $3 \cdot 10^{8} \mathrm{~Wb}$, während nahezu gleichzeitig die C IV-Aufhellung auf $60 \%$ fällt. Die Profile der C IV-Linie verbreitern sich stark und spalten sich zeitweise in zwei Komponenten auf. Die den entstehenden Rot- und Blauverschiebungen entsprechenden Geschwindigkeiten erreichen dabei Werte von $-145 \mathrm{~km} / \mathrm{s}$ und $+130 \mathrm{~km} / \mathrm{s}$. Es ist festzustellen, daß sich der Ausbruch deutlich flacher aufbaut, als er abfällt. Zeitlich dagegen verschoben zeigt die Ne viı-Linie nur marginale Blauverschiebung. Es ist zu vermuten, daß der Ausbruch in der C IV-Linie eine durch die Integrationszeit von 36 s pro Spektrum verdeckte, nicht aufgelöste Substruktur enthält.

- In einem weiteren Bereich findet eine simultane Blauverschiebung in der C IV- und Ne VIII-Linie statt. Hier wurden maximal $-80 \mathrm{~km} / \mathrm{s}$ für die C IV- und $-110 \mathrm{~km} / \mathrm{s}$ für die Ne VIII-Linie gefunden. Während aber in der Ne VIII-Linie die Blauverschiebung mit sich wandelnder Stärke über ca. 11 min anhält, verändert sich die Verschiebung in der C IV-Linie rasch in eine allgemeine Verbreiterung, die dann schnell schwächer wird und nach etwa 4 min dem mittleren Profil entspricht.

- Nahezu symmetrische Verbreiterung und Aufhellung in der C IV- und Ne VIII-Linie wurde in einem Bereich etwa mittig zwischen den Gebieten unterschiedlicher Polarität gefunden. Anders als in der C IV-Linie, die Aufhellungen in 4 Intervallen über insgesamt $12 \mathrm{~min}$ aufweist, hellt sich die Ne viII-Linie nur einmal für $\sim 4 \mathrm{~min}$ Dauer auf. In einem weiteren Bereich hellen sich beide Linien simultan auf und verbreitern sich symmetrisch.

- In einen Bereich südlich von der negativen Polarität in einem Gebiet, in dem kein signifikanter magnetischer Fluß nachgewiesen werden konnte, fällt ein etwa zweiminütiges Ereignis massiver Rotverschiebung in der C IV-Linie. Die dabei erreichten Geschwindigkeiten konnten vom Detektor nicht vollständig erfaßt werden, lie- 
gen aber mit Sicherheit bei Werten deutlich größer als $+140 \mathrm{~km} / \mathrm{s}$. Die Ne VIII-Linie bleibt während dieses Ereignisses in fast vollständiger Ruhe.

- In dem Bereich des stärksten magnetischen Flusses positiver Polarität des Beobachtungstages kann weder in der C IV- noch in der Ne VIII-Linie eine Auhellung größer als der Tagesschnitt nachgewiesen werden.

- In einem Bereich sich wandelnder relativ starker magnetischer Aktivität negativer Polarität erfahren die Spektren der C IV- wie auch die der Ne VIII-Linie eine Verbreiterung und Intensitätssteigerung, aber keine signifikante Verschiebung.

- In einem anderen Bereich magnetischer Aktivität negativer Polarität wachsen magnetischer Fluß und CIV-Intensität in vergleichbarer Weise. Die Intensität der Ne vIII-Linie wächst, zeitlich deutlich verschoben, auch an. Auch hier findet sich keine signifikante Linienverschiebung.

Es konnte bestätigt werden, daß explosive Ereignisse in Ausbruchsfolgen (engl. bursts) mit individuellen Ereignisdauern von wenigen Minuten auftreten (vgl. Dere 1994, Innes 1997). Das Ereignis größter Rot- und Blauverschiebung liegt in einem Bereich stärkster Aufhellung. Hier wurde auch eine räumliche Verschiebung zwischen rotem und blauem Linienflügel verzeichnet. Weiterhin konnte im Zeitraum des Ausbruchs ein stark variierendes Magnetfeld positiver Polarität nachgewiesen werden. Daß das explosive Ereignis unmittelbare Folge einer magnetischen Rekonnexion durch neuerlich aus dem Sonnenkörper austretenden magnetischen Fluß oder einer magnetischen Auslöschung, bedingt durch die Annäherung von Gebieten unterschiedlicher Polarität, ist, konnte nicht beobachtet werden. Es ist an dieser Stelle hervorzuheben, daß das Auflösungselement $\left(0,{ }^{\prime \prime} 77\right)^{2}$ beträgt und somit die kleinskaligere magnetische Struktur prinzipiell nicht nachweisbar war. Bezeichnend ist aber in diesem Zusammenhang, daß in dem deutlich schwächer fluktuierenden Gebiet negativer Polarität zwar eine Verbreiterung und Intensitätssteigerung der Linienprofile, jedoch im wesentlichen keine Verschiebung beobachtet werden konnte. Dieses Verhalten ist jenem ähnlich, daß in einem Gebiet zwischen den magnetischen Bereichen unterschiedlicher Polarität gefunden wurde. Ferner konnte, wie aus dem Beschriebenen schon hervorgeht, keine einheitliche Charakteristik der Entwicklung der Ereignisse gefunden werden. Auch scheint die Dynamik der Ne VIII-Linie im wesentlichen unabhängig von der der C IV-Linie zu sein. Besonders erwähnenswert ist in diesem Zusammenhang ein Ereignis extrem kurzzeitiger sehr starker Rotverschiebung in der C IV-Linie. Dieses Ereignis von etwa 2 min bildet das Ende einer ca. 30 minütigen Aufhellungsphase, und findet in einem Gebiet statt, in dem kein Magnetfeld nachgewiesen werden konnte. Eine Entsprechung in der Ne VIII-Linie findet sich nicht.

Wie schon erwähnt, unterscheidet sich das Aufhellungsmuster der C IV-Linie stark von dem der Ne viII-Linie. Weiterhin ist festzustellen, daß die Aufhellungen weder der C IVLinie noch der Ne VIII-Linie die magnetische Signatur auf photosphärischem Niveau 
nachzeichnen. Es ist daher sehr schwierig die Ursachen für die Aufhellungen in der Übergangsregion und unteren Korona direkt aus der Dynamik photosphärischer Magnetfelder abzuleiten.

Magnetohydrodynamische Überlegungen lassen in der einige $1000 \mathrm{~km}$ dicken Schicht zwischen Photosphäre und unterer Korona, die durch dramatische Abnahme des Drucks und der Dichte sowie einem massiven Anstieg der Temperatur geprägt ist, relativ komplizierte Magnetfeldkonfigurationen sehr wahrscheinlich erscheinen. So weiten sich z.B. mit abnehmendem Druck magnetische Flußröhren stark auf (Canopy-Effekt). Auch können Flußröhren aufgrund dynamischer Ereignisse stark gekrümmt, verdrillt oder anderweitig verformt sein. Es wären dann diese komplexen und darüber hinaus dynamischen magnetischen Strukturen des solaren Plasmas, die an Orten in höheren Schichten zu Aufhellungen führen, die geometrisch keinerlei Ähnlichkeit zu den Mustern der magnetischen Gebiete auf photosphärischem Niveau haben. 


\section{Literaturverzeichnis}

Bergmann, L., Schaefer, C., 1987: Lehrbuch der Experimentalphysik. III. Optik.

8. Auflage, de Gruyter, Berlin

Bianda, M., Solanki, S. K., Stenflo, J. O., 1998: Hanle depolarisation in the solar chromosphere.

Astronomy and Astrophysics 331, 760

Born, M., Wolf, E., 1959: Principles of Optics.

Pergamon Press, London

Brault, J. W., Neckel, H., 1987: Spectral atlas of Solar absolute disk-averaged and disk-center intensities from 3290 to $12510 \AA$.

Solar Physics 184, 421

Bray, R. J., Loughhead, R. E., Durrant, C. J., 1984: The solar granulation. Cambridge University Press, second edition

Brueckner, G. E., Bartoe, J.-D. F., 1983: Observations of high-energy jets in the corona above the quiet Sun, the heating of the corona, and the acceleration of the solar wind.

Astrophysical Journal 272, 329

Chandrasekhar, S., 1960: Radiative Transfer. Dover

Clark, A. Jr., Johnson, H. K., 1967: Magnetic-Field Accumulation in Supergranules. Solar Physics 2, 433

Curdt, W., Brekke P., Feldman, U., Wilhelm, K., Dwivedi, B. N., Schühle, U.,

Lemaire, P., 2001 (in press): The SUMER Spectral Atlas of Solar-Disc Features. to appear in Astronomy and Astrophysics Supplement

Dere, K. P., 1994: Explosive events, magnetic reconnection, and coronal heating. Advances in Space Research 14, 13

Dere, K. P., Bartoe, J.-D. F., Brueckner, G. E., 1989: Explosive events in the solar transition zone.

Solar Physics 123, 41

Dere, K. P., Bartoe, J.-D. F., Brueckner, G. E., Ewing, J., Lund, P., 1991: Explosive events and magnetic reconnection in the solar atmosphere.

Journal of Geophysical Research 96, 9399 
Feldman, U., Dammasch, I. E., Wilhelm, K., 2000: The morphology of the solar upper atmosphere during the sunspot minimum.

Space Science Reviews 93, 411

Fleck, B., Svestka, Z., 1997: The First Results From SOHO. Kluwer Academic Publishers

Galloway, D. J., Weiss, N. O., 1981: Convection and magnetic fields in stars. Astrophysical Journal 243, 945

Galloway, D. J., Proctor, M. R. E., 1983: The kinematics of hexagonal magnetoconvection.

Geophysical and Astrophysical Fluid Dynamics 24, 109

Gingerich, O., Noyes, R.W., Kalkofen, W., Cuny, Y., 1970: The Harvard-Smithsonian Reference Atmosphere.

Solar Physics 18, 347

Glassmeier, K.-H., Scholer, M.,1991: Plasmaphysik im Sonnensystem. B. I.-Wissenschaftsverlag

Grossmann-Doerth, U., Schüssler, M., Steiner, O., 1998: Convective intensification of solar surface magnetic fields: results of numerical experiments.

Astronomy and Astrophysics 337, 928

Haken, H., Wolf, H. C., 1983: Atom- und Quantenphysik. Springer-Verlag, Berlin

Hansteen, V. H., Betta, R., Carlsson, M., 2000: Rapid intensity and velocity variations in solar transition region lines.

Astronomy and Astrophysics 360, 742

Harra, L. K., Gallagher, P. T., Phillips, K. J. H., 2000: Characteristics of quiet sun cell and network brightenings.

Astronomy and Astrophysics 362, 371

Hassler, D. M., Dammasch, I. E., Lemaire, P., Brekke, P., Curdt, W.,

Mason, H. E., Vial, J.-C., Wilhelm K., 1999: Solar Wind Outflow and the Chromospheric Magnetic Network.

Science 283, 810

Homann, T., 1996: Spektropolarimetrische Untersuchung von Fackeln an den Polen der Sonne.

Diplomarbeit, Universitäts-Sternwarte Göttingen

Innes, D. E., Inhester, B., Axford, W. I., Wilhelm, K., 1997: Bi-directional plasma jets produced by magnetic reconnection on the Sun.

Nature 386, 811

Innes, D. E., Brekke, P., Germerott, D., Wilhelm, K., 1997: Bursts of explosive events in the solar network.

Solar Physics 175, 341

Innes, D. E., Curdt, W., Dwivedi, B. N., Wilhelm, K., 1998: Ultraviolet observations of a dynamic event in the solar corona.

Solar Physics 181, 103 
Innes, D. E., Tóth, G., 1998: Simulations of small-scale explosive events on the sun. Solar Physics 185, 127

Jackson, J. D., 1982: Klassische Elektrodynamik 2. Auflage, de Gruyter

Kippenhahn, R., Möllenhoff, C., 1975: Elementare Plasmaphysik

B. I.-Wissenschaftsverlag

Krieg, J., 1994: Untersuchung der räumlichen und magnetischen Feinstruktur von Umbral Dots mit Methoden der rechnergestützten Daten- und Bildverarbeitung. Diplomarbeit, Universitäts-Sternwarte Göttingen

Krieger, A. S., Timothy, A. F., Roelof, E. C., 1973: A Coronal Hole and Its Identification as the Source of a High Velocity Solar Wind Stream. Solar Physics 29, 505

Krucker, S., Benz A. O., Bastian, T. S., Acton, L. W., 1997: X-ray network flares of the quiet sun. Astrophysical Journal 488, 499

Landi Degl'Innocenti, E., 1992: Magnetic Field Measurements. in: Sánchez, F., Collados, M., Váquez, M., 1992: Solar Observations: Techniques and Interpretation.

Cambridge University Press

Leighton, R. B., Noyes, R. W., Simon, G. W., 1962: Velocity Fields in the Solar Atmosphere. I. Preliminary Report.

Astrophysical Journal 135, 474

Lemaire, P., Wilhelm, K., Curdt, W., Schühle, U., Marsch, E., Poland, A. I., Jordan, S. D., Thomas, R. J., Hassler, D. M., Vial, J.-C., Kühne, M., Huber, M. C. E., Siegmund, O. H. W., Gabriel, A., Timothy, J. G.,

Grewing, M., 1997: First results of the SUMER telescope and spectrometer on SOHO - II. Imagery and Data Management. Solar Physics 170, 105

Lipson, S. G., Lipson, H. S., Tannhauser, D. S., 1997: Optik. Springer-Verlag, Berlin

Lites, B. W., Leka, K. D., Skumanich, A., Martínez Pillet, V., Shimizu, T., 1996: Small-Scale Horizontal Magnetic Fields in the Solar Photosphere. Astrophysical Journal 460, 1019

Livingston, W. C., Harvey, J., 1975: A New Component of Solar Magnetism - The Inner Network Fields.

Bulletin of the American Astronomical Society 7, 346

Lückefedt, E., 1997: Spektropolarimetrische Untersuchung an polaren Fackeln und am extremen Sonnenrand.

Diplomarbeit, Universitäts-Sternwarte Göttingen

Martin, S. F., 1988: The Identification and Interaction of Network, Intranetwork, and Ephemeral Region Magnetic Fields.

Solar Physics 117, 243 
Martin, S. F., Harvey, K. L., 1979: Ephemeral active regions during solar minimum. Solar Physics 64, 93

Mayer-Kuckuk, T., 1985: Atomphysik.

Teubner

Mihalas, D., 1978: Stellar Atmospheres.

W. H. Freeman and Company

Parker, E. N., 1963a: Kinematical Hydromagnetic Theory and its Application to the Low Solar Photosphere.

Astrophysical Journal 138, 552

Pike, C. D., Mason, H. E., 1998: Rotating transition region features observed with the SOHO coronal diagnostic spectrometer.

Solar Physics 182, 333

Porter, J. G., Moore, R. L., Reichmann, E. J., Engvold, O., Harvey, K. L.,

1987: Microflares in the solar magnetic network.

Astrophysical Journal 323, 380

Priest, E. R., 1982: Solar Magnetohydrodynamics.

D. Reidel Publishing Company

Rabin, D., Dowdy, J. F. Jr., 1992: Pervasive variability in the quiet solar transition region.

Astrophysical Journal 398, 665

Rees, D. E., Semel, M. D., 1979: Line Formation in an Unresolved Magnetic Element: A Test of the Centre of Gravity Method.

Astronomy and Astrophysics 74, 1

Scheffler, H., Elsässer, H., 1974: Physik der Sterne und der Sonne.

B. I.-Wissenschaftsverlag

Seehafer, N., 1975: On a Reliable Representation of a Class of Force-Free Solar Magnetic Fields.

Astronomische Nachrichten 296, 177

Seehafer, N., 1978: Determination Of Constant $\alpha$ Force-Free Solar Magnetic Fields From Magnetograph Data.

Solar Physics 58, 215

Seehafer, N., Staude, J., 1979: Force-free Magnetic Field Extrapolation for the Complex Sunspot Group of August 1972.

Astronomische Nachrichten 300, 151

Semel, M. D., 1967: Contribution a L'Etude des Champs Magnétiques dans Régions Actives Solaires.

Annales D'Astrophysique 30, 513

Semel, M. D., 1980: Un analyseur précis de polarisation optique.

Astronomy and Astrophysics 91, 369

Semel, M. D., 1981: Magnetic Fields Observed in a Sunspot and Faculae Using 12 Lines Simultaneously.

Astronomy and Astrophysics 97, 75 
Shurcliff, W. A., 1962: Polarised Light.

Havard University Press

Sigwarth, M., 1999: Dynamik solarer Magnetfelder.

Dissertation, Albert-Ludwigs-Universität Freiburg im Breisgau

Smithson, R. C., 1975: Observations of Weak Solar Magnetic Fields with the Lockheed Diode Array Magnetograph.

Bulletin of the American Astronomical Society 7, 346

Solanki, S. K., Finsterle, W., Rüedi, I., Livingston, W., 1999: Expansion of solar magnetic flux tubes large and small.

Astronomy and Astrophysics 347, 27

Steiner, O., Grossmann-Doerth, U., Knölker, M., Schüssler, M., 1998: Dynamical Interaction of Solar Magnetic Elements and Granular Convection: Results of a Numerical Simulation.

Astrophysical Journal 495, 468

Stenflo, J. O., 1982: The Hanle Effect and the Diagnostics of Turbulent Magnetic Fields in the Solar Atmosphere.

Solar Physics 80, 209

Stenflo, J. O., 1994: Solar Magnetic Fields. Kluwer Academic Publishers

Stix, M., 1989: The Sun - An Introduction. Springer-Verlag, Berlin

Stolpe, F., 1994: Untersuchung der räumlichen und magnetischen Feinstruktur solarer Fackelgebiete mit Methoden der rechnergestützten Daten- und Bildverarbeitung. Diplomarbeit, Universitäts-Sternwarte Göttingen

Stolpe, F., 1998: Magnetfelder mit geringem magnetischen Fluß in der Sonnenatmosphäre. Dissertation, Universitäts-Sternwarte Göttingen

Stolpe, F., Kneer, F., 1998: MISC, an instrument for multi-dimensional spectroscopy.

Astronomy and Astrophysics Supplement Series 131, 181

Stolpe, F., Koschinsky, M., Kneer, F., 1997: 2D-Spectroscopy with the Image Scanner of the Gregory-Coudé-Telescope on Tenerife. Experimental Astronomy 7, 301

Title, A. M., Tarbell, T. D., Topka, K. P., 1987: On the Relation between Magnetic Field Structures and Granulation.

Astrophysical Journal 317, 892

del Toro Iniesta, J.-C., Semel, M. D., Collados, M., Sánchez Almeida, J., 1990: Spectropolarimetry of solar faculae: high spatial resolution results.

Astronomy and Astrophysics 227, 591

Unsöld, A., 1955: Physik der Sternatmospären.

Springer-Verlag, Berlin 
Unsöld, A., Baschek, B., 1994: Der neue Kosmos. Springer-Verlag, Berlin

Vernazza, J. E., Avrett, E. H., Loeser R., 1980: Structure of the solar chromosphere.III. Models of the EUV brightness components of the quiet sun. The Astrophysical Journal Supplement Series 45, 635

Voigt, H.-H., 1980: Abriß der Astronomie.

B. I.-Wissenschaftsverlag

Wang, H., 1988: Structure of Magnetic Fields on the quiet Sun. Solar Physics 116, 1

Wang, J., Wang, H., Tang, F., Lee, J. W., Zirin, H., 1995: Flux Distribution of Intranetwork Magnetic Fields.

Solar Physics 160, 277

Wang, H., Tang, F., Zirin, H., Wang, J., 1996: The Velocities of the Intranetwork and Network Magnetic Fields.

Solar Physics 165, 223

Weiss, N. O., 1964: Magnetic Flux Tubes and Convection in the Sun Monthly Notices of the Royal Astronomical Society 128, 225

Wilhelm, K., Curdt, W., Marsch, E., Schühle, U., Lemaire, P., Gabriel, A.,

Vial, J.-C., Grewing, M., Huber, M. C. E., Jordan, S. D., Poland, A. I.,

Thomas, R. J., Kühne, M., Timothy, J. G., Hassler, D. M.,

Siegmund, O. H. W., 1995: SUMER - Solar Ultraviolet Measurements of Emitted Radiation.

Solar Physics 162, 189

Wilhelm, K., Lemaire, P., Curdt, W., Schühle, U., Marsch, E., Poland, A. I., Jordan, S. D., Thomas, R. J., Hassler, D. M., Huber, M. C. E., Vial, J.-C., Kühne, M., Siegmund, O. H. W., Gabriel, A., Timothy, J. G., Grewing, M.,

Feldman, U., Hollandt, J., Brekke, P., 1997: First results of the SUMER telescope and spectrometer on SOHO - I. Spectra and Spectroradiometry. Solar Physics 170, 75

Zirker, J. B., ed., 1977: Coronal Holes and High Speed Wind Streams. Colorado Associated University Press, Boulder. 


\section{Dank}

Mein Dank gilt Prof. Dr. F. Kneer, der mir die Möglichkeit bot, an der Sternwarte in Göttingen zu promovieren. Ich danke ihm für wertvolle Hinweise und Diskussionen. Weiterhin danke ich der SUMER-Gruppe des Max-Planck-Instituts für Aeronomie in Lindau, im speziellen Dr. D. Gigas und Dr. W. Curdt, für die fruchtbare Zusammenarbeit bei der koordinierten Beobachtung und Datenverarbeitung. Im Rahmen der koordinierten Beobachtung gilt mein Dank außerdem Dr. M. v. Uexküll vom Kiepenheuer-Institut für Sonnenphysik in Freiburg, die mich bei Datenerhebung und -verarbeitung von Seiten des VTTs unterstützte. Desweiteren danke ich Dr. F. Stolpe, dessen Arbeit im Zuge seiner Dissertation Grundlage für die Beobachtungen vom GCT aus waren. Mein Dank gilt darüberhinaus Dr. A. Hofmann vom Astrophysikalischen Institut Potsdam, der mir das Extrapolationsprogramm zur Verfügung stellte und für dessen Anwendung wichtige Hinweise gab. Für wertvolle Anregungen und Diskussionen sei an dieser Stelle C. Ritter, Dr. J. Hirzberger, Dr. M. Koschinsky, Dr. A. Wittmann, Dr. E. Wiehr, K. Janssen, I. Berentzen, Dr. J. Krieg und im speziellen für die Rechnerbetreuung Dr. B. Jucknischke, Dr. P. Sütterlin, M. Wunnenberg und Dr. K. Reinsch gedankt. Eine finanzielle Förderung dieser Arbeit wurde im Rahmen des Projekts "Kleinskalige Magnetfelder der Sonne und ihr Einfluß auf Chromosphäre, Übergangszone und Korona" von der Deutschen Forschungsgemeinschaft (Aktenzeichen KN 152/20-1,2,3) übernommen. 


\section{Lebenslauf}

Name: $\quad$ Volker Wilken

Geburtsdatum: $\quad$ 01. November 1968

Geburtsort: Northeim

Staatsangehörigkeit: deutsch

Schulbildung: $\quad$ 1975-1979 Martin-Luther-Grundschule Northeim

1979-1981 Thomas-Mann-Schule, Orientierungsstufe

Northeim

1981-1985 Gymnasium-Corvinianum Northeim

1985-1988 Technisches Gymnasium Einbeck

Mai 1988 Abitur

Wehrdienst: $\quad$ Oktober 1988 - Dezember 1989

Studium: Georg-August-Universität Göttingen

immatrikuliert seit WS 1989/90

Diplomstudiengang Physik

April 1992 Vordiplom

Diplomarbeit an der Universitäts-Sternwarte mit dem Titel:

"Untersuchung der Mitte-Rand-Variation der solaren

Granulation mit Methoden der Speckle-Interferometrie".

Mai 1996 Diplom

Tätigkeiten: Juni 1996 - Oktober 1996 wissenschaftliche Hilfskraft an der Universitäts-Sternwarte Göttingen.

Anfertigung einer Veröffentlichung mit dem Titel :

"Speckle measurements of the centre-to-limb

variation of the solar granulation".

November 1996 Beginn der Dissertation an der

Universitäts-Sternwarte Göttingen mit dem Titel :

"Kleinskalige Magnetfelder der Sonne und ihr Einfluß auf Chromosphäre, Übergangszone und Korona ". 